Tianle Cheng

Michigan Technological University

Follow this and additional works at: https://digitalcommons.mtu.edu/etds

Part of the Materials Science and Engineering Commons

Copyright 2012 Tianle Cheng

Recommended Citation

Cheng, Tianle, "DIFFUSE-INTERFACE FIELD APPROACH TO MODELING SELF-ASSEMBLY OF

HETEROGENEOUS COLLOIDAL SYSTEMS AND RELATED DIPOLE-DIPOLE INTERACTION PHENOMENA", Dissertation, Michigan Technological University, 2012.

https://doi.org/10.37099/mtu.dc.etds/620

Follow this and additional works at: https://digitalcommons.mtu.edu/etds

Part of the Materials Science and Engineering Commons 


\title{
DIFFUSE-INTERFACE FIELD APPROACH TO MODELING SELF-ASSEMBLY OF HETEROGENEOUS COLLOIDAL SYSTEMS AND RELATED DIPOLE-DIPOLE INTERACTION PHENOMENA
}

By

TIANLE CHENG

\begin{abstract}
A DISSERTATION
Submitted in partial fulfillment of the requirements for the degree of

DOCTOR OF PHILOSOPHY
\end{abstract}

(Materials Science and Engineering)

MICHIGAN TECHNOLOGICAL UNIVERSITY

2012

๑ 2012 TIANLE CHENG 
This dissertation, "Diffuse-Interface Field Approach to Modeling SelfAssembly of Heterogeneous Colloidal Systems and Related Dipole-Dipole Interaction Phenomena" is hereby approved in partial fulfillment of the requirements for the Degree of DOCTOR OF PHILOSOPHY IN MATERIALS SCIENCE AND ENGINEERING.

Department of Materials Science and Engineering

Signatures:

Dissertation Adviser

Dr. Yu U. Wang

Committee member

Dr. Jaroslaw W. Drelich

Committee member

Dr. Miguel Levy

Committee member

Dr. Dennis Desheng Meng

Department Chair

Dr. Mark R. Plichta

Date 


\section{Tomy wife.}




\section{Table of Contents}

List of figures......................................................... vi

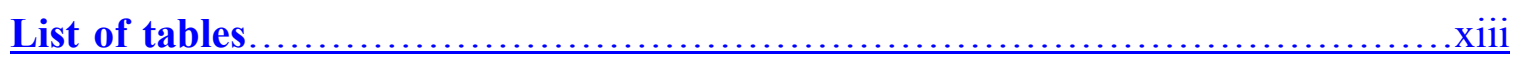

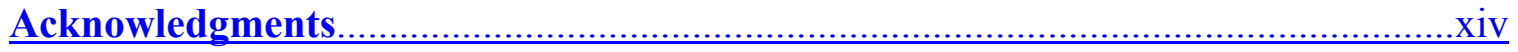

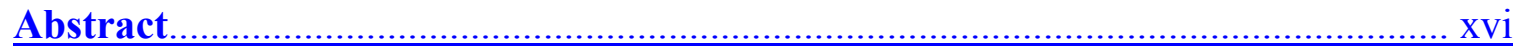

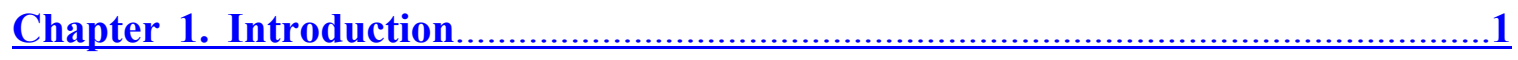

1.1 Colloid as building block for advanced materials......................

1.2 Interparticle forces in colloidal system............................

1.3 Numerical modeling methods on colloid self-assembly: A survey..........8

$1.4 \quad$ DIFA to modeling self-assembly of heterogeneous colloidal particles and dipole-dipole interaction related ferroic domain evolution ...............11

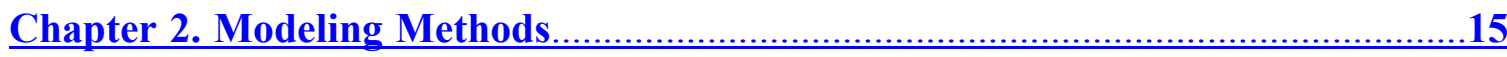

2.1 DIFA to modeling colloidal particles in multiphase liquid colloid system: the Laplace pressure........................................................... 15

2.1.1 DIFA formulation of free energy ................................... 15

2.1.2 Laplace pressure.............................................................16

2.2 Steric interaction, electrostatic/magnetostatic interaction and Brownian force............................................

2.3 Kinetic equations of the multiphase-liquid colloid systems..............25

2.3.1 Kinetic description of particles ..........................25 
2.3.2 Kinetic description of liquid phase separation.................26

2.3.3 Model verification and capability test........................28

2.4 Phase field model for dipolar domains in ferroic materials...............32

2.4.1 Ferroelectric system.......................................32

2.4.2 Ferromagnetic system....................................33

Chapter 3. Colloids at liquid interfaces: The role of Laplace pressure ...........35

3.1 Isolated particles at liquid interfaces............................35

3.2 Colloid jamming at liquid interfaces........................ 41

3.3 Mechanical stability and collapse of monolayer Pickering emulsions....44

3.4 Stability of capillary bridges through Communicating Vessels Effect...50

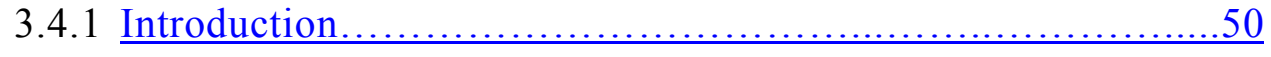

3.4.2 Computational details..................................52

3.4.3 Results and discussion.....................................53

3.5 Additional discussion about lateral capillary interaction...................68

3.6 Supplementary material............................................. 70

3.6.1 Calculation details of capillary bridges.............................70

3.6.2 Calculation details of particles jamming at liquid interfaces........73

Chapter 4. Self-assembly of colloids with heterogeneous charges and dipoles .....77

4.1 Aggregation of oppositely charged particles..............................................77

iv 
4.2 Aggregation of patchy particles.......................................................79

4.3 Formation of ionic colloidal crystal..............................................................

4.4 Dipolar colloids self-assembly under external field control: Magnetic composites................................................94

\section{Chapter 5. DIFA to modeling dipole-dipole interaction related phenomena .......103}

5.1 Internal dipole field and ferroelectric domain stabilization...........................103

5.1.1 Phase field simulation on ferroelectric domain stabilization........103

5.1.2 Charge compensation and aging mechanisms in iron-doped ferroelectric titanate perovskites..................................109

5.2 Magnetic dipole-dipole interaction and magnetization processes in $\mathrm{FePt}$ polytwin crystals under external field .............................116

5.2 .1 Introduction .............................................116

5.2.2 Micromagnetic modeling details ..........................118

5.2.3 Simulation results and discussion..........................121

Chapter 6. Conclusion and discussion ..................................131

6.1 Summary about the DIFA model on colloids system...................131

6.2 Insights from modeling and computer simulations...................132

6.3 Further discussion ................................................134

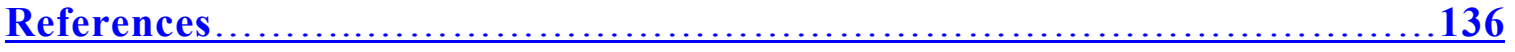

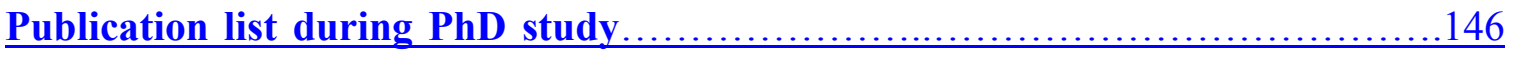




\section{LIST OF FIGURES}

2.1 Schematic of Laplace pressure and interfacial tension imposed on a particle adsorbed at liquid-liquid interface...............................................................18

2.2 Comparison of Laplace pressure across a curved liquid interface as determined from Gibbs-Duhem relation with that determined from Young-Laplace equation.

2.3 Pressure change along a path $\overrightarrow{S E}$ across a curved liquid interface from outside a droplet to inside..

2.4 (a) Schematic of steric repulsion between two particles. (b) Two order parameters (field variables) $\eta$ and $\tilde{\eta}$ are used to describe the "core" and "halo" of one coated particle of pentagon shape. (c) 3D visualization (height representing the value) of the order parameter distributions of $\eta$ and $\tilde{\eta}$

2.5 Simulated jamming of ellipse-shaped particles at liquid interfaces during late stage of spinodal decomposition.

2.6 Surface pressure distribution during the late stages of spinodal decomposition. (a) is corresponding to Figure $2.5 \mathrm{~d}$, and (b) to Figure $2.5 \mathrm{e}$.

3.1 Relaxation of various shaped particles at the surface of a vesicle. (The "halo" fields of particles are not shown.) (a) Ellipse (b) Pentagon (c) Star 39 
3.2 Star shaped particle may choose to relax at a metastable state instead of the energy minimum state, due to the flexibility for contact angle at the vertices.....40

3.3 Diffusional equilibrium between a Pickering emulsion droplet and a free droplet.

3.4 Schematic of liquid interface morphology of an emulsion droplet adsorbed with particles.

3.5 Simulation results show interparticle force of a colloidal shell as a function of Laplace pressure.

3.6 Simulation results show that negative Laplace pressure leads to buckling and collapse of colloidal shell (spherical particles). 45

3.7 Simulation results show that negative Laplace pressure leads to buckling and collapse of colloidal shell (shape-anisotropic particles of different shapes)......46

3.8 Simulation results show that positive Laplace pressure leads to spheroidization of the colloidal shell.

3.9 Simulation results show that colloidal shells exhibit different stable morphology with arbitrary curvature under zero Laplace pressure

3.10 Simulation result shows that attraction between particles allows a Pickering emulsion droplet coexist with a smaller free droplet. .48

3.11 Enlargement of the particle/liquid morphology in highlight boxes of Figure 2.5(d) and (f) .48 
3.12 Simulated nucleation and growth of capillary bridges formed in colloidal crystal during liquid phase separation process.

3.13 (a) Schematic of a capillary bridge between two solid spheres. (b) The Laplace pressure (negative) inside a capillary bridge is a monotonically increasing function of capillary bridge volume $V^{c b}$. (c) Analogy between Laplace pressure stabilizing meniscus size through inter-liquid diffusion (bottom) and hydrostatic pressure equilibrating liquid level through flow in communicating vessels

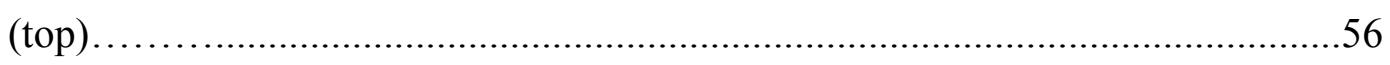

3.14 Contour plot of chemical potential $\mu_{1}=\delta F / \delta c_{1}$ distributed inside the capillary bridges in the highlighted box shown in Figure 3.12 at time step 50,000 .59

3.15 Simulated capillary bridge formation during liquid phase separation in imperfect colloidal crystal.

3.16 Comparison of simulated free energies of different liquid phase distributions in stable and metastable states. .64

3.17 Dependence of interfacial energy associated with a capillary bridge on the capillary bridge volume. .65

3.18 Simulated capillary bridge formation and self-stabilization in saturn-ring-like colloidal superstructure. .67

3.19 Simulated capillary bridge formation and self-stabilization in an interstitial colloidal crystal .67

3.20 Simulated motion of two particles subject to external force (such as gravity) at the surface of a sessile drop. .69 
3.21 Definition of geometrical parameters for a meniscus in a slit between two

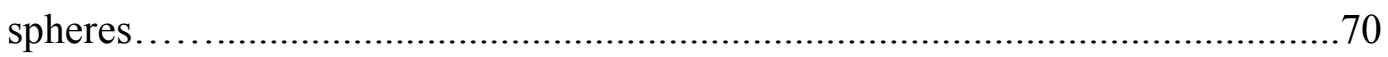

3.22 For acute contact angles, Laplace pressure still monotonically increases with increasing volume of a capillary bridge for small nonzero gap distance $(D=0.02 R)$

3.23 Dependence of filling angle $\beta$ on capillary bridge volume at different contact angles .72

3.24 The Laplace pressure of a capillary bridge (2D) is also a monotonically increasing function of the capillary bridge volume, $V^{c b}$

3.25 Definition of geometrical parameters for a particles jamming at liquid-liquid interfaces.

3.26 Definition of geometrical parameters for a particles jamming at liquid-liquid interfaces. Red solid lines stand for liquid-liquid interfaces. The curvature is negative.

4.1 Simulation results show self-assembly of oppositely charged particles with equal number of particles but unequal charge density.

4.2 Simulation results show self-assembly of oppositely charged particles. .80

4.3 Schematic of two particles with patchy charges 81

4.4 Distribution of electrostatic force between two patchy particles with heterocharges (two-dimensional case) 82 
4.5 Distribution of electrostatic force between two patchy particles with hetero-

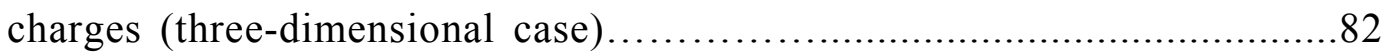

4.6 Simulation result (2D) shows aggregation of patchy particles under electrostatic interaction and Brownian motion, the particles form coexisting dimers finally...84

4.7 Schematic of electrostatic energy profile between two particles with patchy

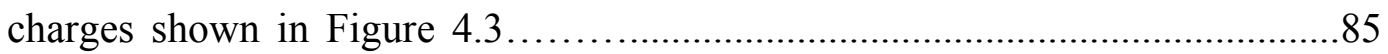

4.8 Simulation result (2D) shows aggregation of patchy particles under electrostatic interaction and Brownian motion, the particles form coexisting trimers, dimers and monomers finally.

4.9 Simulation result (2D) shows aggregation of patchy particles under electrostatic interaction and Brownian motion. There are only monomers finally, i.e., no stable dimers or trimers survive

4.10 Self-assembly of colloidal particles with opposite charges under gravity......92

4.11 Schematic of electric/magnetic field around a dipolar particle .94

4.12 Elliptical colloidal particles with magnetic dipole alignment under constant external field $H^{e x} / M_{s}=0.3$

4.13 Elliptical colloidal particles with magnetic dipole alignment under constant external field, corresponding to Figure 4.12 .98

4.14 Spherical colloidal particles with magnetic dipole alignment under constant external field .98 
4.15 Magnetic field distribution $H / H^{e x}$ inside aligned particles.

4.16 Elliptical colloidal particles with magnetic dipole alignment under increasing external field.

4.17 Real-time composite performance (susceptibility coefficients) evolution, corresponding to Figure 4.16 .

4.18 Comparison of composite performance in four cases: elliptical particles, spherical particles, coated spherical particles under increasing external field and elliptical particles self-assembly without external field applied.

5.1 Computer simulation of domain stabilization and recovery behaviors in sufficiently aged ferroelectrics

5.2 Computer simulation of domain evolution in insufficiently aged ferroelectrics. 106

5.3 Computer simulation of domain evolution in intermediately aged ferroelectrics.

5.4 Schematic: Imperfect perovskite crystal $\mathrm{ABO}_{3}$ taken as superposition of imaginary background perfect lattice (IBL) and Effective charges (ECs).........114

5.5 (a) Perpendicular two shoulder dipoles (b) Parallel two shoulder dipoles......116

5.6 Initial magnetic domain configuration, crystallographic orientation, and applied field directions. 
5.7 Simulated magnetization curves and corresponding magnetic domain structures during magnetization processes under external magnetic fields along the five different directions shown in Figure 5.6.........................................................123

5.8 Bending of magnetic domain wall segments in the configurations B1, C1, and D1,

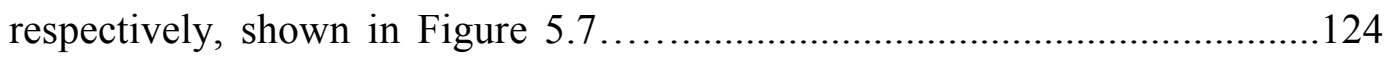

5.9 Close-up view of magnetic charge density and internal magnetic field distributions in the highlighted rectangular region of the domain configuration $\mathrm{C} 1$

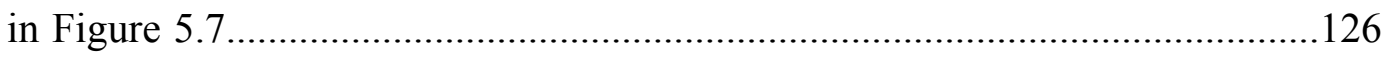

5.10 Close-up view of the highlighted rectangular region in the domain configuration

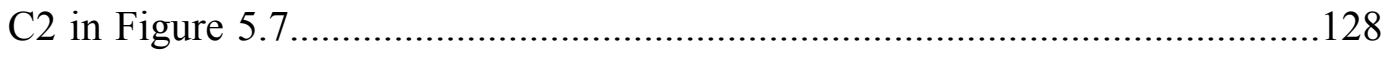




\section{LIST OF TABLES}

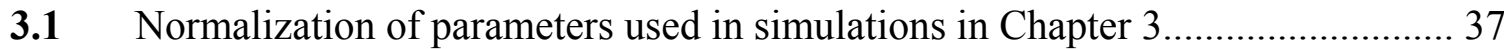

4.1 Normalization of parameters generally used in simulations in Chapter 4.............79 


\section{ACKNOWLEDGEMENTS}

I would gratefully and deeply thank my advisor, Prof. Yu U. Wang for his persistent guidance, encouragement, and support during my Ph.D. study, one year in Virginia Tech and three years in Michigan Tech. He taught me the essential knowledge beginning with the most fundamental mathematics and then gave me much freedom to explore my own. He also provides me many insightful suggestions which help me avoid lots of detours and mistakes in research. The methodology he taught me has greatly affected and will keep influence my academic career.

I would like to express my sincere gratitude to Prof. Yongmei M. Jin, for her guidance to me in the new field of ferromagnetic materials. She also assisted me greatly in the development of my DIFA code in parallel environment. Our collaboration is fruitful and partially reflected in a couple of coauthored publications.

I would like to thank Prof. Jaroslaw W. Drelich, Prof. Miguel Levy and Prof. Dennis Desheng Meng for their support as my committee and for helpful discussion on my research. I appreciate Prof. Mark R. Plichta, Prof. Stephen L. Kampe, Prof. Yun Hang Hu, Prof. Jiann-yang Hwang, Prof. Paul G. Sanders and Prof. Stephen A. Hackney for their friendship during my graduate studies. Thanks to Prof. Peter D. Moran for his suggestions and encouragement on my work. Thanks to Edward A Laitila, Owen P Mills and Ruth I. Kramer for their amicable help. I am also indebted to Ms. Allison M. Hein for polishing my draft paper.

I really enjoy the pleasant and happy days during the three years in Houghton with my labmates and friends. They are Jie Zhou, Fengde Ma, Liwei Geng, Ben Wang, Weilue He, Hang Zhang, Hui Wang and others cannot be listed completely. I enjoy the everyday lunch time with them together which makes me relaxed. Friendships with Joe Licavoli, Sanchai Kuboon are also sweet and memorable. 
Thanks to Dr. Debra Charlesworth (Graduate school) for carefully checking the format of this dissertation.

I also would like to express my deep gratitude to my parents and parents-in-law for their support and understanding during these years. Hope the accomplished thesis makes them feel delighted.

Financial support from National Science Foundation is gratefully acknowledged.

Finally, and most importantly, I would like to thank my wife Jane. Her support, patience and unswerving love despite our long distance were undeniably the bedrock of my life in recent years. 


\section{ABSTRACT}

Colloid self-assembly under external control is a new route to fabrication of advanced materials with novel microstructures and appealing functionalities. The kinetic processes of colloidal self-assembly have attracted great interests also because they are similar to many atomic level kinetic processes of materials. In the past decades, rapid technological progresses have been achieved on producing shape-anisotropic, patchy, core-shell structured particles and particles with electric/magnetic charges/dipoles, which greatly enriched the self-assembled structures. Multi-phase carrier liquids offer new route to controlling colloidal self-assembly. Therefore, heterogeneity is the essential characteristics of colloid system, while so far there still lacks a model that is able to efficiently incorporate these possible heterogeneities.

This thesis is mainly devoted to development of a model and computational study on the complex colloid system through a diffuse-interface field approach (DIFA), recently developed by Wang et al. This meso-scale model is able to describe arbitrary particle shape and arbitrary charge/dipole distribution on the surface or body of particles. Within the framework of DIFA, a Gibbs-Duhem-type formula is introduced to treat Laplace pressure in multi-liquid-phase colloidal system and it obeys Young-Laplace equation. The model is thus capable to quantitatively study important capillarity related phenomena.

Extensive computer simulations are performed to study the fundamental behavior of heterogeneous colloidal system. The role of Laplace pressure is revealed in determining the mechanical equilibrium of shape-anisotropic particles at fluid interfaces. In particular, it is found that the Laplace pressure plays a critical role in maintaining the stability of capillary bridges between close particles, which sheds light on a novel route to in situ firming compact but fragile colloidal microstructures via capillary bridges. Simulation results also show that competition between like-charge repulsion, dipole-dipole interaction and Brownian motion dictates the degree of aggregation of heterogeneously 
charged particles. Assembly and alignment of particles with magnetic dipoles under external field is studied. Finally, extended studies on the role of dipole-dipole interaction are performed for ferromagnetic and ferroelectric domain phenomena. The results reveal that the internal field generated by dipoles competes with external field to determine the dipole-domain evolution in ferroic materials. 


\section{Chapter 1. Introduction}

\subsection{Colloid as building block for advanced materials}

Colloidal particles dispersed in liquid phases have attracted interests for more than a century. With decreasing particle size, gravity decreases rapidly as particle mass (volume) is proportional to the cube of particle size thus usually gravity does not dominate individual particle motion. If the small particles adsorb a certain amount of charged ions, or are covered with a layer of polymer stabilizer (like a brush), then the electrostatic or steric repulsion is able to overcome van der Waals forces and Brownian motion to avoid spontaneous agglomeration of colloids. ${ }^{1}$ As a result, colloidal particles can be suspended in the carrier liquids for long time and the colloidal behavior can be tuned and controlled by modification on surface chemistry of particles or the carrier liquids, or by external electric/magnetic fields. ${ }^{2-4}$ Colloidal particle self-assembly has become an important bottom-up route to synthesize advanced materials with novel microstructures and functionalities $^{1-12}$ (Those artificial materials with unit cells smaller than external stimulus dimension are usually called metamaterials ${ }^{13,}{ }^{14}$ ). The rapidly developing technology allows the precise control of fine microstructures by colloidal self-assembly to compete even with nano-lithography, while compared with nano-lithography colloid self-assembly is unbeatable in terms of cost. ${ }^{8}$ Colloids have been employed as precursor for advanced materials, such as the photonic crystals. ${ }^{15}$ Colloidal crystals also offer great convenience for testing properties of photonic crystals. ${ }^{6}$ Colloid self-assembly is nowadays believed to be a promising and cost-effective route to build complete three-dimensional photonic crystals for micron or submicron wavelengths, which may offer omnidirectional photonic band gap for visible and infrared spectrum. ${ }^{16}$ Colloid self-assembly directed by liquid interfaces, inspired by Pickering emulsion, has recently attracted increasing interests. ${ }^{17-20}$ New shell-like soft solid materials, such the so called bijel ${ }^{21-23}$ and colloidosome ${ }^{7}$ (also 
called colloidal armour $^{24}$ ), were created. The colloidosome has found application in novel drug encapsulation and delivery. ${ }^{7}$

Colloid is also of great interests as a model system for studying a variety of kinetic processes in material science and fundamental condensed matter physics. An individual colloidal particle could be considered as an atom or a molecule and macroscopically colloids also exhibit phase behavior of gas, liquid, crystal, or amorphous solid. ${ }^{12,25,26}$ Colloid is thus a natural model system for studying materials science. The advantage of this model system is that the colloidal particles are much bigger than atoms and molecules thus much easier to observe. In addition, the mobility of a big colloidal particle is much lower than a small molecule, thus the kinetic process of colloid self-assembly is usually slow and subsequently the experimental observation does not require high time resolution. Studies on the crystalline nucleation and glass transition behavior in colloid science may shed light on the counterpart physical processes in condensed matter physics, while the latter is yet incompletely understood. ${ }^{12,27,28}$ Differences between molecules and the supermolecules (colloids) exist in interactions between colloidal particles and intermolecular forces. Intermolecular forces are well-known and they are listed as primary chemical bonds (covalent/ionic/metallic), hydrogen bond and van der Waals forces. Interparticle forces, however, can be more complicated (if we do not take them as summation of intermolecular forces anyway) in that the carrier liquid plays an important role. In addition to Coulombic forces and van der Waals forces, which depends on the properties of the carrier liquid, there are possibly steric forces (entropic forces), capillary forces (in multiphase fluid system), magnetostatic forces (for magnetic particles), and hydrophobic/hydrophilic forces (also known as solvation forces). ${ }^{1,29}$ The range and magnitude of each intermolecular force is typically not adjustable, whereas interactions between colloidal particles are tunable by a variety of external methods. The strength ranking of interparticle forces may even be changed. For example, density match and refractive index match of particles with the carrier liquids may eliminate effects of gravity and van der Waals forces. ${ }^{9,26}$ The density of surface charges can also be adjusted through chemical treatment. ${ }^{8,9}$ The steric repulsion between particles are changeable 
depending on the shape, size, elastic properties of particles and coatings. However, subsequent challenge for modeling colloid systems is also obvious due to the complexity of interparticle forces.

\subsection{Interparticle forces in colloidal systems}

For particles with charged surfaces, unless the carrier liquid is completely deionized and with low dielectric constant, the surfaces would adsorb ions of opposite charges from the liquid solution and forms electric double layer. ${ }^{29}$ The potential function describing the double layer related charge distribution obeys the Poisson-Boltzmann equation ${ }^{1,30}$ Solution for two particles of simple geometries has been extensively studied. Under low potential assumption $\left(q_{i} \psi \ll k_{B} T\right.$, where $q_{i}$ the unit charge quantity of species $i, \psi$ the electric potential, $k_{B}$ the Boltzmann constant and $T$ absolute temperature), the electric double layer generates a screened Coulomb potential $\psi$ which decays exponentially with respect to interparticle distance, i.e., $\psi \sim \psi_{0} e^{-\kappa r}$, with a characteristic decay length (Debye-Hückel length) of ${ }^{1,29}$

$$
\kappa^{-1}=\lambda_{D}=\sqrt{\frac{\varepsilon k_{B} T}{\sum_{i} n_{i}^{0} q_{i}^{2}}} .
$$

where $\varepsilon$ is the dielectric constant of the liquid and $n_{i}^{0}$ the mean number density of ion species $i$. The double layer repulsion has an effective range as short as a few Debye lengths, which can be as small as a few nanometers if the concentration of the electrolyte is high. In this case, if the size of colloidal particles is of tens of nanometers or above, the double layer repulsion can be well described by linear superposition approximation, ${ }^{30}$ thus multi-particle interaction can be simplified as sum of pairwise additive inter-particle repulsion. If, on the contrary, the carrier liquid is deionized then the Debye length approaches infinity thus double layer force reduces to fundamental Coulombic interaction. 
For particles separated at a gap distance of several nanometers, van der Waals forces can be significant. London dispersion force between two colloidal particles, which is sum of all intermolecular dispersion force, ${ }^{1}$ is due to induced-dipole-induced-dipole interaction which exists even between nonpolar molecules and it is largely the most important part of van der Waals forces (The other two types of van der Waals force are induction force and orientation force). ${ }^{29}$ Intermolecular van der Waals force is generally (but not always) described by a potential function proportional to the inverse sixth power of intermolecular distance. ${ }^{29}$ However, in solvent medium, van der Waals force is greatly reduced compared with that in free space. If the colloidal particles have dielectric constant identical to the carrier fluid, van der Waals forces can be absent. ${ }^{29}$ Moreover, van der Waals force has a retardation effect if interaparticle distance is large (typically 5 $\mathrm{nm}$ or above), which decreases the intermolecular potential from $1 / r^{6}$ to nearly $1 / r^{7}$ ( $r$ is the distance), thus in general van der Waals force is significant only for gap distance within a few nanometers. ${ }^{1,29}$ Combination of the double layer repulsion and van der Waals forces has successfully explained the stability of colloid system, which is attributed to the well-known DLVO theory, developed in the 1940s by Derjaguin, Landau, Verwey and Overbeek. ${ }^{31,32}$

Colloids are stabilized against agglomeration mainly through two repulsive effects. In addition to the aforementioned electric double layer repulsion, steric repulsion is another short range interaction frequently used to stabilize colloidal particles from coagulation. Steric repulsion can be introduced by coating particles with long chain polymer molecules where entropic forces keep particles away from each other at certain distance to prevent overlap of the polymer layers. ${ }^{29}$ The polymer chains can be grafted onto the particle surface to form a polymer hair. Steric force is shown to be related to the $\mathrm{pH}$ value, electrolyte concentration and amount of the polymer, while modeling on steric force is rather complex. ${ }^{33}$

When two particles are close to each other with a gap of a few nanometers, the accuracy of DLVO theory, which takes inter-particle force as a combination of van der 
Waals force and electric double layer force, is challenged ${ }^{30}$ since DLVO theory is essentially based on continuum assumptions. In addition, solvation forces can be very strong at very short range due to reorganization of the liquid molecules adjacent to the solid surface. ${ }^{1,29,30}$ Solvation force is generally a decaying oscillatory function of surface distance with periodicity of around molecular size according to experimental results in non-aqueous systems, ${ }^{29,30}$ which means multiple minimum of its potential exist at very short distance. In aqueous systems solvation force may not change its sign. ${ }^{29}$ Usually solvation force between close particles with hydrophilic surface is repulsive (often called hydration force) and with hydrophobic surfaces attractive (hydrophobic force).

If colloids are dispersed in a multiphase fluid system, capillary forces may play a significant role. If particles have comparable affinity to two fluid phases, at the inter-fluid interface the particles are trapped to reduce interfacial area (interfacial energy). For a particle of radius $R$, the free energy barrier to remove a particle from the interface is estimated to be $\mathrm{b}^{34}$

$$
\Delta G=\pi R^{2} \gamma(1 \pm \cos \theta)^{2},
$$

where $\gamma$ is interfacial tension, and $\theta$ contact angle. For particle of tens of nanomaters or above, such energy barrier is several orders of magnitude higher than characteristic thermal energy $k_{B} T$. In other words, particles will be almost irreversibly trapped at the liquid interface until the interface is filled. Particles adsorbed at liquid interfaces may be subject to lateral capillary interaction if the interface morphology is disturbed due to either external forces or contact angle restriction. For example, particles floated at flat liquid surface may be subject to attractive or repulsive lateral capillary forces due to surface disturbance by gravity, which is known as flotation force. ${ }^{35,36}$ Particles partially immersed in liquid film may be subject to attractive or repulsive lateral capillary forces due to capillary rise, which is known as immersion force. ${ }^{35,}{ }^{36}$ Capillary forces include two essential parts. One is interfacial tension, and the other is Laplace pressure. It needs to be emphasized that although the two parts are linked by Young-Laplace equation, they actually contribute separately and may take distinct roles. This is due to the fact that 
Laplace pressure is strongly dependent on the radius of curvature, which can be restricted by contact angle and boundary conditions in a confined geometry. For example, surface tension of small liquid bridges between two spherical particles attracts the particles, while Laplace pressure could either attract or repel the particles depending on the contact angle. ${ }^{37,38}$ With $90^{\circ}$ contact angle, surface tension attracts but Laplace pressure repels. Theoretical work on the lateral capillary forces is mostly based on spherical particles at equilibrium configuration. If particles are nonspherical or are moving, then capillary forces must be solved according to the real-time configuration, which relies largely on numerical methods.

Electrostatic and magnetostatic forces between colloidal particles exist when particles are with charges or magnetic dipoles. It needs to be emphasized that charge heterogeneity is important for colloidal particles. Atomic force microscopy results manifest surface heterogeneity on polystyrene spheres immersed in water and resultant heterogeneous surface charge distribution. ${ }^{39,40}$ Interactions due to charge heterogeneity can be orders of magnitude different from expectation by averaged charge. ${ }^{41}$ For simplicity, we consider the case that the carrier fluid is deionized (such as in polymer melt) so the electric double layer is absent (or the Debye length is infinity). For a particle with arbitrarily distributed charges, its electric potential function (outside the particle) can be written as multipole expansion of ${ }^{42}$

$$
\begin{aligned}
\varphi(\mathbf{r}) & =\varphi_{0}(\mathbf{r})+\varphi_{d}(\mathbf{r})+\varphi_{q}(\mathbf{r})+\ldots \\
& =\frac{1}{4 \pi \varepsilon}\left[\frac{Q}{r}+\frac{r_{i} d_{i}}{r^{3}}+\left(\frac{3 r_{i} r_{j}}{r^{5}}-\frac{\delta_{i j}}{r^{3}}\right) q_{i j}+\ldots\right]
\end{aligned}
$$

where summation convention is implied, $\delta_{i j}$ is the Kronecker delta, $\varepsilon$ the dielectric constant (assume homogeneous), and 


$$
\left\{\begin{array}{l}
Q=\int_{V} \rho\left(\mathbf{r}^{\prime}\right) d^{3} r^{\prime} \\
d_{i}=\int_{V} \rho\left(\mathbf{r}^{\prime}\right) r_{i}^{\prime} d^{3} r^{\prime} \\
q_{i j}=\frac{1}{2} \int_{V} \rho\left(\mathbf{r}^{\prime}\right) r_{i}^{\prime} r_{j}^{\prime} d^{3} r^{\prime}
\end{array}\right.
$$

are called monopole (net charge), dipole and quadrupole, respectively (Here $\rho\left(\mathbf{r}^{\prime}\right)$ is the charge density function, and higher order multipole terms are omitted). From Eq. (1.3) it can be concluded that in colloid system so long as interparticle distance is comparable with particle size, the electrostatic force between two heterogeneously charged particles does not equal that between two net charges, and even if net charge is zero, the point dipole-point dipole interaction is not in lieu of the superposition of all high order multipole interactions owing to finite dipole size. For colloid system, e.g., if the volume fraction of colloid particles is $10 \%$, the interparticle distance can be at most a couple of times of particle diameter (assuming spheres). When colloidal particles are densely distributed, especially if particles are nonspherical, the most commonly used point charge or point dipole model cannot describe the electrostatic interaction between particles, instead the long range and highly configuration dependent electrostatic interaction must be computed as a full spatial integral. ${ }^{41}$

In summary, at a distance as short as a few nanometers, interparticle forces are very complex and yet to be further studied, while for relatively larger length scale they are theoretically understood well. However, there is yet lack of study on the effect of nonspherical particle shape. For particles adsorbed at fluid-fluid interfaces, the geometry of particles affect interface morphology owing to contact angle restraints thus may alter capillary interaction between particles. The particle shape anisotropy may significantly alter the interparticle force distribution. In terms of particles of tens of nanometers or above, studying the shape anisotropy effect is expected to be more importance than, e.g., resolving the nano-scale surface potential of colloidal particles. The effect of charge heterogeneity is also to be further investigated, which not only helps to clarify those 
apparently abnormal phenomena, such as the like-charge attraction, ${ }^{39,} 43$ but also potentially provides new approach to controlling colloidal self-assembly.

\subsection{Numerical modeling methods on colloid self-assembly: A survey}

Theoretical models that can be solved typically are with simple geometries such as two particles with spherical shape, while for the assembly involved processes a certain amount of particles must be incorporated. Computer modeling and simulation in such cases is the first choice and it can also effectively complement experimental investigation and theoretical study. As a bottom-up approach to build structured materials, colloidal self-assembly requires micro- or meso-scale modeling tools so as to study the detailed mechanisms dominating collective colloidal behavior.

Molecular dynamics (MD) is such a method which defines intermolecular potential and meticulously calculates the trajectory of each molecule by Newtonian equations. In principle, MD should be able to capture all colloidal behavior from molecular level collision to macroscopic level phase behaviors giving sufficiently accurate intermolecular potential function. The intermolecular potential is usually "hard" such as the LennarJones potential, which limits the time step of molecular dynamics to be very small. Therefore, owing to the limited computational capability conventional MD is only able to handle microscopic processes of small length scale and time scale. For physical processes of long chain polymers, the vibration frequency of bond lengths and bond angles can be 6 orders of magnitude higher than the torsional rotation of whole molecules. ${ }^{44}$ Such disparate time scales significantly hinder application of atomic scale MD modeling. For colloid system involves a relatively huge number of atoms and long time evolution, conventional MD modeling at atomic level is virtually impractical.

A realistic approach to modeling colloid system is using coarse-graining method in which "super-atoms" are constructed substituting atomic level molecules and empirical pair potential between the "super-atoms" is usually employed. ${ }^{45}$ Dissipative particle 
dynamics (DPD) employs a set of DPD particles to represent every small region of fluid, and separates inter-particle forces into conservative, dissipative, and random forces. ${ }^{46,47}$ The latter two forces combine to simulate Brownian motion and maintain system temperature. In DPD particles can move continuously off-lattice and momentum is locally conserved so as to capture fundamental hydrodynamic behavior. The inter-particle potential in DPD is much softer than MD, therefore its time step is two or three orders of magnitude larger than conventional MD. ${ }^{47}$ DPD has been applied to simulate phase behavior of spherical nano-colloids. ${ }^{46}$ Yan et al used a number of DPD particles bonded together to mimick Janus nanoparticles and simulated self-assembly of Janus nanoparticles to form nanocomposites. ${ }^{48}$

Monte Carlo (MC) method is a popular statistical approach which casts the physical process by repeating stochastic numbers and the system is updated from one state to the next by probability matrices. ${ }^{44} \mathrm{MC}$ is also able to avoid depicting the extremely detailed atomic level motion of molecules. Compared with MD, MC models evolve the system by reference of macroscopic energy level, thus MC is more capable of capturing macroscopic and long time scale processes than $\mathrm{MD}$, though it cannot quantitatively study the time dependence. In order to handle larger length scales, coarse-grained particles are also frequently used in MC modeling instead of atomistic particles. ${ }^{49}$ Selfassembly of amphiphilic nanoparticles and diblock copolymers by MC have been reported ${ }^{49,50}$

Colloid system is still a great challenge for the continuum based computational fluid dynamics (CFD). However, the complex boundary conditions associated with a large number of moving particles in colloid system make applications of continuum based CFD models to colloid system yet computationally intractable. An alternative approach to solving the Navier-Stokes equation is the Lattice Boltzmann method (LBM, proposed just a couple of decades ago $)^{51-53}$ which has demonstrated capability to treat hydrodynamics with complex geometric boundary conditions. Based on the Boltzmann equation, LBM employs fictitious fluid particles and allow them to propagate or collide on fixed mesh 
maintaining mass and momentum conservation. LBM has been proven to be convergent to solution of Navier-Stokes equation. ${ }^{51,54}$ Its precision, however, depends on the discrete mesh grid. Therefore, the hydrodynamic lubrication effect, which appears when particles come into close proximity, entails extra care in LBM. ${ }^{55}$ Combined with Cahn-Hilliard models, LBM has been successfully applied to simulate binary liquid - solid contact line motion and spherical colloid self-assembly in multiphase fluid system, ${ }^{21,53}$ while in contrast the continuum based coupled Cahn-Hilliard-Navier-Stokes models have been reported only on simple two-phase flows without colloidal particles. ${ }^{56-59}$ Nevertheless, study of moving particles with anisotropic shapes has not been reported by LBM either.

Actually, for colloidal liquid system the Reynolds number is typically very low, such that the viscous force dominates over inertia force. Without inertia term, Navier-Stokes equation reduces to (Stokes equation)

$$
\left\{\begin{array}{l}
\nabla \cdot \mathbf{v}=0 \\
\nabla p=\mu \nabla^{2} \mathbf{v}
\end{array}\right.
$$

where $\mathbf{v}$ is fluid velocity, $\mu$ dynamic viscosity of fluid and $p$ the hydrodynamic pressure. The Stokes equation is linear and independent of time, thus the described fluid is creeping flow when particles are moving and quiescent otherwise. Therefore, the resultant hydrodynamic resistant force (torque) imposed on a particle can be linked to particle velocity (angular velocity) through mobility matrices. ${ }^{60}$ It is the Stokes dragging force that dominates particle resistance while the mobility tensors depend only on the geometry of particle ${ }^{60,61}$ Extensive studies have been reported on the mobility of particles in Stokes flow. ${ }^{1,60,62,63}$

Diffuse interface field approach (DIFA) is a novel mesoscale method to simulate arbitrarily shaped colloids, first developed by Wang. ${ }^{64}$ Using template fields to depict the geometry of each particle and dealing with the contact force by using the natural gradient of diffuse interfaces without explicitly tracking interfaces, DIFA is able to efficiently calculate short range steric forces, long range electrostatic/magnetostatic forces, capillary force (when particles are dispersed in multiphase fluid system), Brownian forces and it is 
open to include other pairwise additive interparticle forces. ${ }^{37,}{ }^{64-66}$ In DIFA-colloid models, the kinetic equations of particles are simplified according to the laws of low Reynolds number hydrodynamics. The velocity and angular velocity of particles are linked to resistant force and torque by the mobility matrices, thus fluid dynamics is not solved. ${ }^{64,66}$ DIFA has been applied to study charged colloids, ${ }^{66}$ dipolar colloids, ${ }^{64,} 67$ shape anisotropy effects and charge heterogeneity effects. ${ }^{67}$ The softened interparticle potential (in close contact) effectively overcomes convergence difficulties that are frequently met in sharp interface numerical models, just as dissipative particle dynamics is able to employ a much larger time step than conventional molecular dynamics .

It needs to be mentioned that modeling work on shape anisotropic particles has been attempted by using frozen clusters of spherical particles (coarse-grained "molecules") to mimick patchy and nonspherical colloidal particles. ${ }^{45,68}$ Such processing method, however, obviously has a lower precision in resolving particle shape than DIFA, considering that DIFA has spatial resolution higher than grid size. ${ }^{64-66}$ Moreover, in case of interfacial tension existing in multiphase liquid colloid system, the rugged surface of colloids composed of clustered spheres may lead to severe contact angle hysteresis, ${ }^{69,70}$ although such models have been reported to model multiphase liquid colloid system.

\subsection{DIFA to modeling self-assembly of heterogeneous colloidal particles and dipole-dipole interaction related ferroic domain evolution}

So far most literatures on colloid system focused on spherical particles, both in experimental work and in theories. However, in reality the particles cannot be ideally monodispersed spheres, and polydispersity in shape and in size is inevitable. Polydispersity is known to greatly affect the kinetic processes of self-assembly, such as nucleation and glass transition. ${ }^{25,28}$ On the other hand, spherical particles have a lot of limitations. For example, homogeneous spherical colloids easily crystallize into f.c.c. lattice which, however, does not produce complete photonic band gap. ${ }^{6}$ Fortunately, 
individual particles as the basic building block of colloidal assembly have no longer been restricted to simple spherical shape owing to rapid progress over the past decade in processing techniques to fabricate shape-anisotropic, patchy, and core-shell structured particles. ${ }^{4}$ 10-12 Particles with anisotropic shapes and/or heterogeneous properties (such as nonuniform charge distribution, heterogeneous affinity to liquid phases, and different functionalities of core and shell materials) have greatly enriched the self-assembled structures. ${ }^{4,6,11,12,45,71-73}$ Modeling colloidal particles with any anisotropic shape is the strength of DIFA.

The aforementioned charge heterogeneity effect plays an important role in colloid self-assembly and is to be modeled and studied by using DIFA. In previous simulation work (e.g., coarse-grained MD or DPD), each particle was typically treated as a simple point charge or point dipole. The heterogeneous charge distribution on colloidal particles was thus rarely considered. When colloidal particles are densely distributed, the commonly used point charge or point dipole model cannot describe the electrostatic interaction between particles. DIFA uses a spectral Fourier method to calculate the long range electrostatic force between any heterogeneously charged particles which is equivalent to integrate the electrostatic interaction in full space. ${ }^{41,64,66}$

This thesis focuses on modeling colloid system by DIFA with emphasis on studying anisotropic particle shapes, heterogeneous charge distribution, capillarity effects or a combination of them. From the scientific standpoint, this work is motivated by (1) Develop an effective model to include particle shape anisotropy with reasonable computational cost, (2) Develop an effective model to include Laplace pressure, which combining with interfacial tension takes a dominant role in multiphase-liquid colloid system, (3) Develop an effective model to incorporate charge heterogeneity and its influence on colloid aggregation and self-assembly. If we take the external field of a particle with heterogeneous charge distribution as a superposition of multipole expansion, except for the net charge field, the dipole field, quadrupole field and higher order terms are strongly orientation dependent, thus the orientation or rotation of the particles is 
important. The shape anisotropy of particles also leads to distorted electric field distribution near particle surface and orientation dependent short range interaction. The effects on dipole-dipole interaction and combinatorial interaction between net charges and dipoles are to be revealed.

As a meso-scale simulation method, DIFA typically applies to colloids with size of tens of nanometers or above. In this thesis, our DIFA model focuses on calculating long range electrostatic/magnetostatic forces and capillary forces while including effective short range repulsion. DIFA can efficiently handle short range interparticle forces by using the intrinsic properties of diffuse interface without explicitly tracking the interfaces. Despite the fact that short range force between nano-spaced particles is very complex and yet a challenge for any model (as stated before), in this work the short range force is simply treated as a monotonic repulsion. Our DIFA model focuses on modeling shape anisotropy and charge heterogeneity effects and capillarity phenomena which is on the length scale of particle size and is thus of more essential importance than the smaller length scale interparticle forces (such as the aforementioned solvation force) for the colloid systems of interests.

In Chapter 2, a full description of our DIFA model is presented and some benchmark simulations are performed for model verification. In Chapter 3, capillary interaction between shape-anisotropic particles is investigated and especially the role of Laplace pressure is revealed. In particular, the Laplace pressure takes a critical role in determining stability of capillary bridges between colloidal particles, which can be utilized to firm fragile compact colloidal microstructures. In Chapter 4, self-assembly of colloidal particles with heterogeneous charge distribution and dipolar particles is simulated. Ordered and disordered (aggregation) microstructures are formed in various conditions, such as with/without applied external field, particle volume fraction, degree of charge heterogeneity and temperature etc. 
In the last chapter, extended studies are performed on dipole-dipole interaction which also plays an important role in functional ferroelectric/ferromagnetic materials. As a diffuse-interface method (in contrast to sharp-interface method such as Volume of Fluid and Level Set method, etc), ${ }^{59}$ DIFA is a generalized phase field method. The latter has been widely applied to model materials with complex microstructures and their evolution, ${ }^{74,75}$ and it has demonstrated its unique strength on modeling ferroelectric and ferromagnetic domain evolution. Although kinetic processes are different in ferroic domain evolution from in colloidal self-assembly, similarity can be found in determining system evolution as a result of free energy minimization, especially when electrostatic / magnetostatic energy dominates. Modeling and simulation on ferroelectric and ferromagnetic domain phenomena are presented and the underlying mechanism of various dipole-dipole interaction involved domain evolution is revealed. In either multiphase liquid colloid system or ferroelectric/ferromagnetic system with multidomains, DIFA (phase field) models exhibit powerful capability to simulate complex microstructure evolutions. 


\section{Chapter 2. Modeling method}

For multi-phase liquid colloid system, the Laplace pressure is formulated within the framework of DIFA and it is verified by agreement with Young-Laplace equation. Other formula of DIFA to modeling colloidal system have been developed by Wang and Millett, ${ }^{64-66}$ and the corresponding algorithms are narrated following their work.

\subsection{DIFA to modeling colloidal particles in multiphase liquid colloid system: the Laplace pressure}

\subsubsection{DIFA formulation of free energy}

In DIFA model of multi-liquid-phase colloid system, each fluid phase is described by one concentration field variable $c_{\alpha}$ and each solid particle by one field $\eta_{\beta}$. The total system free energy assumes the following form

$$
F=\int\left[f\left(\left\{c_{\alpha}\right\},\left\{\eta_{\beta}\right\}\right)+\sum_{\alpha} \frac{1}{2} \kappa_{\alpha}\left|\nabla c_{\alpha}\right|^{2}\right] d V
$$

where $f\left(\left\{c_{\alpha}\right\},\left\{\eta_{\beta}\right\}\right)$ is the nonequilibrium local bulk chemical free energy density that defines the thermodynamic properties of a multi-phase system consisting of two liquid phases $(\alpha=1,2)$ and $N$ solid particles $(\beta=1, N)$, and is expressed as

$$
f\left(\left\{c_{\alpha}\right\},\left\{\eta_{\beta}\right\}\right)=A\left[\sum_{\alpha=1}^{2}\left(3 c_{\alpha}^{4}-4 c_{\alpha}^{3}\right)+\sum_{\beta}\left(3 \eta_{\beta}^{4}-4 \eta_{\beta}^{3}\right)+6\left(\chi c_{1}^{2} c_{2}^{2}+\sum_{\beta} \sum_{\alpha=1}^{2} \lambda_{\alpha} c_{\alpha}^{2} \eta_{\beta}^{2}\right)\right]
$$

This Landau-type free energy function is phenomenological in nature to reproduce the correct energy landscape with minima at $\left\{c_{\alpha=i}=1, c_{\alpha^{\prime} \neq i}=0, \eta_{\beta}=0\right\}$ for liquid phase $i$ and $\left\{c_{\alpha}=0, \eta_{\beta=j}=1, \eta_{\beta^{\prime} \neq j}=0\right\}$ for solid particle $j$. In general, for partially miscible binary 
liquids with a miscibility gap, for convenience the concentrations $c_{1}$ and $c_{2}$ are defined as molar fractions of the two liquid phases with respective equilibrium concentrations $c_{\mathrm{A} 1}$ and $c_{\mathrm{A} 2}$ in terms of component $\mathrm{A}$ (or $c_{\mathrm{B} 1}$ and $c_{\mathrm{B} 2}$ in terms of component $\mathrm{B}$ ), thus $c_{1}+c_{2}$ $=1$ and the true local concentration in terms of components $\mathrm{A}$ and $\mathrm{B}$, respectively, is

$$
c_{\mathrm{A}}=c_{1} c_{\mathrm{A} 1}+c_{2} c_{\mathrm{A} 2} \quad c_{\mathrm{B}}=c_{1} c_{\mathrm{B} 1}+c_{2} c_{\mathrm{B} 2}
$$

The gradient terms in Eq.(2.1) describe the energy contributions from liquid-liquid and liquid-solid interfaces. As a result, all field variables $\left\{c_{\alpha}\right\}$ smoothly transit from 1 to 0 forming diffuse interfaces at both liquid-liquid interfaces and liquid-solid interfaces. Also, $\eta_{\beta}=1$ inside the particle $\beta$ and $\eta_{\beta}=0$ outside, and the fields $\left\{\eta_{\beta}\right\}$ also smoothly transit through diffuse interfaces. Inside liquids $\left(\eta_{\beta}=0\right)$, the free energy function in Eq.(2.2) describes a double-well potential for binary solution with a miscibility gap. The constant $A$ is an energy scaling coefficient, and the parameters $\chi, \lambda_{\alpha}$ and $\kappa_{\alpha}$ are used to control the fluid-fluid and fluid-solid interfacial energy densities. Thus, the model is able to simulate colloidal particles of different wettabilities.

\subsubsection{Laplace Pressure}

Across a curved liquid interface, there exists a pressure jump (Laplace pressure), which is determined by the well-known Young-Laplace equation. According to thermodynamics, the pressure variation leads to chemical potential variations in the two bulk phases which obey the Gibbs-Duhem relation (under isothermal condition) ${ }^{76}$

$$
c_{\mathrm{A}} d \mu_{\mathrm{A}}+c_{\mathrm{B}} d \mu_{\mathrm{B}}=d p,
$$

where $c_{\mathrm{A}}$ and $c_{\mathrm{B}}$ are the true local concentration respectively in terms of components $\mathrm{A}$ and $\mathrm{B}$ as given in Eq.(2.3), $\mu_{\mathrm{A}}=\partial f / \partial c_{\mathrm{A}}$ and $\mu_{\mathrm{B}}=\partial f / \partial c_{\mathrm{B}}$ are the chemical potentials in the bulk, and $f\left(c_{\mathrm{A}}, c_{\mathrm{B}}\right)$ is the free energy density function. Using chain rules of differentiation yields 


$$
\mu_{\mathrm{A}}=\frac{\mu_{1} c_{\mathrm{B} 2}-\mu_{2} c_{\mathrm{B} 1}}{c_{\mathrm{A} 1} c_{\mathrm{B} 2}-c_{\mathrm{A} 2} c_{\mathrm{B} 1}}, \quad \mu_{\mathrm{B}}=\frac{\mu_{2} c_{\mathrm{A} 1}-\mu_{1} c_{\mathrm{A} 2}}{c_{\mathrm{A} 1} c_{\mathrm{B} 2}-c_{\mathrm{A} 2} c_{\mathrm{B} 1}},
$$

where $\mu_{\alpha}=\partial f / \partial c_{\alpha}$ (in the bulk). For the free energy function defined in Eq (2.2), one obtains

$$
\frac{\partial f}{\partial c_{i}}=12 A\left[c_{i}^{3}-c_{i}^{2}+B_{12} c_{i} c_{3-i}^{2}+\sum_{\beta=1}^{n} D_{i} c_{i} \eta_{\beta}^{2}\right]
$$

Eq. (2.4) can be rewritten with respect to the equilibrium values $\mu_{\mathrm{A}}^{0}, \mu_{\mathrm{B}}^{0}$ and $p^{0}$ in the case of flat interface as

$$
c_{\mathrm{A}}\left(\mu_{\mathrm{A}}-\mu_{\mathrm{A}}^{0}\right)+c_{\mathrm{B}}\left(\mu_{\mathrm{B}}-\mu_{\mathrm{B}}^{0}\right)=p-p^{0} .
$$

For the free energy function given in Eq. (2.2), $\mu_{1}^{0}=\mu_{2}^{0}=0$, and according to Eq. (2.5), $\mu_{\mathrm{A}}^{0}=\mu_{\mathrm{B}}^{0}=0$, which simplifies Eq. (2.7) to

$$
p-p^{0}=c_{\mathrm{A}} \mu_{\mathrm{A}}+c_{\mathrm{B}} \mu_{\mathrm{B}}
$$

Substituting Eq. (2.5) and Eq. (2.3) into Eq. (2.8) gives

$$
p-p^{0}=c_{1} \mu_{1}+c_{2} \mu_{2}
$$

which can be conveniently evaluated from the free energy function . That is, the GibbsDuhem equation also holds for our phenomenological Landau-type free energy function, which can be understood by thinking in terms of liquid phases 1 and 2 instead of components A and B in the liquid. In particular, the Laplace pressure causes changes in the chemical potentials through slight shifts in compositions away from their equilibrium values. Assuming $c_{1}$ and $c_{2}$ as equilibrium values in Eq. (2.9) gives $p-p^{0}=\mu_{\alpha}$ in each liquid phase $\alpha$. Using this relation, the Cahn-Hilliard equation is in effect equivalent to $\mathbf{J} \propto-\nabla p$, i.e., liquid flow flux is driven by the gradient of pressure, which is phenomenologically equivalent to Darcy's law. 


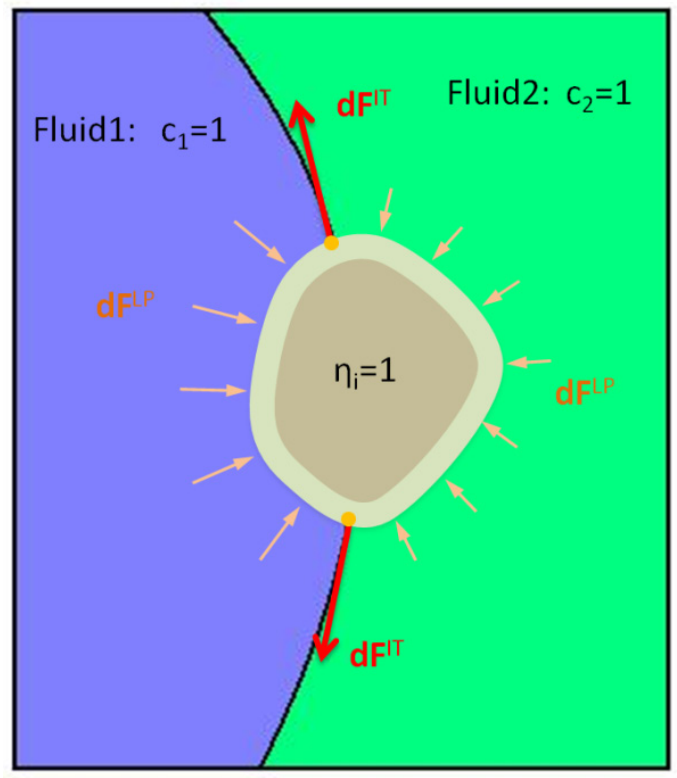

Figure 2.1 Schematic of Laplace pressure and interfacial tension imposed on a particle adsorbed at liquid-liquid interface

Laplace pressure associated with curved liquid interface can be determined from the changes in chemical potentials by using Eq. (2.8) and, in computer simulation, using Eq. (2.9). On the other hand, Laplace pressure is governed by Young-Laplace equation, which in two-dimensional case is

$$
\Delta p=\gamma / R
$$

where $\gamma$ is interfacial tension, and $R$ is radius of curvature of the curved interface. Eq. (2.10) can be used to verify the pressure as determined in computer simulation from Eq. (2.9). Figure 2.2 shows the computational results, where a single liquid droplet of phase 1 immerged in phase 2 is simulated to reach equilibrium. The internal pressure $p_{1}$ and external pressure $p_{2}$ of the droplet are calculated by using Eq. (2.9), and the pressure difference $\Delta p=p_{1}-p_{2}$ across the liquid interface (i.e., Laplace pressure) is compared with Eq. (2.10). Droplets of different radii and interfacial tensions, adjusted through the parameters $\kappa_{\alpha}$ in Eq. (2.1), are simulated. For all simulated cases, good agreements are obtained between Eq. (2.9) and Eq. (2.10), as shown in Figure 2.2, verifying the GibbsDuhem-type thermodynamic treatment of Laplace pressure within our phenomenological 
DIFA model of multi-liquid-phase colloidal system. It also shows that the solution of the diffuse interface model is consistent with the exact solution at sharp-interface limit.

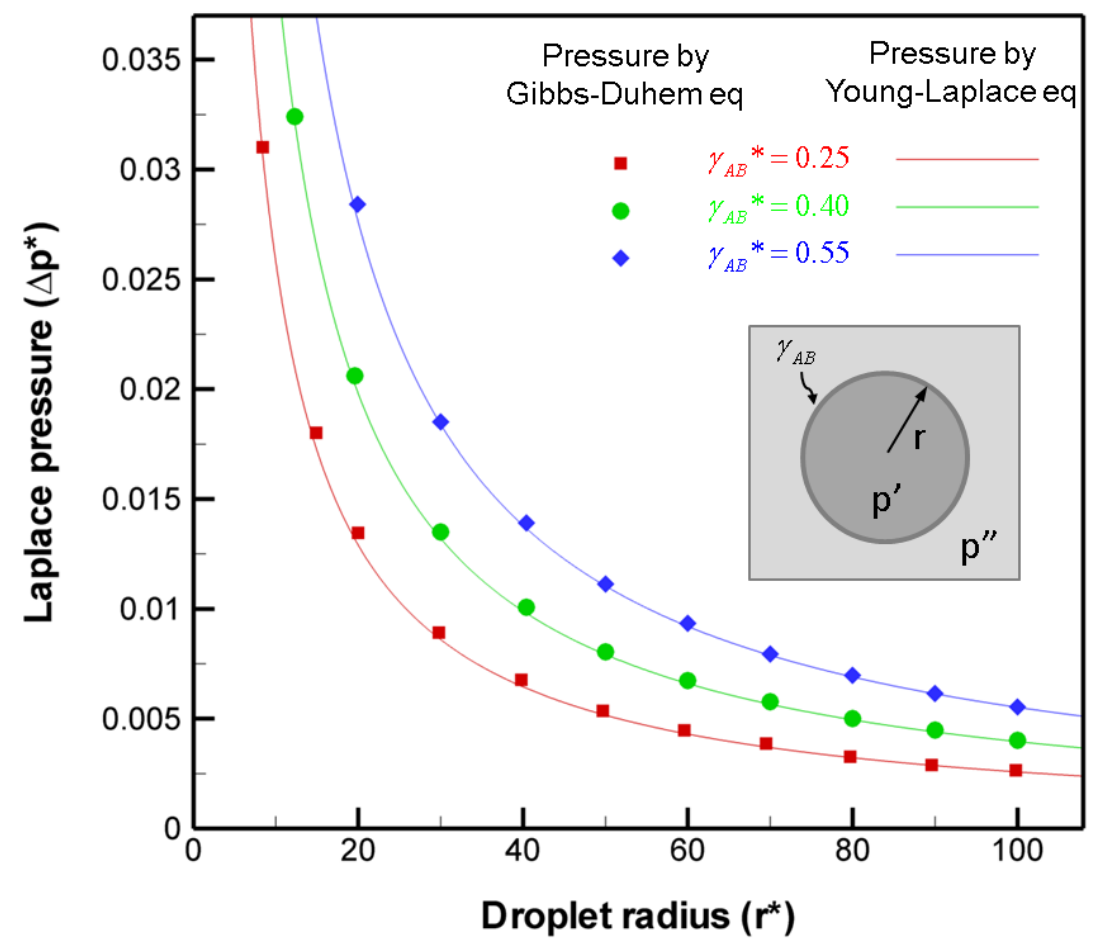

Figure 2.2 Comparison of Laplace pressure across a curved liquid interface as determined from Gibbs-Duhem relation with that determined from Young-Laplace equation.

It is worth noting that, as any field variables in DIFA, the pressure determined from Gibbs-Duhem relation (Eq. 2.9) also transits smoothly but rapidly across curved interfaces, as shown in Figure 2.3, whose jump gives the Laplace pressure in agreement with the Young-Laplace equation. The pressure within the interface zones does not yet have rigorous physical definition, while it can be understood by interpolation of the pressure jump across the two liquid phases. The simple Gibbs-Duhem relation based formula in the DIFA model is applicable to arbitrarily complex multi-phase morphologies without explicitly tracking the moving interfaces, while direct application of YoungLaplace equation to determine the Laplace pressure in such situations would be very 
difficult. Therefore, treatment of Laplace pressure is an important advance in DIFA model development.
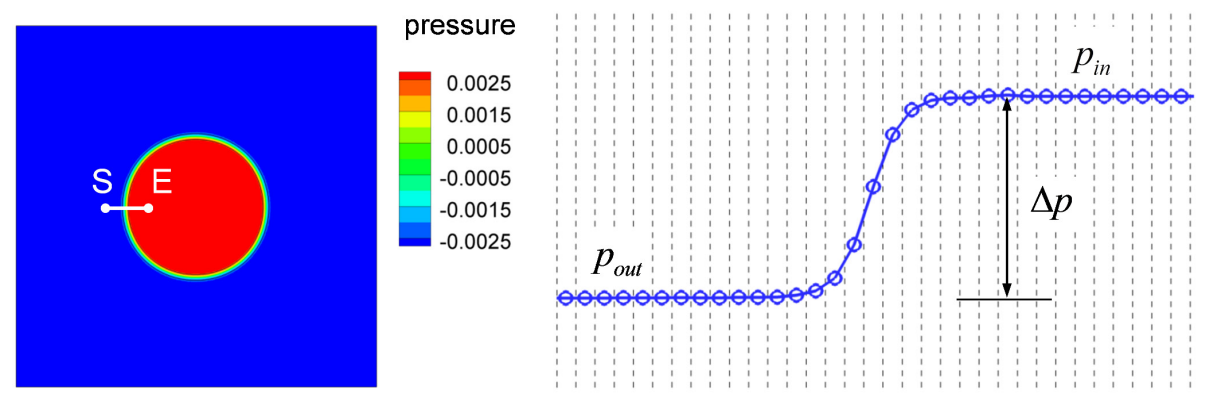

Figure 2.3 Pressure change along a path $\overrightarrow{S E}$ across a curved liquid interface from outside a droplet to inside, as determined by Gibbs-Duhem relation. Pressure is uniform inside and outside the droplet, while smoothly but rapidly transits across the diffuse interface.

\subsection{Steric interaction, electrostatic/magnetostatic interaction and Brownian forces}

Colloids are stabilized against agglomeration usually through two repulsive effects. One is steric repulsion ${ }^{33,77}$ typically by coating particles with long chain polymer molecules where entropic forces keep particles away from each other at certain distance to prevent overlap of the polymer layers, as schematically illustrated in Figure 2.4(a). The other is electrostatic repulsion resulting from electric double layer for particles with adsorbed charges on surfaces. ${ }^{30}$ In this work, we consider colloidal particles stabilized by steric forces, while charged particles have been previously considered using similar DIFA model ${ }^{66}$ The coating of polymer molecules is described by introduction of an additional "halo" field variable $\tilde{\eta}_{\beta}$ for particle $\beta$ in addition to the field $\eta_{\beta}$, as shown in Figs. 2.4(b) and 2.4(c) for a pentagon-shaped particle. The halo field $\tilde{\eta}_{\beta}$ is bound to the field $\eta_{\beta}$ and they together describe the particle as well as evolve together through translational and 
rotational rigid body motions. The short-range force of steric repulsion between particles is treated through local force density acting on particle $\beta$ defined as ${ }^{64}$

$$
d \mathbf{F}^{\mathrm{SR}}(\mathbf{r}, \beta)=\kappa_{\mathrm{S}} \sum_{\beta \neq \beta^{\prime}} \tilde{\eta}(\mathbf{r}, \beta) \tilde{\eta}\left(\mathbf{r}, \beta^{\prime}\right)\left[\nabla \tilde{\eta}(\mathbf{r}, \beta)-\nabla \tilde{\eta}\left(\mathbf{r}, \beta^{\prime}\right)\right] d V
$$

$\kappa_{\mathrm{S}}$ is a force constant to adjust the "stiffness" of the steric repulsion. Physically the repulsive steric forces keep particles from agglomeration. In numerical simulations, the steric repulsion prevents particles from penetrating into each other and also maintains a certain distance between jammed particles at fluid interfaces so that the capillary forces can be treated accurately.

(a)

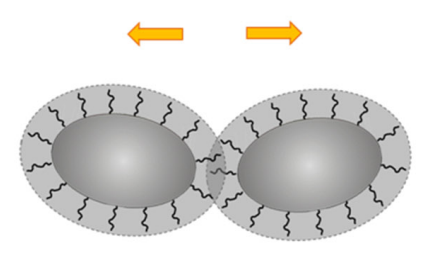

Steric repulsion
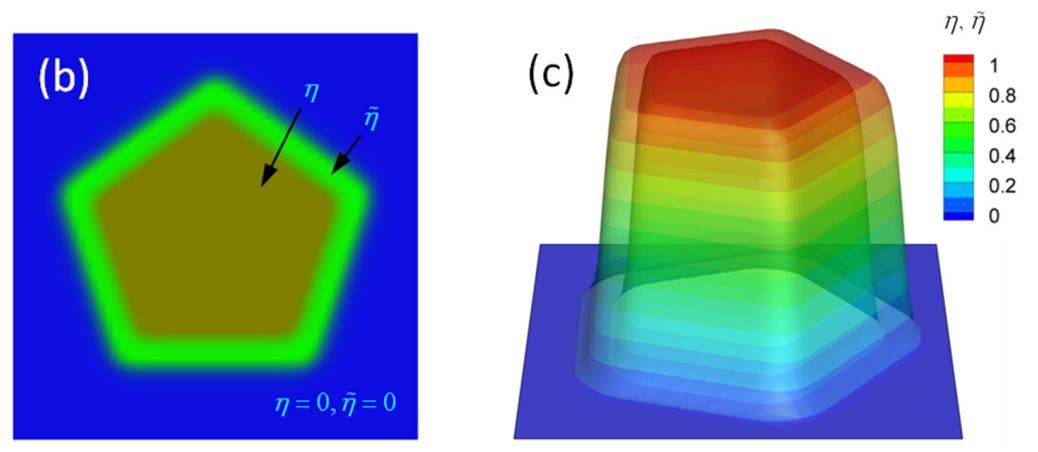

Figure 2.4 (a) Schematic of steric repulsion between two particles. (b) Two order parameters (field variables) $\eta$ and $\tilde{\eta}$ are used to describe the "core" and "halo" of one coated particle of pentagon shape. (c) 3D visualization (height representing the value) of the order parameter distributions of $\eta$ and $\tilde{\eta}$. Both field variables smoothly but rapidly transit from 1 to 0 across diffuse interfacial zones of the particle.

Laplace pressure is an important contribution to capillary forces (the other contribution is from interfacial tension). For example, in pendular capillary bridges, the negative Laplace pressure attracts the two particles together whose effect is even more significant than the surface tension itself. ${ }^{37,38,78}$ For particles adsorbed at curved liquid interfaces, Laplace pressure also plays an important role in determining the equilibrium interface configuration. To take into account the force on particle $\beta$ from Laplace 
pressure in liquid phases, a Laplace pressure force term is introduced through local force density defined as

$$
d \mathbf{F}^{\mathrm{LP}}(\mathbf{r}, \beta)=\kappa_{\mathrm{P}} \nabla \tilde{\eta}(\mathbf{r}, \beta) p(\mathbf{r}) d V,
$$

where the pressure field $p(\mathbf{r})$ is determined by using Eq. (2.9) while the constant pressure $p^{0}$ does not contribute to Laplace pressure, and $\nabla \tilde{\eta}$ defines the inward normal direction of the particle surface. Using the "halo" field $\tilde{\eta}$ instead of $\eta$ to evaluate the Laplace pressure force gives more reliable and accurate results in numerical simulations. The force constant $\kappa_{\mathrm{P}}$ is calibrated in simulations, as presented in next Section.

The interfacial tension imposed on particle $\beta$ is formulated by ${ }^{65}$

$$
d \mathbf{F}^{\mathrm{IT}}(\beta)=\kappa_{\mathrm{T}}\left[\nabla c \times\left(\nabla c \times \nabla \tilde{\eta}_{\beta}\right)\right] d V=\kappa_{\mathrm{T}}\left[\left(\nabla c \cdot \nabla \tilde{\eta}_{\beta}\right) \nabla c-|\nabla c|^{2} \nabla \tilde{\eta}_{\beta}\right] d V,
$$

where $\nabla c=c_{1} c_{2}\left(\nabla c_{1}-\nabla c_{2}\right)$. The force constant $\kappa_{\mathrm{T}}$ is determined in accordance with liquid interfacial tension, i.e., the integral of interfacial tension force at a fluid-fluidparticle contact zone reproduces $\gamma_{12}$, thus $\kappa_{\mathrm{T}}$ is determined for the chosen values of the coefficients in the free energy function (Eq 2.1 and Eq 2.2).

\section{Brownian motion}

Brownian motion of colloidal particles can be taken as a Markov process, which means the position of a particle at time step $\mathrm{n}+1$ solely depends on its previous position at time step $\mathrm{n}^{44}$ Statistical analyses give the description of Brownian motion as a cast of Gaussian-distributed Langevin noises (Brownian forces). The noise obeys uncorrelated Gaussian distribution with zero mean and its variance is determined by the fluctuationdissipation theorem, given by ${ }^{79}$

$$
\left\{\begin{array}{l}
<\xi_{i}(t) \xi_{j}\left(t^{\prime}\right)>=2 k_{B} T \delta\left(t-t^{\prime}\right) / M_{i j} \\
<\xi_{i}(t)>=0
\end{array}\right.
$$

In numerical simulation, it is 


$$
<\xi_{i} \xi_{j}>=\frac{2 k_{B} T}{M_{i j} \Delta t}
$$

where $M_{i j}$ is the mobility tensor of particles, and $\Delta t$ the time step. In case of $M_{i j}=M \delta_{i j}$, the standard deviation of noise $\xi_{i}$ is given by

$$
\sigma_{\xi}=\sqrt{\frac{2 k_{B} T}{M \Delta t}}
$$

The Brownian force is small compared with capillary forces for particles of micron size or above, as has been stated in Chapter 1.

\section{Electrostatic/magnetostatic interaction}

The electric field due to heterogeneously distributed charge on colloids is calculated in Fourier space by ${ }^{66}$

$$
\mathbf{E}(\mathbf{r})=\mathbf{E}^{e x}-\frac{i}{\varepsilon} \int \frac{d^{3} k}{(2 \pi)^{3}} \frac{\tilde{\rho}(\mathbf{k})}{k} \mathbf{n} e^{i \mathbf{k} \cdot \mathbf{r}}
$$

where $\tilde{\rho}(\mathbf{k})$ is Fourier transform of charge distribution function $\rho(\mathbf{r})$, and $\mathbf{n}=\mathbf{k} / k$ is a unit vector in k-space. The density of electrostatic force imposed on the $\alpha$ th particle is

$$
d \mathbf{F}^{e l}(\alpha)=\mathbf{E}(\mathbf{r}) \rho(\mathbf{r} ; \alpha) d V
$$

and the density of torque (about center of mass of the particle) is

$$
d \mathbf{T}^{e l}(\alpha)=\left[\mathbf{r}-\mathbf{r}^{c}(\alpha)\right] \times \mathbf{E}(\mathbf{r}) \rho(\mathbf{r} ; \alpha) d V
$$

where $\mathbf{r}^{c}(\alpha)$ is the position of the center of mass of the $\alpha$ th particle. The charge distribution function can be linked to the order parameter of particles, e.g.,

$$
\rho(\mathbf{r} ; \alpha)=\rho_{0} \eta(\mathbf{r} ; \alpha)
$$

such that any heterogeneous charge distribution associated with particle $\alpha$ can be described by the field variable $\eta(\mathbf{r} ; \alpha)$.

In terms of polarization field, the generated electric field is expressed as ${ }^{64}$ 


$$
\mathbf{E}(\mathbf{r})=\mathbf{E}^{e x}-\frac{1}{\varepsilon} \int \frac{d^{3} k}{(2 \pi)^{3}}[\mathbf{n} \cdot \tilde{\mathbf{P}}(\mathbf{k})] \mathbf{n} e^{i \mathbf{k} \cdot \mathbf{r}}
$$

where $\tilde{\mathbf{P}}(\mathbf{k})$ is Fourier transform of polarization distribution function $\mathbf{P}(\mathbf{r})$. The electric field generates electrostatic force density on the $\alpha$ th particle of

$$
d \mathbf{F}^{e l}(\alpha)=[\mathbf{P}(\mathbf{r} ; \alpha) \cdot \nabla] \mathbf{E}(\mathbf{r}) d V
$$

and torque density of

$$
d \mathbf{T}^{e l}(\alpha)=\mathbf{P}(\mathbf{r} ; \alpha) \times \mathbf{E}(\mathbf{r}) d V+\left[\mathbf{r}-\mathbf{r}^{c}(\alpha)\right] \times\{[\mathbf{P}(\mathbf{r} ; \alpha) \cdot \nabla] \mathbf{E}(\mathbf{r})\} d V
$$

The polarization field $\mathbf{P}(\mathbf{r} ; \alpha)$ of particle $\alpha$ is predefined and move together with the particle and it can be, for example,

$$
\mathbf{P}(\mathbf{r} ; \alpha)=\mathbf{P}_{t} \eta(\mathbf{r} ; \alpha)
$$

where $\mathbf{P}_{t}$ also rotates with the particle as will be discussed later.

The above formula for electrostatic interaction can be expanded to magnetostatic interaction conveniently. The overall magnetic field generated by arbitrary field of magnetization $\mathbf{M}(\mathbf{r})$ is given by ${ }^{64}$

$$
\mathbf{H}(\mathbf{r})=\mathbf{H}^{e x}-\int \frac{d^{3} k}{(2 \pi)^{3}}[\mathbf{n} \cdot \tilde{\mathbf{M}}(\mathbf{k})] \mathbf{n} e^{i \mathbf{k} \cdot \mathbf{r}}
$$

where $\tilde{\mathbf{M}}(\mathbf{k})$ is Fourier transform of magnetization distribution function $\mathbf{M}(\mathbf{r})$. The magnetostatic force density is

$$
d \mathbf{F}^{m a g}(\alpha)=\mu_{0}[\mathbf{M}(\mathbf{r} ; \alpha) \cdot \nabla] \mathbf{H}(\mathbf{r}) d V
$$

The corresponding torque density is

$$
d \mathbf{T}^{m a g}(\alpha)=\mu_{0} \mathbf{M}(\mathbf{r} ; \alpha) \times \mathbf{H}(\mathbf{r}) d V+\mu_{0}\left[\mathbf{r}-\mathbf{r}^{c}(\alpha)\right] \times\{[\mathbf{M}(\mathbf{r} ; \alpha) \cdot \nabla] \mathbf{H}(\mathbf{r})\} d V
$$




\subsection{Kinetic equations of the multiphase-liquid colloid systems}

\subsubsection{Kinetic description of particles}

Colloidal particles are assumed rigid (no deformation) in the fluid. At small Reynolds number $(\operatorname{Re}=V L / v$, where $V, L$, and $v$ are characteristic velocity, length, and kinematic viscosity, respectively.), for an isolated particle of arbitrary shape in unbounded fluid the particle center translational velocity $\mathbf{V}^{c}$ and angular velocity $\boldsymbol{\Omega}$ are given by ${ }^{60}$

$$
\begin{aligned}
& V_{i}^{c}(\alpha)=M_{i j}(\alpha) F_{j}(\alpha)+U_{i j}(\alpha) T_{j}(\alpha) \\
& \Omega_{i}(\alpha)=V_{i j}(\alpha) F_{j}(\alpha)+N_{i j}(\alpha) T_{j}(\alpha)
\end{aligned}
$$

where $F_{j}(\alpha)$ and $T_{j}(\alpha)$ are the resultant external force and torque applied to the particle $\alpha$, and $M_{i j}, U_{i j}, V_{i j}, N_{i j}$ are independent mobility matrices that are intrinsic properties of particles (only linked to particle geometries). If there exist three mutually perpendicular symmetry planes for the particle, then the coupling matrices $U_{i j}$ and $V_{i j}$ become zero and the above equations are simplified as ${ }^{60}$

$$
\begin{aligned}
& V_{i}^{c}(t ; \alpha)=M_{i j}(t ; \alpha) F_{j}(t ; \alpha) \\
& \Omega_{i}(t ; \alpha)=N_{i j}(t ; \alpha) T_{j}(t ; \alpha)
\end{aligned}
$$

The $\mathbf{M}$ and $\mathbf{N}$ mobility matrices are attached to particles and rotate by ${ }^{64}$

$$
\begin{aligned}
& M_{i j}(t, \alpha)=Q_{i k}(t, \alpha) Q_{j l}(t, \alpha) M_{k l}\left(t_{0}, \alpha\right) \\
& N_{i j}(t, \alpha)=Q_{i k}(t, \alpha) Q_{j l}(t, \alpha) N_{k l}\left(t_{0}, \alpha\right)
\end{aligned}
$$

where $\mathbf{Q}$ is a rotation matrix and it evolves with time by

$$
Q_{i j}(t+d t ; \alpha)=R_{i k}(t ; \alpha) Q_{k j}(t ; \alpha)
$$

with

$$
R_{i j}(t ; \alpha)=\delta_{i j} \cos \omega+m_{i} m_{j}(1-\cos \omega)-\varepsilon_{i j k} m_{k} \sin \omega
$$


Here $\boldsymbol{\omega} \boldsymbol{\Omega}(t ; \alpha) d t$ is the rotation angle, $\mathbf{m} \boldsymbol{\theta} / \omega$ is the unit vector along the rotation axis, and $\varepsilon_{i j k}$ the permutation symbol. Given the velocity and angular velocity of particles, the field variables are updated by

$$
\eta(\mathbf{r}, t ; \alpha)=\eta\left(\mathbf{r}^{0}, t_{0} ; \alpha\right)
$$

with the current and original position vectors linked by

$$
r_{i}=Q_{i j}(t ; \alpha)\left[r_{j}^{0}-r_{j}^{c}\left(t_{0} ; \alpha\right)\right]+r_{i}^{c}(t ; \alpha)
$$

and

$$
r_{i}^{c}(t+d t ; \alpha)=r_{i}^{c}(t ; \alpha)+V_{i}^{c}(t ; \alpha) d t
$$

In case of spherical particles, the mobility matrices are respectively

$$
\begin{aligned}
& M_{i j}(\alpha)=\frac{\delta_{i j}}{6 \pi \eta a} \\
& N_{i j}(\alpha)=\frac{\delta_{i j}}{8 \pi \eta a^{3}}
\end{aligned}
$$

where $a$ is particle radius. It needs to be noted that the above derivation did not include Stokeslet type hydrodynamic interaction. ${ }^{60,63}$ If necessary, it is viable to employ the Oseen tensor to include Stokeslet type hydrodynamic interaction between close particles. ${ }^{1 \text {, }}$ 63

\subsubsection{Kinetic description of liquid phase separation}

Since Cahn and Hilliard published their milestone work about spinodal decomposition, ${ }^{80}$ the diffuse interface method has been widely accepted in describing phase evolution processes. In our DIFA model, the concentration field variables of liquid phases evolve in time following the Cahn-Hilliard equation:

$$
\frac{\partial c_{\alpha}}{\partial t}=\nabla \cdot\left(M_{\alpha} \nabla \frac{\delta F}{\delta c_{\alpha}}\right)
$$

where $M_{\alpha}$ is chemical mobility, and 


$$
\frac{\delta F}{\delta c_{\alpha}}=\frac{\partial f}{\partial c_{\alpha}}-\kappa_{\alpha} \Delta c_{\alpha}
$$

is identified as the chemical potential $\mu_{\alpha}$. Within the framework of Cahn-Hilliard equation, the two-phase fluid morphology evolves through nonlinear diffusion, thus it is assumed that colloids are dispersed in otherwise quiescent viscous binary liquids undergoing quasistatic motion where Reynolds number $R e$ and Péclet number ( $P e=V L / D$, where $D$ is the liquid diffusivity.) are very small. Colloidal particles represented by diffuse interface fields $\left\{\eta_{\beta}\right\}$ evolve via rigid-body motions subjected to various forces including interfacial tension, Laplace pressure, short-range and long-range interactions as previously stated.

\section{Spectral method to solve Cahn-Hilliard equation}

By using Fourier Transform, Cahn-Hilliard equation becomes

$$
\frac{\partial \tilde{c}_{i}}{\partial t}=-M_{i} k^{2}\left(F T\left[\frac{\partial f}{\partial c_{i}}\right]+\beta_{i} k^{2} \tilde{c}_{i}\right)
$$

The above equation is then solved in k-space, and one can use a semi-implicit spectral algorithm to improve its convergence efficiency. ${ }^{81,82}$ At the $(n+1)$ th time step, the concentration of each fluid is updated from the $\mathrm{n}$ th time step in $\mathrm{k}$-space by

$$
\tilde{c}_{i}^{(n+1)}=\frac{\tilde{c}_{i}^{(n)}-M_{i} k^{2} \widetilde{f}^{\prime}\left(c_{i}\right)^{(n)} d t}{1+M_{i} \beta_{i} k^{4} d t} \quad(i=1,2)
$$

where $\widetilde{f^{\prime}}\left(c_{i}\right)$ stands for Fourier Transform of $\frac{\partial f}{\partial c_{i}}$. Concentration fields in the $(\mathrm{n}+1) t h$ time step are obtained by inverse Fourier Transform

$$
c_{i}^{(n+1)}=\operatorname{real}\left(F T^{-1}\left[\tilde{c}_{i}^{(n+1)}\right]\right) \quad(i=1,2)
$$

The semi-implicit algorithm has proven to be of significantly improved efficiency than the explicit finite difference scheme. The time step can be effectively 2 or 3 orders of magnitude larger than the explicit finite difference scheme. ${ }^{81,82}$ 


\section{Correction to drift of mass caused by long time diffusion simulation}

It is worth noting that accumulated round-off errors during numerical solution of diffusion equation over long time (large number of iterations) may generate nonnegligible composition drift. Even for the semi-implicit spectral method with improved efficiency, mass drift is still not resolved completely. In order to avoid error accumulation and conserve mass, in this thesis a very minor correction is made in the simulations after each time increment: ${ }^{37}$

$$
c_{i} \leftarrow c_{i} C_{i}^{0} / C_{i}
$$

where

$$
C_{i}^{0}=\int c_{i}(\mathbf{r}, t=0) d V
$$

is the total initial mass of each liquid phase, and $C_{i}=\int c_{i}(\mathbf{r}, t) d V$. While such a correction is negligibly small in each time increment, it is necessary for simulations over a large number of iteration steps to avoid accumulation of concentration drift.

\subsubsection{Model verification and capability test (bijel formation)}

\section{Bijel formation:}

The formation of binary interface jammed emulsion gels (bijel) has first been computational predicted. Stratfard et al simulated the process that leads to the bijel by Lattice Boltzmann method, combined with a Cahn-Hilliard model for binary solution. ${ }^{21}$ Here the spinodal decomposition process of a binary solution that contains colloidal particles is simulate by DIFA. The particles are predefined to be elliptical and have equal affinity to each product liquid phase. In other words, the contact angle is $90^{\circ}$ at the threephase contact line. (In two dimensional simulations, it is contact spot instead of contact line). 
Figure 2.5 shows the evolution sequence. Figure 2.5a-c exhibit regular coarsening behavior in phase separation. Most particles are adsorbed at liquid interfaces with their long axes aligned with the liquid interfaces to minimize interfacial energy. At the initial stages, the particles are apart from each other with gap distance such that no jamming exists. As a result, the liquid interfaces involve with their curvature not affected by isolated adsorbed particles (as will be discussed in detail in next chapter). Meanwhile, some particles are bridged by two adjacent fluid droplets, which may to some extent prevent the droplets from draining. ${ }^{83}$ Such phenomena, have also been observed experimentally during bijels formation. ${ }^{17}$

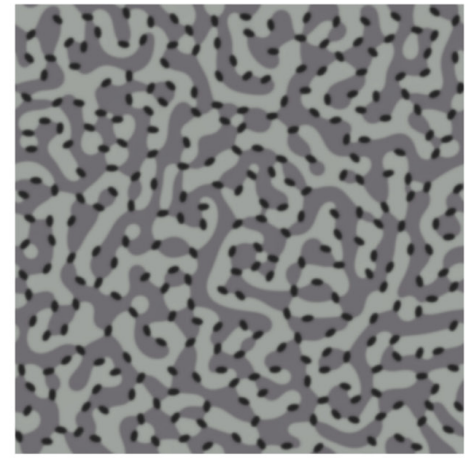

(a) $t^{*}=4,000$

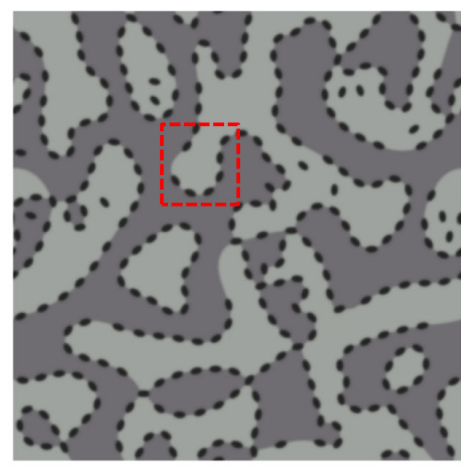

(d) $t^{*}=60,000$

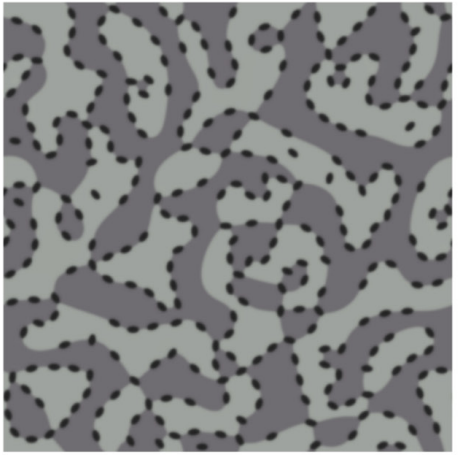

(b) $t^{*}=20,000$

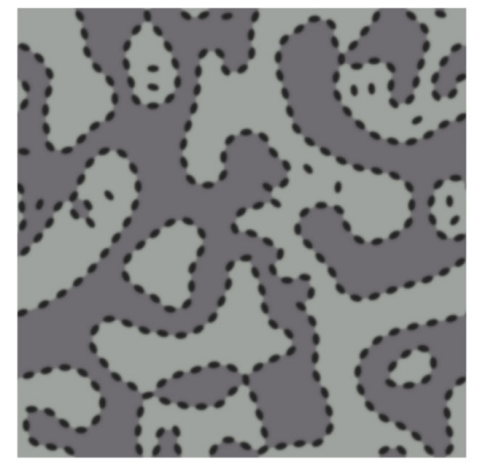

(e) $t^{*}=200,000$

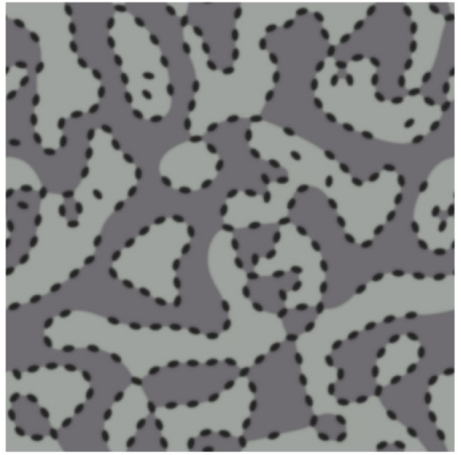

(c) $t^{*}=40,000$

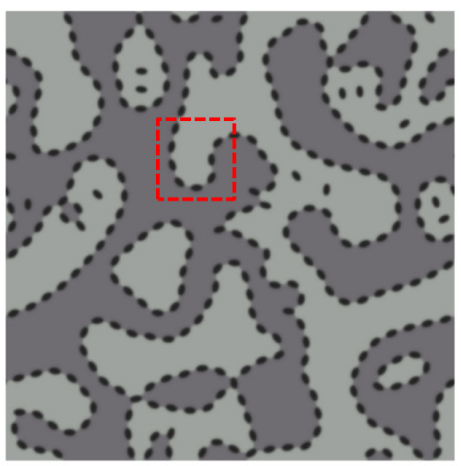

(f) $t^{*}=400,000$

Figure 2.5 Simulated jamming of ellipse-shaped particles at liquid interfaces during late stage of spinodal decomposition. The configuration in (f) is almost unchanged from (e). 
From Figure $2.5 \mathrm{c}$, it is seen that some droplets are gradually enclosed by a monolayer of particles which stabilized the droplet and prevent it from further shrinkage. In Figure $2.5 \mathrm{~d}$, there are still some naked liquid interfaces, e.g., the region shown in the highlighted box. In Figure 2.5e, almost all liquid interfaces are loaded with particles, which makes the structure almost frozen. As Figure $2.5 \mathrm{f}$ is apparently the same as Figure 2.5e, except for some rotational motion of particles (compare the particles orientation outside the bottom right corner of the highlight box with that in Figure 2.5e). The simulation shows that after jamming, it is possible that the particles are squeezed and distorted off from alignment with the tangential direction of its attached liquid interface.

Figure 2.6 is the distribution of internal pressure during colloidal jamming corresponding to Figure 2.5. It is clear that during jamming, the magnitude of Laplace pressure is decreasing. The surface pressure is most significant beside naked liquid interfaces with curvature. After fully jammed, the Laplace pressure is virtually absent in the liquid. From the viewpoint of Young-Laplace equation, the radius of curvature is increasing to infinity, which means the liquid interfaces are almost flat.

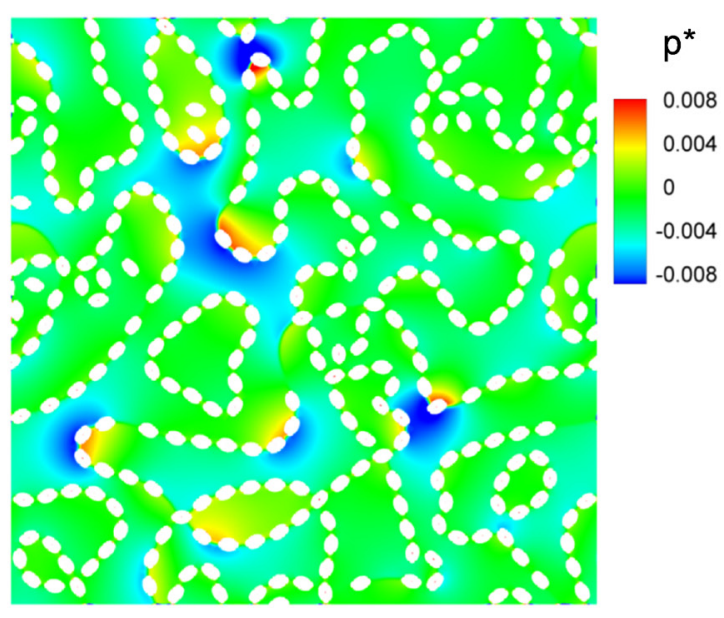

(a) $t^{*}=60,000$

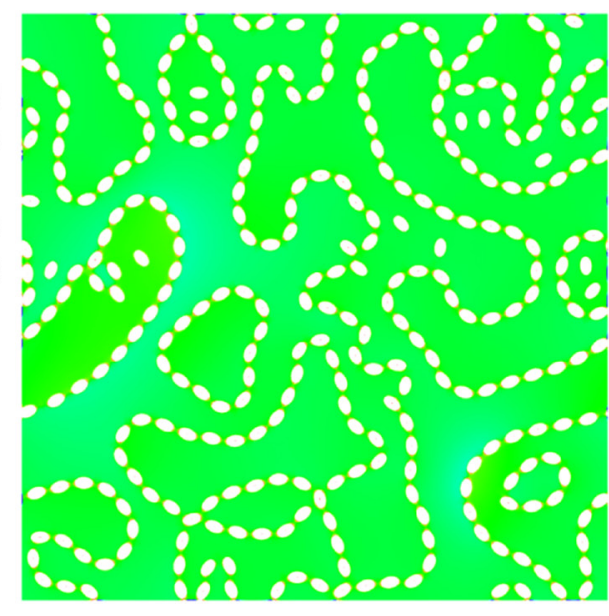

(b) $t^{*}=400,000$

Figure 2.6 Surface pressure distribution during the late stages of spinodal decomposition. (a) is corresponding to Figure $2.5 \mathrm{~d}$, and (b) to Figure 2.5e. The white color stands for particles. 


\subsection{Phase field model for dipolar domains in ferroic materials}

In ferroic materials with dipolar domains, dipole-dipole interaction takes significant roles as well. Differences are that in ferroic system the dipoles are confined with crystalline cells and thus do not undergo rigid body motion as colloidal particles do. In addition, isolated dipolar colloidal particles in monophase carrier liquid are free to rotate,

whereas polarization in ferroelectric crystals and magnetization in ferromagnetic materials have strong orientation preference associated to crystalline structure. Despite of distinctness, the common dipole-dipole interaction links these material systems together and exhibits interesting phenomena.

\subsubsection{Ferroelectric system}

Ferroelectric domains can be well modeled by phase field method. The total system free energy for a single crystal ferroelectric system is given by

$$
F=\int d^{3} r\left[f(\mathbf{P})+\frac{1}{2} \beta_{i j k l} \frac{\partial P_{i}}{\partial r_{j}} \frac{\partial P_{k}}{\partial r_{l}}-E_{k}^{0} P_{k}\right]+\frac{1}{2} \int \frac{d^{3} k}{(2 \pi)^{3}}\left[\frac{n_{i} n_{j}}{\varepsilon_{0}} \tilde{P}_{i} \tilde{P}_{j}^{*}+K_{i j k l} \tilde{\varepsilon}_{i j}^{0} \tilde{\varepsilon}_{k l}^{0^{*}}\right]
$$

where summation convention over repeated indices is implied. $\beta_{\mathrm{ijkl}}$ is gradient coefficient tensor. The function $f(\mathbf{P})$ is the non-equilibrium local bulk free energy density that defines the thermodynamic properties of stress-free homogeneous ferroelectric state, and is formulated by Landau-Ginzburg-Devonshire theory:

$$
\begin{aligned}
f(\mathbf{P}) & =\alpha_{1}\left(P_{1}^{2}+P_{2}^{2}+P_{3}^{2}\right)+\alpha_{11}\left(P_{1}^{4}+P_{2}^{4}+P_{3}^{4}\right)+\alpha_{12}\left(P_{1}^{2} P_{2}^{2}+P_{2}^{2} P_{3}^{2}+P_{3}^{2} P_{1}^{2}\right) \\
& +\alpha_{111}\left(P_{1}^{6}+P_{2}^{6}+P_{3}^{6}\right)+\alpha_{112}\left[P_{1}^{4}\left(P_{2}^{2}+P_{3}^{2}\right)+P_{2}^{4}\left(P_{3}^{2}+P_{1}^{2}\right)+P_{3}^{4}\left(P_{1}^{2}+P_{2}^{2}\right)\right] \\
& +\alpha_{123} P_{1}^{2} P_{2}^{2} P_{3}^{2}
\end{aligned}
$$

where the expansion coefficients $\alpha_{\mathrm{i}}, \alpha_{\mathrm{ij}}$, and $\alpha_{\mathrm{ijk}}$ are determined from experimental data for specific material system. The second term is the gradient energy across ferroelectric 
domain walls. The third term designates energy of external electric field applied. The gradient term in Eq. (2.44) characterizes the energy contribution associated with polarization change across domain walls. The k-space integral in Eq. (2.44) gives the electrostatic energy of distributed polarization field $\mathbf{P}(\mathbf{r})$, which is the dipole-dipole interaction energy, and the elastostatic energy of electrostrictive strain distribution,

$$
\varepsilon_{i j}^{0}(\mathbf{r})=Q_{i j k l} P_{k}(\mathbf{r}) P_{l}(\mathbf{r})
$$

where $Q_{\mathrm{ijkl}}$ is electrostriction coefficient tensor, $\mathrm{K}_{\mathrm{ijk}}=\mathrm{C}_{\mathrm{ijk} l}-\mathrm{n}_{\mathrm{m}} \mathrm{C}_{\mathrm{ijmn}} \Omega_{\mathrm{np}} \mathrm{C}_{\mathrm{klpq}} \mathrm{n}_{\mathrm{q}}$, $\Omega_{\mathrm{ik}}=\left(\mathrm{C}_{\mathrm{ijk} \mid} \mathrm{n}_{\mathrm{j}} \mathrm{n}_{\mathrm{l}}\right)^{-1}, \mathrm{C}_{\mathrm{ijk} \mathrm{l}}$ is elastic modulus tensor, and $\mathbf{n}=\mathbf{k} / k$. The functions $\tilde{\mathbf{P}}(\mathbf{k})$ and $\tilde{\boldsymbol{\varepsilon}} \mathbf{k}\left(\right.$ ) are the Fourier transform of the fields $\mathbf{P}(\mathbf{r})$ and $\varepsilon^{0}(\mathbf{r})$, respectively, and the superscript asterisk * indicates complex conjugate.

The kinetics of ferroelectric domains (polarization) obeys the time dependent Ginzburg-Landau equation, which is

$$
\frac{\partial P_{i}(\mathbf{r}, t)}{\partial t}=-L_{i j} \frac{\delta F}{\delta P_{j}(\mathbf{r}, t)}+\xi_{i}(\mathbf{r}, t)
$$

where $L_{i j}$ is kinetic coefficient and the last term is Gauss distributed Langevin noise representing thermal fluctuation.

\subsubsection{Ferromagnetic system}

Magnetic domains can be studied by micromagnetic modeling, ${ }^{84-86}$ which in a broader sense, can also called DIFA. Magnetization vector M(r) has a nearly constant magnitude $\mathrm{M}_{\mathrm{s}}$ (saturation magnetization) but its direction changes from one domain to another and may evolve with time. Given the constant magnetization vector length, the unit directional vector field $\mathbf{m}(\mathbf{r})=\mathbf{M}(\mathbf{r}) / \mathrm{M}_{\mathrm{s}}$ is conveniently used to describe the magnetic domain structure. The total system free energy is a sum of magnetocrystalline energy, exchange energy, magnetostatic energy, and external magnetic energy: 


$$
\begin{aligned}
F= & \int K_{1}\left\{1-[\mathbf{t}(\mathbf{r}) \cdot \mathbf{m}(\mathbf{r})]^{2}\right\} d^{3} r+A \int|\operatorname{grad} \mathbf{m}(\mathbf{r})|^{2} d^{3} r+\frac{\mu_{0} M_{s}^{2}}{2} f \frac{d^{3} k}{(2 \pi)^{3}}|\mathbf{n} \cdot \tilde{\mathbf{m}}(\mathbf{k})|^{2} \\
& -\mu_{0} M_{s}^{2} \int \mathbf{h}^{\mathrm{ext}} \cdot \mathbf{m}(\mathbf{r}) d^{3} r
\end{aligned}
$$

where $K_{1}$ is the magnetocrystalline anisotropy constant, the spatially distributed vector field $\mathbf{t}(\mathbf{r})$ characterizes the local orientation of tetragonal axis, A is the exchange stiffness constant, $\mu_{0}$ the vacuum permeability, $\mathbf{h}^{\text {ext }}$ the normalized external magnetic field $\mathbf{h}^{\text {ext }}=\mathbf{H}^{\text {ext }} / M_{s}, f$ is evaluated as a principal value excluding the point $\mathbf{k}=\mathbf{0}$, $\tilde{\mathbf{m}}(\mathbf{k})=\int \mathbf{m}(\mathbf{r}) e^{-i \mathbf{k} \cdot \mathbf{r}} d^{3} r$, and $\mathbf{n}=\mathbf{k} / k$ is a unit directional vector in reciprocal space. The elastic energy is neglected since it takes a minor role on affecting magnetic domain evolution in most cases, and it can be incorporated if necessary, just similar to the elastostatic energy in ferroelectric materials which is presented later. The evolution of magnetization vector field is described by the Landau-Lifshitz-Gilbert equation:

$$
\frac{\partial \mathbf{m}(\mathbf{r}, t)}{\partial t}=-\frac{\gamma}{\mu_{0} M_{s}\left(1+\alpha^{2}\right)}\left[\mathbf{m} \times \frac{\delta F}{\delta \mathbf{m}}-\alpha \mathbf{m} \times\left(\mathbf{m} \times \frac{\delta F}{\delta \mathbf{m}}\right)\right]
$$

where $\gamma$ is the gyromagnetic ratio, and $\alpha$ the damping parameter. 


\section{Chapter 3. Colloids at liquid interfaces: The role of Laplace pressure}

\subsection{Isolated particles at liquid interfaces}

Engineered colloidal particles dispersed in multi-phase liquids offer enormous application opportunities, while also pose great challenges to a complete scientific understanding due to the complexities of interaction forces and microstructural evolutions. In order to understand the microstructures and properties of such multi-liquid-phase

colloidal systems, the behaviors of colloidal particles at liquid interfaces are one of the most important issues that must be investigated. Realistic computer modeling and simulation is highly desired for such a study, which not only complements experimental studies by providing quantitative details but can also artificially switch on/off various interaction forces at will, enabling researchers to focus on individual interactions separately as well as any combination of them, which are usually difficult or practically impossible for experiments. The purpose of work of the following section is to present such a computer modeling and simulation study of shape-anisotropic particles at curved fluid interfaces under the influences of interfacial tension and Laplace pressure (the two distinct contributions to capillary forces) as well as the resultant collective behaviors of colloidal particles and multi-phase fluid.

\section{Modeling details}

The free energy function for a two-phase liquid colloid system is described in Eq. (2.1)-(2.2). Other modeling methods have been described in Chapter 2. The resultant force and torque acting on particle $\beta$ are given by 


$$
\begin{aligned}
& \mathbf{F}(\beta)=\int_{V}\left[\xi d \mathbf{F}^{\mathrm{LP}}(\mathbf{r}, \beta)+d \mathbf{F}^{\mathrm{IT}}(\mathbf{r}, \beta)+d \mathbf{F}^{\mathrm{SR}}(\mathbf{r}, \beta)\right]+{ }^{\mathrm{F}}(\beta) \\
& \mathbf{T} \mathbf{(} \beta) \mathbf{r}=\int_{V}\left[\xi^{\mathrm{c}}(\boldsymbol{B} \beta)\right] \mathbf{x}\left[d^{\mathrm{LP}}(\mathbf{F}, \beta) \mathbf{r}+d^{\mathrm{IT}}(\mathbf{F}, \beta) \mathbf{r}+d^{\mathrm{SR}}(, \beta)\right]+{ }^{\mathrm{T}}(\beta)
\end{aligned}
$$

where $\mathbf{r}^{\mathrm{c}}(\beta)$ is the center of mass of particle $\beta, \xi^{\mathrm{F}}(\beta)$ and $\xi^{\mathrm{T}}(\beta)$ are Gaussiandistributed Langevin noises representing Brownian motion forces. Here we set $<\xi \xi(\beta)^{2}>=<{ }^{F}(\beta)^{2}>\bar{r}^{2}$, where $\bar{r}$ is mean radius of particle. As has been stated in Section 2.2, the thermal fluctuation energy is negligible compared with the interfacial energy barrier to remove a particle from liquid interface, and Brownian force is also very small compared with capillary forces.

A semi-implicit spectral method (see Section 2.3.2) is employed to solve the CahnHilliard equation, which offers significantly improved efficiency and accuracy comparing to the explicit Euler scheme ${ }^{81,82}$. The time increment is set to $\Delta t=0.5 l^{2} /(\Delta H M)$, where $l$ is computational grid size, $\Delta H$ is the enthalpy of mixing of the binary solution, and $M_{\alpha}=M$. Since simulation of long time diffusion tends to cause drifting of concentration fields, in our simulation an instant concentration field correction algorithm is employed to guarantee mass conservation ${ }^{37}$ (see Section 2.3.2). All parameters are normalized and the normalization is listed in Table 3.1.

In the simulations, the parameters $A$ and $\chi$ are set to $1 / 12$ and 1 , respectively, and $\lambda_{A}=\lambda_{B}=1.0, \kappa_{A}=\kappa_{B}=0.6$, which give approximately $3 l$ thickness of all diffuse interfaces between $10 \%$ and $90 \%$ of full equilibrium value, and interface energy (normalized by $\Delta H l$ ) $\gamma_{A B}=0.254$ for liquid-liquid interface, $\gamma_{A S}=\gamma_{B S}=0.174$ for interfaces between the two liquid phases and particles. The contact angle of liquid interface at the particle surface is thus $\theta=90^{\circ}$. Periodic boundary condition is employed for all simulations. While the DIFA model can be applied to three dimensions, computer simulations reported in this work are performed in two dimensions. 
Table 3.1

Normalization of parameters used in simulations in Chapter 3.

\begin{tabular}{|c|c|c|c|c|}
\hline Parameters & $\begin{array}{c}\text { Characteristic } \\
\text { unit }\end{array}$ & $\begin{array}{c}\text { Reduced } \\
\text { value used }\end{array}$ & SI unit & $\begin{array}{c}\text { Magnitude of } \\
\text { characteristic } \\
\text { unit } \\
\end{array}$ \\
\hline Grid size & $l_{0}$ & 1 & {$[\mathrm{~m}]$} & $10^{-7}$ \\
\hline $\begin{array}{r}\text { Fluid } \\
\text { chemical } \\
\text { mobility }\end{array}$ & $M_{0}$ & 1 & {$[\mathrm{~N}]^{-1}[\mathrm{~m}]^{4}[\mathrm{~s}]^{-1}$} & $10^{-15}$ \\
\hline $\begin{array}{l}\quad \text { Particle } \\
\text { translation } \\
\text { mobility }\end{array}$ & $M_{0} / l_{0}^{3}$ & $\sim 0.01$ & {$[\mathrm{~N}]^{-1}[\mathrm{~m}][\mathrm{s}]^{-1}$} & $10^{6}$ \\
\hline $\begin{array}{l}\text { Rotation } \\
\text { mobility }\end{array}$ & $N_{0}=M_{0} / l_{0}^{5}$ & $\sim 0.0001$ & {$[\mathrm{~N}]^{-1}[\mathrm{~m}]^{-2}[\mathrm{~s}]^{-1}$} & $10^{20}$ \\
\hline Time step & $l_{0}^{2}\left(M_{0} \Delta H\right)^{-1}$ & $\sim 0.5$ & [s] & $10^{-4}$ \\
\hline $\begin{array}{l}\text { Interfacial } \\
\text { tension }\end{array}$ & $\gamma_{0}=\Delta H l_{0}$ & $\sim 0.25$ & {$[\mathrm{~J}][\mathrm{m}]^{-2}$} & $10^{-3}$ \\
\hline $\begin{array}{l}\text { Rigid body } \\
\text { coeff. }\end{array}$ & $\gamma_{0}$ & 25 & {$[\mathrm{~Pa}]$} & $10^{-2}$ \\
\hline $\begin{array}{l}\text { Surface force } \\
\text { coeff. }\end{array}$ & $\kappa_{0}=\gamma_{0} / l_{0}^{2}$ & $\sim 9.13$ & {$[\mathrm{~N}][\mathrm{m}]^{-3}$} & $10^{11}$ \\
\hline $\begin{array}{l}\text { Chemical } \\
\text { free energy } \\
\text { parameter }(\mathrm{A})\end{array}$ & $A_{0}=\Delta H$ & $1 / 12$ & {$[\mathrm{~J}][\mathrm{m}]^{-3}$} & $10^{4}$ \\
\hline $\begin{array}{l}\quad \text { Gradient } \\
\text { coeff of order } \\
\text { parameters }\end{array}$ & $\beta_{0}=\gamma_{0} l_{0}$ & $\sim 1$ & {$[\mathrm{~J}][\mathrm{m}]^{-1}$} & $10^{-10}$ \\
\hline $\begin{array}{r}\text { Langevin } \\
\text { noise(Force) }\end{array}$ & $\xi_{0}=\gamma_{0} l_{0}$ & 0.0001 & {$[\mathrm{~N}]$} & $10^{-11}$ \\
\hline
\end{tabular}

\section{Simulation results}

It is a fundamental question that how a single particle adsorbed at a fluid interface affects the morphology of the interface. At the zone where three phases (liquid-liquidsolid particle) meet together, the contact angle must satisfy Young's equation. Meanwhile, 
the particle is subject to interfacial tension as well as Laplace pressure. For a spherical particle, it is well known that at equilibrium state, the Laplace pressure on the particle exactly balances the interfacial tension, and the contact angle can be satisfied with appropriate position of the particle. ${ }^{36}$ By taking this phenomenon as a standard, the coefficient $\kappa_{P}$ in Eq. (2.12) can be calibrated and $\kappa_{P} \approx 1$. This coefficient is approximately unity for along any radial direction $\mathbf{n}$ the value of the integration $\int \nabla \tilde{\eta} d r=1$.

For nonspherical particles, we choose three representative shapes of ellipse, polygon and star, respectively. The relaxation processes of these shape particles are simulated and shown in Figure 3.1. In Figure 3.1a, the elliptical particle finally lays its long axis aligned with the tangential direction of liquid interface so as to reduce the interfacial energy to the largest degree. In Figure 3.1b, the pentagon shaped particle rotates into its equilibrium by taking two no-neighboring vertices docked at the liquid interfaces, which is also corresponding to the minimum of interfacial energy. In Figure 3.1c, a star shaped particle adjusts its position such that the non-neighboring vertices pinned at the liquid interface. In all three cases, it is found that the final liquid interfaces are maintained to be spherical. In other words, the irregularly shaped particles do not either disturb the curvature of their attached liquid interfaces. For smooth shaped particles, like ellipse, the particle may adjust the contact angle by adjusting its position that intersects the vesicle surface (if we ignore the small volume change of the vesicle due to the part immersion of the particle). For the pentagon and star with corners, however, there is great flexibility for contact angle at the arrises of surfaces. Such an effect is believed to be responsible for the hysteresis of apparent contact angle on rough surfaces ${ }^{69,70}$. 
(a)
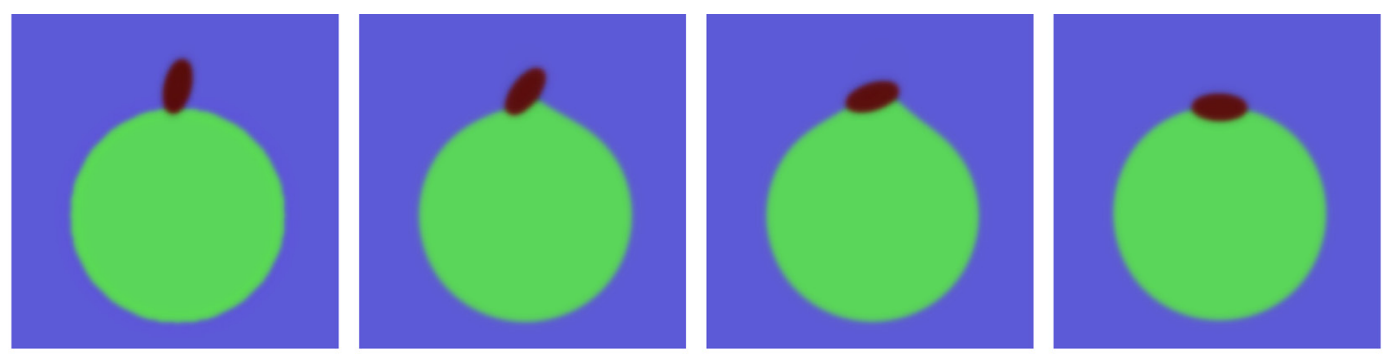

(b)
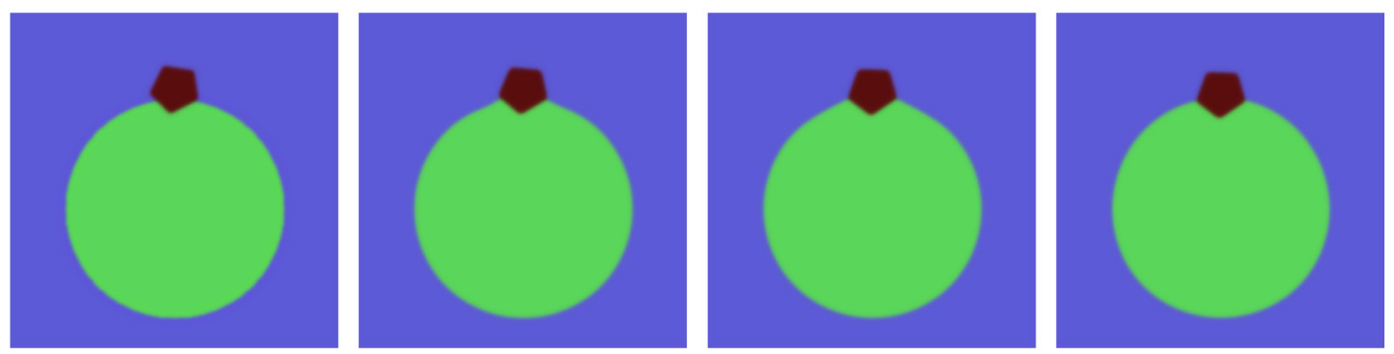

(c)
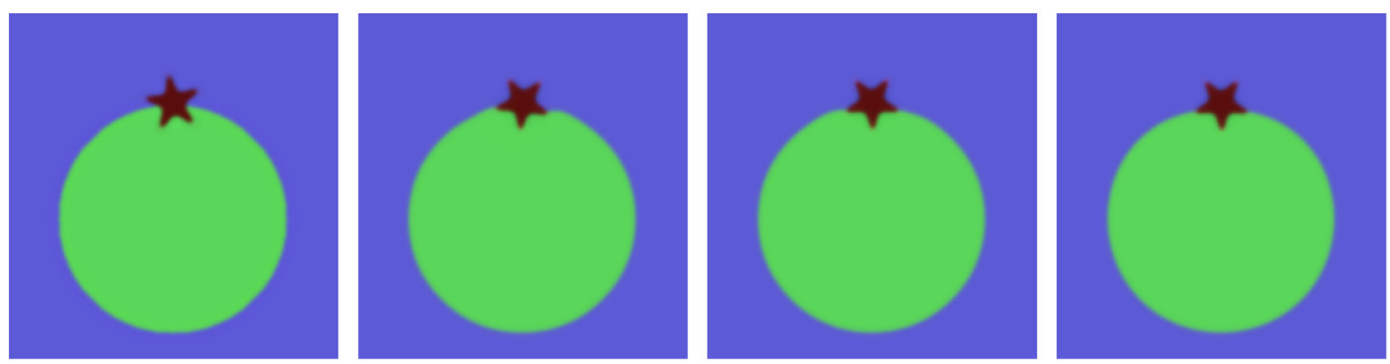

Figure 3.1 Relaxation of various shaped particles at the surface of a vesicle. (The "halo" fields of particles are not shown.) (1) Ellipse (2) Pentagon (3) Star (The different colors represent liquid phases and solid particles. Same for below)

Although the final state of Figure 3.1c appears to be energy minimized state, it is not necessarily the only possible state. Figure 3.2 gives the relaxation of star particle with a different initial configuration. It turns out that at final equilibrium, the two neighboring vertices are pinned at the liquid interface, without disturbing the curvature of the spherically curved interface as well. It has to be noted that the situation will be different in three dimensional cases. In 3D case, nonspherical particles typically deform the liquid interface and make it locally undulated since the contact angle has to be satisfied along 
the entire contact line, as has been theoretically investigated ${ }^{87}$ and experimentally observed $^{88}$.
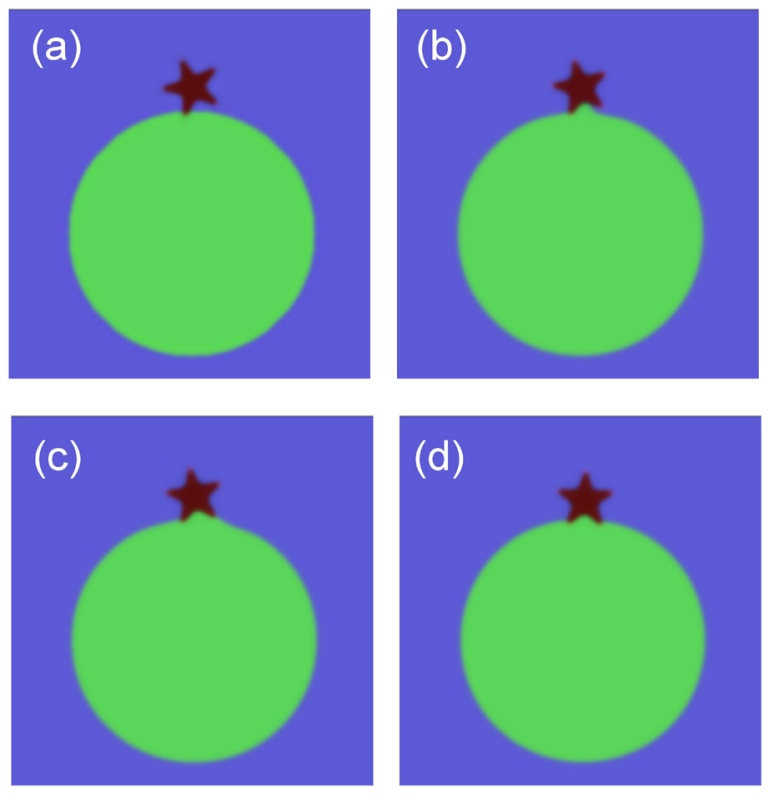

Figure 3.2 Star shaped particle may choose to relax at a metastable state instead of the energy minimum state, due to the flexibility for contact angle at the vertices

The above results indicate that without external force, an isolated particle adsorbed at fluid interface does not disturb the interface, since the Laplace pressure acting on it exactly balances the interfacial tension. This is true in two dimensional cases, for sphere, ellipse, polygons, and even particles with rugged surface (like the star), since the contact angle requirement is easily satisfied at the two contact spots. This means that two or more separated particles without external force or interparticle force can also stay at the interface without lateral capillary interaction. 


\subsection{Colloid jamming at liquid interfaces}

Capillary forces on isolated particles at liquid interface without interparticle force are self-balanced, while if particles are jammed together, interparticle forces may break the balance and thus alter the interface morphology. As a result, the Laplace pressure does not exactly balance interfacial tension and the net capillary force must be balanced by interparticle forces (such as steric repulsion) for equilibrium. For simplicity, we first consider spherical particles (in 2D they are actually cylindrical with infinite length) jammed at the surface of a spherical droplet. The actual Laplace pressure across the jammed interface can be detected by a coexisting free droplet at equilibrium. Figure 3.3 shows a simulated process of diffusional equilibrium between a free droplet and a droplet coated with spherical particles. In our model, the interfacial tension in the gap between particles is identical to that of free interface $\gamma$. In two dimensional cases, the relationship between interparticle force $F$ and the radius of curvature of the liquid interface as well as the contact angle $\theta$ can be derived analytically. For configuration shown in Figure 3.4, assuming there are $N$ particles uniformly spaced at the droplet surface, the interparticle force determined by static equilibrium is given by (see supplementary material in 3.6.2)

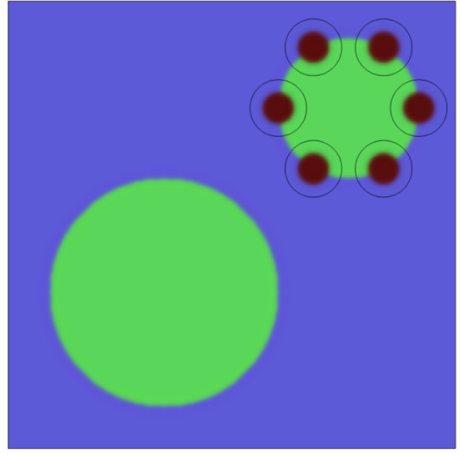

$t^{*}=0$

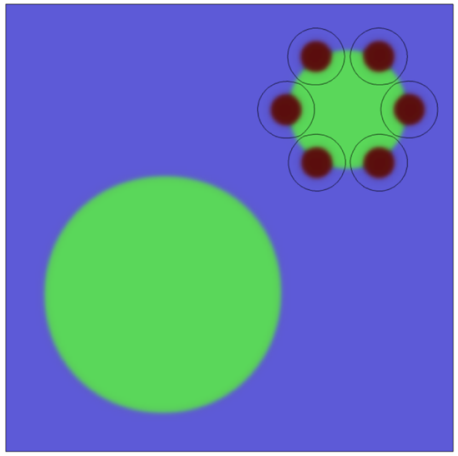

$t^{*}=1 \times 10^{5}$

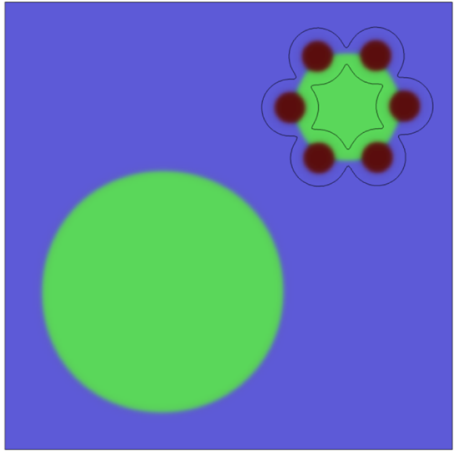

$t^{*}=1 \times 10^{6}$

Figure 3.3 Diffusional equilibrium between a Pickering emulsion droplet and a free droplet. The solid lines around the particles denote the periphery of the "halo" field of particles (coating layers), while the merge of the coating layers indicates steric repulsion. 


$$
F=\frac{\gamma}{\sin \omega}\left[\sin (\theta+\alpha)-\frac{r}{l} \sin \alpha\right]
$$

where $\omega=\pi / N$ and

$$
\alpha=-\omega+\sin ^{-1}\left(\frac{R \sin \omega}{L}\right)+\sin ^{-1}\left(\frac{l \sin \theta}{L}\right)
$$

with $L=\overline{A C}=\sqrt{r^{2}+l^{2}-2 r l \cos \theta}$ (by the law of cosines). If one conducts a virtual experiment, the actual radius of curvature of the droplet surface coated with jamming particles can be obtained simply by measuring the radius of the coexisting free droplet, for at equilibrium the pressure in the two droplets equals $p=\gamma / l$. Since the interparticle distance is $2 R \sin \omega$, the interparticle force-distance relationship is then obtained. On the other hand, the interparticle short range repulsive force, determined by the predefined "halo" field, can be calibrated by Eq. (2.11).

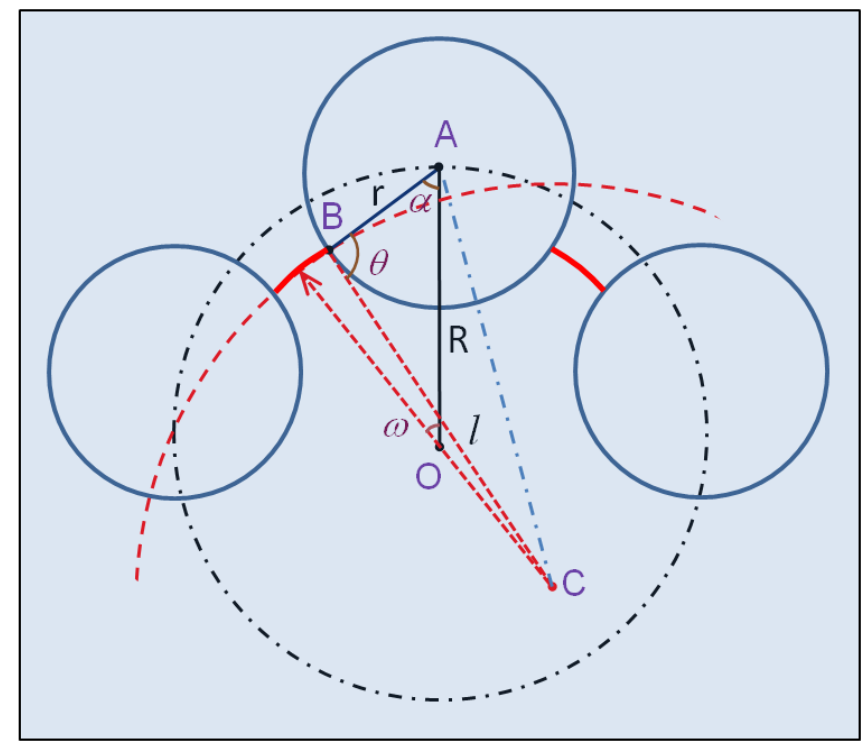

Figure 3.4 Schematic of liquid interface morphology of an emulsion droplet adsorbed with particles. $r=\overline{A B}$ is the radius of particles. The solid red line indicates the position of liquid interface with radius of curvature $l=\overline{C B}$ different from the apparent radius of curvature of the particle contour $R=\overline{O A}$. 


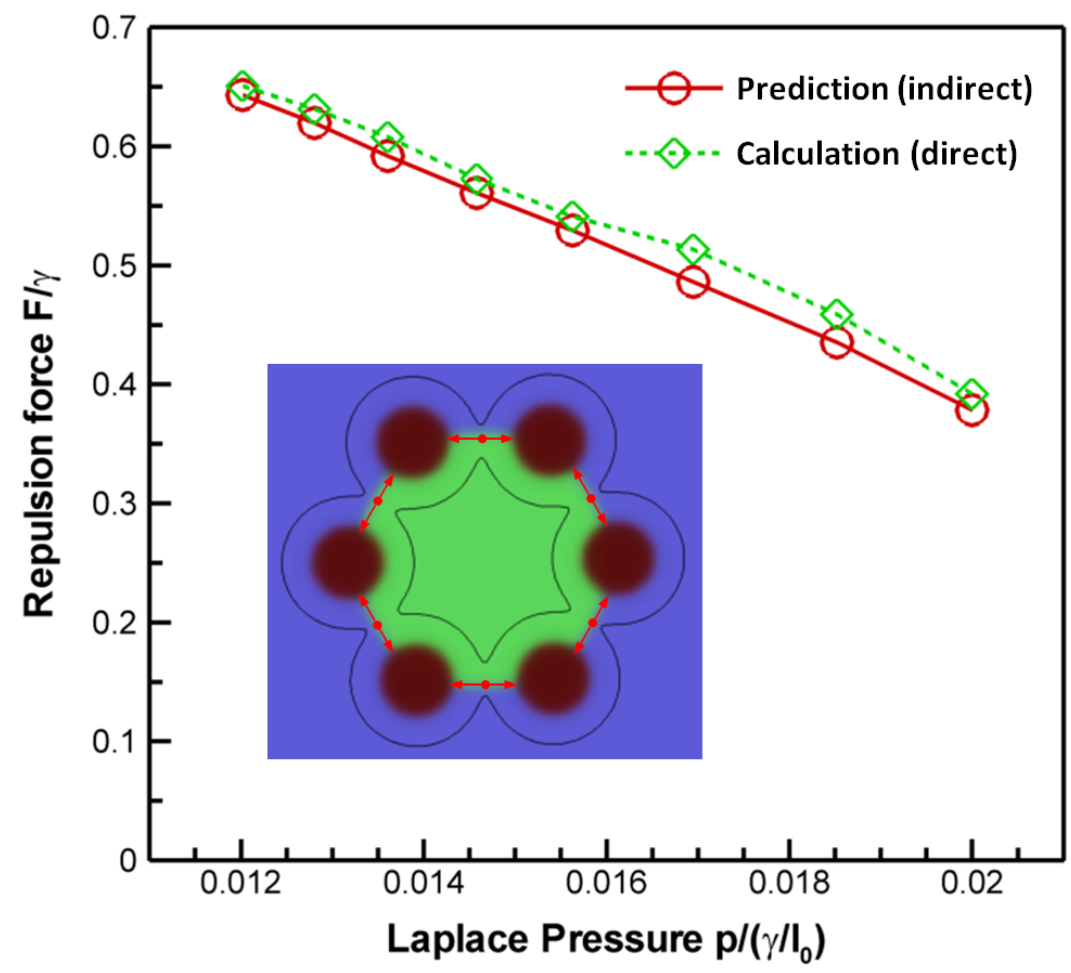

Figure 3.5 Simulation results show interparticle force of a colloidal shell as a function of Laplace pressure.

Figure 3.5 shows a quantitative comparison between interparticle forces predicted by Eq. (3.2) and those calculated by direct integration of Eq. (2.11) for a series of coexisting free droplets of different radii. Good agreement is obtained which demonstrates the accuracy of our DIFA model on capturing the capillary forces and contact angle. This virtual experiment also offers an indirect approach to measure the in situ short-range interparticle force-separation relationship. It has to be noted that in 3D cases the problem should be more complicated. Distinctions are that there are two principal radii of curvature for a 3D liquid surface, and moreover on a 3D spherical surface particles cannot be uniformly distributed while maintaining the perfect symmetry. ${ }^{89}$ The unavoidable imperfections of the particle array due to surface curvature, just like a soccer ball cannot be covered purely by hexagons, makes the analytic geometry analysis difficult. However, similar trend for 3D cases is expected, i.e., when the radius of the 
stable coexisting free droplet is bigger than the apparent curvature of the colloidal shell, the effective surface tension $\bar{\gamma}$ of the colloidal shell is decreased (Here we define the effective surface tension $\bar{\gamma}=\Delta p \cdot R=\gamma R / l$ by Young-Laplace equation and the apparent radius of curvature.), which indicates that the repulsive interparticle forces exist to partly counteract the interfacial tension of liquid interfaces.

\subsection{Mechanical stability and collapse of monolayer Pickering emulsions}

On deriving Eq. (3.2)-(3.3) (cf. Figure 3.4) it is assumed that the Laplace pressure is positive thus the curvature of droplet interface is positive. A natural question is that whether the analytic Eq. (3.2) still holds if the Laplace pressure is negative. Following the above analytic geometry analysis, no doubt that a similar solution can be given (see supplementary material in 3.6.2). However, as will be shown in simulation below, the analytic solution turns out to be meaningless. In experiments Datta et $\mathrm{al}^{90}$ set the negative pressure by adding a fixed amount of unsaturated continuous phase. In this simulation, in order to produce stable negative Laplace pressure, initially we set that the colloidal shell containing phase A dispersed in phase B is encompassed by phase A (see Figure 3.6). After diffusional equilibrium, simulation results show that negative Laplace pressure leads to buckling and collapse of the colloidal shell, even initially the colloidal particles are uniformly distributed along the droplet surface and the particles are identically spherical. Under negative pressure, both Laplace pressure and interface tension acted on a particle pull the particle inward to shrink the droplet, which causes mechanical instability of the colloidal shell. The instability is shown to be independent of particle shape, as is shown in Figure 3.7, where a mixture of three different shapes (square, ellipse, circle) of particles is used. This phenomenon is analogous to the well-known crenation of red blood cells due to hypertonic osmosis. ${ }^{91}$ Crumpling and buckling of nanoparticles coated droplets under negative Laplace pressure have actually been experimentally observed. ${ }^{90,}$ 
92 In our simulation, as long as the Laplace pressure is negative, the monolayer colloidal shell always buckles and shrinks, thus the critical pressure that leads to buckling is 0 . Nevertheless, in experiments if the liquid interface is filled with jammed multilayer nanoparticles and attraction between particles is strong, then the colloidal shell may have some bending rigidity such that it is able to resist buckling above a threshold pressure. If, on the contrary, the Laplace pressure is positive, even there exists interparticle repulsive force (This can also be reflected by a bigger coexisting free droplet, see Figure 3.8), the colloidal shell will deform into spherical shape (circular in 2D), despite of particle shapes. This phenomenon is reminiscent of inflation of a balloon, where the balloon is immediately spheroidized with internal pressure present even if it is small.

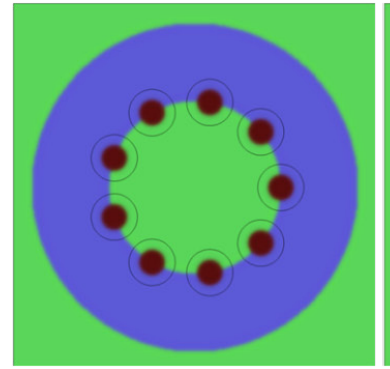

$t^{*}=0$

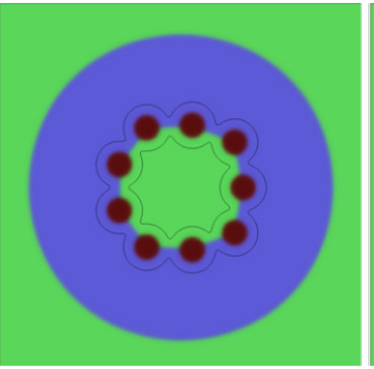

$t^{*}=3 \times 10^{5}$

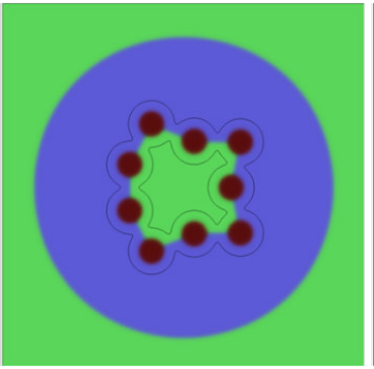

$t^{*}=6 \times 10^{5}$

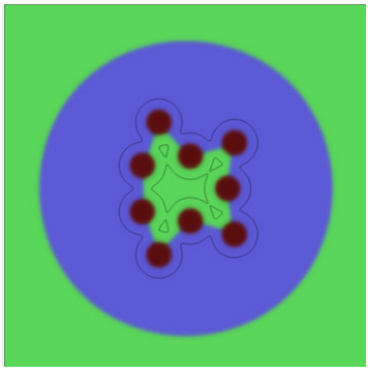

$t^{*}=1 \times 10^{6}$

Figure 3.6 Simulation results show that negative Laplace pressure leads to buckling and collapse of colloidal shell (spherical particles).

With zero Laplace pressure, however, in principle the colloidal shell may keep arbitrary morphology since the effective surface tension is zero as well, which has been verified by our simulation. In Figure 3.9, the initial morphologies of particles are predesigned enclosing an irregular droplet with a coexisting continuous phase of flat interface to maintain zero Laplace pressure. After long time diffusion processes, the particle positions/orientations are only slightly adjusted locally while the overall morphology does not change significantly, and the apparent curvature of the colloidal shell shows distinct variation in both (a) (spherical particles) and (b) (non-spherical particles). In other words, in our simulation crumpling of the colloidal shells does not mean they have finite bending rigidity. Under zero Laplace pressure, the interface tension 
acted on a particle must be balanced by interparticle forces (such as steric forces). As a result, effective surface tension is zero such that the colloidal shells can be arbitrarily and permanently distorted, such as in bijels. ${ }^{23}$ In terms of the diminished effective interfacial tension, the colloids at liquid interfaces act as surfactant. ${ }^{93}$

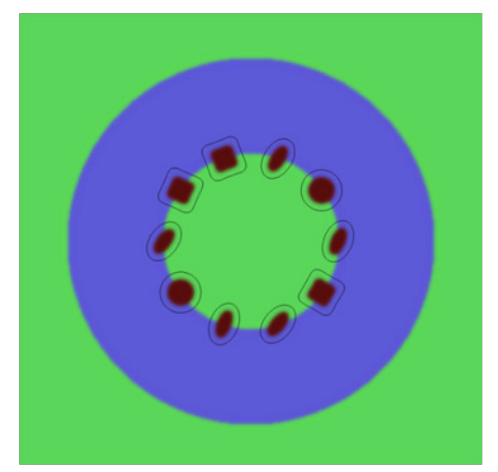

$t^{*}=0$

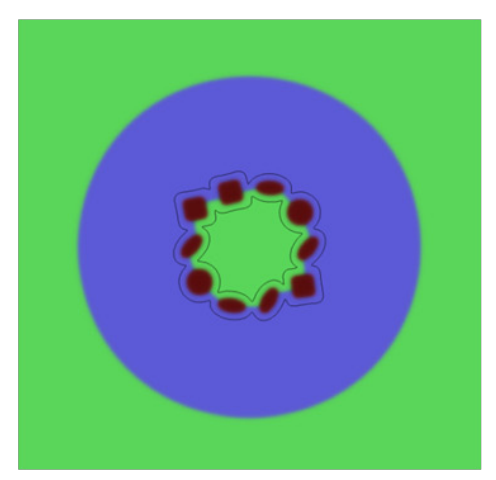

$t^{*}=5 \times 10^{5}$

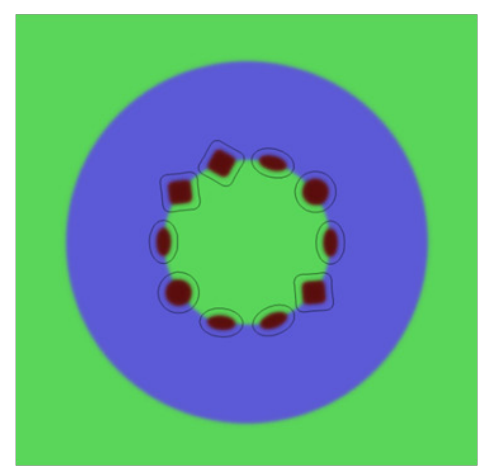

$t^{*}=2 \times 10^{4}$

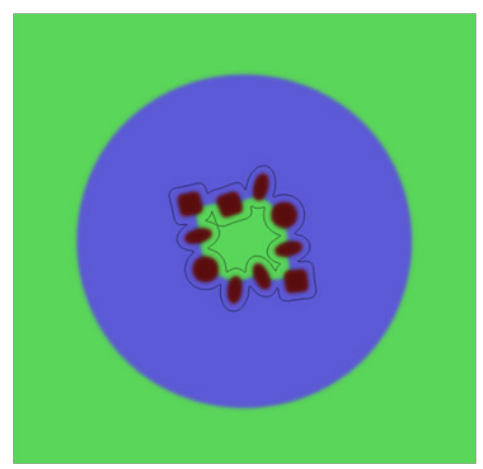

$t^{*}=1 \times 10^{6}$

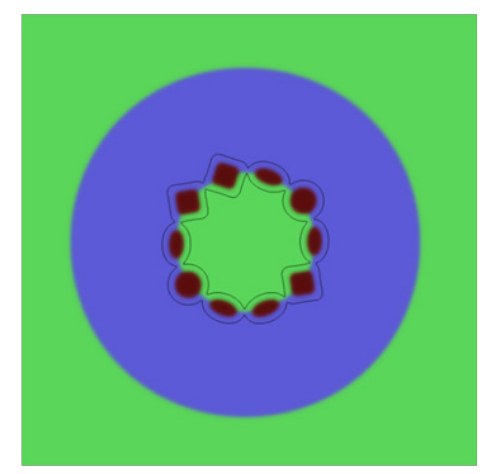

$$
t^{*}=2 \times 10^{5}
$$

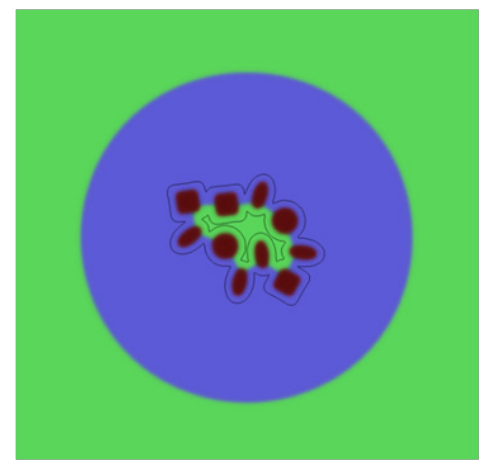

$t^{*}=2 \times 10^{6}$

Figure 3.7 Simulation results show that negative Laplace pressure leads to buckling and collapse of colloidal shell (shape-anisotropic particles of different shapes). 


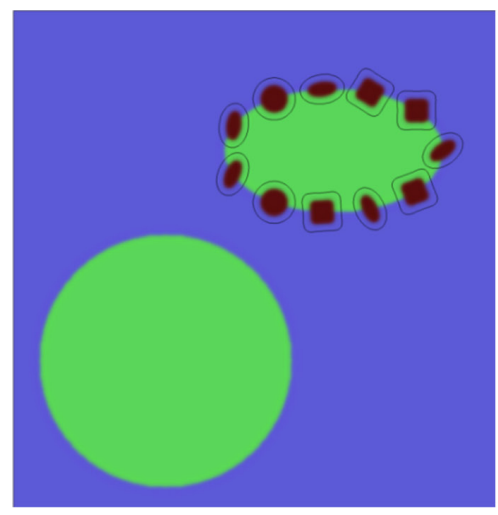

$t^{*}=0$

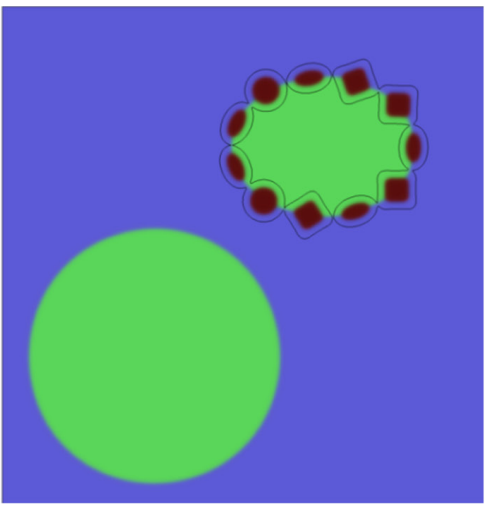

$t^{*}=1 \times 10^{5}$

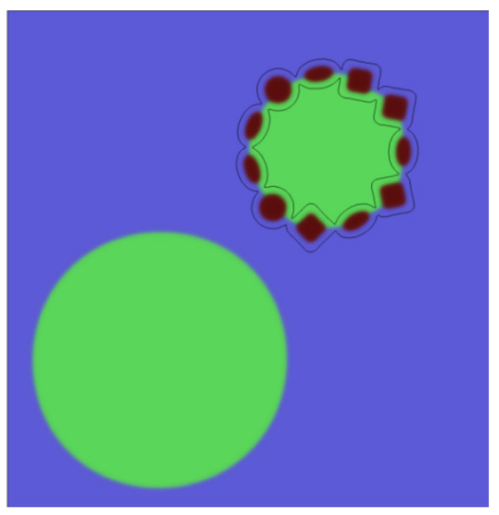

$t^{*}=1 \times 10^{6}$

Figure 3.8 Simulation results show that positive Laplace pressure leads to spheroidization of the colloidal shell. The predefined colloidal shell is nearly elliptical, and the final two drops coexist stably.
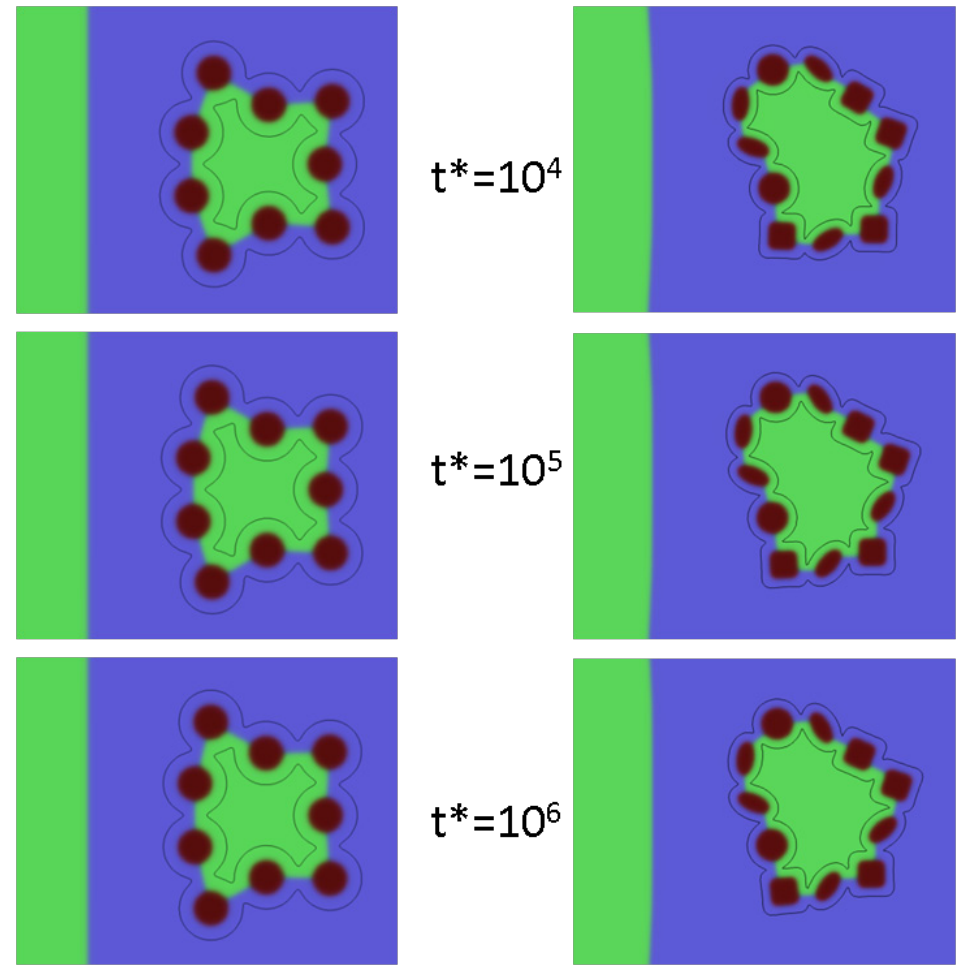

Figure 3.9 Simulation results show that colloidal shells exhibit different stable morphology with arbitrary curvature under zero Laplace pressure, which is computationally maintained by a coexisting flat interface (left green region). 


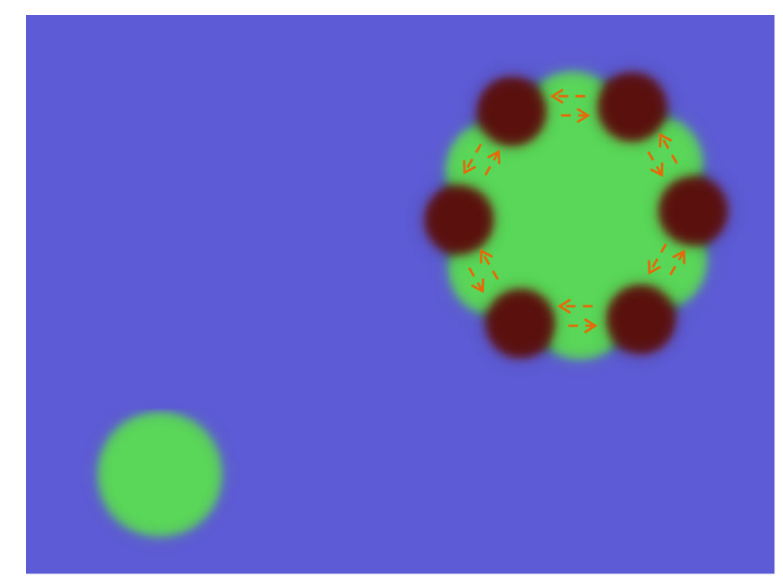

Figure 3.10 Simulation result shows that attraction between particles allows a Pickering emulsion droplet coexist with a smaller free droplet. It is seen that locally the radius of curvature of liquid interface is smaller than the apparent radius of curvature.

\section{Discussion on Laplace pressure in Bijel}

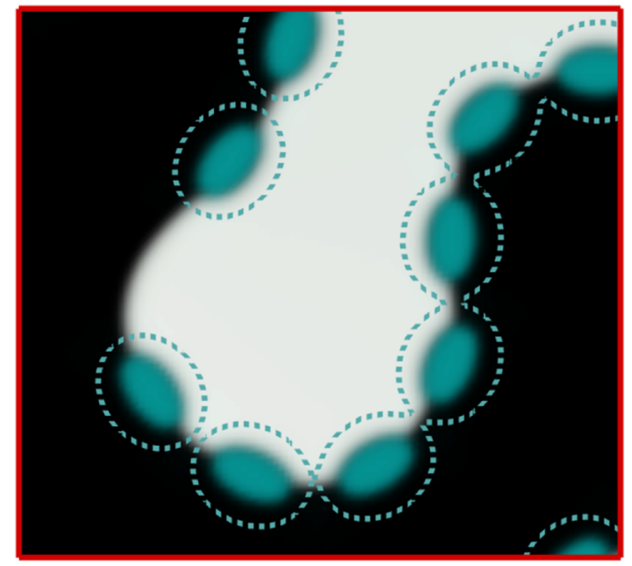

(a) $t^{*}=60,000$

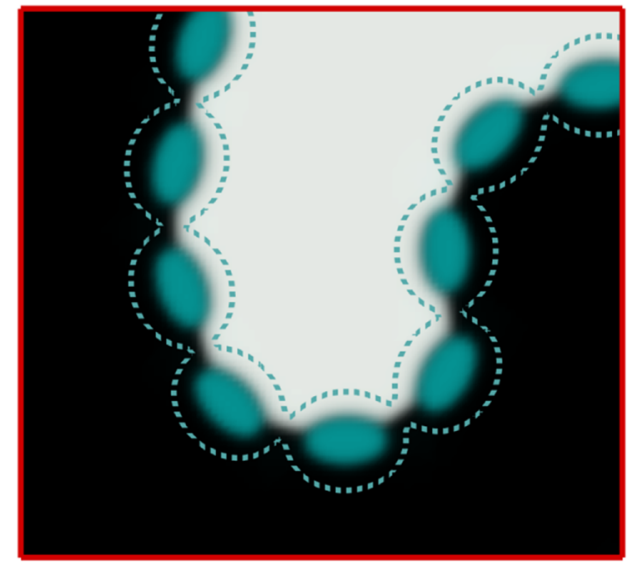

(b) $t^{*}=400,000$

Figure 3.11 Enlargement of the particle/liquid morphology in two representative zones of Figure 2.5(d) and (f), respectively. The liquid phases and particles are recolored to enhance the contrast.

The surface pressure is most significant beside naked liquid interfaces with curvature. After fully jammed, the Laplace pressure is virtually absent in the liquid. From the 
viewpoint of Young-Laplace equation, the radius of curvature is increasing to infinity, which means the liquid interfaces are almost flat. Accompanying the diminishing Laplace pressure, the interparticle repulsive forces are increasing, as is shown in Figure 3.11. In Figure 3.11a, the particles are not fully jammed, the liquid interface is partially naked and the curvature is obvious. In comparison, in Figure $3.11 \mathrm{~b}$, the particles are tightly jammed together, reflected by the partial overlapping of the "Halo" field, which indicates short range repulsive forces is significant. The liquid interfaces are almost flat. Such results indicate that the apparent radius of curvature outlined by the morphology of particles cannot be used to evaluate the Laplace pressure in the liquids. Instead the local radius of curvature should be adopted to meet the Young-Laplace equation, although it is difficult to measure experimentally. Alternatively, it can be understood by taking the effective interfacial tension as zero ${ }^{23,93}$.

The Laplace pressure is formulated in the form of Gibbs-Duhem type equation in our DIFA model. This thermodynamic pressure meets the requirement of mechanical equilibrium, characterized by the Young-Laplace equation. Simulation results show that in two dimensional cases, on isolated particles Laplace pressure exactly balances interfacial tension and maintains the mechanical equilibrium of the particles and thus keeps circular liquid interface as undisturbed, if there is no any external forces. With external forces or interparticle forces present, however, liquid interface will be deformed, which leads to attractive or repulsive lateral capillary interaction between particles. ${ }^{36}$ When colloidal particles are jamming, the actual Laplace pressure cannot be calculated by the apparent curvature of the colloidal shell (if assuming interfacial tension is unchanged from that of naked interface). The effective surface tension is decreased due to non-capillary interparticle forces. As pointed out by Clegg et al, at the jamming state interfacial tension is actually not a convenient description for the colloidal shell, since the free energy change per surface area is very sensitive to the short-range non-capillary interparticle forces. ${ }^{23}$ However, it is shown in our simulation that the actual Laplace pressure is indeed detectable by measuring the curvature of a stable coexisting free droplet enclosing the same liquid phase. For monolayer particles coated Pickering 
emulsion, positive Laplace pressure leads to a spheroidized colloidal shell at equilibrium; negative Laplace pressure leads to collapse and shrinkage of the colloidal shell; and distorted colloidal shells with irregular morphology may stay at zero Laplace pressure due to zero effective surface tension. In all previous simulations interparticle force is assumed to be repulsive. It needs to be emphasized if interparticle force is attractive (see Figure 3.10, where a common Lennard-Jones potential function between particles is defined and the interparticle distance is larger than the equilibrium one), a monolayer Pickering emulsion droplet can coexist with a smaller free droplet. The microscopic local curvature of liquid interface is larger than the apparent curvature of the particle contour, which means the effective surface tension of Pickering emulsion is larger than of naked liquid interface. Without attraction between particles, by Ostwald ripening the Pickering emulsion droplet will expand at the expense of the small droplet and the latter will finally disappear.

\subsection{Stability of capillary bridges through Communicating Vessels Effect $^{37}$ *}

\subsubsection{Introduction}

A small amount of liquid bridging the wettable or partially wettable surfaces of two solid particles may produce strong adhesion between the particles due to surface tension as well as Laplace pressure. ${ }^{38,78,94}$ Such liquid bridges of pendular shape, so-called capillary bridges, play important roles in various places, such as plastic-like wet sand/soil ${ }^{95,}{ }^{96}$ and adhesive toe pads of some small animals. ${ }^{97}$ At small length scales capillary forces typically dominate over particle gravity. ${ }^{29,78,98,99}$ Based on capillarity effect, an electronically controlled switchable adhesion device was recently engineered. ${ }^{100}$ Most studies focused on capillary bridges formed by liquid in vapor

* The main content of this section has been published on Langmuir (2012), 2696-2703. 
through condensation, while capillary bridges can also be formed by two immiscible liquid phases. If the two liquid phases are insoluble in each other, mechanical agitation needs to be employed to distribute the preferential liquid phase into the tiny gaps between particles to form liquid bridges. Such a method has been used to demonstrate that mixing a small amount of a second insoluble liquid into a colloid suspension can dramatically change its rheology from fluid-like to gel-like. ${ }^{98}$ However, mechanical agitation inevitably changes particle arrangements thus cannot be used to introduce capillary bridges into well-formed particle microstructures. An appealing way to introduce capillary bridges is through binary liquid phase separation controlled by composition and temperature. ${ }^{101}$ Such a method is expected to be able to stabilize engineered particle microstructures. Nevertheless, a serious concern is the stability of such formed capillary bridges due to liquid clustering phenomena frequently observed in wet particulate materials, ${ }^{101-103}$ since coarsening of the dispersed phase is a general phenomenon during phase separation, also known as Ostwald ripening. ${ }^{104,105}$ Existing relevant theoretical work and molecular level simulations mostly focus on the kinetic process of an isolated capillary bridge in given ambient vapor pressure, ${ }^{106-109}$ or the macroscopic bulk phase transition shift due to capillary effect. ${ }^{110,111}$ In the well-studied capillary condensation problem, the equilibrium state of a liquid meniscus is described by the Kelvin equation valid down to a few nanometers of meniscus radius, ${ }^{112,113}$ which links individual radii of curvature of liquid surface to the ambient vapor pressure. Because the ambient vapor pressure is usually constant, the interaction among different menisci is rarely considered. In a two-liquid-phase colloid system, however, a meniscus is strongly interacting with adjacent menisci through inter-liquid diffusion. Such interactions among adjacent capillary bridges and the effects on their stabilities during kinetic growth processes are still inadequately studied.

In this section, we focus on multiple mutually interacting capillary bridges spontaneously formed between closely packed colloidal particles in phase separating liquids. Computer simulations are performed based on a DIFA. ${ }^{65}$ A self-stabilization mechanism is revealed that operates through diffusive equilibrium of two-phase liquid 
morphologies, where adjacent capillary bridges interact with each other through diffusion in analogy to the liquid interaction in communicating vessels through flow. Such a selfstabilization mechanism automatically stabilizes the spontaneously formed capillary bridges between neighboring colloidal particles during liquid solution phase separation, in contrast to conventional coarsening processes. ${ }^{114}$ It renders the capillary bridges desired microstructural stability and uniformity, which are important for a practical route to in-situ (in-liquid) firming of fragile colloidal crystals and other compact colloidal microstructures by using capillary bridges.

\subsubsection{Computational details}

The semi-implicit spectral method (Section 2.3) is employed to solve the CahnHilliard equation, which offers significantly improved efficiency and accuracy comparing to the explicit Euler scheme. The time increment is set to $\Delta t=0.5 l^{2} /(\Delta H M)$, where $l$ is computational grid size, $\Delta H$ is enthalpy of mixing of the binary solution, and $M_{\alpha}=M$. In the simulations, solid particles in the compact colloidal microstructures are immobile. Thus, the $\left\{\eta_{\beta}\right\}$ field variables are fixed in space and do not evolve. It is worth noting that a unique capability of DIFA model is to treat motions of arbitrary-shaped colloid particles under influences of various forces, including capillary, steric and electrostatic

forces, as previously demonstrated; ${ }^{22,24}$ however, the focus of this work is on the spontaneous formation of capillary bridges in compact colloidal microstructures where solid particles are pre-assembled and immobile.

Accumulated round-off errors during numerical solution of diffusion equation over long time (large number of iterations) may generate non-negligible composition drift. The concentration-correction scheme is adopted to guarantee mass conservation (Section 2.3). While such a correction is negligibly small for each time increment, it is necessary for simulations over a large number of iteration steps, as in our case where capillary bridge 
formation during phase separation is very sensitive to the volume fraction of the minor phase.

In simulations the parameters $A$ and $\chi$ are set to $1 / 12$ and 1 , respectively, and $\lambda_{A}=0.5, \lambda_{B}=2.4, \kappa_{A}=0.4, \kappa_{B}=1.0$, which give approximately $3 l$ thickness of all diffuse interfaces between $10 \%$ and $90 \%$ of full equilibrium value, and interface energy (normalized by $\Delta H l$ ) $\gamma_{A B}=0.262$ for liquid phase A-liquid phase B, $\gamma_{A S}=0.108$ for liquid phase A-solid particle, and $\gamma_{B S}=0.240$ for liquid phase B-solid particle. According to Young's equation, the contact angle of liquid interface at solid surface is $\theta=\cos ^{-1}\left[\left(\gamma_{B S}-\gamma_{A S}\right) / \gamma_{A B}\right] \approx 60^{\circ}$. This contact angle is used in this work. Periodic boundary condition is employed for all simulations. While the DIFA model is formulated for three dimensions, computer simulations here are performed in two dimensions due to the large amount of computing time required to solve diffusion equation over entire phase separation processes. It is worth noting that, in two-dimensional simulations, the circular particles are not spheres but rather cylinders of infinite length, thus a capillary bridge between two cylinders has only one curvature, while a capillary bridge between two spherical particles has two curvatures. For small capillary bridges formed between closely packed particles considered in this paper, the azimuthal radius is much greater than the meridional radius, thus only one curvature is significant in determining the negative Laplace pressure in the capillary bridge.

\subsubsection{Simulation results and discussions}

\subsubsection{Simulation of capillary bridge formation in colloidal crystal}

We first study the formation of capillary bridges in an ordered colloidal crystal. The simulation starts from a homogeneous binary liquid solution that undergoes a fast cooling 
into the miscibility gap, with thermally induced small fluctuations to initiate the phase separation process.

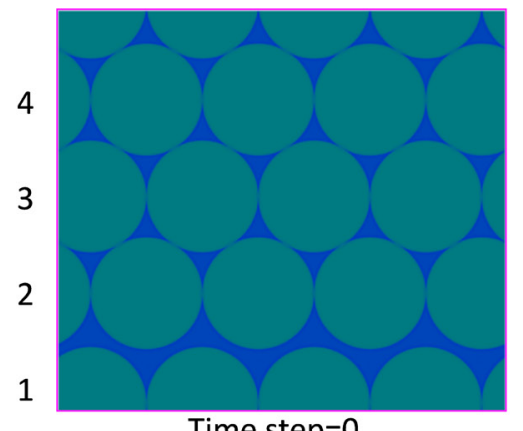

Time step $=0$

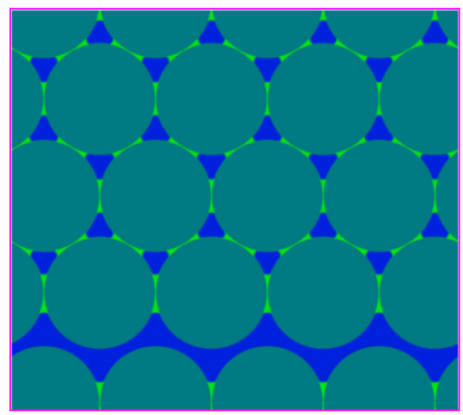

Time step $=150,000$

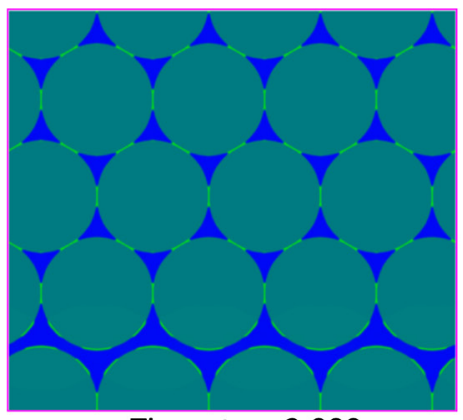

Time step $=2,000$

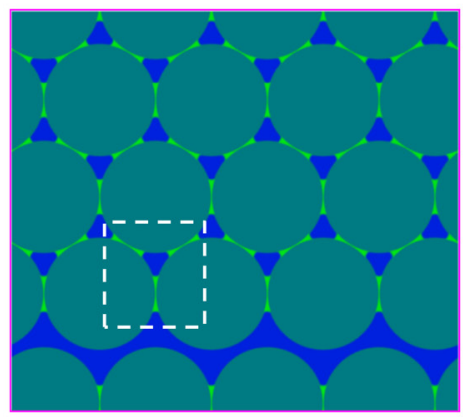

Time step $=500,000$

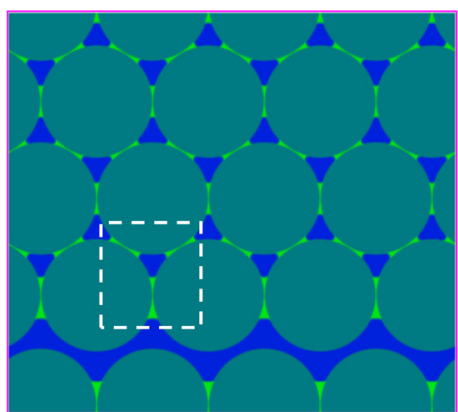

Time step $=50,000$

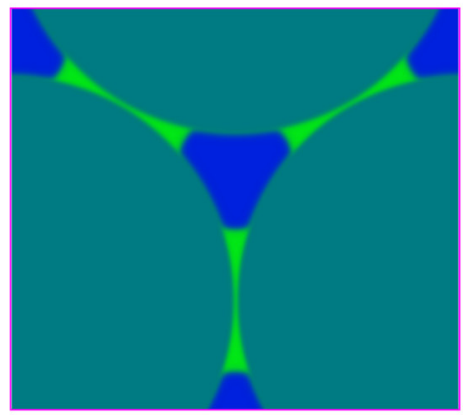

Detailed view of the last stage

Figure 3.12 Simulated nucleation and growth of capillary bridges formed in colloidal crystal during liquid phase separation process. Colloidal particle volume fraction $f_{p}=0.86$. Total fraction of phase A in liquid (fully separated) is $\Phi_{A}=1-\Phi_{B}=0.18$. Two-phase liquid morphology reaches equilibrium after 500,000 time steps. Last panel shows the detailed stable capillary bridges at 500,000 time steps. Colors stand for different liquid phases (green, blue) and particles. (See Movie S1 in Supporting Information for full evolution process.)

It has been known that when the supersaturation reaches a threshold, ${ }^{115,116}$ surface adsorption of the preferential liquid species is strong enough, ${ }^{116,117}$ or the inter-particle distance $D$ is sufficiently small (comparable to the thickness of the wetting film), ${ }^{108,110}$ nucleation of the capillary bridges is virtually barrier-free. In our simulation, the preferential adsorption at particle surfaces leads to heterogeneous nucleation in the 
narrow gaps between solid particles throughout the system, forming small capillary bridges. Subsequent decomposition of the supersaturated solution leads to growth of these capillary bridges. Most importantly, as shown in Figure 3.12, the simulation reveals a local stabilization mechanism of the capillary bridges that operates through diffusive equilibrium of two-phase liquid morphologies. Such mechanism renders desired microstructural stability and uniformity to the capillary bridges that are spontaneously formed in colloidal crystals during liquid solution phase separation.

Figure 3.12 shows the simulated time evolution of liquid phase separation process and capillary bridge formation in a colloidal crystal. Space is discretized into $912 \times 816$ computational grids, with particle diameter of $226 l_{0}$ to adquately resolve the gap spaces between particles. All particles are in almost contact (numerically there is a small distance of about the diffuse interface thickness betweeb adjacent solid surfaces). An ordered colloidal crystal microstructure is assumed, except for rows 1 and 2 with a broader spacing to intentionally introduce certain degree of structural nonuniformity. It is observed that capillary bridges of liquid phase A (with greater wettability to particle surface) nucleate in the narrowest slits between particles as assisted by the surface adsorption, and the menisci grow gradually. Due to the broader gaps between particles in rows 1 and 2, while surface adsorption occurs on particles (time step 2,000), capillary bridges are not formed there; instead, the abundant liquid component $\mathrm{A}$ in this region diffuses away to feed the growth of adjacent capillary bridges that become bigger than furtheraway ones during growth (time step 50,000). In particular, the capillary bridges in row 2 are initially not only bigger but also unsymmetric as compared with those in rows 3 and 4, as highlighted at time step 50,000. However, with phase separation proceeded, these bigger and unsymmetric capillary bridges not only become symmetric but also shrink to the same size of other ones (time step 150,000). In contrast to conventional coarsening processes during phase separation, the capillary bridges do not coarsen or coalesce; instead, they mutually interact through diffusion to balance each other to achieve self-stabilization. When equilibrium is reached in the simulation after time step 500,000 , all capillary bridges grow into a uniform size, despite the imperfection (broader 
spacing between rows 1 and 2) present in the colloidal crystal. The final stable morphology of liquid phase A and solid particles resembles a mortar-brick structure in architecture. In the following sections, we analyze the self-stabilization mechanism of capillary bridges and calculate the adhesion strength of capillary bridge-reinforced colloidal crystal.

\subsubsection{Analysis of self-stabilization mechanism of capillary bridges}

(a)

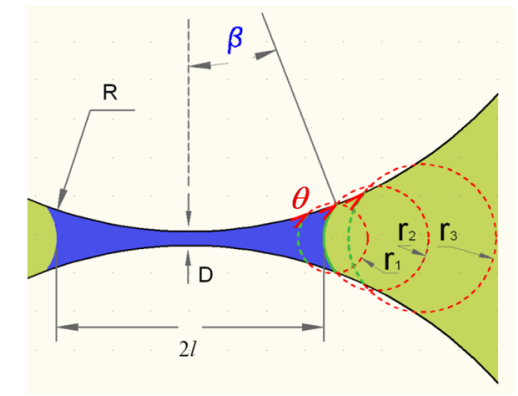

(b)

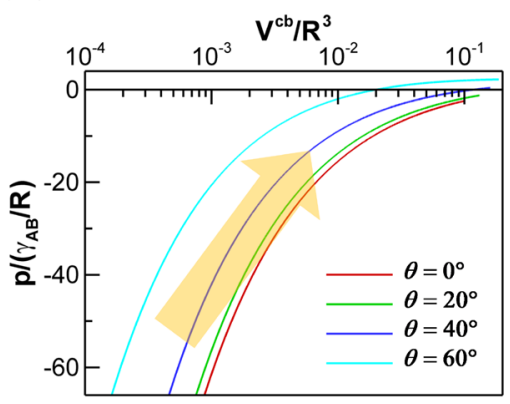

(c)

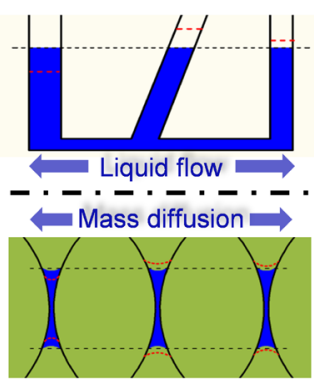

Figure 3.13 (a) Schematic of a capillary bridge between two solid spheres. Blue and green regions are coexisting liquid phases $\mathrm{A}$ and $\mathrm{B}$, respectively, with contact angle $\theta<90^{\circ}$ (measured in phase A). As small meniscus between spheres grows outward, its radius of curvature $r$ increases $\left(r_{1}<r_{2}<r_{3}\right)$ due to geometrical constraint. (b) The Laplace pressure (negative) inside a capillary bridge is a monotonically increasing function of capillary bridge volume $V^{c b}$ (for acute contact angle much smaller than $90^{\circ}$ ). Here $D$ is set to zero, for nonzero small $D$ the trend is the same, see Figure 3.22 in Section 3.6. (c) Analogy between Laplace pressure stabilizing meniscus size through inter-liquid diffusion (bottom) and hydrostatic pressure equilibrating liquid level through flow in communicating vessels (top).

Consider two spherical particles of radius $R$ with a small separation $D(D \ll R$, Figure 3.13a) immersed in a two-phase liquid. Assume the particles are more lyophilic to liquid phase $\mathrm{A}$, and phase A forms a pendular meniscus within the narrow slit. In 2D case, 
the Laplace pressure across the liquid interface of capillary bridge is simply $\Delta p=-\gamma_{A B} / r$, where $\gamma_{A B}$ is the interfacial tension between liquid phases $\mathrm{A}$ and $\mathrm{B}$, and $r$ is the radius of curvature of the meniscus, which is dictated by the contact angle $\theta$ and the two-sphere surface geometry. The geometric confinement of the narrow slit between the two spheres prescribes that $r$ increases as the capillary bridge grows bigger in order to maintain the correct contact angle (Figure 3.13a). This means that the pressure inside the pendular meniscus monotonically increases as the capillary bridge grows bigger. To be general, we analyze 3D case (see supplementary material in 3.6.1 for 2D analysis that exhibits qualitatively similar behavior). In $3 \mathrm{D}$, by using the commonly adopted circular approximation, ${ }^{38,78}$ i.e., assuming the meniscus profile along the axis of symmetry is described by a circular arc, the Laplace pressure inside the meniscus (internal pressure of liquid phase A with respect to external bulk pressure of liquid phase B) is given by Young-Laplace equation

$$
p=\gamma_{A B}\left(\frac{1}{l}-\frac{1}{r}\right)
$$

where $l$ and $r$ are respectively the azimuthal and meridional radii of the meniscus. Since usually $r \ll l$, the Laplace pressure is dominated by the meridional radius $r$, just like in $2 \mathrm{D}$ case (It is worth noting that circular approximation is no longer an approximation but is a requirement for equilibrium in two dimensions). Figure $3.13 \mathrm{~b}$ plots the quantitative dependence of Laplace pressure on capillary bridge volume at different contact angles (see supplementary material in 3.6.1 for calculation details), which is practically the same as the exact solutions obtained without circular approximation by Heady and Cahn. ${ }^{38}$ This plot emphasizes that capillary bridges defy the conventional coarsening process as usually observed during phase separation, in which Laplace pressure inside a droplet decreases as it grows bigger, thus bigger droplets grow at the expense of smaller ones. In capillary bridges, however, inter-liquid diffusion shrinks bigger bridges (with higher internal pressure) and feeds the growth of smaller ones (with lower internal pressure), which naturally creates a negative feedback for the growth of capillary bridges and automatically equilibrates their sizes. Such a self-stabilization mechanism of pressurevolume relationship with positive slope in capillary bridges (Figure $3.13 b$ ) resembles the 
role of Pascal's law ( $\Delta p=\rho g \Delta h)$ in communicating vessels to equilibrate liquid levels (Figure 3.13c).

It is worth noting that the above-discussed stability associated with the negative Laplace pressure inside the capillary bridges does the well-known Ostwald ripening work but in the opposite direction when the particles have negative curvature. In the context of classical works on Ostwald ripening, ${ }^{104,105}$ dispersed particles possess positive mean interfacial curvature as averaged over the entire surface, where Laplace pressure decreases with increasing particle volume, leading to coarsening. In the case of capillary bridges, instead of dispersed droplets, liquid bridges are formed in small gaps between solid particle surfaces, and it is such geometrical constraints that render capillary bridges negative mean interfacial curvature as averaged only over the liquid-liquid surface but not the liquid-solid surface, where Laplace pressure increases with increasing bridge volume, leading to the aforementioned stability. To apply the analysis of Ostwald ripening to capillary bridges, the effects of such geometrical constraints must be taken into account, which are absent in the cases of dispersed particles/droplets.

The Laplace pressure across a curved interface leads to chemical potential shift in the liquid mixture in accord with the Gibbs-Duhem relation. ${ }^{76}$ For the capillary bridges of liquid phase $\mathrm{A}\left(c_{1} \approx 1\right)^{118}$ in our simulation, the chemical potential $\mu_{1}=\delta F / \delta c_{1}$ reflects the internal pressure. ${ }^{67}$ Figure 3.14 plots the chemical potential distribution inside the capillary bridges in the highlighted box shown in Figure 1 at time step 50,000. It clearly shows that $\mu_{1}$ is higher and nonuniform inside the bigger and unsymmetric capillary bridge as compared to that in the other two capillary bridges. It is this nonuniform chemical potential distribution that drives the bigger bridges to shrink and become symmetric. As a result, all capillary bridges interact with each other through inter-liquid diffusion and ultimately achieve equilibrium of uniform pressure and chemical potential. Such an effect is of critical importance since it implies an intrinsic self-stabilization mechanism among adjacent capillary bridges through diffusion. Therefore, the simulated 
formation of self-stabilized capillary bridges shown in Figure 3.12 is not by chance but a spontaneous process. In some systems, the phase separation process can be reversed through convenient temperature control. ${ }^{101,} 119$ Many binary liquid systems, such as alchol-oil ${ }^{23}$ and water-lutidine, ${ }^{22}$ have a critical phase separation temperature close to room temperature, making it possible for the capillary bridge-reinforced colloidal crystal to be used at room temperature. The simulation thus indicates a potential route to in-situ firming of colloidal crystals, whose adhesion strength is evaluated in the next section.

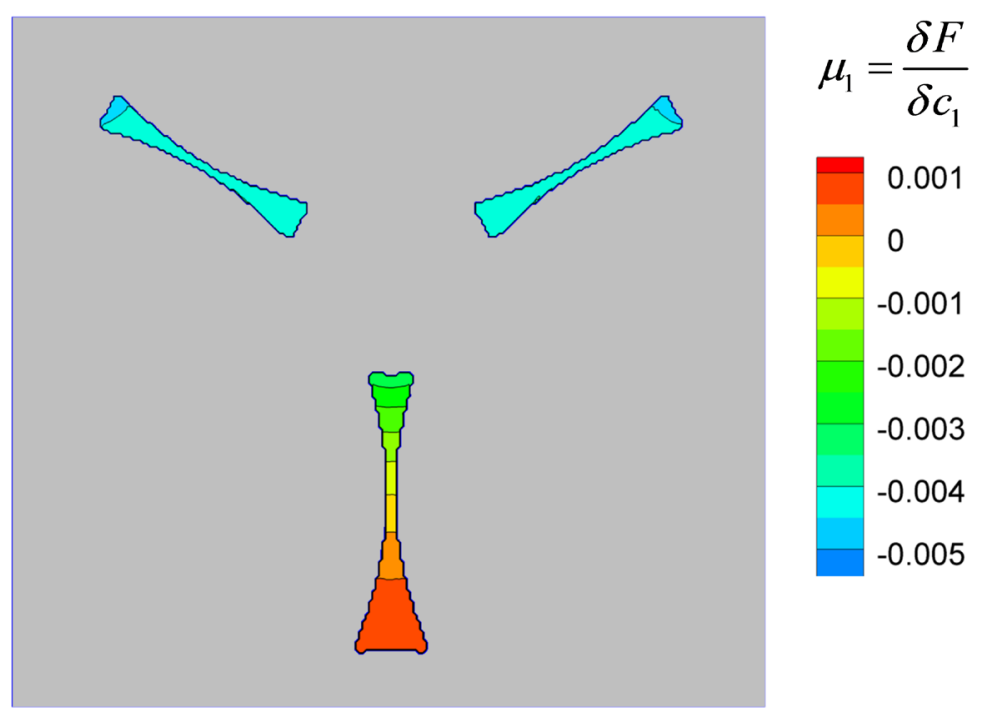

Figure 3.14 Contour plot of chemical potential $\mu_{1}=\delta F / \delta c_{1}$ distributed inside the capillary bridges in the highlighted box shown in Figure 3.12 at time step 50,000, reflecting internal pressure of the capillary bridges.

\subsubsection{Adhesion strength of capillary bridge-reinforced colloidal crystal}

The total attractive capillary force between two spheres in contact is $F^{a d h}=\omega \gamma_{A B} R,{ }^{38}$ where $\omega$ is a coefficient; for capillary bridge volume $V^{c b} \sim 0.01 R^{3}$ or less, $\omega$ is approximately 5 for contact angle of $0^{\circ}$ and 2 for contact angle $60^{\circ}$. Consider a closely packed 3D colloidal crystal of face-centered cubic (FCC) symmetry, where all 
neighbouring spherical particles are bonded by capillary bridges. Consider a sphere on the (100) face center of FCC unit cell, which experiences attractive forces in the ${ }^{120}$ direction from 4 adjacent external spheres outside the unit cell exerted by capillary bridges with a total magnitude of $4 \times \sqrt{2} F^{\text {adh }} / 2=2 \sqrt{2} F^{\text {adh }}$. There are two spheres on the (100) face of area $2 \sqrt{2} R \times 2 \sqrt{2} R=8 R^{2}$, thus the force density on (100) plane is $2 \times 2 \sqrt{2} F^{a d h} / 8 R^{2}=\sqrt{2} F^{a d h} / 2 R^{2}$. Given the cubic symmetry, the average compressive stress contributed solely from capillary bridges is

$$
\sigma_{i j}=-\sqrt{2} F^{a d h} \delta_{i j} / 2 R^{2}
$$

where $\delta_{i j}$ is the Kronecker delta. Substituting $F^{a d h}=\omega \gamma_{A B} R$ into Eq. (3.5) yields the tensile strength of the colloidal crystal as reinforced by the capillary bridges

$$
\sigma^{T S}=\frac{\sqrt{2} \omega}{2} \frac{\gamma_{A B}}{R}
$$

The above formula indicates an inverse scaling of the tensile strength of the colloidal crystal with respect to particle radius, which is bounded by a theoretical limit of negative pressure that the liquid can sustain before cavitation (typically when the particle size reduces to nanometers)..$^{99}$ Macrosopically, the adhesion caused by the self-stabilized uniform capillary bridges exhibits high degrees of isotropy and homogeneity. Assuming $\gamma_{A B}=10^{-3} \mathrm{~J} / \mathrm{m}^{2}$ and $R=100 \mathrm{~nm}$, then the tensile strength can reach the order of magnitude of $10^{4} \mathrm{~Pa}$, rendering sufficient strength to the colloidal crystals for many practical applications, such as 3D photonic crystals. Moreover, the strong capillary bridges would pull particles into more intimate contact and cause strengthening of van der Waals forces between the particles (see recent findings on the frozen colloid network after jamming at fluid interfaces ${ }^{121}, 122$ ). With appropriate particle surface properties, sintering ${ }^{123}$ between particles can be controlled to further reinforce the structures if needed. A unique merit of the pendular capillary bridge reinforcement, in addition to its large strength, is that ideally the strong adhesion only increases equal normal contact stress between particles without introducing unwanted shear stress or torque to distort the 
original structure (as reported in experiment ${ }^{101}$, the randomly packed microspheres did not undergo perceivable movement until coalescence of capillary bridges). Thus, the symmetry of the capillary bridge-reinforced colloidal crystal can be well preserved.

\subsubsection{Volume fraction limit of capillary bridge liquid phase}

The plot of internal pressure as a function of capillary bridge volume in Figure 3.13b is obtained based on the assumption of isolated capillary bridge, which breaks down if neighboring menisci meet together. For spherical particles closely packed in facecentered cubic crystal, coalescence of neighboring menisci occurs when the filling angle $\beta>30^{\circ}$ (see Figure 3.23 in supplementary material in 3.6.1 for critical capillary bridge volume). By calculating the critical capillary bridge volume $V^{c b *}$, the critical volume fraction of liquid phase $\mathrm{A}\left(\Phi_{A}{ }^{*}\right)$ in the liquid mixtures is given by

$$
\Phi_{A}{ }^{*}=\frac{N_{c} f_{p} V^{c b^{*}}}{2\left(1-f_{p}\right) V_{p}}
$$

where $V_{p}$ is the volume of a particle, $f_{p}$ is particle volume fraction, and $N_{c}$ is the coordination number of the crystal (each particle possess $N_{c} / 2$ capillary bridges). It is worth noting that, as a capillary effect, the Laplace pressure causes a slight composition shift from the equilibrium composition of bulk phase diagram, ${ }^{110,111}$ which needs to be taken into account when using level rule to precisely control the volume of minor phase based on the binary phase diagram. Moreover, if $\Phi_{A}$ is below but very close to $\Phi_{A}{ }^{*}$, there could still be a chance for capillary bridges to coalesce locally, especially in randomly packed colloidal structures due to local oversupplies of minor phase species and limited diffusion range, as demonstrated in Figure 3.15a. Once coalescence occurs, the liquid phase B is locally trapped and isolated from the particle surface, resulting in loss of the contact angle and interfacial tension. The droplets of phase B eventually shrink to disappear due to regular coarsening, leading to liquid clusters of phase A. Nevertheless, the internal pressure of the liquid clusters is determined by the curvature of the liquid interface of the clusters, thus equilibrium is reached between clusters and 
capillary bridges preventing overgrowth of clusters. However, such stabilized clusters are local defects since they locally weaken the adhesion strength of the capillary bridgereinforced colloidal structures. In order to avoid local clustering and form uniform capillary bridges to reinforce imperfect colloidal structures, $\Phi_{A}$ should be chosen sufficiently below $\Phi_{A}{ }^{*}$, as demonstrated in Figure 3.15b.

(a)

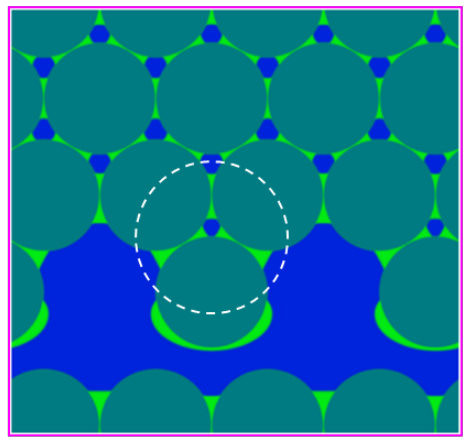

Time step $=200,000$

(b)

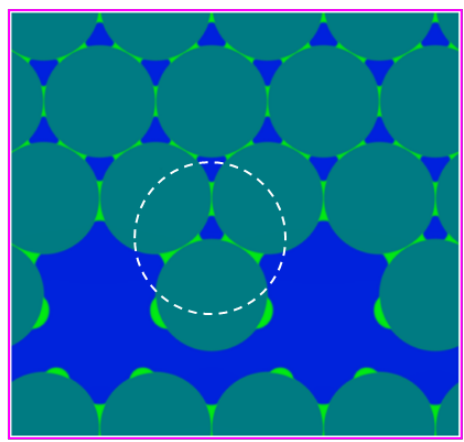

Time step $=50,000$

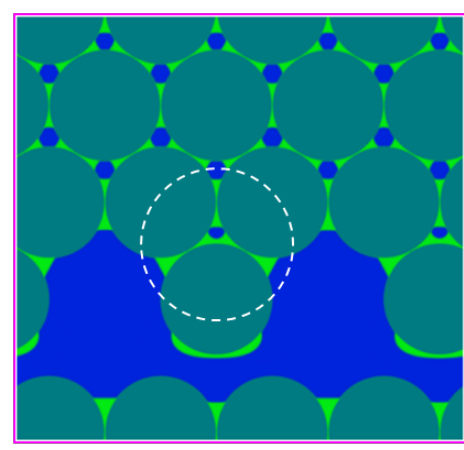

Time step $=400,000$

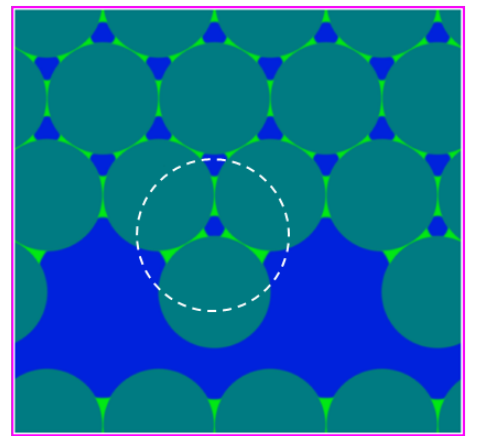

Time step $=150,000$

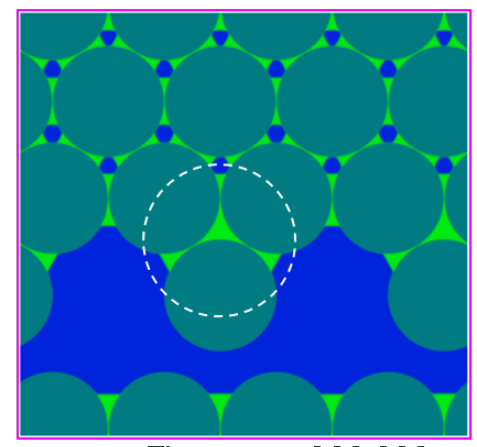

Time step $=800,000$

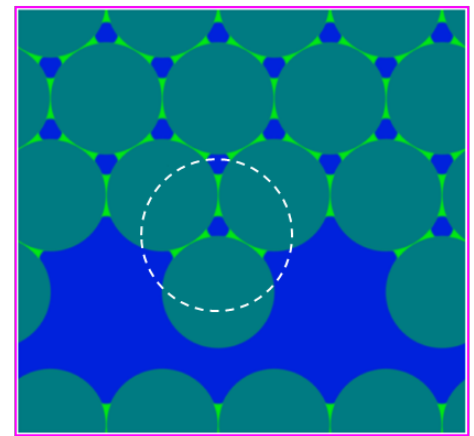

Time step $=600,000$

Figure 3.15 Simulated capillary bridge formation during liquid phase separation in imperfect colloidal crystal, where two particles are removed in addition to increased distance between rows 1 and 2 (colloid volume fraction $f_{p}$ thus reduces to $71 \%$ ). $\Phi_{A}{ }^{*}$ in this $2 \mathrm{D}$ configuration is about 0.20 (Note that $\Phi_{A}{ }^{*}$ corresponding to configuration in Figure 3.12 is about 0.50 ). (a) $\Phi_{A}=0.18$ close to $\Phi_{A}{ }^{*}$, and capillary bridges inside the highlighted region coalesce and form clustered liquid phase A. (b) $\Phi_{A}=0.10$ well below $\Phi_{A}{ }^{*}$, thus coalescence of capillary bridges and liquid clustering are avoided, leading to uniform-sized capillary bridges. 


\subsubsection{Thermodynamic stability of capillary bridges}

As shown in Figure 3.15a, a liquid cluster can also self-stabilize with respect to other capillary bridges as well as other liquid clusters. An interesting question is whether the uniform pendular state (as shown in Figure 3.12) is the lowest free energy state as compared to clustered states. In order to answer this question, we perform an imaginary relocation operation in which one block of liquid phase B between capillary bridges (see the triangular blue region in the last panel of Figure 3.12) is replaced with liquid phase A by equally extracting liquid A from all $N$ capillary bridges, and allow the system to relax and reach new equilibrium. Then the total system energies of the two states are compared, i.e., uniform capillary bridge state and nonuniform state with one liquid cluster. Similar operations are also performed for nonuniform states of two and three liquid clusters. Figure 3.16 shows these different states and compares their total system energies relative to the uniform state. The uniform capillary bridge state has the lowest energy, thus is thermodynamically stable. This conclusion holds when $\Phi_{A}$ is much smaller than $\Phi_{A}{ }^{*}$.

The same conclusion of thermodynamic stability for uniform capillary bridge state can also be reached analytically. In above imaginary relocation operation, the replacement of the liquid phase $\mathrm{B}$ (of volume $\Delta V$ ) by liquid phase A reduces interfacial energy in that region by $-\Delta E^{\mathrm{int}}$, whereas there is interfacial energy increase $\delta E^{c b}$ associated with each capillary bridge due to its volume change $\delta V^{c b}=-\Delta V / N$, where $N$ is the number of capillary bridges, while the bulk energy change is zero to the first-order approximation due to diffusive equilibrium in the original state. The excess free energy of the whole system during this imaginary process is thus mainly from interfacial energy changes

$$
\Delta F=-\Delta E^{\mathrm{int}}+N \cdot \delta E^{c b}
$$




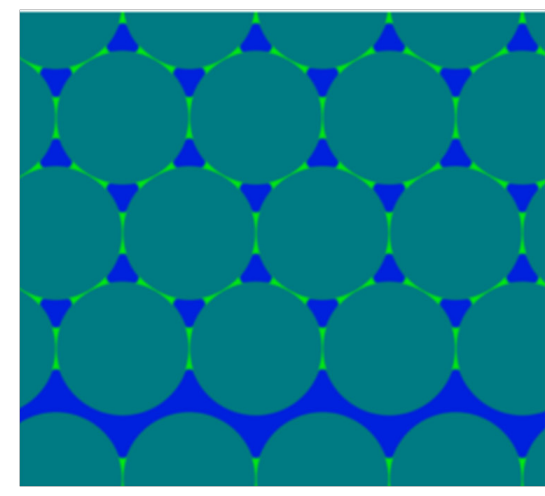

(a) $\Delta F^{*}=0$

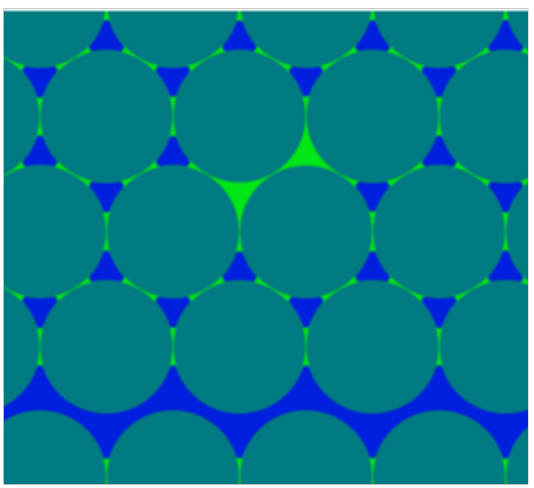

(c) $\Delta F^{*}=27$

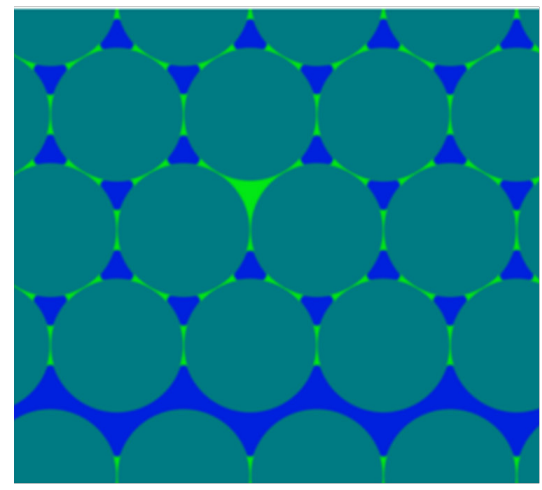

(b) $\Delta F^{*}=11$

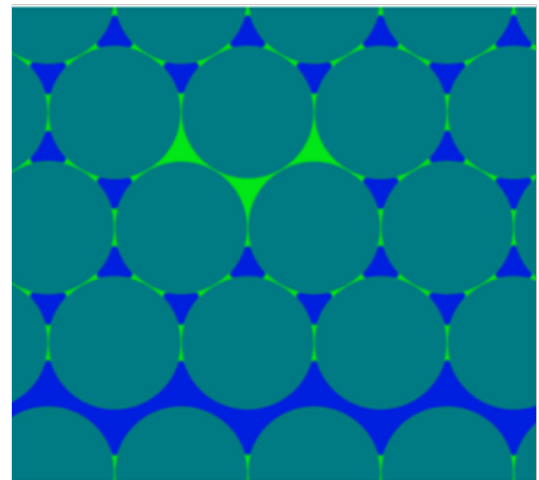

(d) $\Delta F^{*}=49$

Figure 3.16 Comparison of simulated free energies of different liquid phase distributions in stable and metastable states. The same volume fraction $\Phi_{A}=0.14$ of liquid phase A and particle configuration are used in all cases. The uniform capillary bridge state (first panel, without clustering) has the lowest energy.

where

$$
N \cdot \delta E^{c b}=N \cdot \frac{d E^{c b}}{d V^{c b}} \delta V^{c b}=-\frac{d E^{c b}}{d V^{c b}} \Delta V
$$

The first term in Eq. (3.8) is negative, while the sign of the second term depends on $d E^{c b} / d V^{c b}$. The dependence of capillary bridge interfacial energy $E^{c b}$ on the bridge volume $V^{c b}$ is plotted in Figure 3.17, which shows that $d E^{c b} / d V^{c b}$ is mostly negative (except for large contact angle) with absolute value rapidly increased for small $V^{c b}$. This implies that the second term in Eq. (3.8) is positive and dominates over the first term in 
$\Delta F$ for small $V^{c b}$. Thus, when capillary bridge volume is small, $\Delta F>0$, and the uniform capillary bridge state has the lowest energy than nonuniform states with clustering of liquid phase A. This conclusion is in agreement with the simulation results shown in Figure 3.17.

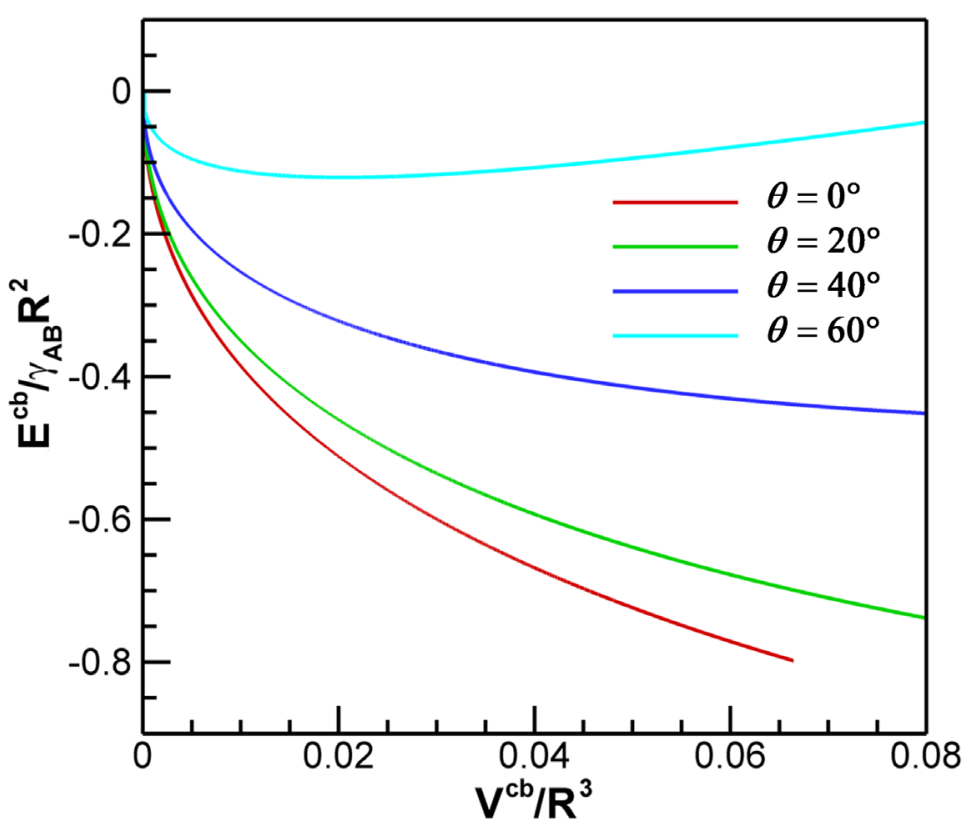

Figure 3.17 Dependence of interfacial energy associated with a capillary bridge on the capillary bridge volume. (see supplementary material in 3.6.1 for calculation details)

It is worth mentioning the Rayleigh-Plateau instability in relation to the capillary bridges. The well-known Rayleigh-Plateau instability states that a slim cylindrical column of liquid will lose stability and spontaneously breakup. ${ }^{124,}{ }^{125}$ In the cases of capillary bridges between moving particles, if the inter-particle distances could approach the Rayleigh-Plateau stability limit, seriously stretching the liquid bridges, then threedimensional simulations are required to take into account the second curvature which may cause pinch-off of the liquid column. However, for capillary bridges between closely packed fixed particles considered in this paper, the geometry of capillary bridges is well below the Rayleigh-Plateau stability limit, thus Rayleigh-Plateau instability is not a concern here. 


\subsubsection{Simulation of capillary bridge formation in other engineered colloid}

assemblies

The self-stabilization mechanism (communicating vessels effect) makes the capillary bridges very tolerant of colloidal structure disorders as long as $\Phi_{A}$ is well below $\Phi_{A}{ }^{*}$ to avoid coalescence of neighboring capillary bridges. Such a mechanism is also able to stabilize capillary bridges in other compact colloid assemblies. The essential condition for the self-stabilization mechanism is that the internal pressure is a monotonically increasing function of the bridge volume, hence the particles are not restricted to be identical spheres. Even if there exist different particle geometries, ${ }^{78}$ as long as the Laplace pressure falls into a common range, equilibrium can also be achieved among the capillary bridges. Such behaviors are further demonstrated in Figures 3.18 and 3.19. Figure 3.18 shows the simulated capillary bridges formed between neighboring colloidal particles in a Saturn-ring-like superstructure. ${ }^{126}$ The center sphere has different radius, thus the final stable capillary bridges assume two different sizes. Just as in colloidal crystals, the capillary bridges in this microsphere assembly solely increase normal contact stress between the particles, thus the microstructure and symmetry of the assembly are well maintained. Figure 3.19 shows the simulated capillary bridges formed in an interstitial colloidal crystal consisting of spheres of two different sizes, where stable uniform capillary bridges are also obtained. In all these cases, the capillary bridges reinforce the otherwise fragile colloidal structures. 


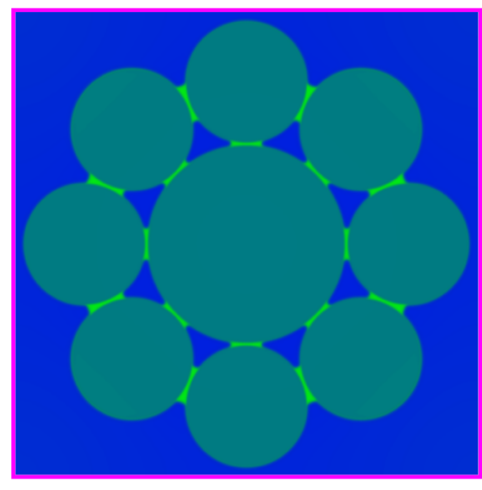

Time step $=10,000$

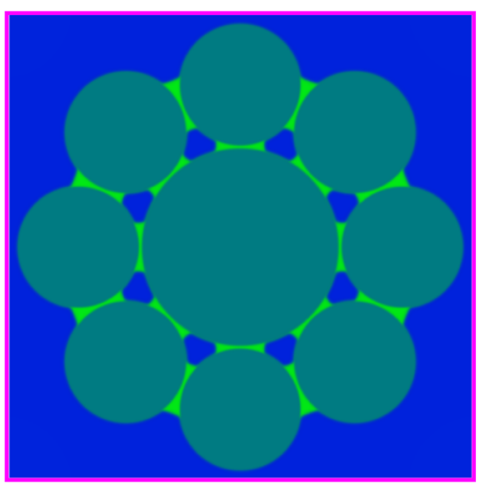

Time step $=50,000$

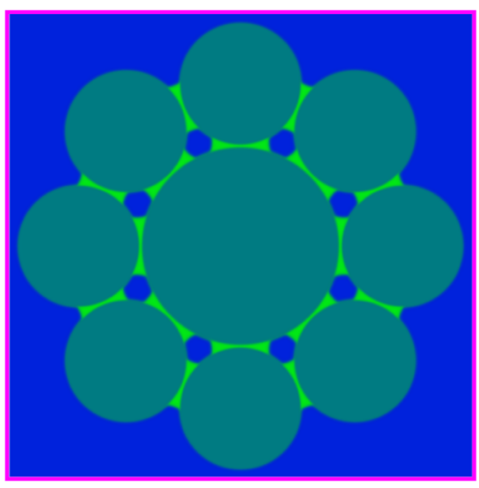

Time step $=800,000$

Figure 3.18 Simulated capillary bridge formation and self-stabilization in saturn-ring-like colloidal superstructure. The radii of the center and satellite spheres are $112 l$ and $70 l$, respectively. $528 \times 528$ mesh is used. $\Phi_{A}=0.07$.

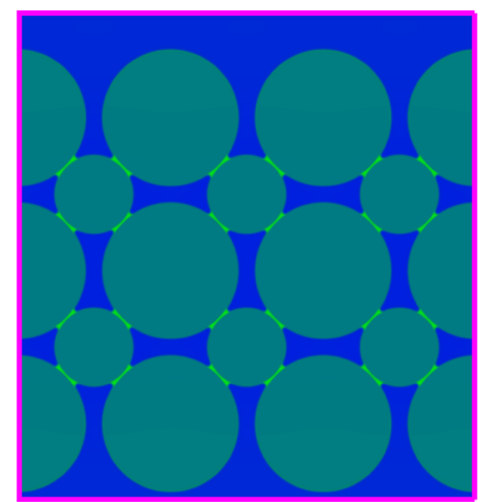

Time step $=10,000$

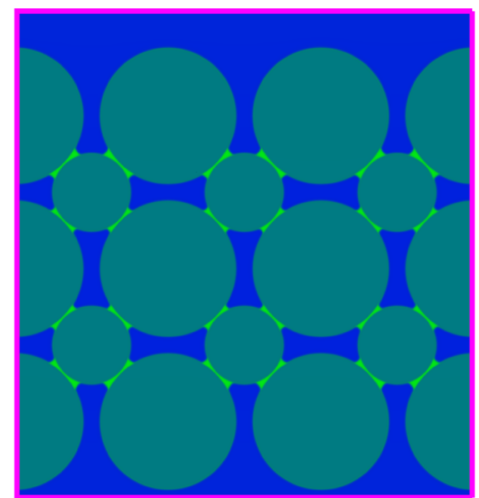

Time step $=50,000$

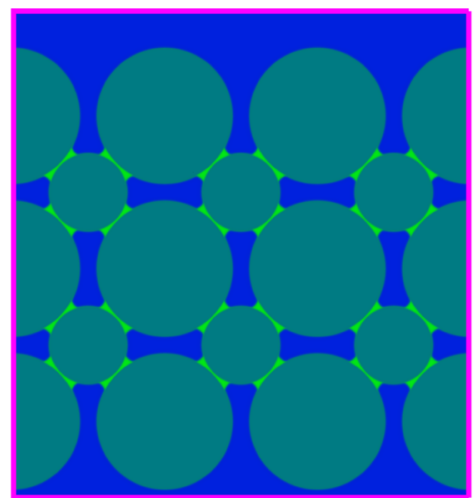

Time step $=800,000$

Figure 3.19 Simulated capillary bridge formation and self-stabilization in an interstitial colloidal crystal. The radii of different-sized spheres are $112 l$ and $70 l$, respectively. $\Phi_{A}=0.07 .768 \times 816$ mesh is used.

\section{Concluding Remarks}

Computer simulations of capillary bridge formation in various closely packed colloidal assemblies through liquid phase separation reveal a self-stabilization 
mechanism that operates through diffusive equilibrium of the two-phase liquid morphologies. Such mechanism renders desired microstructural stability and uniformity to the capillary bridges, which can be used to reinforce the colloidal microstructures with sufficient adhesion strength. This study sheds light on a promising route to in-situ (inliquid) firming of fragile colloidal crystals and other compact colloidal microstructures via spontaneous formation of uniform and stable capillary bridges through liquid phase separation, where the capillary bridges bond colloidal particles as mortar bonds bricks.

\subsection{Additional discussion about lateral capillary interaction}

\section{Two particles subject to external force at curved liquid interfaces: Comparison with flotation force}

In the previous cases, particles are not subject to any external force. With external force applied, the liquid interfaces could be deformed and induce lateral capillary interaction between particles. Figure 3.20 shows simulated motion of two spherical particles initial adsorbed in a sessile drop. As described above, without external force, they do not move or disturb the liquid interface. By setting which as initial state, we apply downward gravitational force to them and two extreme cases are presented. In Figure 3.20a, the gravitational force is small. As a result, the deformation of liquid interface is minor, and the particles slip down toward the bottom line of the sessile drop as if they are on a surface of a rigid hemisphere. Laterally they thus exhibit "repulsive" motion. However, if the gravitational force is large, as shown in Figure 3.20b, the liquid interface is severely deformed. When the particles fall down to a certain degree, the net horizontal capillary force acting on the particle is toward the symmetry axis of the drop. As a result, the particles exhibit attractive motion laterally, until they are close enough so that the short range steric repulsion takes effect and keeps their relative distance. 

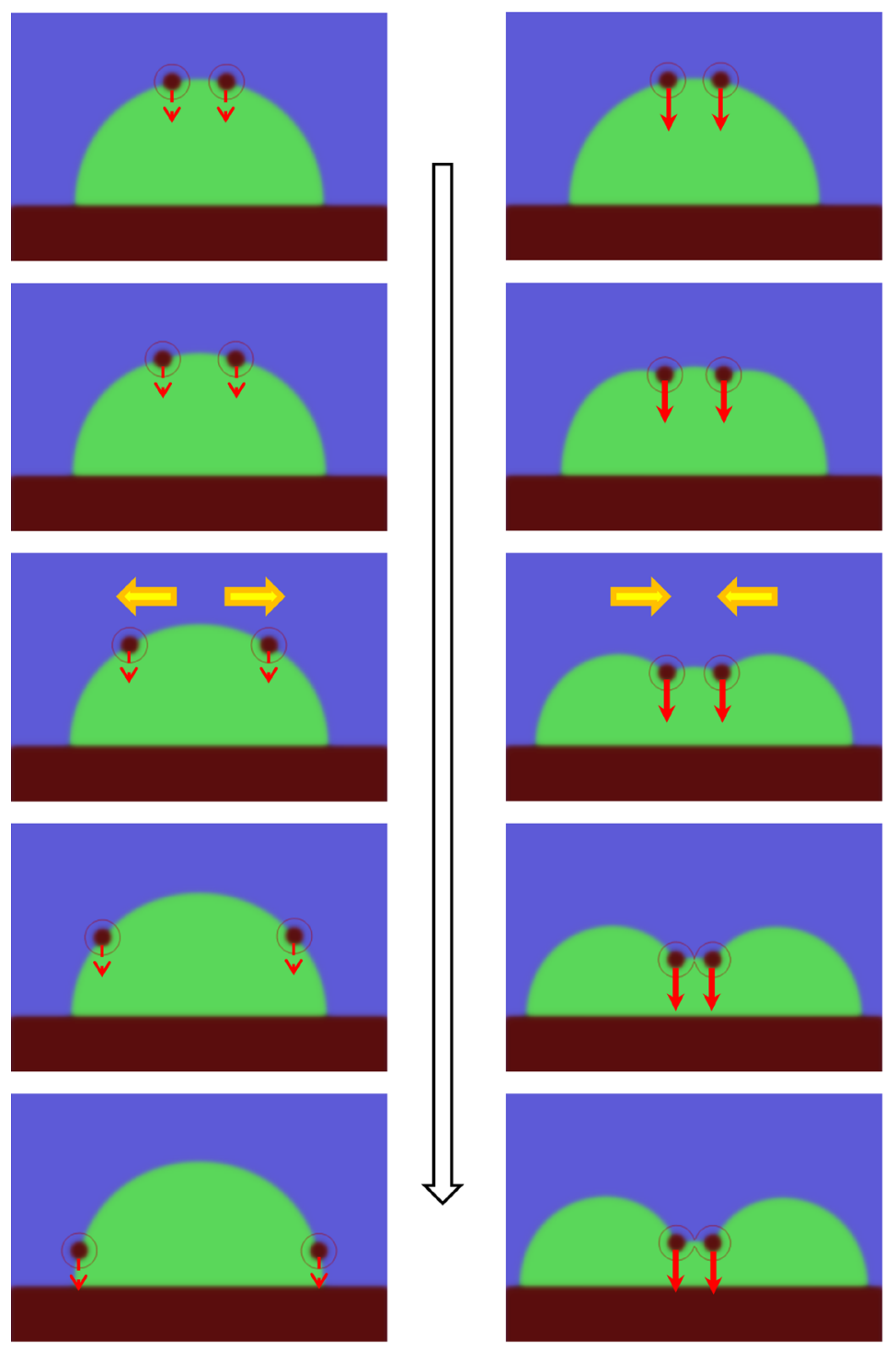

(a) $g=0.2 \gamma l$

(b) $g=2 \gamma l$

Figure 3.20 Simulated motion of two particles subject to external force (such as gravity) at the surface of a sessile drop. (a) Small gravity: particles slip down and appear to be repulsive to each other. (b) Large gravity: liquid interface is severely deformed which leads to attractive lateral capillary forces 
This behavior is reminiscent of the well-known flotation force ${ }^{35,127}$, for which the particles are subject to gravitational force adsorbed on otherwise flat liquid interfaces. Our results show the difference of particles on a curved liquid interface, where the lateral capillary interaction is affected by interfacial tension as well as the Laplace pressure. Both affect the shape of liquid interface throughout the entire process. Nevertheless, in future the coupled hydrodynamics should be solved simultaneously to achieve a more quantitative study.

\subsection{Supplementary material}

\subsubsection{Calculation details of capillary bridges}

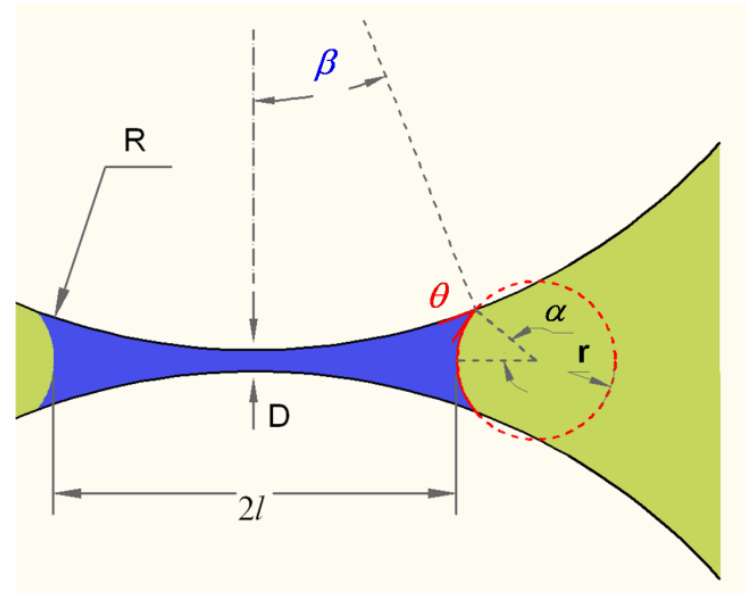

Figure 3.21 Definition of geometrical parameters for a meniscus in a slit between two spheres.

By adopting the circular approximation, the influence of geometrical confinement is straightforward. In order to maintain correct contact angle, the radius of meridional curvature is restricted to be (see Figure 3.21 for the definition of geometrical parameters)

$$
r=\frac{2 R(1-\cos \beta)+D}{2 \cos (\theta+\beta)}
$$


and the azimuthal radius is

$$
l=R \sin \beta-r(1-\cos \alpha),
$$

with $\alpha=\frac{\pi}{2}-(\beta+\theta)$ (shown in Figure 3.21) defined for convenience.

The pressure inside the meniscus can be thus calculated according to the YoungLaplace equation

$$
p=\gamma_{A B}\left(\frac{1}{l}-\frac{1}{r}\right) .
$$

The volume of a capillary bridge can be calculated as

$$
V^{c b}=V^{r o t}-2 V^{c a p},
$$

where

$$
V^{r o t}=2 \pi\left[r(l+r)^{2} \sin \alpha-r^{2}(l+r)(\sin 2 \alpha+2 \alpha) / 2+r^{3}\left(\sin \alpha-\sin ^{3} \alpha / 3\right)\right],
$$

and

$$
V^{c a p}=\frac{\pi}{3} R^{3}(2+\cos \beta)(1-\cos \beta)^{2} .
$$

The relation between pressure and the volume of capillary bridge is thus obtained from Eqs. (3.10)-(3.15). Instead of solving for an explicit function, it is easier to obtain the relationship curve as a parametric function through the parameter $\beta$ (plotted by Matlab). In Figure 3.13b, we set $D=0$, while the same trend of monotonic increase is found for nonzero $D$, as shown in Figure 3.22. Also, the volume of a capillary bridge corresponding to different filling angles can be plotted (as shown in Figure 3.23). The interfacial areas between particle/phase A $S_{A P}$ and phase-A/phase-B $S_{A B}$ can also be obtained by

$$
\begin{aligned}
& S_{A B}=4 \pi r[l \alpha+r(\alpha-\sin \alpha)] . \\
& S_{A P}=4 \pi R^{2}(1-\cos \beta)
\end{aligned} .
$$

The interface energy associated with an individual capillary bridge is

$$
\Delta E^{c b}=S_{A B} \gamma_{A B}+S_{A P}\left(\gamma_{A P}-\gamma_{B P}\right)=\left(S_{A B}-S_{A P} \cos \theta\right) \gamma_{A B},
$$

so the dependence of $\Delta E^{c b}$ on the volume $V^{c b}$ can be calculated (Figure 3.17). 


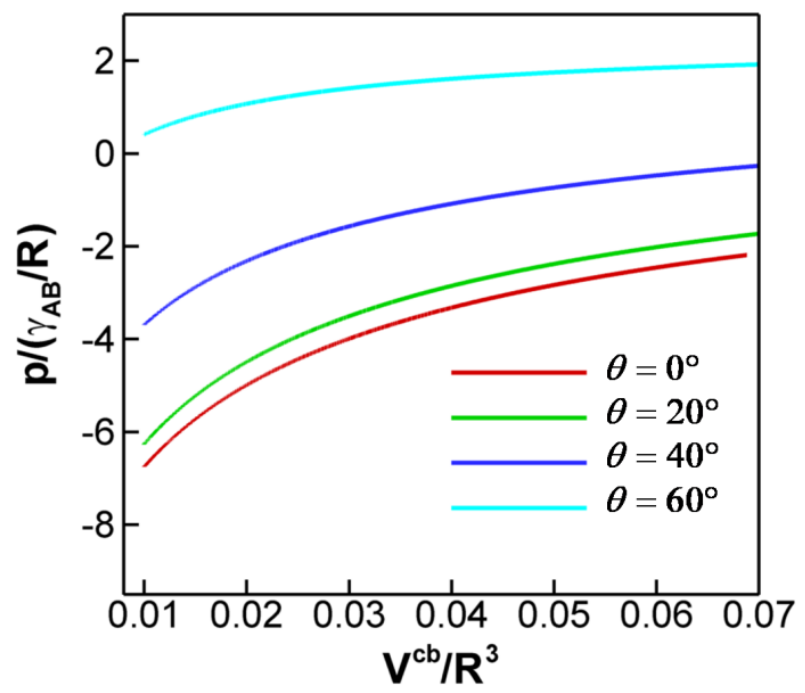

Figure 3.22 For acute contact angles, Laplace pressure still monotonically increases with increasing volume of a capillary bridge for small nonzero gap distance $(D=0.02 R)$.

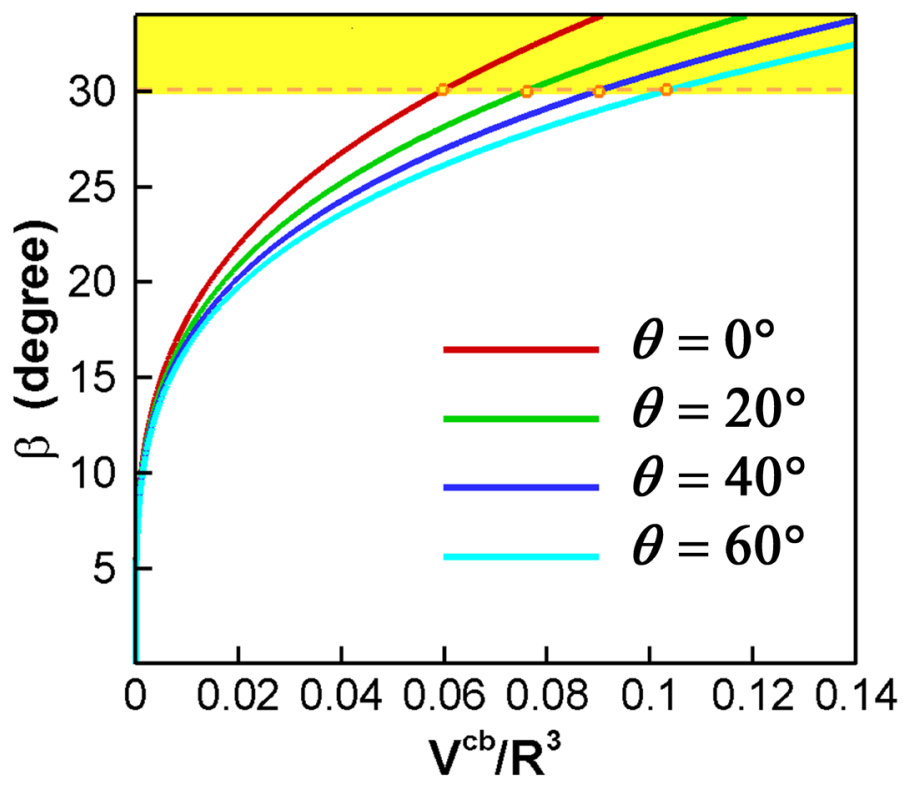

Figure 3.23 Dependence of filling angle $\beta$ on capillary bridge volume at different contact angles. Yellow zone indicates coalescence of neighboring menisci for fcc or hcp crystals. 
In two-dimensional cases, the calculation is much easier. Following the above approach, the pressure-volume relationship is obtained and shown in Figure 3.24, with the same trend as in $3 \mathrm{D}$ case.

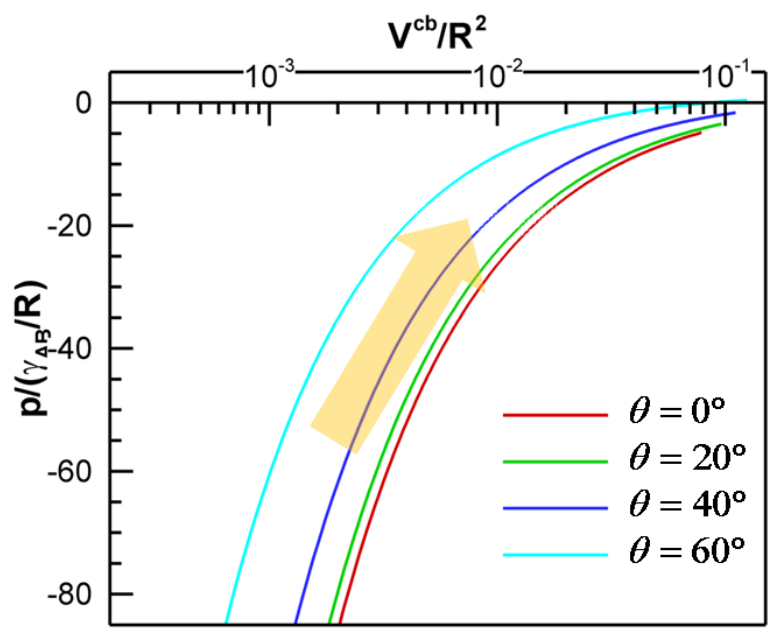

Figure 3.24 The Laplace pressure of a capillary bridge (2D) is also a monotonically increasing function of the capillary bridge volume, $V^{c b}$ (for cases of acute contact angle, well smaller than $90^{\circ}$ ).

\subsubsection{Calculation details of particles jamming at liquid interfaces}

\section{Positive pressure case:}

By the law of sines

$$
\frac{\overline{A C}}{\sin \theta}=\frac{l}{\sin \angle B A C}
$$

Since

$$
\angle A C O=\pi-\omega-\angle O A C=\pi-\omega-(\alpha-\angle B A C)
$$

and

$$
\frac{R}{\sin \angle A C O}=\frac{\overline{A C}}{\sin \angle A O C}=\frac{L}{\sin \omega}
$$


where $L=\overline{A C}=\sqrt{r^{2}+l^{2}-2 r l \cos \theta}$. The above equations still lead to

$$
\alpha=-\omega+\sin ^{-1}\left(\frac{R \sin \omega}{L}\right)+\sin ^{-1}\left(\frac{l \sin \theta}{L}\right)
$$

If the interparticle repulsive force is $F$ (with direction along the centers of the neighboring particles), the radial force component of each particle contributed from Laplace pressure is $2\left(\frac{\gamma}{l}\right) r \sin \alpha$, from interfacial tension is $-2 \gamma \sin (\theta+\alpha)$, and from steric repulsion is $2 F \sin \omega$. Therefore the force balance requires that

$$
\gamma\left[\frac{r}{l} \sin \alpha-\sin (\theta+\alpha)\right]+F \sin \omega=0
$$

which leads to

$$
F=\frac{\gamma}{\sin \omega}\left[\sin (\theta+\alpha)-\frac{r}{l} \sin \alpha\right]
$$

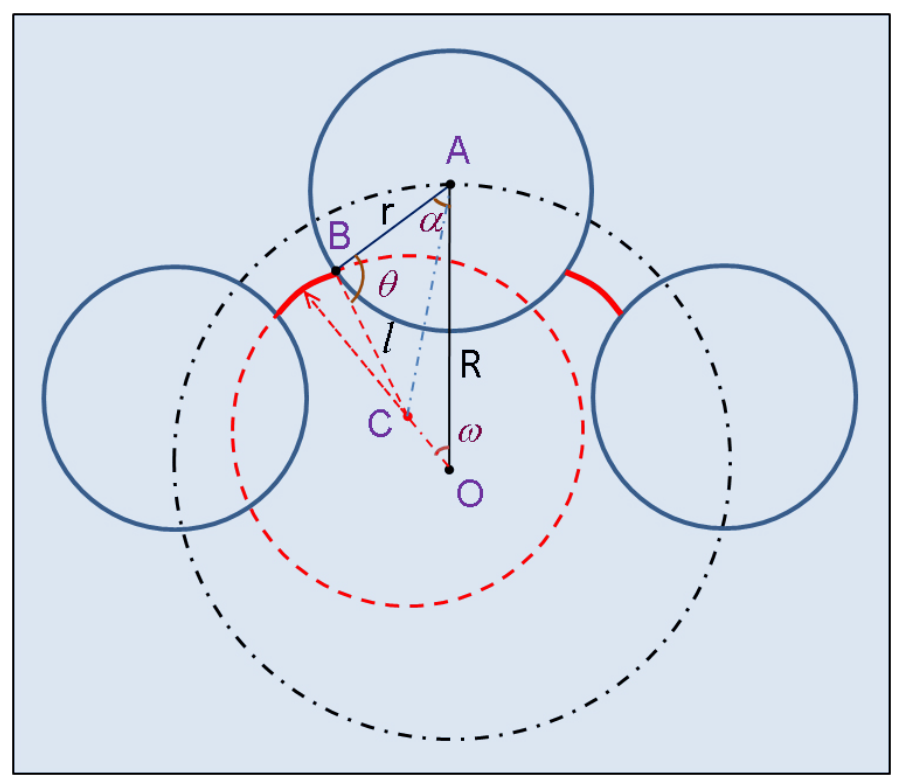

Figure 3.25 Definition of geometrical parameters for a particles jamming at liquid-liquid interfaces. Red solid lines stand for liquid-liquid interfaces. The actual radius of curvature is smaller than the apparent radius of curvature outlined by particle centers. 


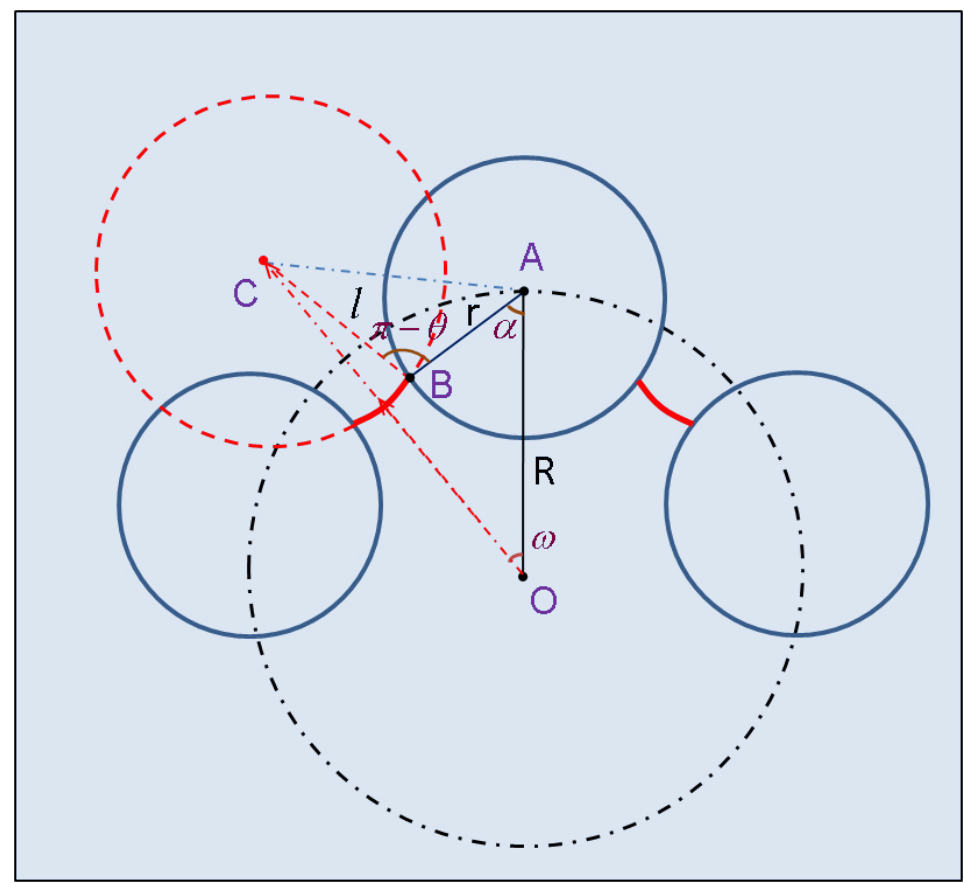

Figure 3.26 Definition of geometrical parameters for a particles jamming at liquid-liquid interfaces. Red solid lines stand for liquid-liquid interfaces. The curvature is negative.

\section{Negative pressure case (imaginary, Figure 3.26):}

By the law of sines

$$
\frac{\overline{A C}}{\sin (\pi-\theta)}=\frac{l}{\sin \angle B A C}=\frac{L}{\sin \theta}
$$

Since

$$
\angle A C O=\pi-\omega-\angle O A C=\pi-\omega-(\alpha+\angle B A C)
$$

and

$$
\frac{R}{\sin \angle A C O}=\frac{\overline{A C}}{\sin \angle A O C}=\frac{L}{\sin \omega}
$$

where $L=\overline{A C}=\sqrt{r^{2}+l^{2}+2 r l \cos \theta}$. The above equations still lead to 


$$
\alpha=-\omega+\sin ^{-1}\left(\frac{R \sin \omega}{L}\right)-\sin ^{-1}\left(\frac{l \sin \theta}{L}\right)
$$

If the interparticle repulsive force is $F$ (with direction along the centers of the neighboring particles), the radial force component of each particle contributed from Laplace pressure is $-2\left(\frac{\gamma}{l}\right) r \sin \alpha$, from interfacial tension is $-2 \gamma \sin (\theta+\alpha)$, and from steric repulsion is $2 F \sin \omega$. Therefore the force balance requires that

$$
\gamma\left[-\frac{r}{l} \sin \alpha-\sin (\theta+\alpha)\right]+F \sin \omega=0,
$$

which leads to

$$
F=\frac{\gamma}{\sin \omega}\left[\sin (\theta+\alpha)+\frac{r}{l} \sin \alpha\right]
$$




\section{Chapter 4. Self-assembly of colloids with heterogeneous charges and dipoles}

DIFA employs field variables to depict in detail the charge distribution on surface as well as in body of particles instead of point dipole approximation on the whole particle. In this chapter, the charge heterogeneity effect is studied. The simulation results verify and demonstrate the capability of our numerical model. The carrier fluid is assumed to be deionized, therefore charge transport is not included. Self-assembly and alignment of particles with magnetic dipoles under external magnetic field is also investigated. The simulation results demonstrate a route to computer aided design of magnetic composites.

The modeling algorithms have been described in chapter 2 .

\subsection{Aggregation of oppositely charged particles}

In atomic level ionic system, charge neutrality plays a dominant role in determining the stoichiometry and thus the composition of the ionic crystal or some covalent molecules. This can be different in colloid system. Leunissen et al shown that in colloidal crystal the stoichiometry was not dictated by charge neutrality, in contrast to atomic system. ${ }^{9}$ The reason is two-fold. The first reason is that in colloid system net charge tends to be screened by electric double layer, while the latter has a potential decays exponentially. In additional to the screening effect, in atomic system the electrostatic energy caused by net charge is huge compared with the thermal energy, characterized by $k_{B} T$ (Here we take room temperature) thus molecules with net charge cannot exist virtually. However, for a colloidal particle the average interparticle distance is much greater than atomic size therefore the electrostatic interaction is significantly weakened. Here through computer simulation we show the aggregation of oppositely charged particles despite the net charge. 
In this chapter, the carrier liquid is monophase, thus no interfacial tension exists. The energy here is thus normalized by the thermal energy $k_{B} T$ instead of interfacial tension. The normalization details of related parameters are listed in Table 4.1.
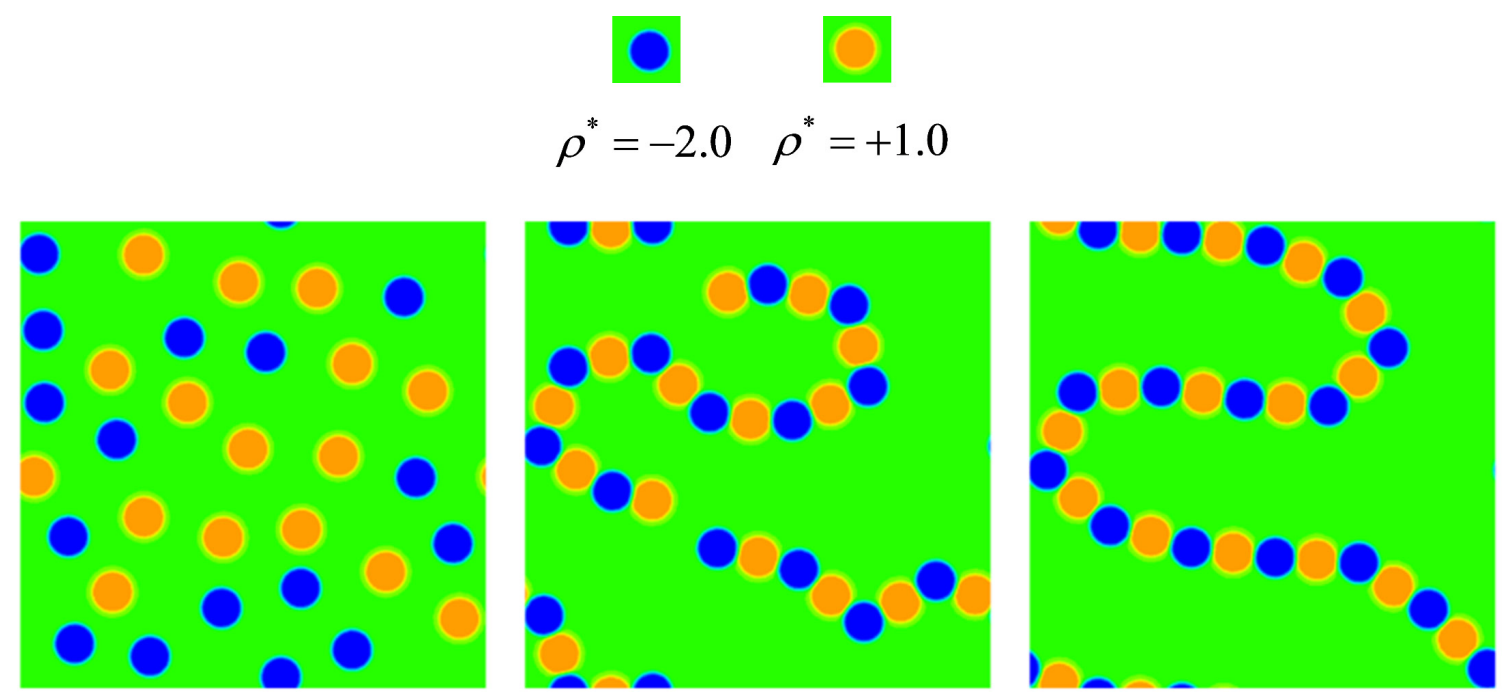

Figure 4.1 Simulation results show self-assembly of oppositely charged particles with equal number of particles $\left(N_{+}: N_{-}=1: 1\right)$ but unequal charge density $\left(\rho_{+}: \rho_{-}=1: 2\right)$. Particles aggregate to form long chain despite partial charges. The mesh size of the system is $256 \times 256$, and particle radius is 10 .

Figure 4.1 shows simulation of aggregation of randomly distributed particles with opposite charges. The orange particles each take charge 1 and the blue particles each take -2 . Without external field, the particles spontaneously aggregate to form short chains and then a long chain. The reason why a short chain still attracts another one appears unreasonable, considering each short chain takes net negative charge. The answer is that the attractive dipole-dipole interaction may dominate over repulsive net charge interaction at small distance. This deduction is verified by a further simulation in which the system size is increased from $256 \times 256$ to $512 \times 512$ with the same particles. In other word, initially the average interparticle distance is broadened. As a result, in Figure 4.2 it is found that initially particles still aggregate due to electrostatic interaction as well as 
Brownian motion. Whereas, when the particles aggregate into small clusters, they begin to repel each other and coexist, rejecting further agglomeration.

Table 4.1

Normalization of parameters generally used in simulations in Chapter 4

\begin{tabular}{|c|c|c|c|c|}
\hline Parameters & $\begin{array}{c}\text { Characteri } \\
\text { stic } \\
\text { Unit }\end{array}$ & $\begin{array}{l}\quad \text { Red } \\
\text { uced } \\
\text { value } \\
\text { used } \\
\end{array}$ & SI unit & $\begin{array}{l}\text { Magnitude of } \\
\text { characteristic } \\
\text { unit }\end{array}$ \\
\hline Grid size & $l_{0}$ & 1 & {$[\mathrm{~m}]$} & $10^{-7}$ \\
\hline \begin{tabular}{l}
\multicolumn{1}{r}{ Particle } \\
translation \\
mobility
\end{tabular} & $M_{0}$ & $\sim 0.1$ & $\begin{array}{r}{[\mathrm{N}]^{-}} \\
{ }^{1}[\mathrm{~m}][\mathrm{s}]^{-1}\end{array}$ & $10^{6}$ \\
\hline $\begin{array}{l}\text { Rotation } \\
\text { mobility }\end{array}$ & $N_{0}=M_{0} / l_{0}^{2}$ & $\begin{array}{l}\sim 0.0 \\
01\end{array}$ & $\begin{array}{l}{[\mathrm{N}]^{-1}[\mathrm{~m}]^{-}} \\
{ }^{2}[\mathrm{~s}]^{-1}\end{array}$ & $10^{20}$ \\
\hline Time step & $l_{0}^{2}\left(M_{0} k_{B} T\right)^{-1}$ & $\sim 0.1$ & {$[\mathrm{~s}]$} & $10^{-4}$ \\
\hline Charge & $q_{0}=\sqrt{\varepsilon_{0} k_{B} T l_{0}}$ & $\sim 0.1$ & {$[\mathrm{C}]$} & $10^{-19}$ \\
\hline Electric field & $E_{0}=k_{B} T q_{0}^{-1}$ & & {$[\mathrm{~V}][\mathrm{m}]^{-1}$} & $10^{-2}$ \\
\hline $\begin{array}{l}\text { Rigid body } \\
\text { coeff. }\end{array}$ & $k_{B} T l_{0}^{-3}$ & $\sim 25$ & {$[\mathrm{~Pa}]$} & 1 \\
\hline $\begin{array}{ll} & \text { Magnetizatio } \\
\end{array}$ & $\sqrt{k_{B} T / \mu_{0} l_{0}{ }^{3}}$ & $\sim 1$ & {$[\mathrm{~A}][\mathrm{m}]^{-1}$} & $10^{3}$ \\
\hline Energy & $k_{B} T$ & $\sim 1$ & {$[\mathrm{~J}]$} & $10^{-21}$ \\
\hline Force & $F_{0}=k_{B} T l_{0}^{-1}$ & & {$[\mathrm{~N}]$} & $10^{-14}$ \\
\hline
\end{tabular}

\subsection{Aggregation of patchy particles}

In previous work, particles are with uniform charge density, while actually even the particle body is homogeneous, the surface properties can be heterogeneous. Chen et al attribute the surface heterogeneity of polystyrene sphere to the surface phase separation into hydrophilic components and hydrophobic components. ${ }^{39}$ First we use a simple 
analytical model to show the basic characteristics of electrostatic interaction between two heterogeneously charged particles.
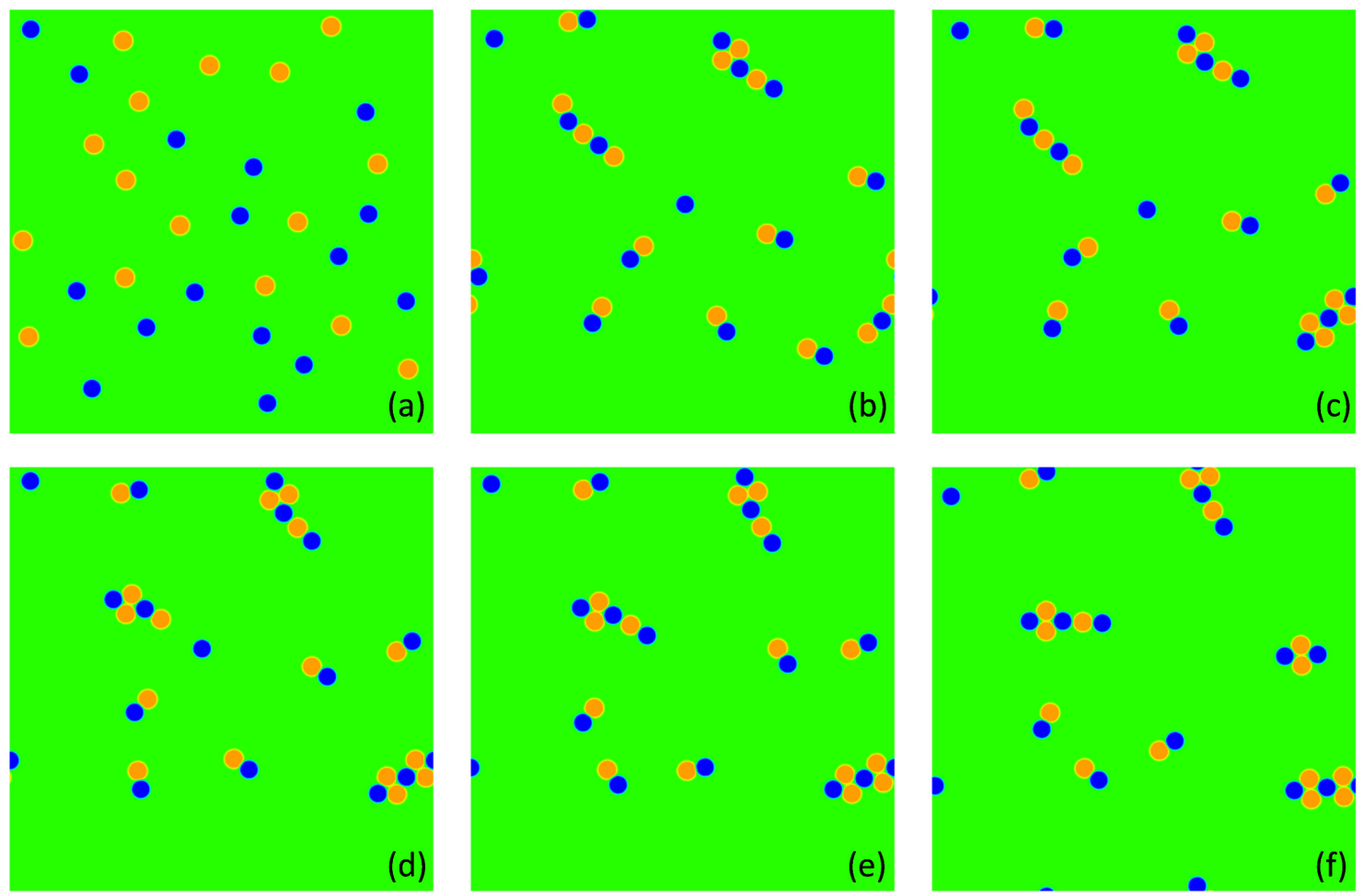

Figure 4.2 Simulation results show self-assembly of oppositely charged particles: discrete clusters are formed $\left(N_{+}: N_{-}=1: 1, \rho_{+}: \rho_{-}=1: 2\right.$ and particle radius is also 10, which is the same as in the previous figure). The difference is that the mesh size is $512 \times 512$, thus mean interparticle distance is increased.

The electrostatic force between the two circular patchy particles (in 2D, see Figure $4.3)$ is given by

$$
f^{e l-2 D}=\frac{1}{2 \pi \varepsilon} \frac{q^{2}}{D}\left[\frac{\left(1+Q^{*}\right)^{2}+1}{r^{*}}-\frac{2 r^{*}\left(1+Q^{*}\right)}{\left(r^{*}\right)^{2}-1}\right]
$$


where $r^{*}=r / D$ and $Q^{*}=Q / q$ are nondimensional particle distance and net charge. In more general $3 \mathrm{D}$ case, it is

$$
f^{e l-3 D}=\frac{1}{4 \pi \varepsilon} \frac{q^{2}}{D^{2}}\left\{\frac{\left(1+Q^{*}\right)^{2}+1}{\left(r^{*}\right)^{2}}-\frac{2\left[\left(r^{*}\right)^{2}+1\right]\left(1+Q^{*}\right)}{\left[\left(r^{*}\right)^{2}-1\right]^{2}}\right\}
$$

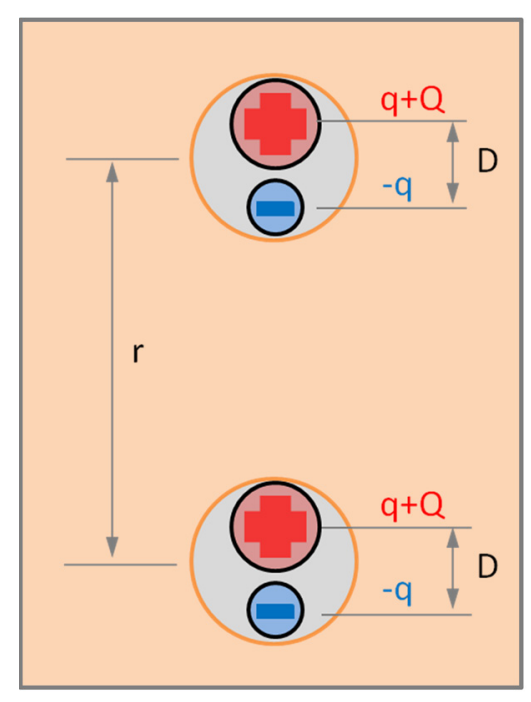

Figure 4.3 Schematic of two particles with patchy charges (can be viewed as with dipoles as well as net charges)

The total electrostatic force between the two patchy particles is mainly contributed from two parts. One is net charge repulsion and the other is dipole-dipole attraction. However, both terms may not be taken as point charge or point dipole for real particles of arbitrary geometry, especially when interparticle distance is comparable with particle size. Figure 4.4 and 4.5 show the total electrostatic force under 2D and 3D cases, respectively (The trends are similar). It is found that when interparticle distance is small or when the net charge is small the total force is attractive and repulsive otherwise. This is due to the fact that dipole-dipole interaction energy decays faster than Coulombic net charge interaction. The dashed black lines indicate the position where attractive interaction 
balances with repulsion and the total force is zero. $Q^{*}=Q / q$ is also a reflection of charge heterogeneity. The smaller $Q^{*}$ is, the more severe the heterogeneity is.

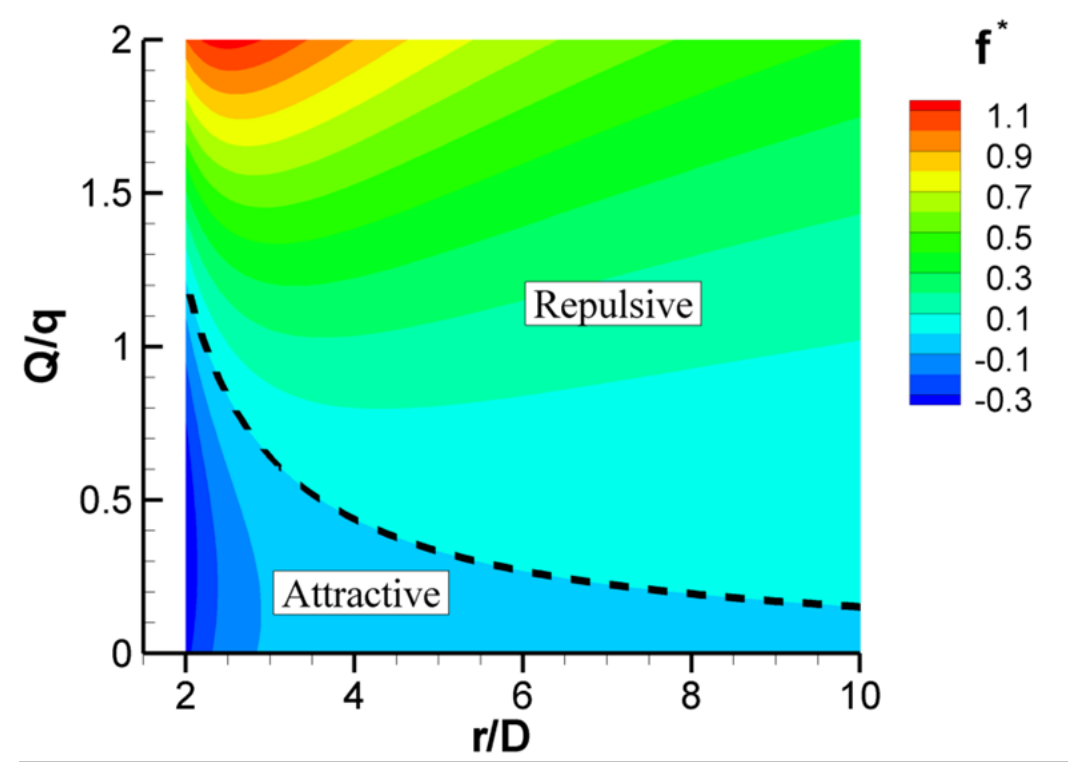

Figure 4.4 Distribution of electrostatic force between two patchy particles with heterocharges (two-dimensional case)

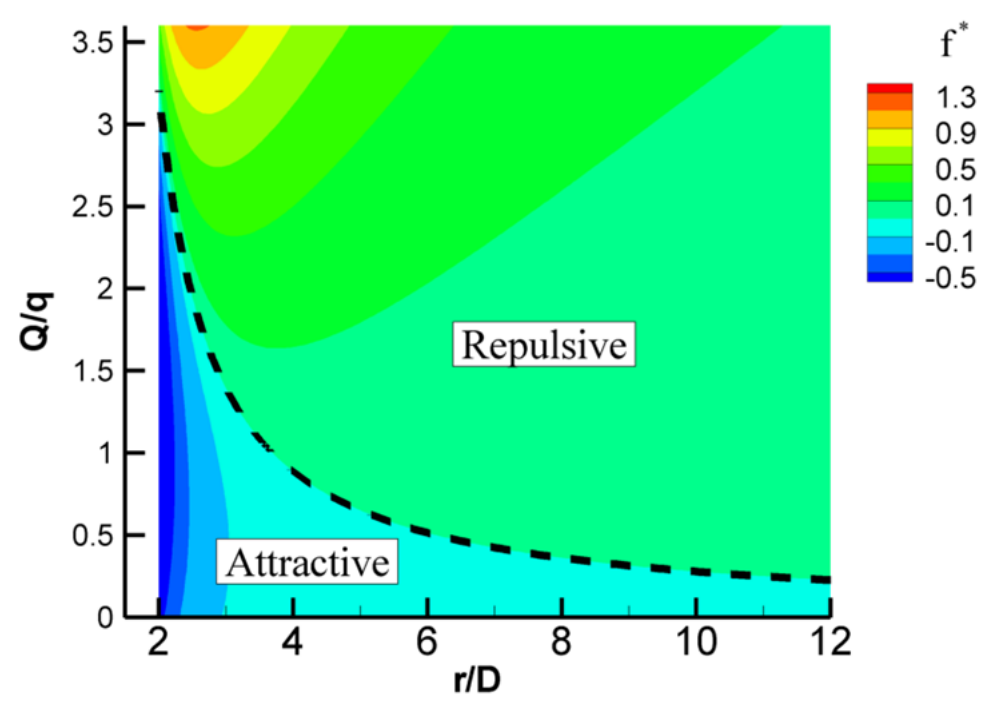

Figure 4.5 Distribution of electrostatic force between two patchy particles with heterocharges (three-dimensional case) 


\section{Formation of dimers}

Figure 4.6 shows simulation of the spontaneous aggregation process of colloidal particles with patchy charges. The parameters used are listed in Table 4.1. Each particle are divided into two patches and on one patch the charge is $-q^{*}=-30$, and the other $Q^{*}+q^{*}=45$. Therefore each particle carries net charge of $Q^{*}=15$. If we ignore the detail of patchy-style charge, all particles can be viewed to take "like-charge". Initial configuration of Figure 4.6(a) is distantly but randomly spaced particles relax their position under electrostatic force (Brownian forces intentionally removed). Due to likecharge repulsion (net charge), the particles are stabilized to be away from each other. With this initial configuration and Brownian motion applied, Figure 4.6 (b) shows that part of particles begin to aggregate to form dimers. The aggregation has to be attributed to dipole-dipole interaction, although the dipoles possess finite size here and thus are not point dipoles. Figure 4.7 shows the schematic of energy profile for two patchy particles as a function of interparticle distance (c.f. Figure 4.4 and 4.5). The thermal fluctuation energy allows the particles to have opportunity to overcome the repulsive energy barrier, $E^{r e p}$. Meantime if particles are attached together, they need energy of $E^{\text {att }}$ to jump out of the attractive well.

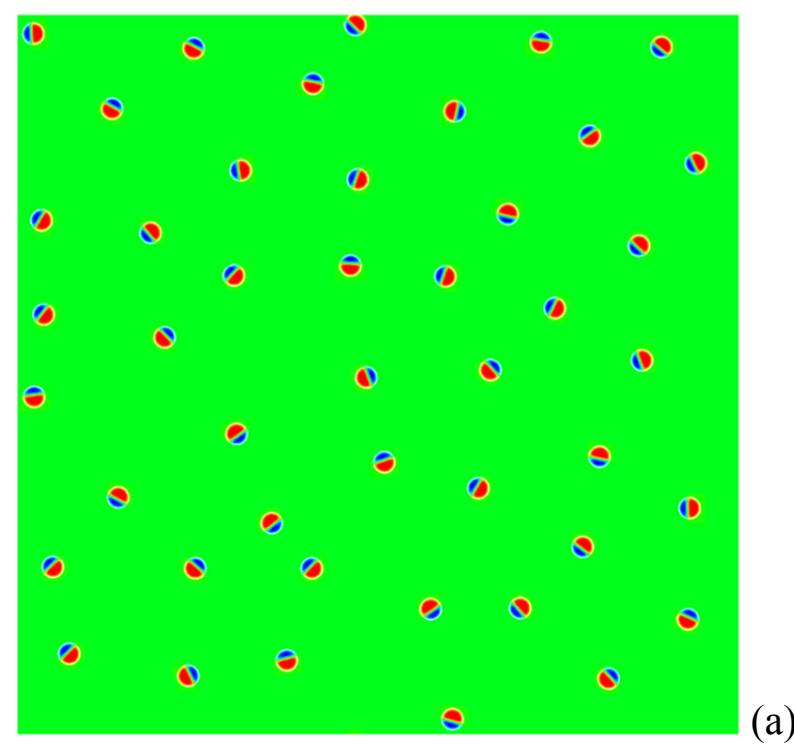



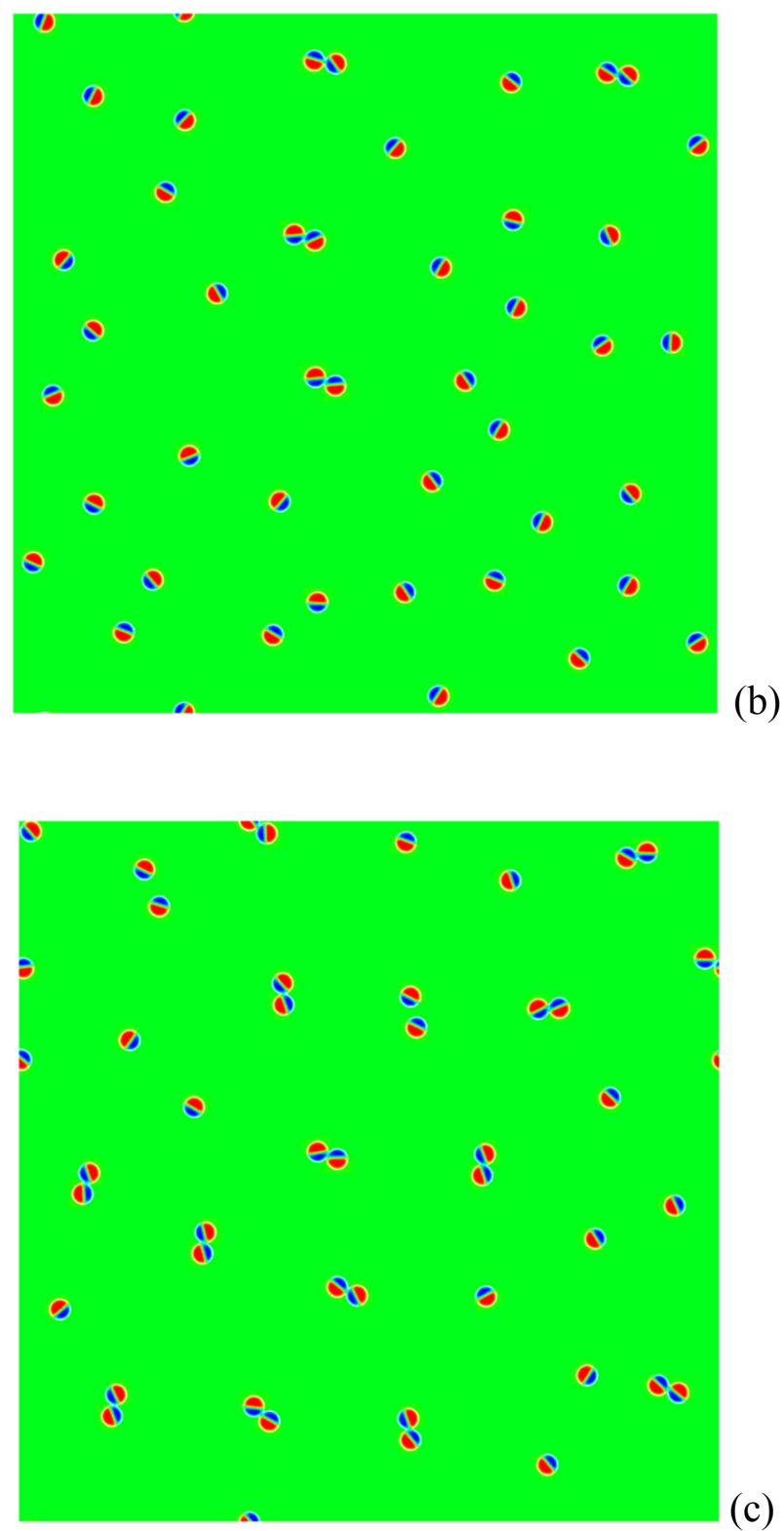

(c)

Figure 4.6 Simulation result (2D) shows aggregation of patchy particles under electrostatic interaction and Brownian motion, the particles form coexisting dimers finally. Here $Q^{*}=15, q^{*}=30$ and particle radius $a^{*}=10$. The normalized time corresponding to each state (a), (b), (c) is 0, 20,000 and 200,000, respectively. 


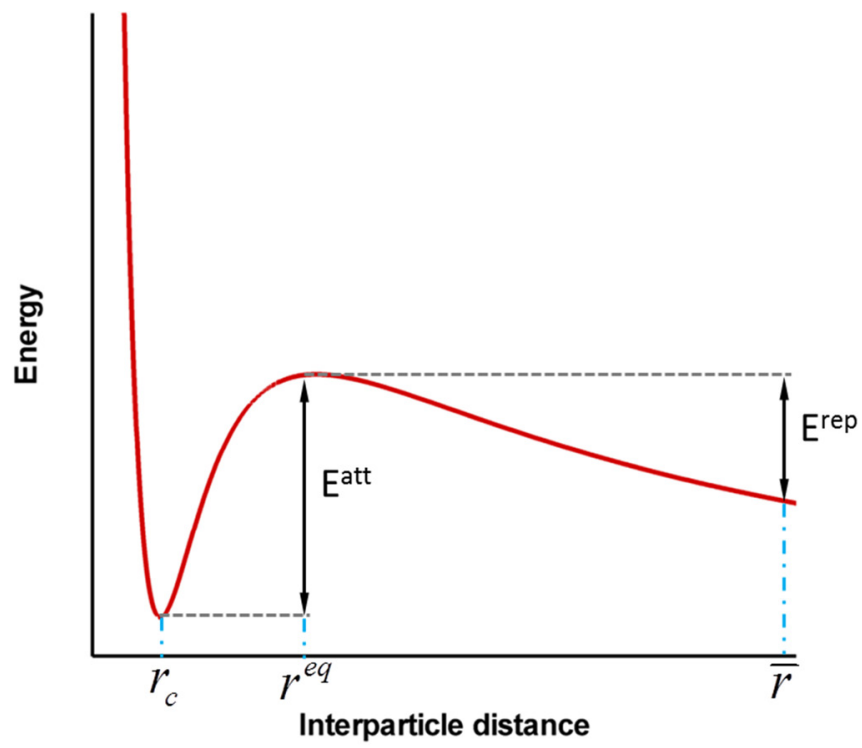

Figure 4.7 Schematic of electrostatic energy profile between two particles with patchy charges shown in Figure 4.3.

$E^{\text {rep }}$ determines the rate of particle association and $E^{\text {att }}$ the rate of particle disassociation. Thermodynamically whether the particles tend to aggregate or not thus depends on the ratio $E^{r e p} / E^{\text {att }}$. Figure 4.6 is an example that $E^{\text {rep }} / E^{\text {att }}$ is small so the dimers formed are stable.

It needs to be noted that when the two patchy particles are attached together. The energy minimized state is not that the two dipoles are perfectly aligned as shown in Figure 4.3. As shown in Figure 4.6(c), within the dimer the two dipoles are in opposite direction and misaligned, thus the dimers do not have net dipole term as a whole (The mass center of negative charges overlaps with that of positive charges, so only higher order multipole terms are present). This state has even lower free energy than the state the two dipoles are perfectly aligned. In other words, the energy calculation plotted in Figure 4.4 and 4.5 is overestimated for close distance. The quantitative energy barrier for any heterogeneously charged particles thus have to be numerically calculated. 
Each dimer carries net charge of $2 Q^{*}$. If we define a nondimensional length of

$$
\lambda_{B}=\frac{Q^{2}}{4 \pi \varepsilon k_{B} T}
$$

which is essentially the same as Bjerrum length for elementary charges, the increased net charge greatly elongates $\lambda_{B} . \lambda_{B}$ is the characteristic radius of a repelling zone where thermal energy is difficult to drive outside particles in. If $\lambda_{B}$ is large, the charged units will be difficult to approach each other to further aggregate.

At equilibrium the dimer appears to possess two-fold rotational symmetry. Since each dimer does not possess dipole any more as a whole, when two particles aggregate to form a dimer, the net charge is doubled, while the potential attractive dipole-dipole is absent (only thermal fluctuation induced dipole may be present which is much weaker). In total the electrostatic force between dimers and between dimer and monomer becomes strongly repulsive, thus the dimers are stable against Brownian motion and maintained distantly from each other and monomers, as shown in Figure 4.6c.

\section{Formation of trimers}

As has been stressed in the introduction chapter, electrostatic interaction between heterogeneously charged particles is highly configuration dependent. For the equilibrium configuration of the isolated dimer (Figure 4.6), it does not possess net dipole thus the dimers are stable and do not aggregate further. However, this is not always the case. When the third charge (or dipole) is approaching the dimer, it may alter the configuration

of the dimer and thus induce dipole, which is similar to the induction force between molecules. ${ }^{29}$ In order to allow the third particle join, the net charge repulsion effect should be relieved and dipole-dipole interaction should be enhanced. In Figure 4.8, we set $Q^{*}=15, q^{*}=60(Q / q$ is decreased and attraction increased, see Figure 4.5$)$ and observe 
formation of trimers. In Figure $4.8 \mathrm{~b}$, monomers begin to aggregate to form dimers and there are 2 trimers formed. Each trimer carries net charge of $3 Q^{*}$. The trimer possess three-fold rotation symmetry and it does not either have dipole as a whole. Therefore, the trimers are more difficult to approach than dimers for positive charge thus are more stable. When more and more trimers are formed, as shown in Figure 4.8c and Figure 4.8d, the monomers and dimers are more restricted and have smaller activity region (due to Brownian motion). As a result, the trimers are coexisting with dimers and monomers finally, as shown in Figure 4.8d.

\section{Monomers only}

From Figure 4.7, it can be concluded that if the attractive well $E^{\text {att }}$ is small compared with thermal energy, $k_{B} T$, then the coagulated particle complex (such as the dimer) will be unstable. Figure 4.9 is such a case where $Q^{*}=7, q^{*}=14$ thus the electrostatic energy is greatly reduced. As a result, the particles randomly collide but do not coagulate. In Figure $4.9 \mathrm{~b}$, it can be found that there is a dimer configuration (close to the middle-right of the image) but in Figure 4.9c, it disappeared. There are no dimers or trimers survive in Figure 4.9c.

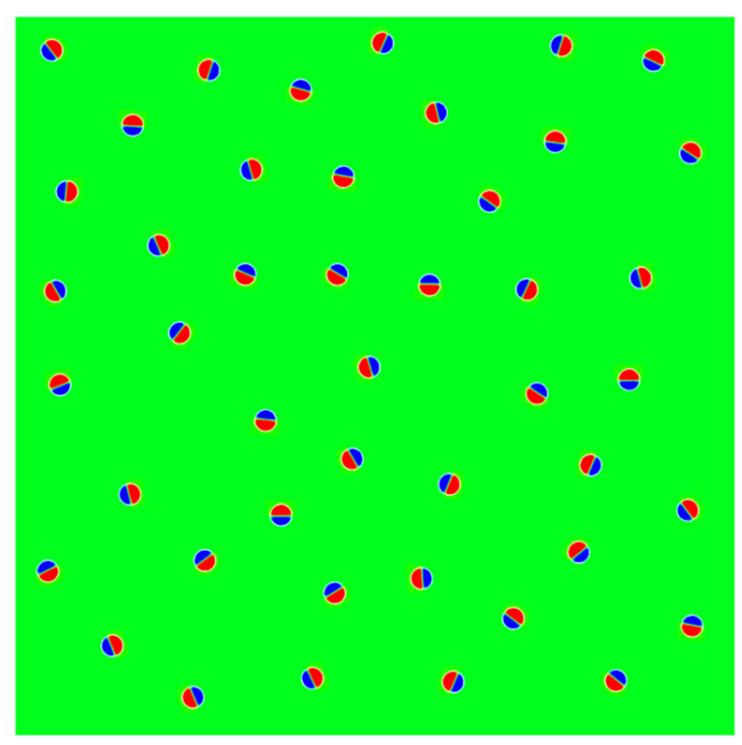

(a) 

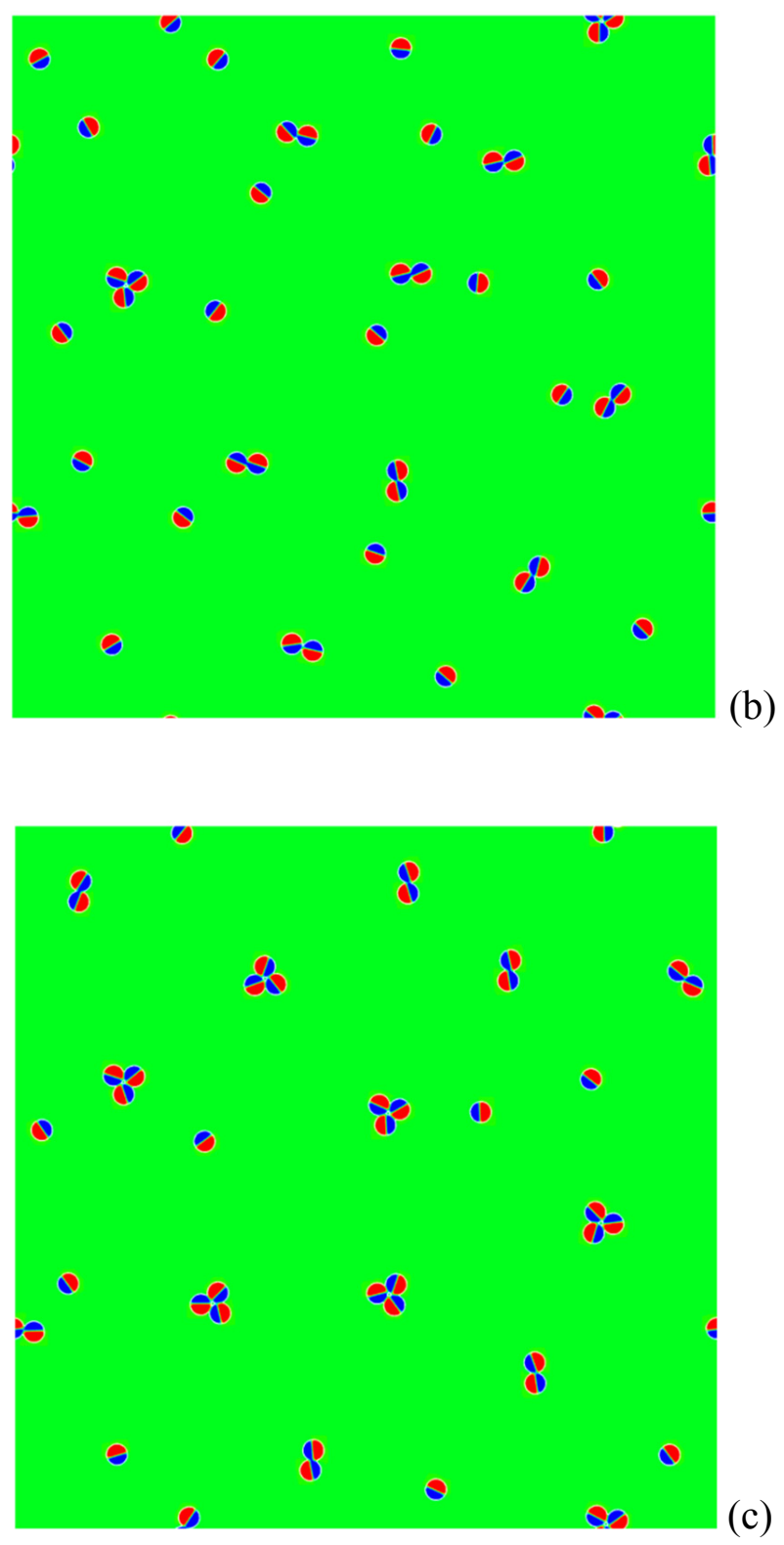


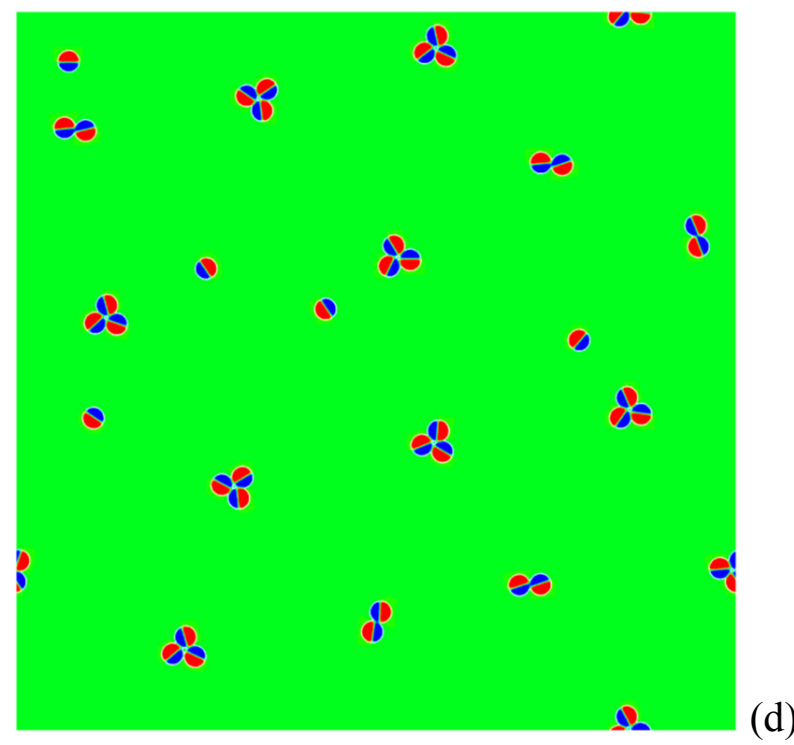

Figure 4.8 Simulation result (2D) shows aggregation of patchy particles under electrostatic interaction and Brownian motion, the particles form coexisting trimers, dimers and monomers finally. Here $Q^{*}=15, q^{*}=60$ and particle radius $a^{*}=10$. The normalized time corresponding to each state (a), (b), (c), (d) is 0, 20,000, 40,000 and 400,000 , respectively.

In summary, if $E^{\text {att }} \ll k_{B} T$, no aggregates are expected to survive; if $E^{r e p} \gg k_{B} T$, no aggregates are expected either since the thermal energy is unable to overcome the repulsive energy barrier; if $E^{\text {att }} \gg E^{\text {rep }} \sim k_{B} T$, the aggregates will be stable; if $E^{a t t} \sim E^{r e p} \sim k_{B} T$, the particle association rate and disassociation rate will be comparable, the particle aggregates will be in dynamic equilibrium and the final population of aggregates will depend on the ratio of $E^{\text {att }} / E^{\text {rep }}$. It needs to be noted that although electric double layer effect is not considered, this charge heterogeneity model already explains many phenomena about electrostatic interaction, such as apparent like-charge attraction, dynamic equilibrium of particle aggregates, and dependence of aggregate size on charge heterogeneity. The above analysis did not incorporate of electric double layer, thus it applies to the cases in which the Debye length is much larger than interparticle 
distance, and it is expected to provide insights and a possible explanation to the likecharge attraction phenomena.
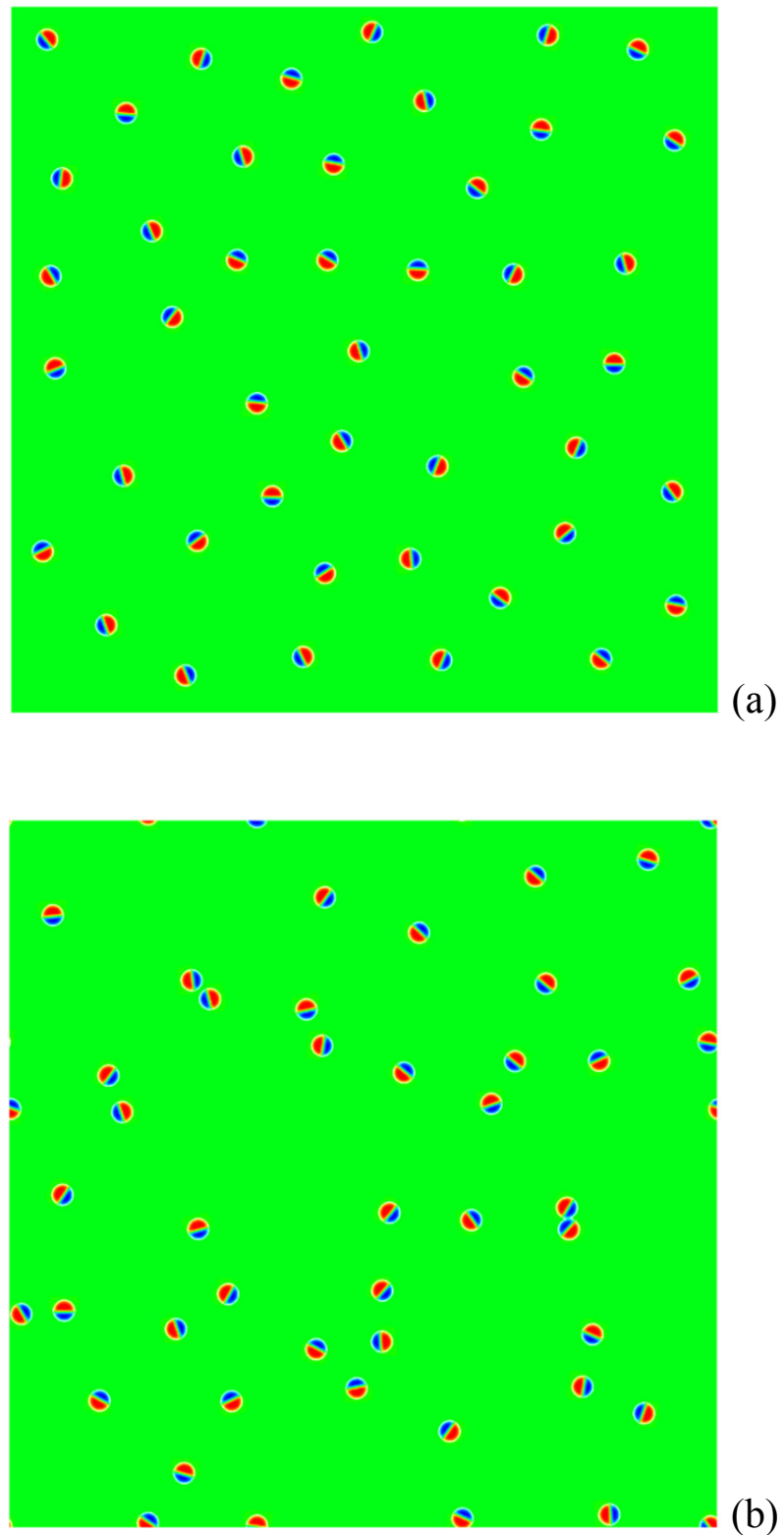

(b) 


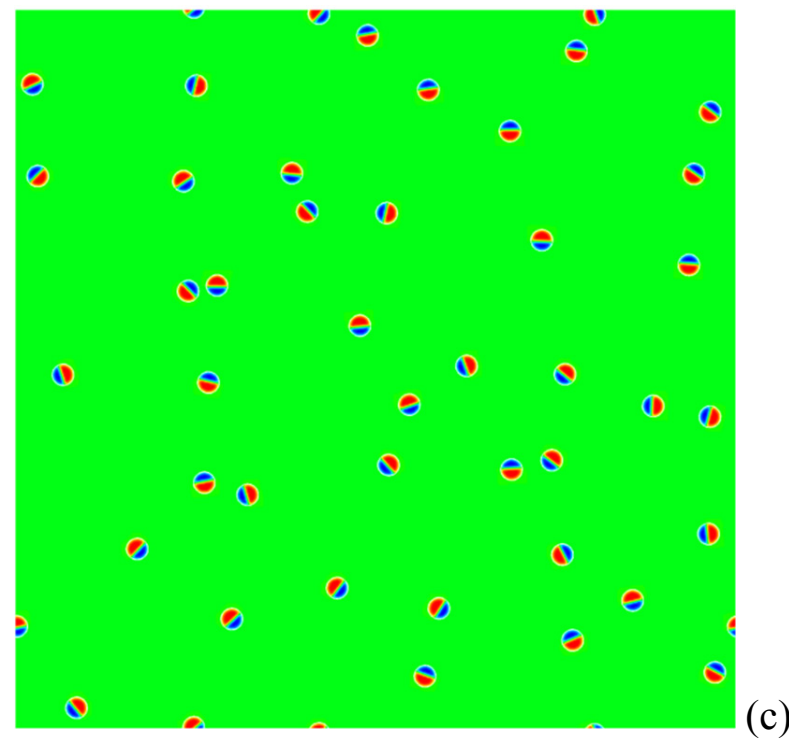

Figure 4.9 Simulation result (2D) shows aggregation of patchy particles under electrostatic interaction and Brownian motion. There are only monomers finally, i.e., no stable dimers or trimers survive. Here $Q^{*}=7, q^{*}=14$ and particle radius $a^{*}=10$. The normalized time corresponding to each state (a), (b), (c) is 0, 16,000 and 200,000, respectively.

\subsection{Formation of ionic colloidal crystal}

Colloids with long range attractive interaction (e.g., Coulombic interaction between oppositely charged particles) tend to irreversibly aggregate, ${ }^{128}$ without formation of ordered crystal-like microstructures. As has been demonstrated in previous sections, heterogeneously charged particles may aggregate due to attractive dipole-dipole interaction even the net charges on particles are with the same sign. However, charge neutrality is still corresponding to the energy minimization state. Thermal fluctuation energy must be able to compete with electrostatic energy so as to allow the system explore the full free energy landscape and avoid be trapped into the deep metastable heteroaggregation states. 


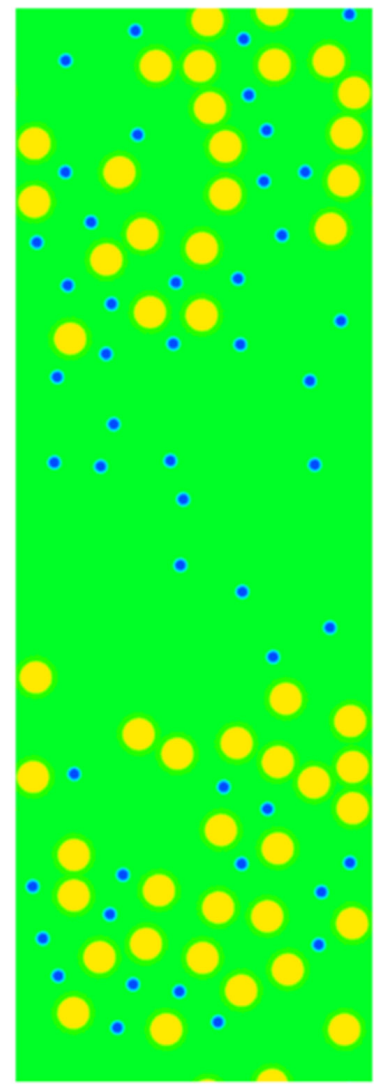

(a)

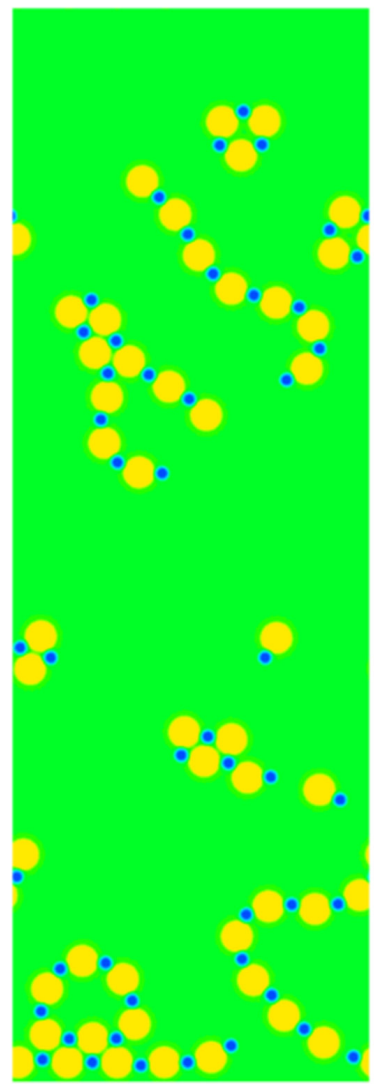

(b)

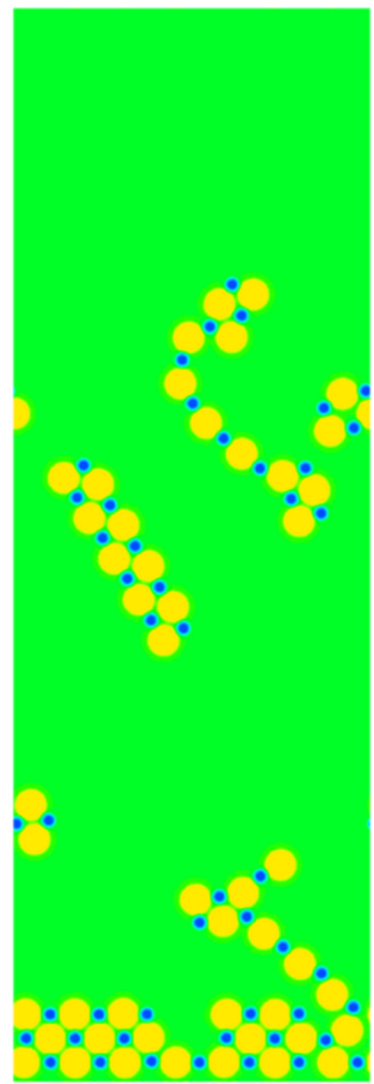

(c)

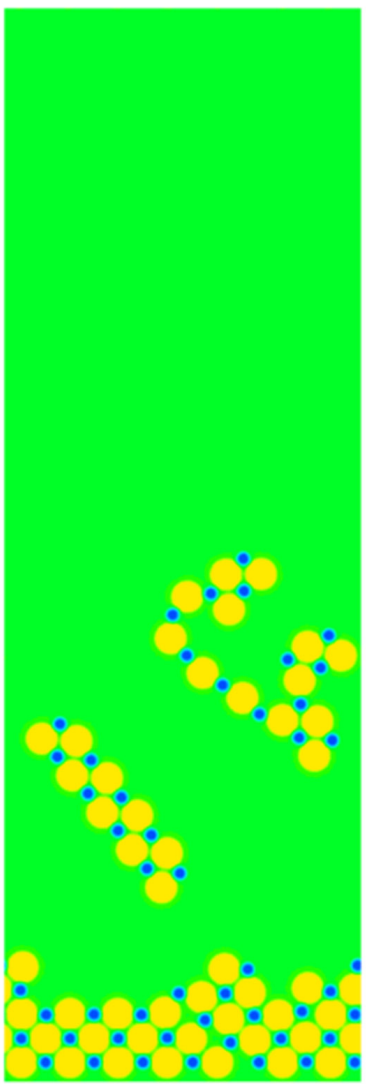

(d)
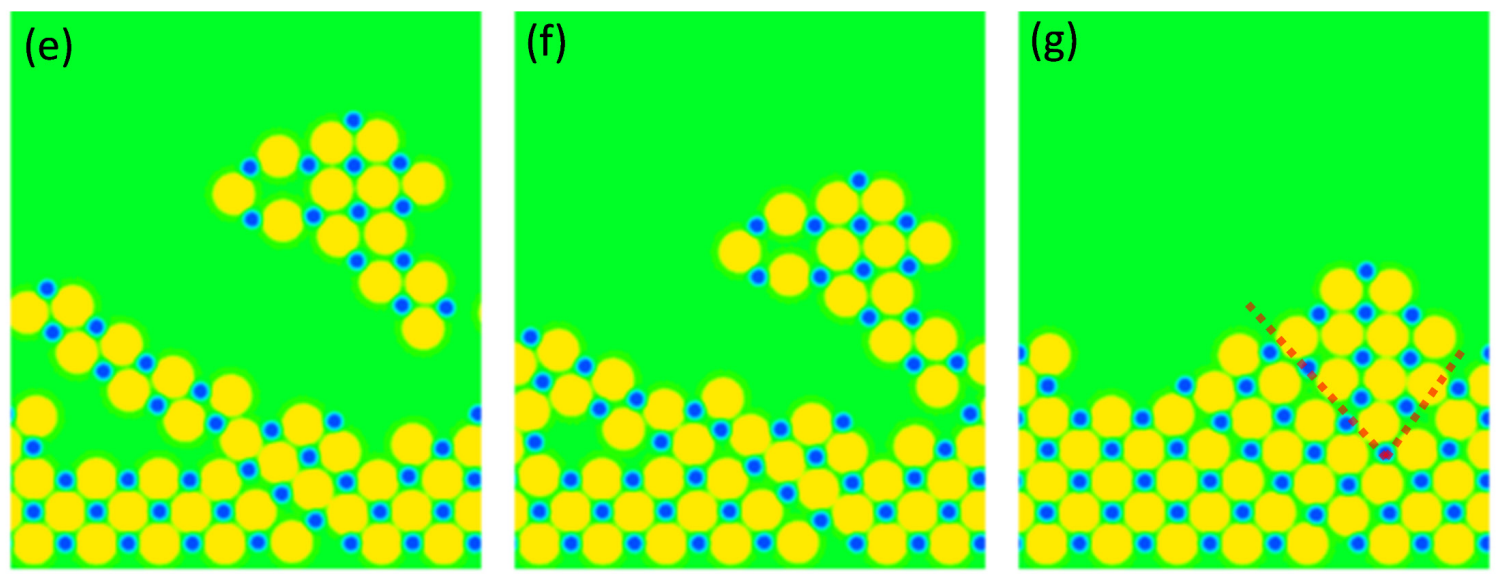

Figure 4.10 Self-assembly of colloidal particles with opposite charges under gravity. (a) Initial random distribution of particles (b)-(d) Local assembly and the clusters approach each other due to gravity (e)-(g) Final assembly process into ionic colloidal crystal (the upper vacant portion is cropped. The red dashed line in $(\mathrm{g})$ designates position of grain boundary. 
In other words, the charge amount with each particle should be limited, otherwise the thermal energy does not plays a significant role at all. In most reported experimental work, the electric double layer screens the electrostatic interaction significantly, while here we didn't consider the double layer effect thus the self-assembly process is analogous to atomic level crystallization processes.

Figure 4.10 shows the simulation results of oppositely charged particles with size ratio of $(\sqrt{2}+1): 1$. The absolute charge amount of each particle is equivalent. Initially particles are randomly dispersed (Figure 4.10a). Under gravity and electrostatic forces, particles spontaneously coagulate and move downward due to gravity (Figure 4.10b). In Figure $4.10 \mathrm{c}$, small crystallines are formed during the process of falling. In Figure $4.10 \mathrm{~d}$ small crystallines begin to merge into large crystals. Since the upper portion of the image is vacant, Figure 4.10e-f are cropped so that only the bottom parts are shown where particles are. It is interesting that one grain merges with the bottom grain perfectly without generating defects while the other does not. As a result, the final state includes obvious grain boundaries as illustrated by red dashed lines. The ionic radii perfect the requirement of the crystal symmetry, which is expected. This simulation shows the capability of DIFA model to simulate formation processes of ionic colloidal crystals, and nucleation, grain growth, and defects formation processes are demonstrated in analogy to the counterpart processes in condensed matter physics. Not shown here, if the charge amount of the particles are increased such that electrostatic energy completely dominates over $k_{B} T$ (or alternatively, the Bjerrum length is much larger than particle size), disordered heteroaggregation processes of particles are obtained. 


\subsection{Dipolar colloids self-assembly under external field control: Magnetic composites}

Magnetic particles with engineered microstructure may exhibit strong anisotropy with engineered microstructure. ${ }^{129}$ What contributes to the anisotropy is the long range magnetostatic interaction between dipolar particles. The dipole moment of each particle, however, can be either field induced, or spontaneous magnetization. Particles with field induced dipole to assembly typically requires very large external field which may limit their application. With a large spontaneous magnetization, particles only require relatively low external field to tune their self-assembly. Experimental comparison of permeability of magnetic composites before and after alignment shows obvious improvement after alignment. Our motivation is to investigate the underlying mechanism of the magnetic composites by modeling and computer simulation in order to reduce laborious experimental measurements.
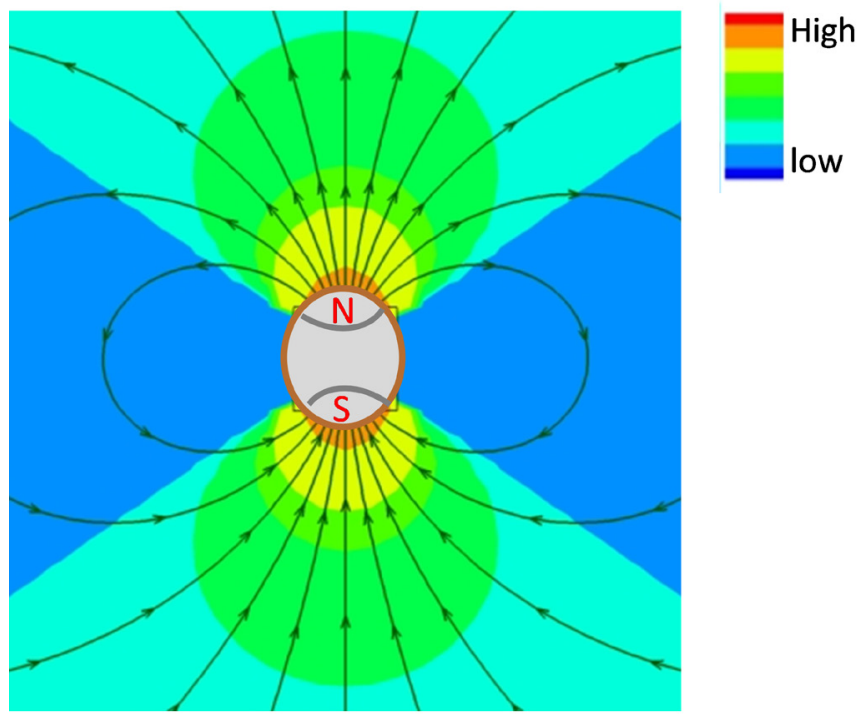

Figure 4.11 Schematic of electric/magnetic field around a dipolar particle

Modeling method has been narrated in section $2.2 \& 2.3$ (chapter 2). In this section it is assumed that each particle is distributed with unidirectional magnetization (single 
domain), unless at the surface where the magnetization smoothly transition from 1 to 0 . Figure 4.11gives a schematic of the dipole field distribution around an elliptical particle. It is clear that the electric/magnetic field has largest intensity in the vicinity of the two particle ends. The particles thus tend to spontaneously aggregate head-to-end.

Figure 4.12 shows the simulation results of self-assembly of dipolar elliptical particles under constant magnetic field. There are 85 particles totally and the external magnetic field is $H^{*}=0.3$. System size is $512 \times 512$. The elliptical particles have major semi-axes of 15 and minor semi-axes of 6 . Figure $4.12 \mathrm{a}$ is the initial configuration where particles and randomly dispersed with random orientation. Under the external field, in Figure $4.12 \mathrm{~b}$ the particles show to form short whiskers. The whiskers have a tendency to align with the external field but some are obvious not aligned or even perpendicular to the external field direction. Figure $4.12 \mathrm{c}$ shows that the whiskers keep merge to form long chains. From Figure 4.12d to $4.12 \mathrm{f}$ the long chains are straightened and aligned with the external field gradually.

In order to fully understand the composite performance dependence on structure of particle assembly, and quantitatively study the relationship between particle assembly kinetics and controlling external field, it is necessary to study the in situ property of magnetic composites during particle assembly. We use a newly developed phase field method by Wang to solve the heterogeneous distribution of magnetization in magnetic particulate composites. ${ }^{130}$ The magnetostatic free energy of the composite system is defined as ${ }^{130}$

$$
F=\int \frac{\mu_{0} M^{2}(\mathbf{r})}{2 \psi(\mathbf{r})} d^{3} r-\mu_{0} \int \mathbf{H}^{e x} \cdot \mathbf{M}(\mathbf{r}) d^{3} r+\frac{\mu_{0}}{2} \int \frac{d^{3} k}{(2 \pi)^{3}}|\mathbf{n} \cdot \tilde{\mathbf{M}}(\mathbf{k})|^{2}
$$

where $\psi(\mathbf{r})$ is the local susceptibility and $\mu_{0}$ free space permeability. The equilibrium distribution of magnetization is achieved by solving the Ginzburg-Landau type equation, given by 


$$
\frac{\partial \mathbf{M}(\mathbf{r}, t)}{\partial t}=-L \frac{\delta F}{\delta \mathbf{M}(\mathbf{r}, t)}
$$

where $L$ is a kinetic coefficient. The effective susceptibility or nominal susceptibility of the composites is defined by

$$
\bar{\chi}_{i j}=\frac{\left\langle M_{i}\right\rangle}{\left\langle H_{j}\right\rangle}
$$

The angular brackets stand for spatial average.

The evolution of susceptibility coefficients of the whole composites corresponding to the evolution process shown in Figure 4.12 is shown in Figure 4.13, where anisotropy of the final state $\left(\chi_{11}>\chi_{22}\right)$ is manifested, in contrast to the initial state of isotropic susceptibility $\left(\chi_{11} \approx \chi_{22}\right)$. It is also found that the performance of the composites begins to reach the maximum once the long chains are formed, although the long chains are not completely straightened along the external field direction.

If the particles are spherical instead of elliptical (in 2D it is circular actually), similar results are obtained. Figure 4.14 shows the simulation result self-assembly of spherical particles with magnetic dipoles under constant external field. The aligned chains appear straighter than elliptical particle case with more short whiskers coexisting. Figure 4.15 shows the internal magnetic field distribution inside the red box region in Figure 4.14. It is clear that the demagnetization is effectively eliminated at zones where particles meet (i.e., in the middle of particle chain), while in contrast inside the particles at the two ends of the long chain the internal magnetic field is greatly reduced due to demagnetization. 

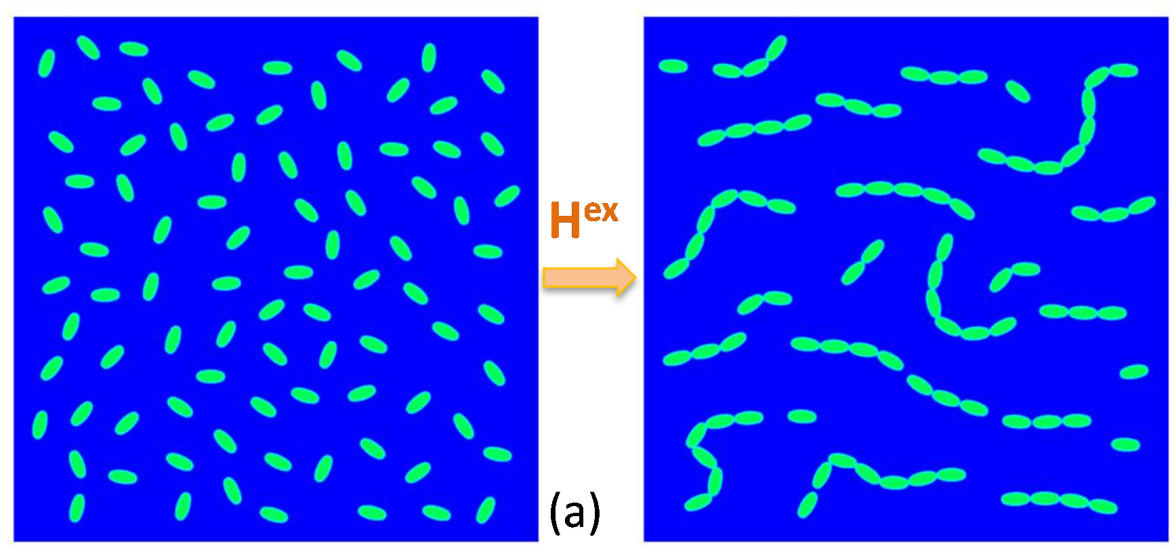

(b)
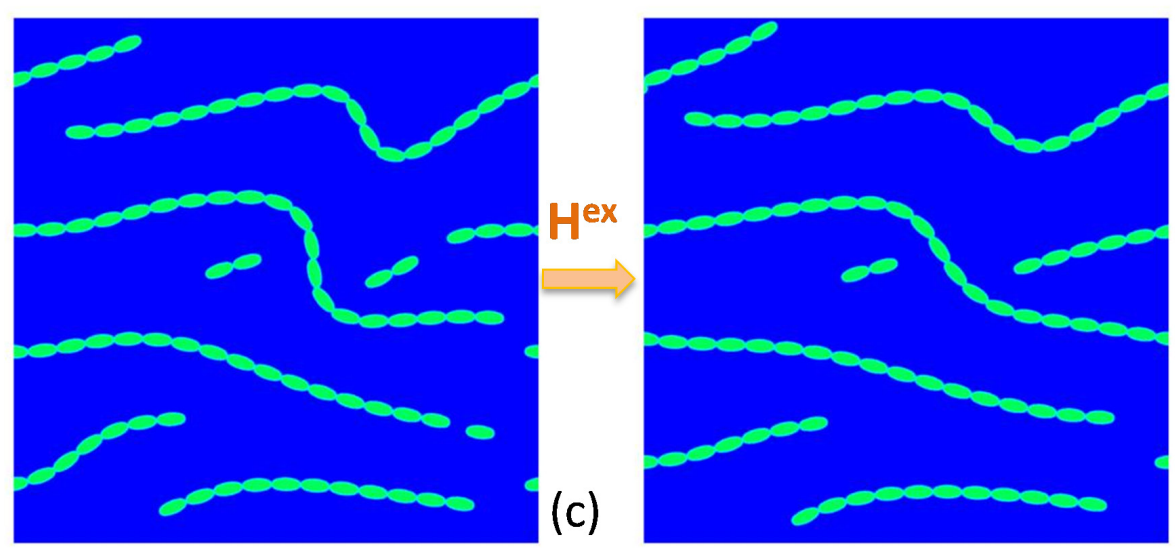

(d)
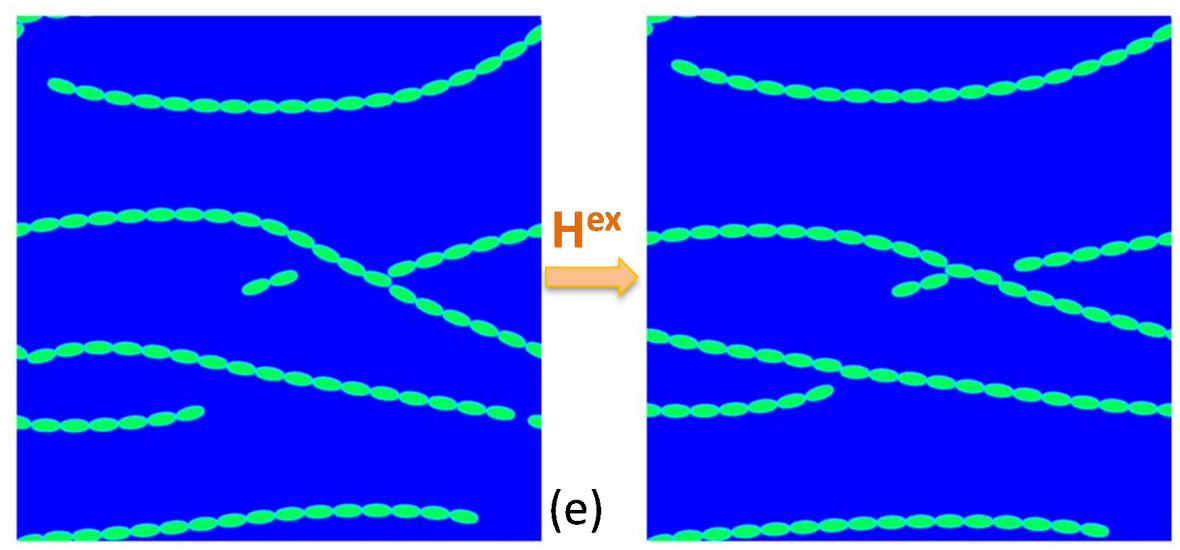

(f)

Figure 4.12 Elliptical colloidal particles with magnetic dipole alignment under constant external field $H^{e x} / M_{s}=0.3$. 


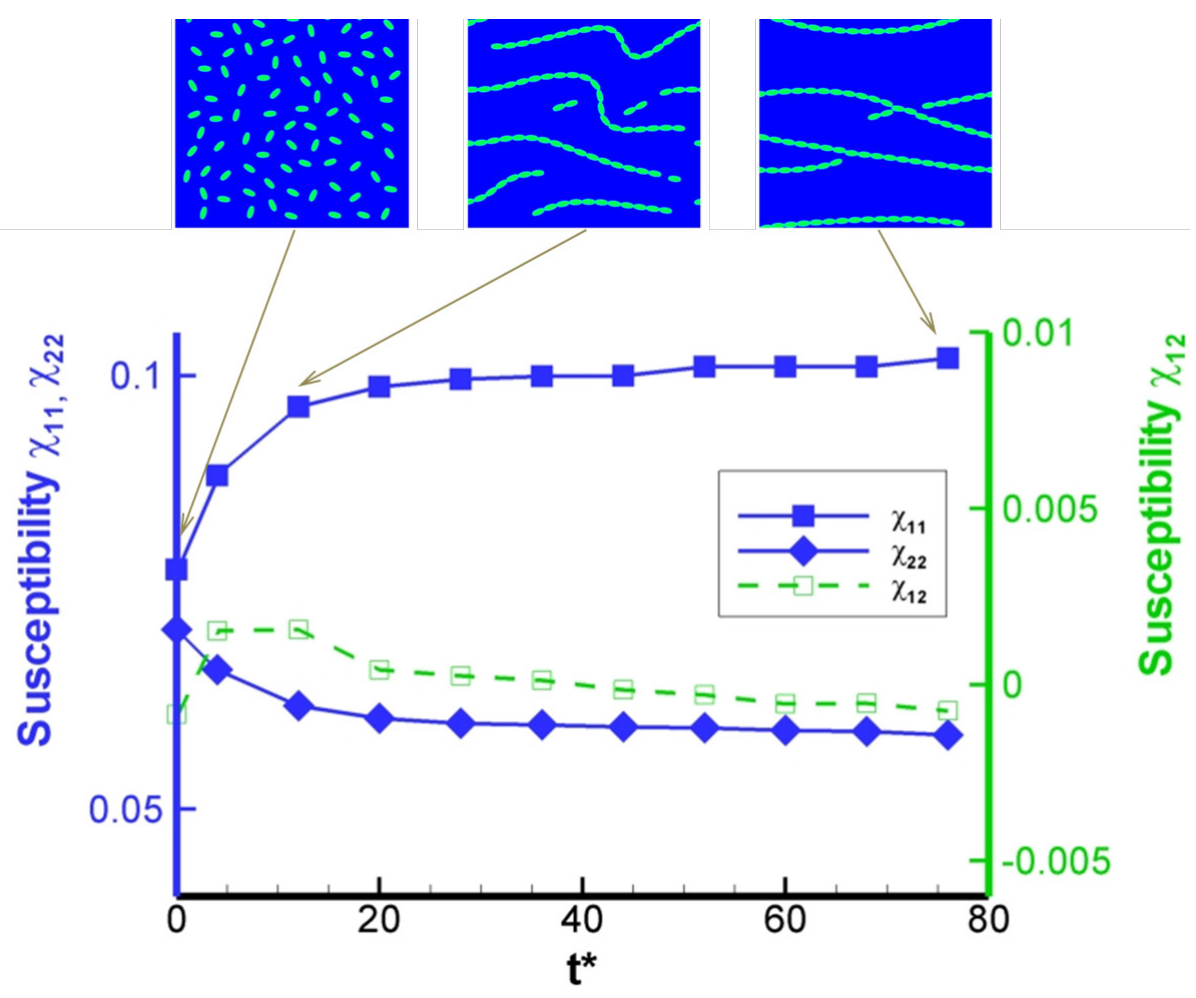

Figure 4.13 Elliptical colloidal particles with magnetic dipole alignment under constant external field, corresponding to Figure 4.12.

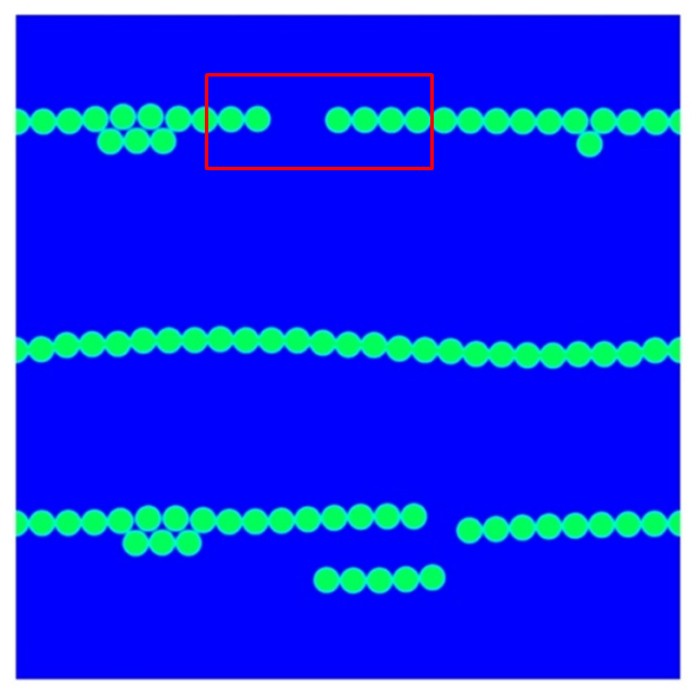

Figure 4.14 Spherical colloidal particles with magnetic dipole alignment under constant external field 


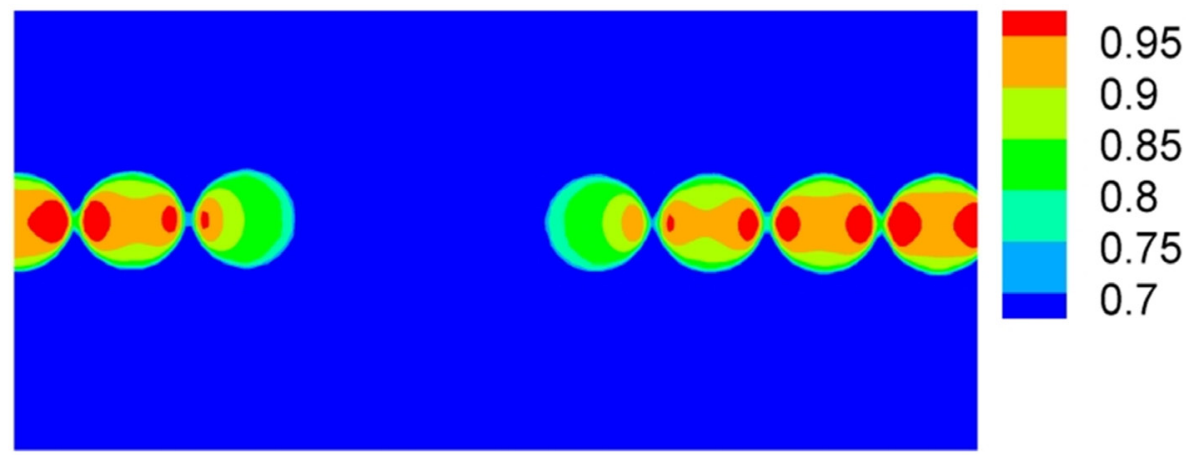

Figure 4.15 Magnetic field distribution $H / H^{e x}$ inside aligned particles (Field of view is the enclosed region of the red box in Figure 4.14.)

In the above cases the external field is applied constantly throughout the process. If the applied field is increased incrementally and gradually, there will be great difference. Figure 4.16 shows the simulation results of elliptical particles self-assembly under increasing external field. At the beginning stages, when the external field is small, the particles are reorganized and connected to each other to form rings. (Figure 4.16b-c) The rings are not broken until the external field is increased to $H^{e x} / M_{s} \approx 1.4$. In the final state all the rings are broken to form straight long chains as $H^{e x} / M_{s}$ reaches 2.0.

All the above simulation results show the anisotropy of composite susceptibility with aligned particles However, with constant external field applied from the very beginning when all particles are well dispersed distantly, only a small external field is required to align the particles. ( $H^{e x} / M_{s}=0.3$ ) With increasing external field starting from zero, however, the particles first form enclosed rings owing to dipole-dipole interaction. Once the rings are formed, strong external field are needed to break them, as is shown that the rings do not effective contribute to the anisotropy of susceptibility thus do not improve the desired performance of composites. 

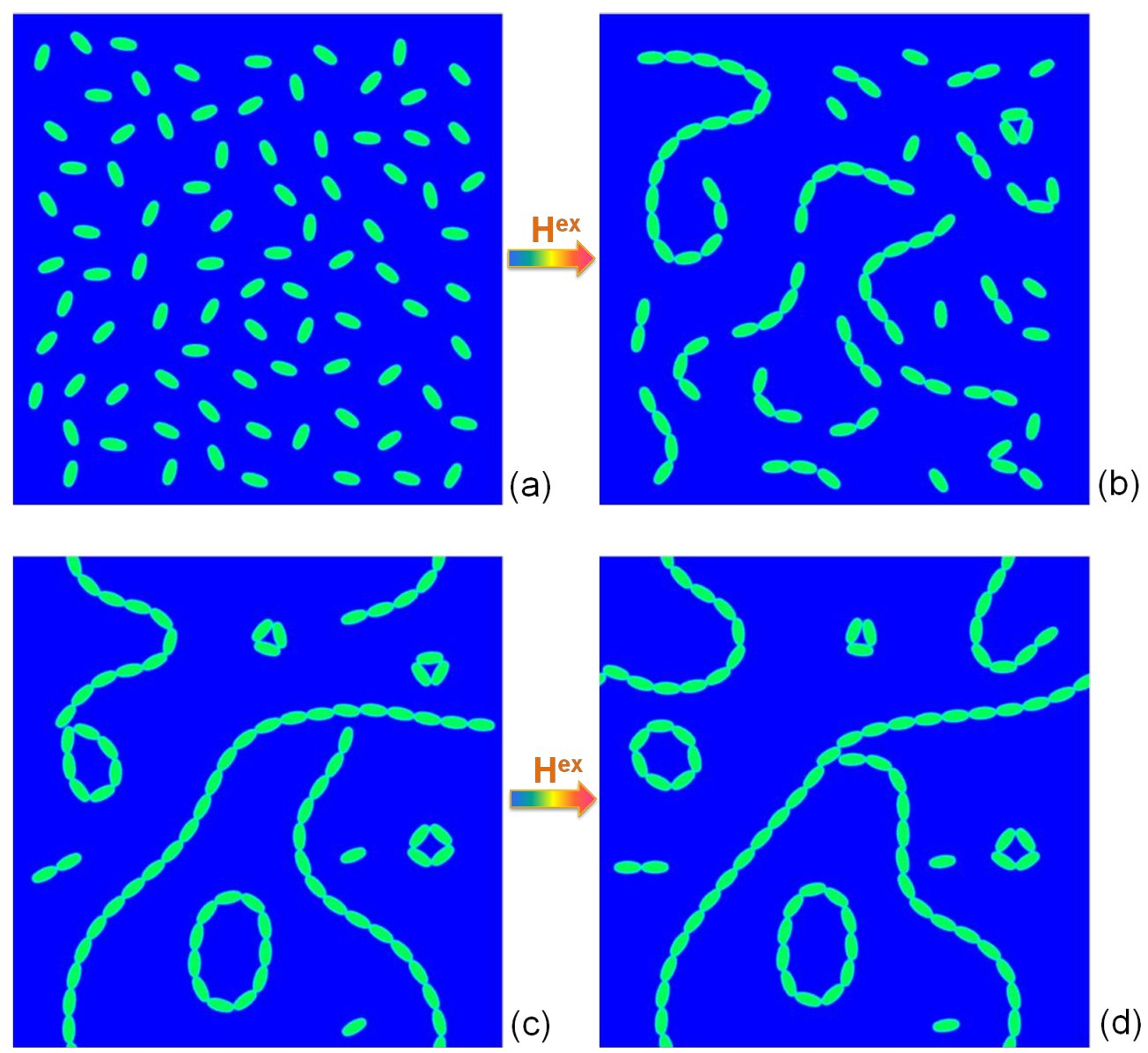

(d)
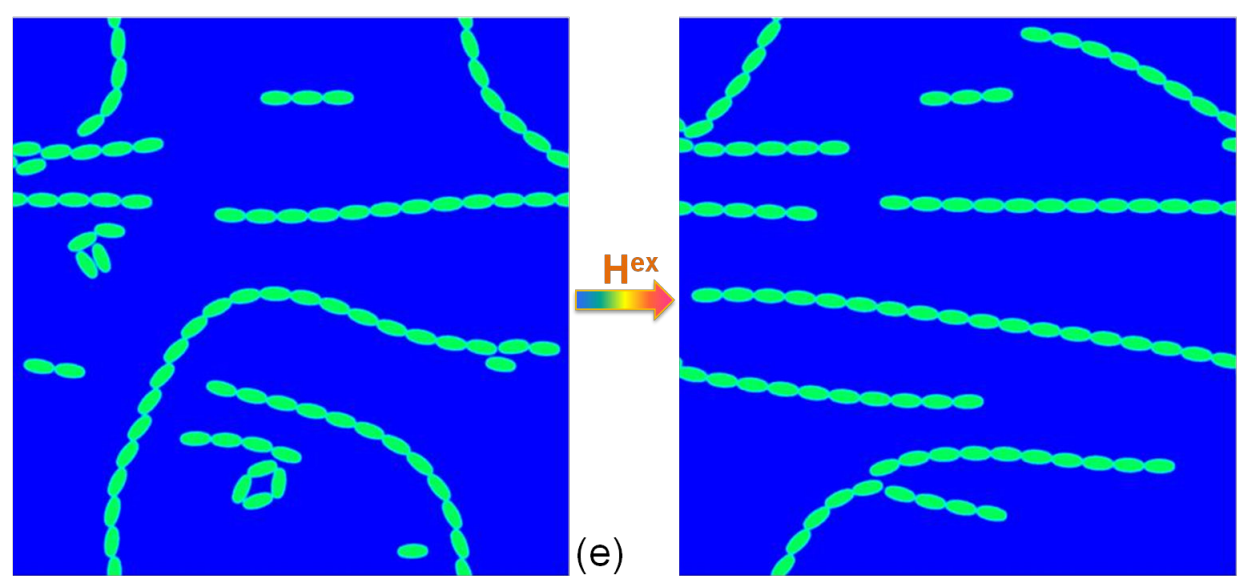

(f)

Figure 4.16 Elliptical colloidal particles with magnetic dipole alignment under increasing external field 


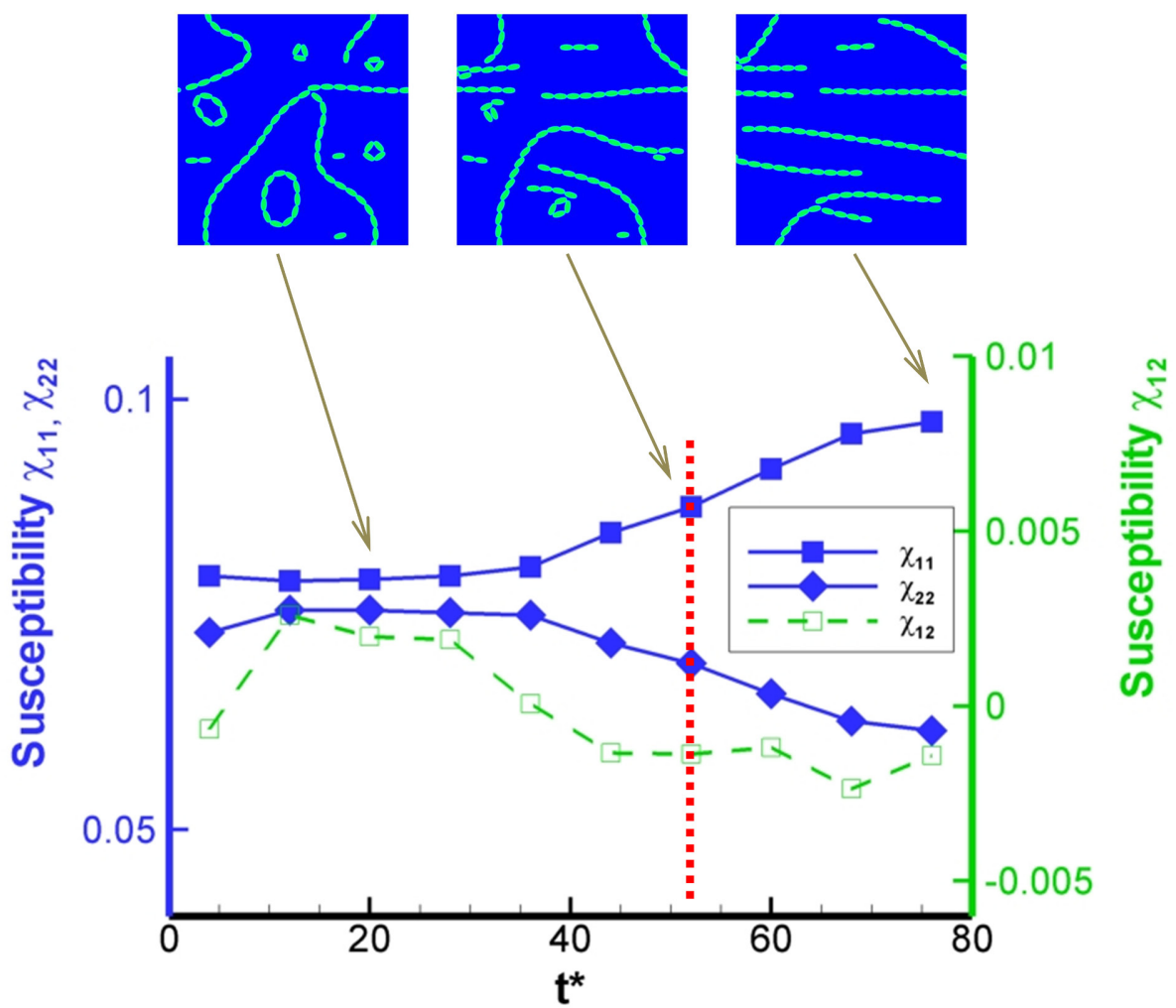

Figure 4.17 Real-time composite performance (susceptibility coefficients) evolution, corresponding to Figure 4.16. The red dotted line designates the time where rings are broken.

A quantitative comparison is given in Figure 4.18, which includes four cases, i.e., elliptical particles, spheres, coated spheres under increasing external field up to $H^{e x} / M_{s}=2.5$, except for the last case without external field. Without external field, the dipolar particles spontaneously form into rings, and as a result, the susceptibility of composites remains isotropic after particle self-assembly. For particles coated with a nonmagnetic layer of $\delta_{c} / a=0.3$, although the finally the particles are also aligned straightened lines, the susceptibility in longitudinal direction is much worse than the two cases without particle coatings. The reason is attributed to that with the presence of the nonmagnetic coating the demagnetization field impedes the magnetization of individual particles significantly. It needs to be emphasized that in all cases the particle volume fraction are the same, thus the structure of particle assembly plays the key role in 
determining the performance of composites. Our simulation results exhibit the capability of our DIFA model to simulated self-assembly of dipolar colloids with arbitrary shape, heterogeneous properties under different conditions. Our results also suggest the external field should be applied at the very beginning so as to lower the probability of formation of dipole rings, since once the rings are formed considerably high external field is required to break them.
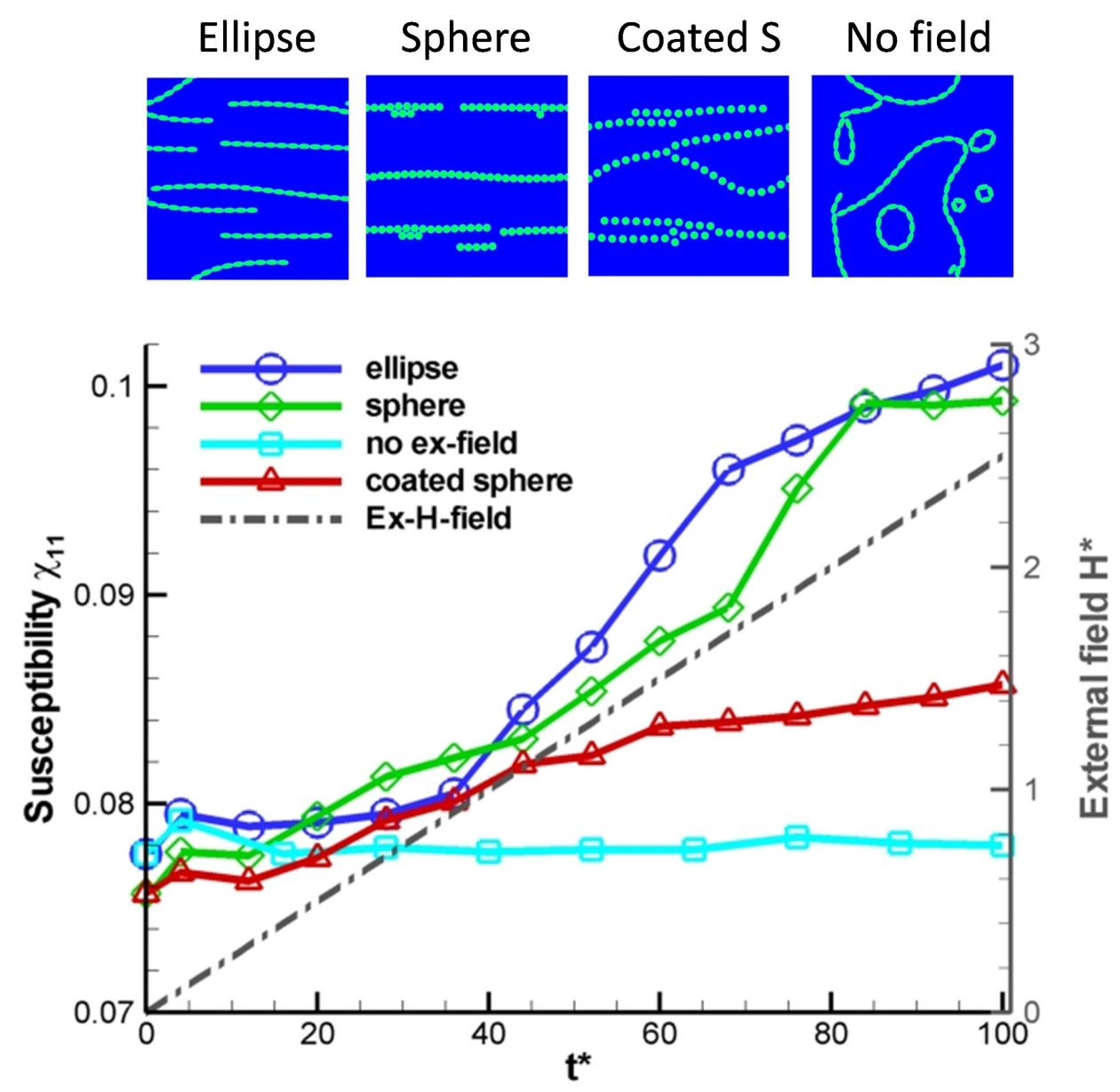

Figure 4.18 Comparison of composite performance in four cases: elliptical particles, spherical particles, coated spherical particles under increasing external field and elliptical particles self-assembly without external field applied. 


\section{Chapter 5. DIFA to modeling dipole-dipole interaction related phenomena}

General material systems are all charge-neutral. If one takes even a small amount of material (no matter metals, ceramics or polymers) out from the bulk, the positive charges can still balance negative charges. As has been discussed in Chapter 1, without net charge dipole-dipole interaction will take the most important role of electrostatic interaction. When a ferroelectric ceramic material is cooled down to below its Curie temperature, each unit cell in the crystal will be spontaneously polarized. ${ }^{131}$ The dipole-dipole interaction leads to domain phenomena and the domain evolution is a very important mechanism that determines ferroelectric response to external stimuli. ${ }^{132,133}$ For magnetic materials, since there is no real "magnetic charge", dipole-dipole interaction is the principal mechanism of magnetostatic interaction and it plays a key role in determining the magnetic domain evolution and thus macroscopic material performance. ${ }^{84}$ In this chapter, diffuse interface field modeling is built to study dipole-dipole interaction related domain behavior in ferroelectric and in ferromagnetic crystals. The model in the former is more known as Landau theory based phase field method and the in the latter known as micromagnetic modeling.

\subsection{Internal dipole field and ferroelectric domain stabilization}

\subsubsection{Phase field simulation on ferroelectric domain stabilization ${ }^{133}$ *}

The microscopic mechanism of aging and domain stabilization is attributed to shortrange ordering of charged point defects in ferroelectric materials, ${ }^{134-137}$ which provides a

* The main content of this section has been published on Applied Physics Letters (2010). 
restoring force to the domains whenever deviated from the original, aged domain configurations. In this section we perform computer modeling and simulation to investigate the aging-stabilization effects of ferroelectric domains, with a focus on the domain processes and internal bias electric field that is associated with the short-range ordering of charged point defects. For an aged ferroelectric sample with domain microstructure described by polarization field $\mathbf{P}_{0}(\mathbf{r})$, symmetry-conforming short-range ordering of charged point defects generates an effective internal bias electric field $\mathbf{E}_{0}(\mathbf{r}) .{ }^{134}$ The internal bias field is spatially heterogeneous and depends on the domain microstructure, i.e., $\mathbf{E}_{0}(\mathbf{r})$ is locally aligned with $\mathbf{P}_{0}(\mathbf{r})$ in individual domains. For convenience, we write $\mathbf{E}^{0}(\mathbf{r})=\kappa \varepsilon_{0}^{-1} \mathbf{P}^{0}(\mathbf{r})$, where $\varepsilon_{0}$ is the permittivity of free space, and $\kappa$ a parameter characterizing the internal field strength that depends on the degree of short-range ordering of charged point defects and thus the aging history. For an arbitrary domain state of such an aged ferroelectric crystal, as described by polarization field $\mathbf{P}(\mathbf{r})$, the total system free energy is formulated as described in Chapter 2, and the expansion coefficients $\alpha_{i}, \alpha_{i j}$, and $\alpha_{i j k}$ are determined from experimental data for specific material systems, e.g., $\mathrm{Pb}\left(\mathrm{Zr}_{1-x} \mathrm{Ti}_{x}\right) \mathrm{O}_{3}$ (PZT). ${ }^{138}$ Here we use $\beta_{i j k l}=\beta \delta_{i k} \delta_{j l}, L_{i j}=L \delta_{i j}$, and consider tetragonal PZT of composition $\boldsymbol{x}=\mathbf{0 . 6}{ }^{138}$

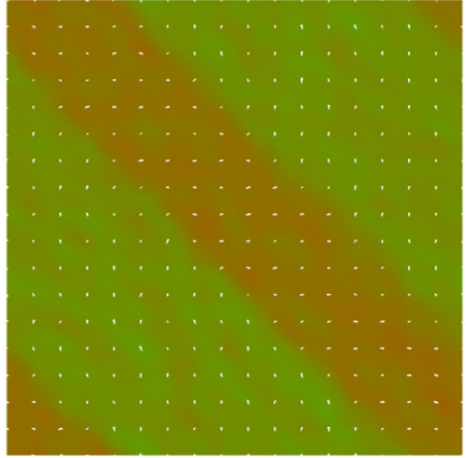

(a) $t^{*}=11$

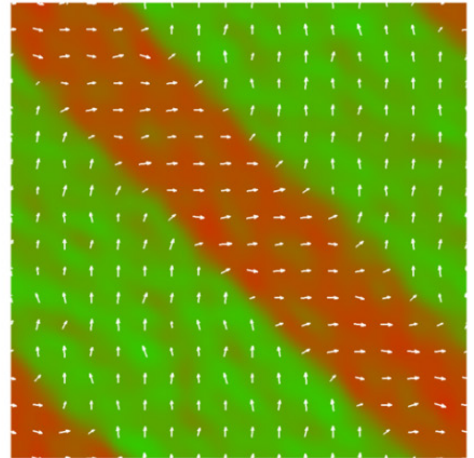

(b) $t^{*}=14$

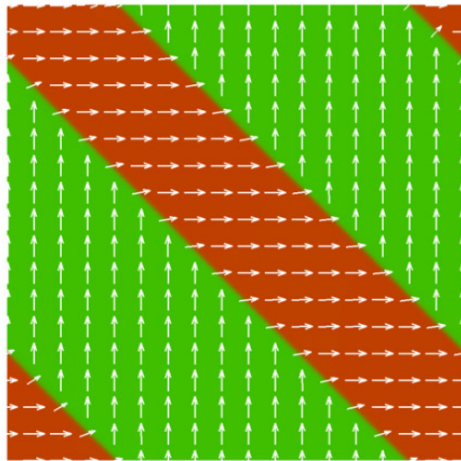

(c) $t^{*}=21$

Figure 5.1 Computer simulation of domain stabilization and recovery behaviors in sufficiently aged ferroelectrics. (a) Polarization distribution at nucleation stage of cooling-induced ferroelectric phase transition. (b) Growth of polarization vectors and 
formation of domains. (c) Fully recovered domain microstructure. Arrows represent polarization vectors. Domains are described by colors with RGB proportional to polarization components.

The computer simulation shows that domain microstructures are stabilized in sufficiently aged ferroelectric samples, where the original, aged domain configurations are automatically recovered from arbitrarily deviated states, as shown in Figure 5.1. The formula have been stated in Section 2.4.1. we write $\mathbf{E}^{0}(\mathbf{r})=\kappa \varepsilon_{0}^{-1} \mathbf{P}^{0}(\mathbf{r})$, where $\varepsilon_{0}$ is the permittivity of free space, and $\kappa$ a parameter characterizing the internal field strength that depends on the degree of short-range ordering of charged point defects and thus the aging history. The restoring force to such domain stabilization and recovery behaviors is the internal bias electric field associated. The degree of short-range ordering and, thus, strength of the internal field is characterized by the parameter, $\kappa$. In an aged sample, the short-range ordering of point defects is maintained during a diffusionless process, such as domain switching driven by a short-period external field ${ }^{134}$ or phase transformation during rapid heating-cooling cycle. ${ }^{139}$ To investigate domain stabilization and recovery phenomena, we consider a rapid heating-cooling cycle during which an aged multidomain ferroelectric sample transforms first to paraelectric phase and then back to ferroelectric phase. Since the sample stays in paraelectric state only for a short period, diffusion of point defects does not have sufficient time to alter their short-range ordering, as experimentally observed; ${ }^{139}$ upon transforming back to ferroelectric phase, the sample tends to form the same domain microstructure as in the aged state to minimize the total system free energy. An aged lamellar domain microstructure is considered in the simulations, which is the same as that shown in Figure 5.1(c) and representative of engineered domain configurations. Figure 5.1 shows the simulated domain recovery process in a sufficiently aged sample upon cooling across Curie temperature under the effect of internal bias field $1 \mathrm{kV} / \mathrm{cm}$ : Figure 5.1 (a) shows an arbitrary polarization distribution at the nucleation stage of cooling-induced ferroelectric phase transition under the effect of thermal noises; Figure 5.1(b) shows the growth of polarization vectors and 
formation of domains; and Figure 5.1(c) shows the fully recovered domain microstructure that is identical to the original, aged domain configuration. To determine the critical internal field strength required for domain recovery, a large number (over 100) of simulations are performed with different thermal noises and values of parameter $\kappa$. It is found that when internal bias field is greater than about $0.5 \mathrm{kV} / \mathrm{cm}$, domain configurations are fully recovered. In such cases, the internal field strongly affects the nucleation of ferroelectric phase upon cooling, where domains nucleate with polarization vectors aligned with local bias field directions, as shown in Figure 5.1(a). As a result, domain recovery process is very fast, as reflected in reduced simulation time.

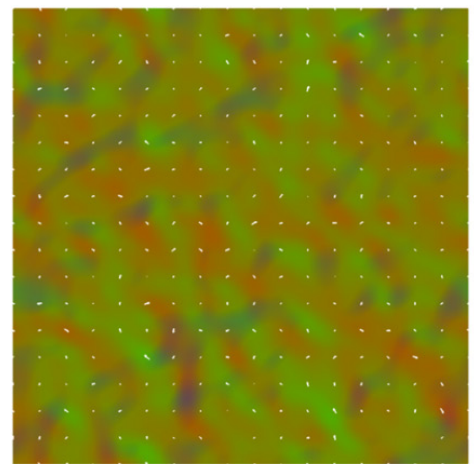

(a) $t^{*}=14$

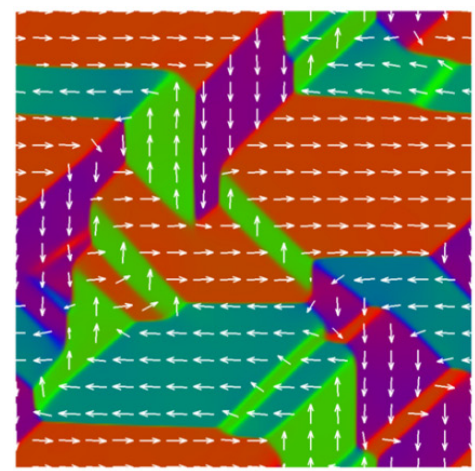

(d) $t^{*}=210$

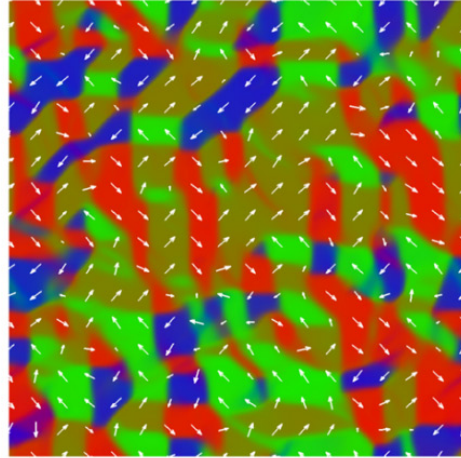

(b) $t^{*}=30$

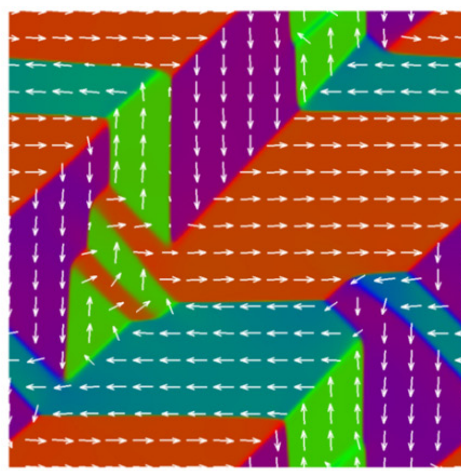

(e) $t^{*}=450$

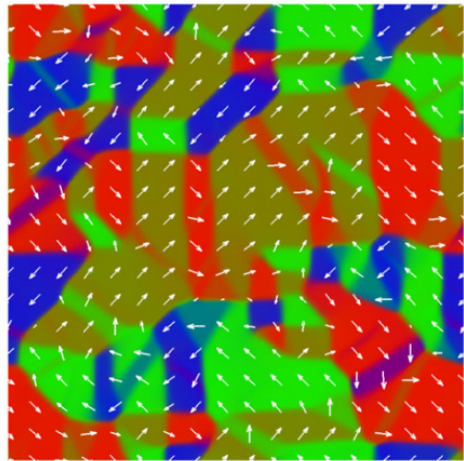

(c) $t^{*}=90$

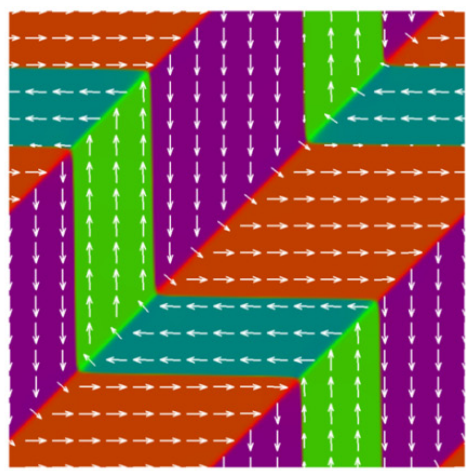

(f) $t^{*}=1200$

Figure 5.2 Computer simulation of domain evolution in insufficiently aged ferroelectrics.

The computer simulations also find that domain configurations cannot be recovered in insufficiently aged samples when internal bias field is weaker than about $0.06 \mathrm{kV} / \mathrm{cm}$. Figure 5.2 shows the simulated domain evolution process in an insufficiently aged 105 
sample upon cooling across Curie temperature under the effect of internal bias field $0.05 \mathrm{kV} / \mathrm{cm}$ : the weak internal field does not affect the nucleation of ferroelectric phase, as shown in Figure 5.2(a), and the final domain microstructure in Figure 5.2(f), which is typical of multi-domain ferroelectric states consisting of both $90^{\circ}$ and $180^{\circ}$ domain walls, is very different from the original, aged domain configuration in Figure 5.1(c). Compared to the domain evolution in sufficiently aged sample shown in Figure 5.1, domain evolution in insufficiently aged samples is slower as reflected in reduced simulation time, which is due to the more complicated domain microstructures formed along the evolution kinetic pathways, as shown in Figure 5.2(b-e). As expected, the observed domain process is similar to that in unaged samples.

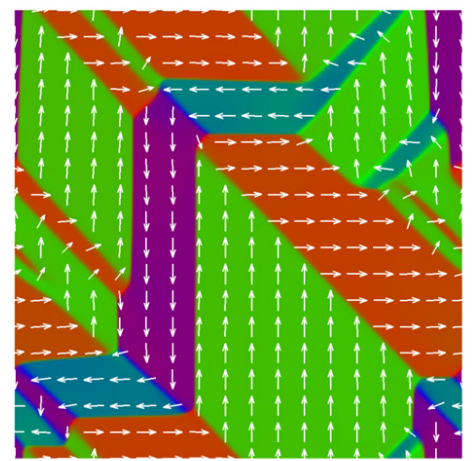

(a) $t^{*}=200$

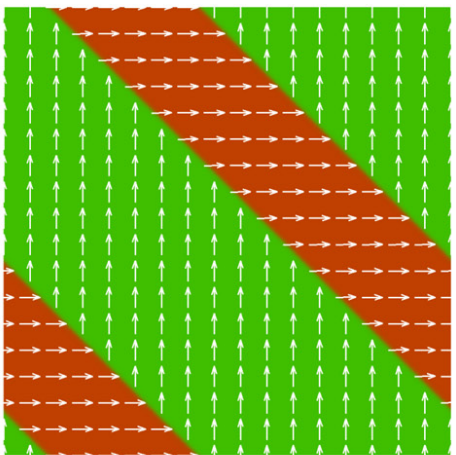

(b) $t^{*}=800$

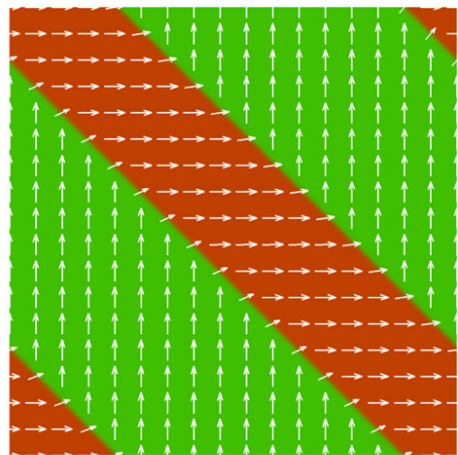

(c) $t^{*}=400000$

Figure 5.3 Computer simulation of domain evolution in intermediately aged ferroelectrics.

Figure 5.3 shows a typical example of the simulated domain evolution processes in moderately aged samples with intermediate internal bias field. The nucleation and early stage domain growth processes are similar to that shown in Figure 5.2(a-c). Nevertheless, the internal bias field, $0.07 \mathrm{kV} / \mathrm{cm}$ in this particular example, is able to eliminate some of the energetically unfavorable domains during domain evolution, leading to formation of domain microstructure similar to the original, aged domain configuration, as shown in Figure 5.3(b). However, the domain walls are displaced from the original, aged positions, as compared with Figure 5.1(c). It is found that domain configurations may still recover 
via domain wall motion but take very long relaxation time, as reflected in the reduced simulation time in Figure 5.3(c). Therefore, such very slow domain recovery process may not play a significant role in experimental observations performed during a shorter time. It is worth noting that charged point defects are usually introduced by doping, which effectively reduces domain wall mobility, as in hard PZT. Friction to domain wall motion due to point defects is not considered in the simulation, thus above intermediate internal field strength should be insufficient for complete domain recovery in real ferroelectric samples. Thus, based on the computer simulations, $0.5 \mathrm{kV} / \mathrm{cm}$ represents a lower limit for the critical internal field strength required for complete domain recovery.

To compare the simulation with experiment, we also evaluate the internal bias field strength from relevant experimental data. Based on the fact that aging-generated internal electric field produces the same effect in individual ferroelectric domains as an externally applied electric field does in electric field-induced ferroelectric phase transformation, it is expected that the Curie temperature increases with the internal field strength (i.e., degree of aging and short-range ordering of charged point defects) in aged samples. Such a phenomenon is, indeed, experimentally observed in $\mathrm{BaTiO}_{3} .{ }^{136}$ Therefore, the internal field strength can be evaluated from the Curie temperature change of aged samples. Thermodynamic analysis of electric field-induced ferroelectric phase transformation under constant pressure gives the Clausius-Clapeyron-type equation:

$$
\frac{d T}{d E}=\frac{\Delta P}{\Delta S}
$$

where $\Delta P=P_{\text {ferro }}-P_{\text {para }}=P_{\mathrm{s}}\left(T_{\mathrm{C}}\right)$ is equal to the spontaneous polarization at Curie temperature $T_{\mathrm{C}}$, and $\Delta S$ is the entropy change of ferroelectric phase transition. Using $\Delta P=0.18 \mathrm{C} / \mathrm{m}^{2}$ and $\Delta S=13.5 \mathrm{~kJ} /\left(\mathrm{m}^{3} \cdot \mathrm{K}\right)$ reported for $\mathrm{BaTiO}_{3},{ }^{131}$ we obtain $d T / d E=1.33 \times 10^{-5} \mathrm{C} \cdot \mathrm{m} \cdot \mathrm{K} / \mathrm{J}$. Experiments observe a Curie temperature change of $2 \mathrm{~K}$ in fully aged samples, ${ }^{136}$ thus the internal electric field is evaluated to be $1.5 \mathrm{kV} / \mathrm{cm}$. It is worth noting that this experimentally evaluated value in fully aged samples is higher than the critical value required for domain recovery. Therefore, simulation and experiment 
both estimate the aging-produced internal bias field in sufficiently aged ferroelectric samples on the order of magnitude of $1 \mathrm{kV} / \mathrm{cm}$.

\subsubsection{Charge Compensation and Aging Mechanisms in Iron-Doped Ferroelectric Titanate Perovskites}

During ferroelectric aging, material's properties undergo gradual changes, such as decreases in dielectric and piezoelectric constants and loss factors, increases in elastic stiffness, distortion (constriction and/or displacement) of hysteresis loop. ${ }^{131}$ It has been long recognized that such changes can be attributed to domain stabilization and memory effects developed during aging in ferroelectrics; nevertheless, clarification of the underlying mechanisms responsible for such effects requires critical examination of several possibilities, including gradual equilibration of domain structures (domain mechanism), lattice defect segregation on grain boundaries (grain boundary mechanism) or domain walls (domain wall mechanism), property change in surface layers (surface layer mechanism), and ordering of point defects inside domains (volume mechanism). ${ }^{140}$, ${ }^{141}$ While each of these mechanisms could, to a greater or lesser extent, play a role in certain systems, such as multi-domained polycrystalline ceramics, Lambeck and Jonker unambiguously showed through their elegantly designed experiments that the fundamental mechanism omnipresent in all aged ferroelectric materials is a volume effect. ${ }^{141}$ In the volume mechanism, defect dipoles inside ferroelectric domains interact with the spontaneous polarization through Lorentz-type field, gradually reorient to align with the spontaneous polarization axis inside the domains through short-range diffusion of point defects, and the short-range ordering of such charged point defects inside the ferroelectric domains manifests itself as development of an effective internal bias field to stabilize the domain structures. ${ }^{140,141}$ Such an anisotropic distribution of point defects in aged ferroelectrics is a special case of a more general phenomenon of symmetryconforming short-range ordering of point defects observed in aged low-symmetry phases of a large class of ferroic materials. ${ }^{134,139}$ 
The aging behaviors of ferroelectric materials can be controlled through manipulation of the populations of charged point defects. In addition to the naturally occurring impurities and vacancies, dopants play significant roles in enhancing or suppressing ferroelectric aging effects. ${ }^{141}$ In perovskite-type ferroelectrics, i.e., $\mathrm{ABO}_{3}$ such as barium titanate $\mathrm{BaTiO}_{3}$ and lead zirconate titanate $\mathrm{Pb}\left(\mathrm{Zr}_{1-x} \mathrm{Ti}_{x}\right) \mathrm{O}_{3}$ (PZT), due to its higher mobility, diffusion of oxygen vacancy in the oxygen octahedron has a critical role in the formation of point defect associates and their reorientation. ${ }^{141-143}$ Doping with acceptortype B-site dopants and tempering at lower oxygen partial pressure increase oxygen vacancy concentration through charge compensation mechanisms, thus enhance aging effects. ${ }^{141}$ The time, temperature and field dependences of aging behaviors can be described as a thermally activated Arrhenius process under electric field, and can be modeled by the hopping kinetics of oxygen vacancy in the oxygen octahedron surrounding a B-site acceptor dopant. ${ }^{142}$ In contrast to acceptor-type dopants, donor-type B-site dopants reduce oxygen vacancy concentration and suppress aging effects. ${ }^{141}$

When doped with aliovalent ions, charge compensation must take place. Because of their lower valency than B-site ions (e.g., $\mathrm{Ti}^{4+}$ ), acceptor-type B-site dopants (e.g., $\mathrm{Cu}^{2+}$ and $\mathrm{Fe}^{3+}$ ) carry effective negative charges with respect to the neutral lattice. These charged point defects $\left(\mathrm{Cu}_{\mathrm{Ti}}^{\prime \prime}\right.$ and $\left.\mathrm{Fe}_{\mathrm{Ti}}^{\prime}\right)$ are accompanied by the creation of charge compensating oxygen vacancies $\left(\mathrm{V}_{\mathrm{O}}^{\bullet \bullet}\right)$, which attract each other through electrostatic Coulomb force between effective charges of opposite signs to form point defect associates (Kröger-Vink defect notation ${ }^{144}$ is used, where each dot or prime represents a positive or negative charge with respect to the neutral lattice, and a cross indicates electroneutrality). For divalent dopants such as $\mathrm{Cu}^{2+}$, complete charge compensation is achieved through formation of neutral point defect associate $\left(\mathrm{Cu}_{\mathrm{Ti}}^{\prime \prime}-\mathrm{V}_{\mathrm{O}}^{\bullet \bullet}\right)^{\times}$, which also forms a defect dipole that can reorient through migration of $\mathrm{V}_{\mathrm{O}}^{\bullet \bullet}$ in the oxygen octahedron surrounding $\mathrm{Cu}_{\mathrm{Ti}}^{\prime \prime} \cdot{ }^{142,145,146}$ For trivalent dopants such as $\mathrm{Fe}^{3+}$, complete charge compensation could, in principle, be achieved through formation of neutral point defect 
associate $\left(\mathrm{Fe}_{\mathrm{Ti}}^{\prime}-\mathrm{V}_{\mathrm{O}}^{\bullet \bullet}-\mathrm{Fe}_{\mathrm{Ti}}^{\prime}\right)^{\times}$, which, however, is not observed in electron paramagnetic resonance (EPR) experiments. ${ }^{135,145}$ Although $\left(\mathrm{Fe}_{\mathrm{Ti}}^{\prime}-\mathrm{V}_{\mathrm{O}}^{\cdot \bullet}-\mathrm{Fe}_{\mathrm{Ti}}^{\prime}\right)^{\times}$is the most stable point defect associate from an energy point of view, ${ }^{135}$ it is not favored by kinetics since the low diffusion mobility of $\mathrm{Fe}_{\mathrm{Ti}}^{\prime}$ limits the probability of $\mathrm{Fe}_{\mathrm{Ti}}^{\prime}$ pair arranged in nearest neighbor $\mathrm{Ti}^{4+}$ sites. Partial charge compensation is achieved through formation of charged point defect associate $\left(\mathrm{Fe}_{\mathrm{Ti}}^{\prime}-\mathrm{V}_{\mathrm{O}}^{* \bullet}\right)^{\bullet}$, which also forms a defect dipole and can

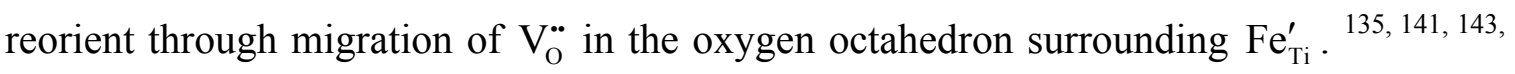
${ }^{145}$ To achieve overall charge compensation, equal amount of isolated charged point

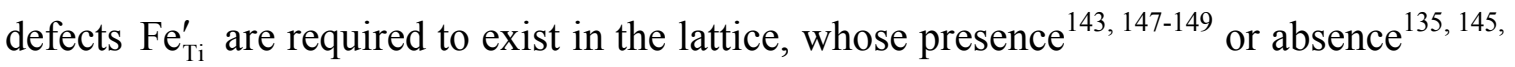
${ }^{150}$ is, however, controversially reported based on EPR experiments. Energy calculations, e.g., by density functional theory ${ }^{135,151}$ and atomistic simulation technique, ${ }^{152}$ show that isolated charged point defects $\mathrm{Fe}_{\mathrm{Ti}}^{\prime}$ have higher energy thus are not stable, as also expected from electrostatic ionic interaction between $\mathrm{Fe}_{\mathrm{Ti}}^{\prime}$ and $\mathrm{V}_{\mathrm{O}}^{*}$. Experiment shows that isolated $\mathrm{Fe}_{\mathrm{Ti}}^{\prime}$ centers are partially converted into associated $\left(\mathrm{Fe}_{\mathrm{Ti}}^{\prime}-\mathrm{V}_{\mathrm{O}}^{* \bullet}\right)^{\bullet}$ centers

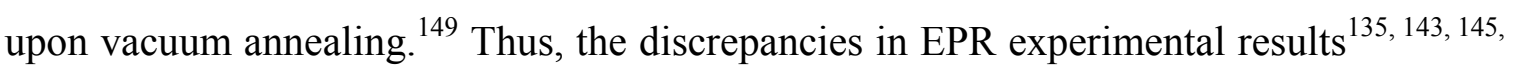
147-150, 153 could, in part, be explained by the differences in sample synthesis method and thermal treatment history. Nevertheless, it is generally observed that, even existing, the amount of isolated $\mathrm{Fe}_{\mathrm{Ti}}^{\prime}$ centers is low, thus insufficient to compensate the effective positive charges of $\left(\mathrm{Fe}_{\mathrm{Ti}}^{\prime}-\mathrm{V}_{\mathrm{O}}^{* \bullet}\right)^{\bullet}$ associates. Therefore, additional charge compensation mechanisms are required for Fe-doped titanates and similar ferroelectric perovskite systems doped with trivalent acceptor-type B-site ions. Various possibilities have been proposed and are under debate. ${ }^{135,145}$ Currently, the charge compensation mechanism remains an open question.

We hereby propose a charge compensation mechanism through formation of neutral point defect associate $\left(\mathrm{Fe}_{\mathrm{Ti}}^{\prime}-\mathrm{V}_{\mathrm{O}}^{* \bullet}-\mathrm{Ti}_{\mathrm{Ti}}^{\prime}\right)^{\times}$. We first show that the existence of $\mathrm{Ti}_{\mathrm{Ti}}^{\prime}$ and its association with $\left(\mathrm{Fe}_{\mathrm{Ti}}^{\prime}-\mathrm{V}_{\mathrm{O}}^{\bullet \bullet}\right)^{\bullet}$ are consistent with the existing experimental evidences in 
the literatures. Next, we consider dipole-dipole-like electrostatic interaction between $\left(\mathrm{Fe}_{\mathrm{Ti}}^{\prime}-\mathrm{V}_{\mathrm{O}}^{* \bullet}-\mathrm{Ti}_{\mathrm{Ti}}^{\prime}\right)^{\times}$associates and formation of defect complex involving multiple $\left(\mathrm{Fe}_{\mathrm{Ti}}^{\prime}-\mathrm{V}_{\mathrm{O}}^{\bullet \bullet}-\mathrm{Ti}_{\mathrm{Ti}}^{\prime}\right)^{\times}$associates. Finally, we show that such defect complex configurations provide memory effect and internal bias field responsible for domain stabilization and ferroelectric aging.

We consider Fe-doped titanate as a model system, such as $\mathrm{BaTiO}_{3}$ or $\mathrm{PbTiO}_{3}$. While iron could occur in variable valence states depending on sample synthesis and treatment history, such as $\mathrm{Fe}^{2+}, \mathrm{Fe}^{3+}, \mathrm{Fe}^{4+}$ and $\mathrm{Fe}^{5+}$, a majority of iron cations exist in $\mathrm{Fe}^{3+}$ valence state as acceptor-type B-site dopants, i.e., $\mathrm{Fe}_{\mathrm{Ti}}^{\prime} \cdot{ }^{135,}$ 143, 145, 147-150, 153, $154 \mathrm{Fe}_{\mathrm{Ti}}^{\prime}$ electrostatically attracts mobile $\mathrm{V}_{\mathrm{O}}^{\bullet \bullet}$ and together form associated $\left(\mathrm{Fe}_{\mathrm{Ti}}^{\prime}-\mathrm{V}_{\mathrm{O}}^{\bullet \bullet}\right)^{\bullet}$ for partial charge compensation. ${ }^{135,141,143,145}$ Because the amount of isolated $\mathrm{Fe}_{\mathrm{Ti}}^{\prime}$ centers is much lower than that of $\left(\mathrm{Fe}_{\mathrm{Ti}}^{\prime}-\mathrm{V}_{\mathrm{O}}^{* \bullet}\right)^{\bullet}$ associates, ${ }^{135,143,145,147-149,153}$ additional electrons exist in the titanates to neutralize the extra effective positive charges of $\mathrm{V}_{\mathrm{o}}^{\cdot \bullet}$. These electrons are produced during the creation of oxygen vacancies, which can be formulated as the following reaction:

$$
\mathrm{O}_{\mathrm{O}}^{\times} \rightarrow \mathrm{V}_{\mathrm{O}}^{\bullet}+2 \mathrm{e}^{\prime}+0.5 \mathrm{O}_{2}
$$

In dielectric titanates, these electrons cannot exist as free electrons; instead they are trapped on some cations, most likely on $\mathrm{Ti}^{4+}$ through the following reduction reaction: ${ }^{155}$

$$
\mathrm{Ti}_{\mathrm{Ti}}^{\times}+\mathrm{e}^{\prime} \rightarrow \mathrm{Ti}_{\mathrm{Ti}}^{\prime}
$$

Such a charge compensation mechanism is consistent with the experimental fact that $\mathrm{Ti}^{3+}$ ions (i.e., $\mathrm{Ti}_{\mathrm{Ti}}^{\prime}$ ) are widely observed in EPR experiments. ${ }^{143,}{ }^{148,}{ }^{155-157}$ This situation is similar to donor-doped titanates, and here the extra $\mathrm{V}_{\mathrm{O}}^{* \bullet}$ point defects play a role of donor dopants. It is generally accepted that "in donor-doped titanates the extra electrons are coupled with $\mathrm{Ti}^{3+} / \mathrm{Ti}^{4+}$ redox couples thus associated with $\mathrm{Ti}^{3+}$ sites $\left(\mathrm{Ti}_{\mathrm{Ti}}^{\prime}\right)$ in the perovskite structure." 157 Therefore, creation of oxygen vacancies on the regular oxygen sites (i.e., $\mathrm{V}_{\mathrm{O}}^{* *}$ ) releases neutral oxygen atoms from the lattice, and leaves behind (or 
"donates") extra electrons in the perovskite structure, which are trapped on the nearestneighbor $\mathrm{Ti}^{4+}$ cations, causing oxidation state change to $\mathrm{Ti}^{3+}$ and formation of $\mathrm{Ti}_{\mathrm{Ti}}^{\prime}$.

It is worth noting that, unlike $\mathrm{Fe}_{\mathrm{Ti}}^{\prime}$ with low diffusion mobility, the mobility of $\mathrm{Ti}_{\mathrm{Ti}}^{\prime}$ is not kinetically hindered by slow ion diffusion. Instead of ion jumping, the apparent diffusion of $\mathrm{Ti}_{\mathrm{Ti}}^{\prime}$ is through electron hopping. The electrons trapped on $\mathrm{Ti}_{\mathrm{Ti}}^{\prime}$ can be "delocalized by means of the following equilibrium:"155

$$
\mathrm{Ti}_{\mathrm{Ti}}^{\prime}+\mathrm{Ti}_{\mathrm{Ti}} \rightleftarrows \mathrm{Ti}_{\mathrm{Ti}}+\mathrm{Ti}_{\mathrm{Ti}}^{\prime}
$$

Thus, like mobile $\mathrm{V}_{\mathrm{O}}^{*}, \mathrm{Ti}_{\mathrm{Ti}}^{\prime}$ behave as mobile charged point defect as well. As a result of this behavior, these electrons contribute to the electrical conductivity of the titanates, consistent with the experimental observation of electronic conduction mechanism. ${ }^{155,156}$

Within an electrostatic picture, the charge density distribution in a perovskite lattice with point defects can be written as

$$
\rho(\mathbf{r})=\rho_{0}(\mathbf{r})+\delta \rho(\mathbf{r})
$$

where $\rho_{0}(\mathbf{r})$ is the charge density distribution of a perfect lattice without point defects (i.e., reference lattice), and $\delta \rho(\mathbf{r})$ is the effective charge density distribution associated with the point defects with respect to the reference lattice. The total electrostatic interaction energy of such a system is

$$
E=\frac{1}{8 \pi \varepsilon_{0} k} \iint_{\mathbf{r} \neq \mathbf{r}^{\prime}} \frac{\rho(\mathbf{r}) \rho\left(\mathbf{r}^{\prime}\right)}{\left|\mathbf{r}-\mathbf{r}^{\prime}\right|} d^{3} r d^{3} r^{\prime}
$$

where $\varepsilon_{0}$ is the permittivity of free space, and $k$ is a background dielectric constant. Substituting Eq. (5.5) into Eq. (5.6), the total electrostatic energy can be written as a sum of three contributions,

$$
E=E_{0}+\delta E+\Delta E
$$




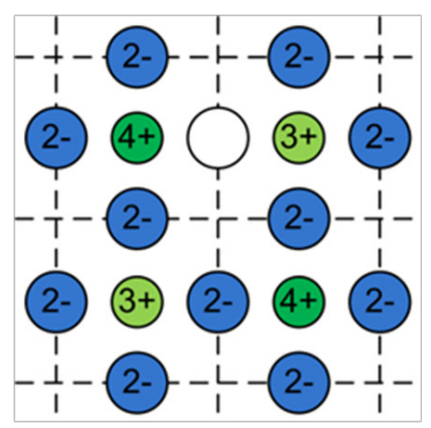

(a)

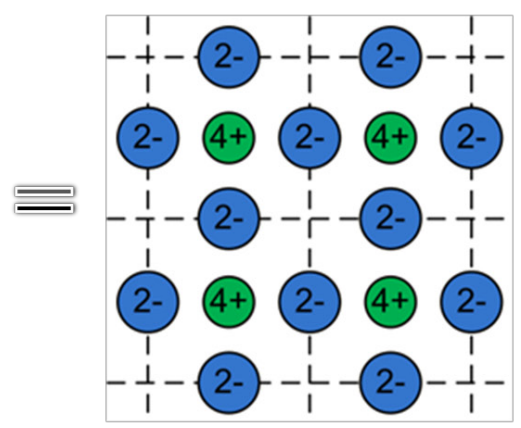

(b)

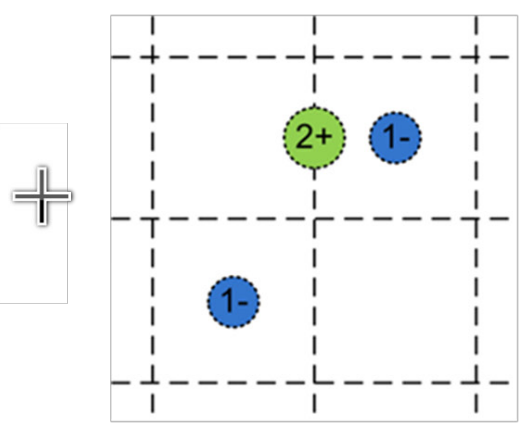

(c)

Figure 5.4 Schematic: Imperfect perovskite crystal $\mathrm{ABO}_{3}$ taken as superposition of imaginary background perfect lattice (IBL) and Effective charges (ECs). There are 2 trivalent impurity ions taking the position of $\mathrm{B}^{4+}$, and one $\mathrm{O}^{2-}$ vacancy. (Only ions in plane (200) are shown.)

The first energy contribution is

$$
E_{0}=\frac{1}{8 \pi \varepsilon_{0} k} \iint_{\mathbf{r} \neq \mathbf{r}^{\prime}} \frac{\rho_{0}(\mathbf{r}) \rho_{0}\left(\mathbf{r}^{\prime}\right)}{\left|\mathbf{r}-\mathbf{r}^{\prime}\right|} d^{3} r d^{3} r^{\prime}
$$

which corresponds to the electrostatic energy of the reference lattice, thus independent of point defect configurations. The second energy contribution is

$$
\delta E=\frac{1}{8 \pi \varepsilon_{0} k} \iint_{\mathbf{r} \neq \mathbf{r}^{\prime}} \frac{\delta \rho(\mathbf{r}) \delta \rho\left(\mathbf{r}^{\prime}\right)}{\left|\mathbf{r}-\mathbf{r}^{\prime}\right|} d^{3} r d^{3} r^{\prime}
$$

which corresponds to the electrostatic interaction energy of the charged point defects and depends on point defect configurations. The third energy contribution is

$$
\Delta E=\frac{1}{4 \pi \varepsilon_{0} k} \iint_{\mathbf{r} \neq \mathbf{r}^{\prime}} \frac{\delta \rho(\mathbf{r}) \rho_{0}\left(\mathbf{r}^{\prime}\right)}{\left|\mathbf{r}-\mathbf{r}^{\prime}\right|} d^{3} r d^{3} r^{\prime}
$$

which describes the interactions between the effectively charged point defects and the reference lattice. Eq. (5.10) can be rewritten as

$$
\Delta E=\int \delta \rho(\mathbf{r}) \varphi_{0}(\mathbf{r}) d^{3} r
$$

where 


$$
\varphi_{0}(\mathbf{r})=\frac{1}{4 \pi \varepsilon_{0} k} \int_{\mathbf{r} \neq \mathbf{r}^{\prime}} \frac{\rho_{0}\left(\mathbf{r}^{\prime}\right)}{\left|\mathbf{r}-\mathbf{r}^{\prime}\right|} d^{3} r^{\prime}
$$

which describes the electrostatic potential field inside the reference lattice. Within a fully ionic picture as often adopted in the defect chemistry of oxides, the effective charge density distribution of the point defects can be written as a sum over all defect ions,

$$
\delta \rho(\mathbf{r})=\sum_{\mathbf{r}_{\mathrm{O}}} 2 e \delta\left(\mathbf{r}-\mathbf{r}_{\mathrm{O}}\right)-\sum_{\mathbf{r}_{\mathrm{B}}} e \delta\left(\mathbf{r}-\mathbf{r}_{\mathrm{B}}\right)
$$

where $e$ is the elementary charge, and $\mathbf{r}_{\mathrm{O}}$ and $\mathbf{r}_{\mathrm{B}}$ describe the positions of $\mathrm{V}_{\mathrm{O}}^{\bullet \bullet}$ and $\mathrm{B}$-site defects ( $\mathrm{Fe}_{\mathrm{Ti}}^{\prime}$ and $\left.\mathrm{Ti}_{\mathrm{Ti}}^{\prime}\right)$, respectively. Inserting Eq. (5.13) into Eq. (5.11) yields

$$
\Delta E=\sum_{\mathbf{r}_{\mathrm{O}}} 2 e \varphi_{0}\left(\mathbf{r}-\mathbf{r}_{\mathrm{O}}\right)-\sum_{\mathbf{r}_{\mathrm{B}}} e \varphi_{0}\left(\mathbf{r}-\mathbf{r}_{\mathrm{B}}\right)
$$

Because the electrostatic potential inside the reference lattice is identical at all equivalent lattice sites, i.e., all O-sites or all B-sites, the electrostatic energy in Eq. (5.14) only depends on the numbers of individual types of point defects in the lattice, but does not depend on the point defect configurations. Therefore, the point defect configurations are determined only by the energy term $\delta E$ in Eq. (5.9).

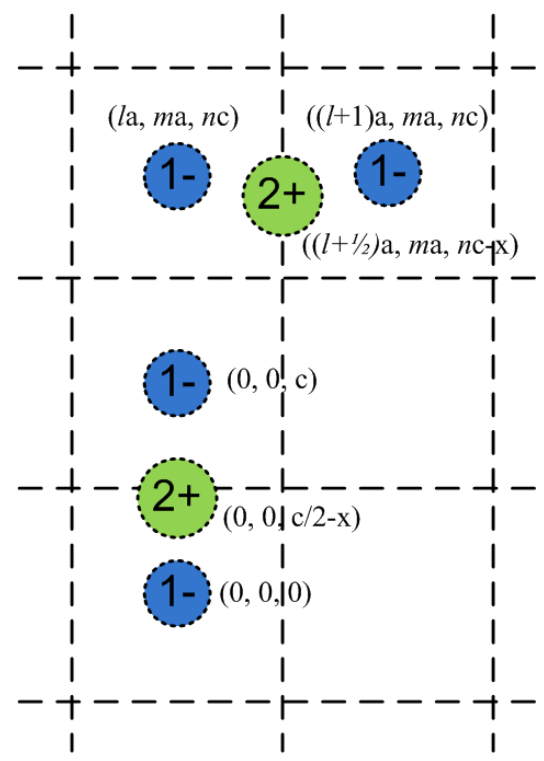

Figure 5.5 (a) Perpendicular two shoulder dipoles

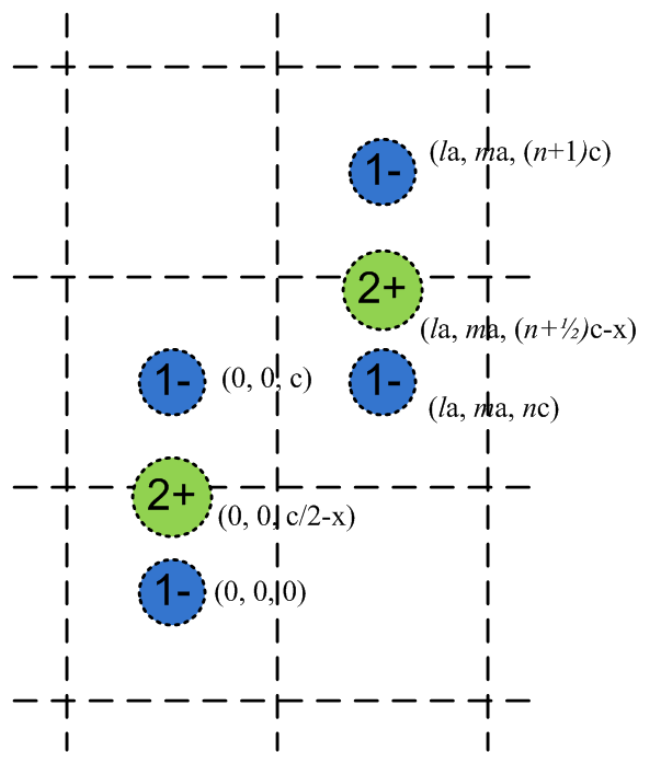

(b) Parallel two shoulder dipoles 
Within the picture of the effective charges, calculation of the electrostatic energy between the effective charges suggests that the shoulder type $\left(F e_{T i}{ }^{\prime}-V_{O}{ }^{\prime \bullet}-T i_{T i}{ }^{\prime}\right)^{\times}$is neutral and stable. However, under this configuration the bias electric field would be zero due to symmetry. If this neutral dipole takes effect in aging, the symmetry must be broken. If one considers dipole-dipole interaction, there is difference. Assume there are two perpendicular shoulder-like dipoles as shown in Figure 5.5a, and two parallel shoulder-like dipoles as shown in Figure 5.5b. Energy minimized configuration of two dipoles interaction among all possible configurations is two close perpendicular dipoles, with $l=0, m=0, n=2$ (as appeared in Figure 5.5a) or equivalent configurations by symmetry. Electrostatic energy difference of this "L" type two should-like dipole complex between two opposite polarization direction is $\Delta \varphi^{*}=-0.973 d / c_{t}$ (where $d$ is the effective displacement of $T i$ ion upon ferroelectric phase transition), which for $\mathrm{BaTiO}_{3}$ gives $\Delta \varphi=-0.12 \mathrm{eV}$. It shows that "L" type dipole associate prefers the polarization direction to the bottom of the letter "L" (upward in Figure 5.5a).

The effective bias internal electric field $\mathbf{E}_{\text {bias }}$ caused by the "L" type two dipole complex is given by

$$
\Delta \varphi \cdot X / 2=\left[-\mathbf{E}_{\text {bias }} \cdot \mathbf{P}-\left(-\mathbf{E}_{\text {bias }} \cdot(-\mathbf{P})\right)\right] \cdot V_{\text {unit cell }}
$$

where $\Delta \varphi=\varphi(d)-\varphi(-d)$ is the energy difference between two opposite polarization directions, and $X$ is the fraction of the doping defects. Take $|\mathbf{P}| \approx P_{s}$ (spontaneous polarization), one obtains $E_{\text {bias }}=0.6 \mathrm{kV} / \mathrm{cm}$, which is in agreement with the estimated required strength from both phase-field simulation and the Clausius-Clapron type equation for field induced ferroelectric phase transformation, $0.5 \sim 1 \mathrm{kV} / \mathrm{cm} .{ }^{133}$

It is worth noting that such ionic model is based upon the assumption that the lattice does not relax around point defects, which, of course, is an approximation. Nevertheless, 
as shown by first-principles total energy method, ${ }^{158}$ the lattice relaxation for neutral point defect complexes is much smaller in magnitude than for isolated charged point defects. Thus, such an ionic model is reasonable for neutral point defect associate

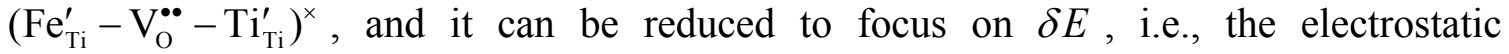
interactions only among the effectively charged point defects.

\subsection{Magnetic dipole-dipole interaction and magnetization processes in FePt polytwin crystals under external field ${ }^{84}$ *}

\subsubsection{Introduction}

The ordered $\mathrm{L} 1_{0}$ phase of FePt exhibits one of the highest magnetic anisotropy energy and is a promising candidate for high density magnetic recording media and high energy product materials in micro-electromechanical systems (MEMS). ${ }^{159-161}$ The $\mathrm{L} 1_{0}$ phase has a tetragonal lattice with its magnetic easy direction along the tetragonal c-axis. Since the ordered $\mathrm{L} 1_{0}$ phase forms from the cubic disordered A1 phase, the ordering reaction produces three crystal variants with tetragonal axes along $<100>$ directions, and each variant forms two antiphase domains. The lattice misfit between different crystal variants raises elastic energy of the FePt system during ordering process, which can be effectively reduced by a formation of stress-accommodating crystallographic microstructures. ${ }^{162}$ The typical microstructures observed in bulk FePt are multilayers composed of two alternating $\{110\}$ twin-related crystallographic variants, so-called polytwin structures, which are free of misfit stress. FePt polytwin crystals have been extensively investigated to understand the relationship between microstructures and magnetic properties. In particular, the effects of two major crystal defects in FePt polytwin crystals, namely $\{110\}$ twin boundaries and antiphase domain boundaries, on magnetic domain structures and

\footnotetext{
* The main content of this section has been published on Journal of Applied Physics (2010).
} 
magnetic properties have been studied experimentally and theoretically. ${ }^{163-169}$ In this section, we investigate in detail the effects of twin boundaries on magnetization processes by using micromagnetic modeling and computer simulations.

The magnetization process in a ferromagnet is operated through magnetic domain rotation or its wall motion or both. While typical magnetic domain configurations in both demagnetized and magnetized FePt polytwin crystals have been systematically studied, ${ }^{163-165,168,169}$ detailed magnetic domain processes between these initial and final states are not clear and need further investigations. In FePt polytwin crystals, the magnetization processes have been analyzed by magnetic domain rotation model under various magnetic field directions. ${ }^{168}$ However, the predicted remanent magnetization and coercivity from the magnetization rotation model are significantly different from the experimentally measured values. It is worth noting that, due to the very high magnetocrystalline anisotropy of $\mathrm{FePt}$, magnetic domain rotation is significant only at very high magnetic field. Therefore, instead of magnetic domain rotation, it is domain wall motion that dominates the magnetization processes in FePt polytwin crystals, as observed in experiments. ${ }^{163,165}$ In this work we employ micromagnetic modeling and perform computer simulation to investigate the magnetic domain behaviors in $\mathrm{FePt}$ polytwin crystals under applied magnetic fields along different crystallographic directions. The simulation results show the detailed magnetic domain structure evolutions through domain wall motion, reveal the important roles of the magnetostatic interaction and the competition between the internal and external magnetic fields, explain the magnetic responses to external magnetic fields in different directions, and analyze the effects of twin boundaries on the magnetization processes in FePt polytwin crystals.

\subsubsection{Micromagnetic modeling details}

In the model, polytwin crystal structure of $\mathrm{L}_{0}$ phase is described by spatially distributed vector field $\mathbf{t}(\mathbf{r})$ that characterizes the local orientation of tetragonal axis, and magnetic domain structure by magnetization vector field $\mathbf{M}(\mathbf{r})$. The tetragonal axis $\mathbf{t}(\mathbf{r})$ 
changes from one variant to another across twin boundaries and is constant over the time, corresponding to a given polytwin FePt crystal. Magnetization vector $\mathbf{M}(\mathbf{r})$ has a constant magnitude $M_{\mathrm{s}}$ (saturation magnetization) but its direction changes from one domain to another and evolves with time under external field, whose average value over the whole sample determines the magnetic response of the material. Given the constant magnetization vector length, the unit directional vector field $\mathbf{m}(\mathbf{r})=\mathbf{M}(\mathbf{r}) / M_{\mathrm{s}}$ is conveniently used to describe the magnetic domain structure. The total system free energy is a sum of magnetocrystalline energy, exchange energy, magnetostatic energy, and external magnetic energy. ${ }^{169}$ (see Section 2.4 )

For numerical calculations, it is convenient to express the equations of the free energy and magnetization evolution in their reduced forms: ${ }^{169}$

$$
\begin{aligned}
F^{*}= & \frac{F}{\mu_{0} M_{s}^{2} l^{3}}=\int K_{1}^{*}\left\{1-[\mathbf{t}(\mathbf{r}) \cdot \mathbf{m}(\mathbf{r})]^{2}\right\} d^{3} r^{*}+A^{*} \int|\operatorname{grad} \mathbf{m}(\mathbf{r})|^{2} d^{3} r^{*} \\
& +\frac{1}{2} f \frac{d^{3} k^{*}}{(2 \pi)^{3}}\left|\mathbf{n} \cdot \tilde{\mathbf{m}}\left(\mathbf{k}^{*}\right)\right|^{2}-\int \mathbf{h}^{\mathrm{ext}} \cdot \mathbf{m}(\mathbf{r}) d^{3} r^{*}
\end{aligned}
$$

and

$$
\frac{\partial \mathbf{m}\left(\mathbf{r}^{*}, t^{*}\right)}{\partial t^{*}}=-\mathbf{m} \times \frac{\delta F^{*}}{\delta \mathbf{m}}+\alpha \mathbf{m} \times\left(\mathbf{m} \times \frac{\delta F^{*}}{\delta \mathbf{m}}\right)
$$

where the reduced spatial coordinates are obtained by normalization with respect to the length unit of the computational grid size $l$ as $r_{i}^{*}=r_{i} / l$, the reduced reciprocal coordinates $k_{i}^{*}=k_{i} l$, the reduced time $t^{*}=\left(1+\alpha^{2}\right) t / \gamma M_{s}$, and the dimensionless parameters $K_{1}^{*}=K_{1} / \mu_{0} M_{s}^{2}$ and $A^{*}=A / \mu_{0} M_{s}^{2} l^{2}$.

Using FePt material parameters $M_{s}=1.14 \times 10^{6} \mathrm{~A} / \mathrm{m}, K_{1}=6.6 \times 10^{6} \mathrm{~J} / \mathrm{m}^{3}$, and $A=10^{-}$ ${ }^{11} \mathrm{~J} / \mathrm{m},{ }^{8}$ the typical magnetostatic interaction energy is $K_{d}=\mu_{0} M_{s}^{2} / 2=8 \times 10^{5} \mathrm{~J} / \mathrm{m}^{3}$, characteristic lengths $\delta_{W}=\pi \sqrt{A / K_{1}}=3.9 \mathrm{~nm}$ and $\delta_{e x}=\sqrt{A / K_{d}}=3.5 \mathrm{~nm}$, and $K_{1}^{*}=4$. In the simulations, we choose $A^{*}=3.1$ that gives the computational grid size $l=1.4 \mathrm{~nm}$, the 
damping parameter $\alpha=10$, and the reduced time increment $\Delta t^{*}=0.01$. A $512 \times 512$ computational cell with periodic boundary condition is used to describe the magnetic domain evolution in a representative volume of FePt polytwin crystals. The simulations are performed on Lonestar supercomputer at Texas Advanced Computing Center using our own Fortran 90 code with parallel algorithm by Message Passing Interface (MPI) and fast Fourier transform (FFT) technique, which has been developed in our group and used in previous works. ${ }^{85,86}$

The simulations start with the magnetic domain configuration in a representative polytwin structure composed of two twin-related crystallographic variants, as shown in Figure 5.6. In the figure, magnetic domain structure is visualized with both the magnetization vectors (black color arrows) at every 30 grid points and the color contour plot of the magnetization vector component in the twin boundary normal direction. As such, the same color across a twin boundary indicates the magnetic continuity and thus no magnetic charge at the twin boundary. The initial domain structure in Figure 5.6 is a demagnetized energy-minimizing configuration where magnetization vectors are aligned with their respective underlying tetragonal axes, forming $180^{\circ}$ domains within individual twin variants and $90^{\circ}$ domains across twin boundaries. ${ }^{168,170}$ As shown in Figure 5.6, there are four magnetic domain variants, namely $\mathrm{V} 1, \mathrm{~V}^{\prime}, \mathrm{V} 2$, and $\mathrm{V} 2$ ' in the two twin variants. We consider five cases, namely A-E, where the external magnetic fields are applied along different crystallographic directions. The simulated magnetization curves and the corresponding magnetic domain structures are shown in Figure 5.7. 

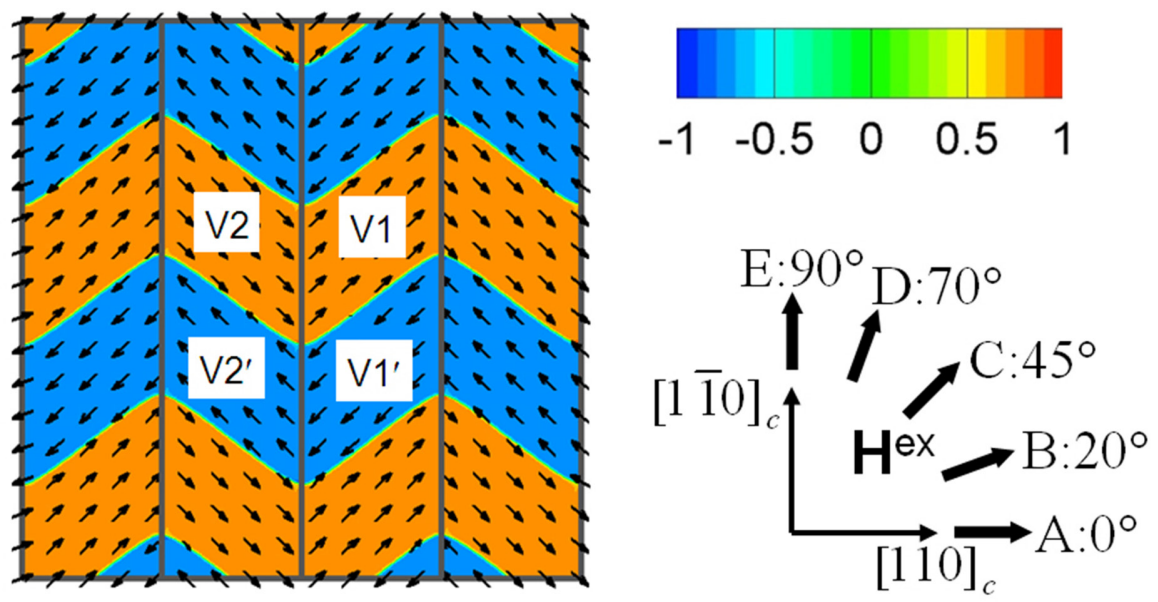

Figure 5.6 Initial magnetic domain configuration, crystallographic orientation, and applied field directions. The straight vertical lines are twin boundaries (also $90^{\circ}$ domain walls) and the tilted lines separating two different color regions are $180^{\circ}$ domain walls. The magnetic domain structure is described by both the arrows (at every 30 grid points) representing the magnetization vector directions and the color contour representing magnetization vector component in the twin normal direction $[110]_{c}$. The magnetization vectors align with the tetragonal c-axes of respective twin variants and form the energy minimizing and demagnetized domain configuration composed of four magnetic domain variants, namely $\mathrm{V} 1, \mathrm{~V}^{\prime}$, $\mathrm{V} 2$, and $\mathrm{V}^{\prime}$. External magnetic fields are to be applied along the five different directions as illustrated.

\subsubsection{Simulation results and discussion}

Figure 5.7 shows that the magnetization processes under magnetic fields along different orientations are mainly operated through $180^{\circ}$ magnetic domain wall motions. The originally continuous $180^{\circ}$ domain wall segments in the two twin variants move either in the same or the opposite directions, depending on the external magnetic field directions. $180^{\circ}$ domain walls in both twin variants eventually collide and annihilate under increasing magnetic field, producing single-domain variants. This results in two 
types of the final magnetic domain states, i.e., head-to-tail (A2, B2 and C3) and head-tohead (D3 and E2) configurations, which is consistent with previous studies. ${ }^{163,168}$

It is shown that, once these final magnetic domain states are reached, the magnetization curves flatten and increase slowly with increasing field. Since there are no more $180^{\circ}$ magnetic domain walls to move, these later stage magnetization curves correspond to solely the magnetic domain rotation, which is difficult due to the high magnetic anisotropy. Such later stage magnetization processes under high magnetic fields along different directions can be quantitatively studied by magnetic domain rotation model. ${ }^{168}$ In the following, we focus on the early stage magnetization curves and analyze the magnetic domain evolutions by $180^{\circ}$ domain wall motions. In particular, the different characteristics of the magnetization curves under magnetic fields along various directions are explained based on the simulation results shown in Figure 5.7.

For case A where magnetic field is applied along the normal direction of the twin planes, V1 and V2 are energetically favored over $\mathrm{V}^{\prime}$ and $\mathrm{V} 2^{\prime}$ by the external field. It is seen that $180^{\circ}$ domain wall segments $\mathrm{V} 1 / \mathrm{V}^{\prime}$ and $\mathrm{V} 2 / \mathrm{V} 2^{\prime}$ move together, growing $\mathrm{V} 1$ and $\mathrm{V} 2$ at the expense of $\mathrm{V}^{\prime}$ and $\mathrm{V}^{\prime}$ as shown in $\mathrm{A} 1$. The $180^{\circ}$ domain walls move until they collide and annihilate to form a single domain state in each twin variant as shown in A2, where magnetization vectors align closely along the easy magnetic directions of respective twin variants and form head-to-tail configuration. A rapid increase of the magnetization curve $\mathrm{A}$ is due to the $180^{\circ}$ domain wall motions shown in $\mathrm{A} 1$, while its flattened part is due to the magnetic domain rotation of A2 configuration. 


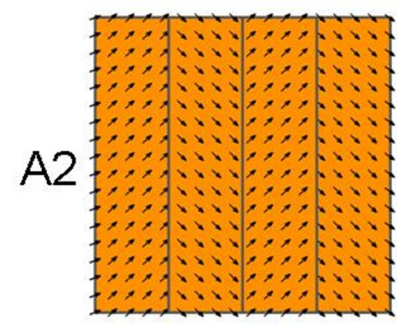

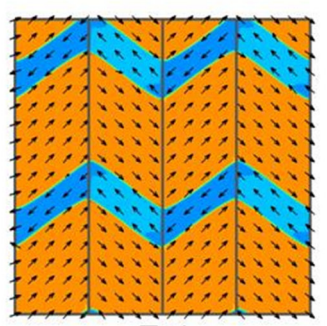

B1

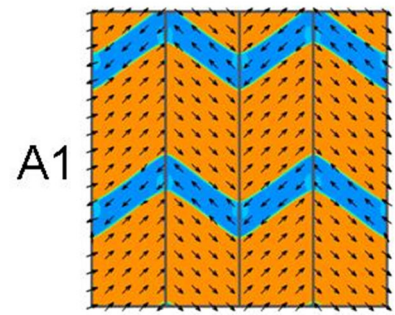

E2

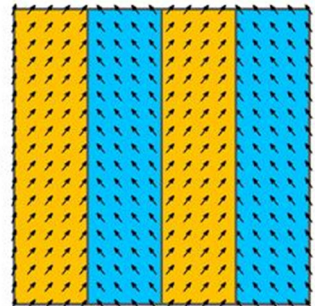

E1

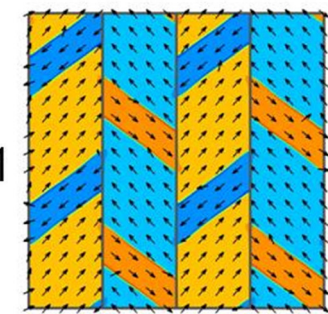

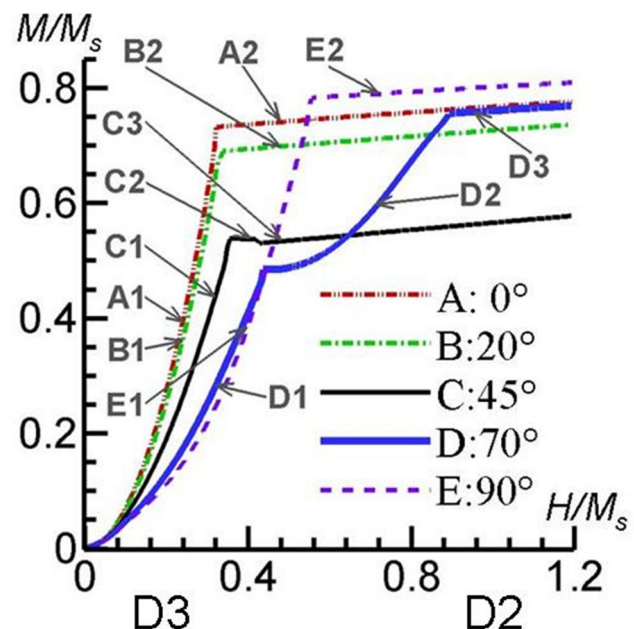

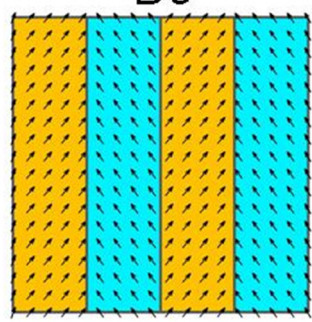

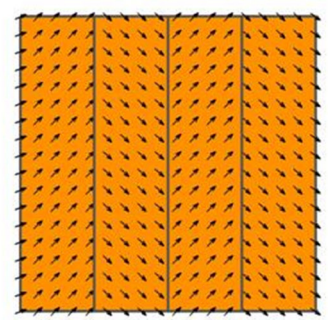

B2
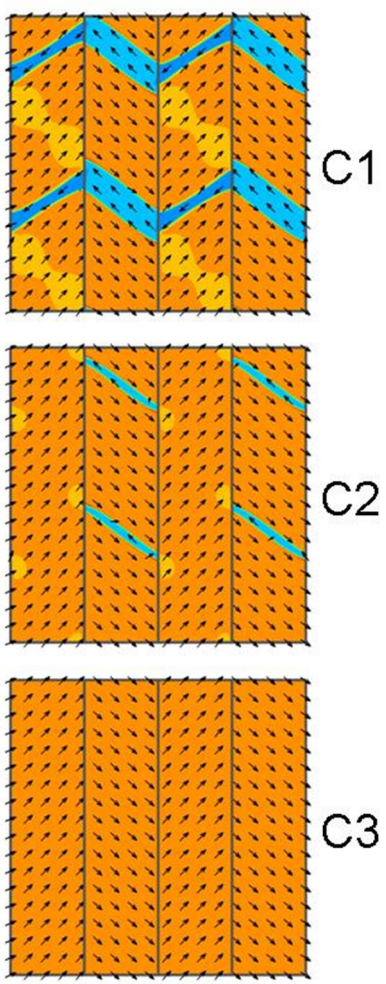

C3

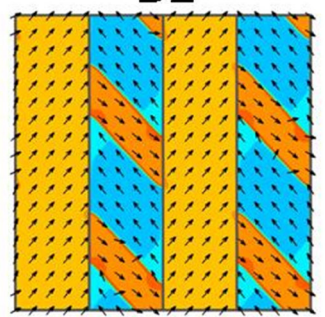

Figure 5.7 Simulated magnetization curves and corresponding magnetic domain structures during magnetization processes under external magnetic fields along the five different directions shown in Figure 5.6. Black arrows represent magnetization vector distribution, whose component in the twin normal direction is represented by color contours according to the color scale in Figure 5.6. Close-up views of the highlighted rectangular regions in $\mathrm{C} 1$ and $\mathrm{C} 2$ are shown in Figures 5.9 and 5.10, respectively.

Since the $180^{\circ}$ domain walls maintain the continuity across the twin boundaries during their motions, it provides magnetic continuity and thus does not generate magnetic charges at the twin boundaries. In other words, magnetostatic interaction does not 
produce internal magnetic field. A similar magnetization process is observed for case B. Comparison of $\mathrm{B} 1$ and $\mathrm{A} 1$, however, reveals different characteristics in their magnetic domain structures: the $180^{\circ}$ domain wall segments $\mathrm{V} 1 / \mathrm{V}^{\prime}$ and $\mathrm{V} 2 / \mathrm{V} 2^{\prime}$ are symmetric with respect to twin boundary in A during their motions while they are not symmetric anymore in case B. In particular, the shape of $\mathrm{V} 1 / \mathrm{V}^{\prime}$ ' walls is concave while that of $\mathrm{V} 2 / \mathrm{V}^{\prime}$ walls is convex in the domain structure of B1, as highlighted in Figure 5.8, where the domain walls clearly develop different curvatures in different twin variants. It is because the driving forces by external magnetic field to the $180^{\circ}$ domain wall segments $\mathrm{V} 1 / \mathrm{V}^{\prime}$ and $\mathrm{V} 2 / \mathrm{V}^{\prime}$ are different, thus $\mathrm{V} 1 / \mathrm{V}^{\prime}$ moves faster than $\mathrm{V} 2 / \mathrm{V} 2^{\prime}$, promoting their splitting at twin boundaries. The reason that they are still connected is to avoid the magnetic charge generation associated with the $180^{\circ}$ domain wall splitting. ${ }^{85}$ As a result, the joints at twin boundaries pull back the fast-moving $\mathrm{V} 1 / \mathrm{V}^{\prime}$ ' walls while pull forward the slow-moving V2/V2' walls, which results in different curvatures and bending of the $180^{\circ}$ domain walls in different twin variants near the twin boundaries. Comparison of the magnetization curves A and B shows that, in spite of the same final configurations A2 and B2, magnetization curve B is lower as compared with $\mathrm{A}$. It is simply due to the different projections of the magnetization vectors to the applied field directions in cases $\mathrm{A}$ and $\mathrm{B}$, which also explains a further decreased magnetization curve $\mathrm{C}$.
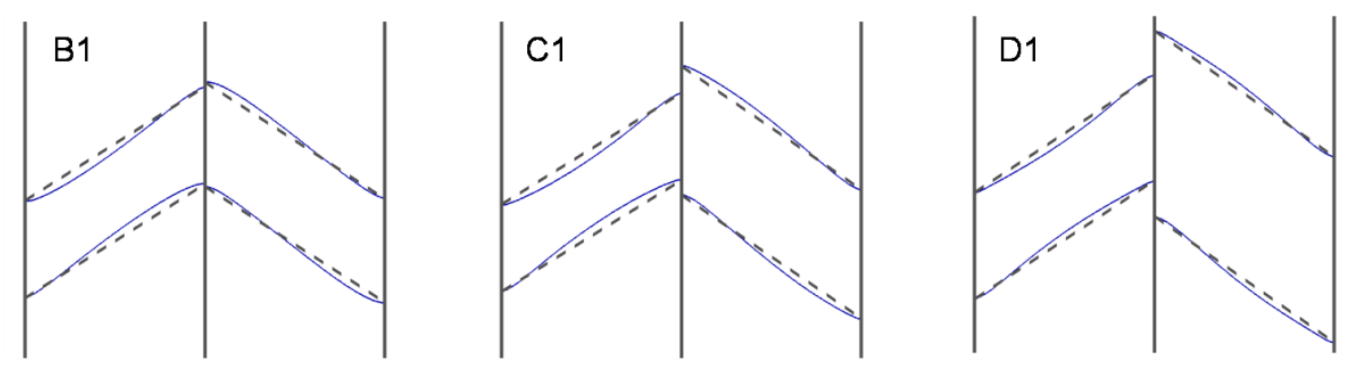

Figure 5.8 Bending of magnetic domain wall segments in the configurations B1, C1, and D1, respectively, shown in Figure 5.7. Blue solid curved lines delineate domain walls. Grey dashed straight lines are plotted as guide to eye to highlight the different domain wall curvatures developed in the two twin variants: bowing inward in variant 1 (concave shape) while bowing out in variant 2 (convex shape). 
In case $\mathrm{C}$, external magnetic field is applied perpendicular to the easy direction of the twin variant 2. In addition to the different curvatures of $\mathrm{V} 1 / \mathrm{V}^{\prime}$ and $\mathrm{V} 2 / \mathrm{V}^{\prime}$ walls as observed in $\mathrm{B} 1$, a new phenomenon of $180^{\circ}$ domain wall splitting is observed in $\mathrm{C} 1$, as highlighted in Figure 5.8. Due to the splitting, magnetic charges are generated at the twin boundaries, generating internal magnetic field, as shown in Figure 5.10 by color contour and vector plot respectively. As the splitting generates internal magnetic field and increases magnetostatic interaction energy, higher external magnetic field is required to move domain walls as compared with cases $\mathrm{A}$ and $\mathrm{B}$, which explains the lowered magnetization curve in its early stage. The heterogeneous magnetization rotation as observed by the lighter color patch in $\mathrm{C} 1$ is due to the internal magnetic field. $\mathrm{C} 1$ also shows that, in spite of splitting, V2/V2' walls move in the same direction as the previously connected $\mathrm{V} 1 / \mathrm{V}^{\prime}$ walls, and their lagging behind leads to an intermediate stage of single-domain $\mathrm{V} 1$ state and multi-domain $\mathrm{V} 2 / \mathrm{V}^{\prime}$ state in the two twin variants as observed in $\mathrm{C} 2$. Such a domain configuration composed of single-domain and multidomain twin variants under external magnetic field along one twin variant's easy direction has been experimentally observed in a multilayer structure. ${ }^{171}$ As in $\mathrm{C} 1$, heterogeneous magnetization rotation caused by the internal magnetic field is observed in $\mathrm{C} 2$ as reflected in the lighter color patches (see details in the close-up view in Figure 5.10). It is the collision of $\mathrm{V} 1 / \mathrm{V}^{\prime}$ walls in the twin variant 1 that flattens the magnetization curve since the motions of $\mathrm{V} 2 / \mathrm{V} 2$ ' walls solely in $\mathrm{C} 2$ configuration do not contribute to the sample magnetization along the applied field. It is interesting to notice a small downward step (drop) in the magnetization curve corresponding to the collision of $\mathrm{V} 2 / \mathrm{V}^{\prime}$ walls that changes multi-domain state of the twin variant 2 in $\mathrm{C} 2$ to a singledomain state in the final $\mathrm{C} 3$ configuration. This small magnetization drop appearing in the transition from $\mathrm{C} 2$ to $\mathrm{C} 3$ is associated with the annihilation of $180^{\circ}\left(\mathrm{V} 2 / \mathrm{V}^{\prime}\right)$ domain walls whose existence contributes a small magnetization component to the total magnetization along the external magnetic field direction. 


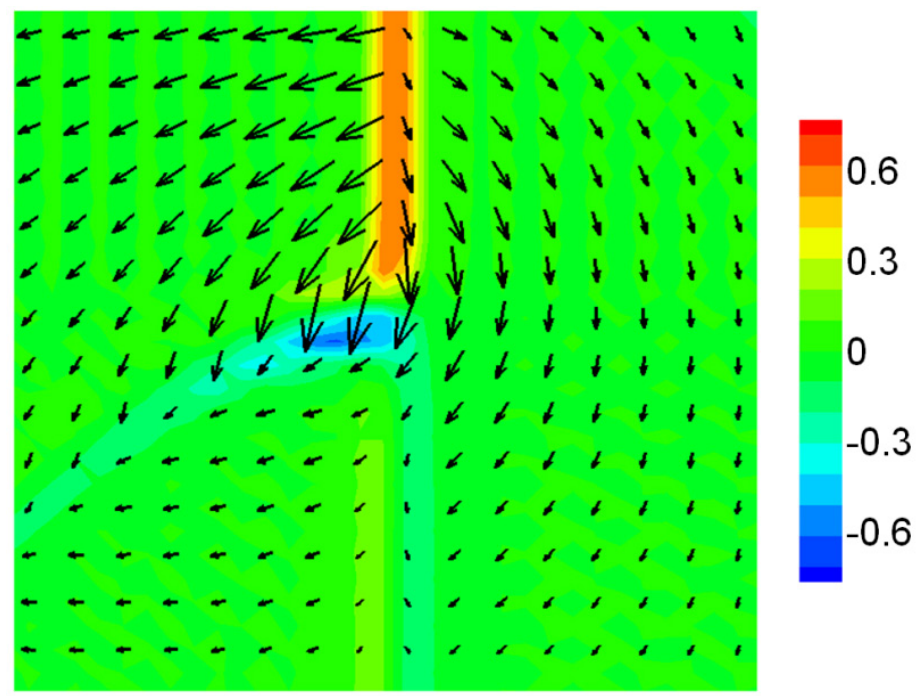

Figure 5.9 Close-up view of magnetic charge density and internal magnetic field distributions in the highlighted rectangular region of the domain configuration $\mathrm{C} 1$ in Figure 5.7 The color contour plot represents the magnetic charge density distribution, and the vector plot describes the internal magnetic field.

As shown in Figure 5.10, the magnetic domain walls in variant 2 and the heterogeneous internal magnetic field lead to heterogeneous magnetization distribution in C2. This heterogeneous magnetization distribution in $\mathrm{C} 2$ contributes a small magnetization along the applied magnetic field. In particular, as shown in Figure 5.10, magnetization vectors at $\mathrm{V} 2 / \mathrm{V}^{\prime}$ walls align along the external field direction that is perpendicular to the walls. Such heterogeneity no longer exists in C3 by forming a uniform domain in variant 2 where magnetization vectors align along the easy direction perpendicular to the external field, providing zero contribution to the magnetization along applied field direction and, thus, resulting in the small drop in the magnetization curve $\mathrm{C}$. The motion of $\mathrm{V} 2 / \mathrm{V}^{\prime}$ walls in variant 2 is to decrease internal magnetic field by reducing magnetic charge due to magnetic discontinuity. Since in the twin variant 2 , the magnetization vectors align close to its easy direction that is perpendicular to the applied field, the final state $\mathrm{C} 3$ offers the lowest average magnetization over the two twin variants as compared to other cases shown in Figure 5.7. 
When external magnetic field is applied further away from the twin plane normal in case D, it is found that the final domain state is different from cases A-C. Unlike the magnetic charge free twin boundaries in A2, B2 and C3, the twin boundaries in the final configuration D3 are magnetically charged. The resulting internal magnetic field causes additional magnetic domain rotation in both twin variants, contributing to the higher magnetization under high field than in case B. Since the projections of the easy magnetization directions of the twin variants to the external field directions of cases $B$ and $\mathrm{D}$ are the same, without the extra rotation due to the internal magnetic field in case D the magnetization curves would give the same value for the two final configurations. It is shown in D1 and highlighted in Figure 5.8 that the originally connected V1/V1' and $\mathrm{V} 2 / \mathrm{V}^{\prime}$ walls split and move in the opposite directions due to different driving force directions by the external field, and show different curvatures as in case C. The magnetization curve shows clearly distinctive three stages, corresponding to three typical domain structures shown in D1, D2, and D3. The change from D1 to D2 occurs as V1/V1' walls collide and annihilate, and D3 appears when V2/V2' walls collide and annihilate. In the magnetization curve $\mathrm{D}$, while the third stage corresponds to magnetic domain rotation, the first and second stages are due to magnetic domain wall motions. The reduction in $180^{\circ}$ domain walls that are magnetization carriers upon the annihilation of $\mathrm{V} 1 / \mathrm{V} 1^{\prime}$ walls explain the significantly reduced slope of the second stage with respect to the first stage.

Case E considers an external magnetic field parallel to the twin planes. As in case D, $\mathrm{V} 1 / \mathrm{V}^{\prime}$ and $\mathrm{V} 2 / \mathrm{V}^{\prime}$ walls move in the opposite directions leading to the final domain state that is magnetically charged, as shown in E1 and E2. Different from case D, the magnetization curve E shows only two stages. This is because V1/V1' and V2/V2' walls move at the same speed (under the same driving force but in opposite directions), collide and annihilate simultaneously in case E. To highlight the roles of magnetostatic interactions to the magnetic property, it is worth comparing cases $\mathrm{A}$ and $\mathrm{E}$. 


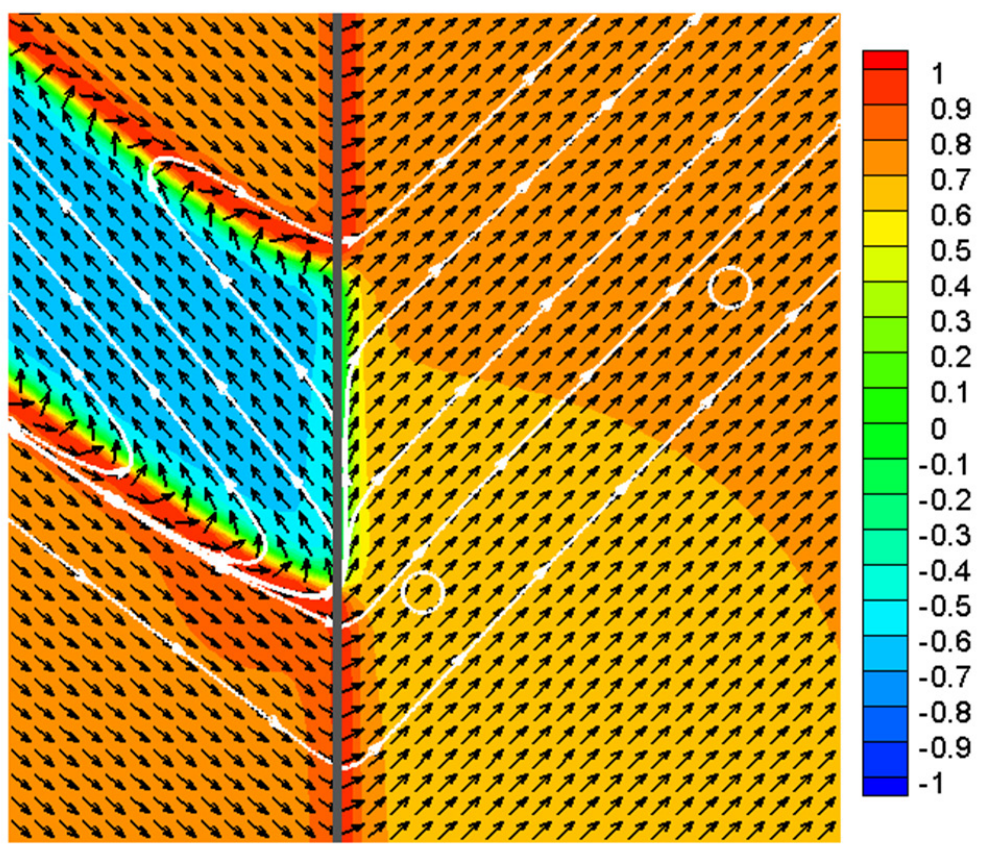

Figure 5.10 Close-up view of the highlighted rectangular region in the domain configuration $\mathrm{C} 2$ in Figure 5.7. Black arrows represent magnetization vector distribution, whose component in the twin normal direction is represented by color contours according to the color scale (same as in Figure 5.6). White stream lines illustrate the flux of magnetization vector field and serve as guide to eye to highlight small magnetization rotation and domain wall structures. The yellow patch in the orange background in the twin variant 1 manifests heterogeneous magnetization distribution due to the internal magnetic field. In particular, magnetization vector highlighted by the white circle in the orange patch orients $45^{\circ}$ while that in the yellow patch orients $48^{\circ}$ from the twin normal direction. The magnetization distribution in variant 2 reveals the detailed structures of two $180^{\circ}$ walls, across which magnetization vectors rotate towards external magnetic field direction: clockwise in the upper wall while anticlockwise in the lower wall.

From Figure 5.6, it can be easily understood that, if not considering the magnetostatic interactions, cases $\mathrm{A}$ and $\mathrm{E}$ would give the same magnetic responses. Comparing the magnetization curves $\mathrm{A}$ and $\mathrm{E}$, two differences can be identified: case $\mathrm{E}$ shows shallower slope in early stage but greater height in later stage, which can be explained by the 
differences in the magnetic domain structures of $\mathrm{A} 1$ and $\mathrm{E} 1$, and $\mathrm{A} 2$ and $\mathrm{E} 2$ respectively. An increase in magnetostatic interaction due to the domain wall splitting in E1 requires higher external magnetic field to move magnetic domain walls than in A1 where the continuity of $180^{\circ}$ domain walls maintains. This explains the lower slope of curve E. The internal magnetic field generated by the magnetic charges at twin boundaries in E2 causes additional magnetic domain rotation toward external magnetic field, contributing to higher sample magnetization.

It would be desirable to compare the simulated magnetization curves with experimental measurements. However, such a comparison is hindered by the fact that the simulations and experiments are performed on very different length scales of hierarchical structures in FePt samples: the simulation considers elementary domain processes occurring in representative polytwin structures, which are the common structural elements of FePt crystals; while experiments usually measure macroscopically averaged magnetization of the whole sample, which consists of many polytwin structures that are crystallographically equivalent but of different orientations, thus responding differently to an external magnetic field. Local measurement of magnetization response in one polytwin structure would be required. On the other hand, simulation of magnetization curves of a whole sample consisting of many polytwin structures of different orientations would be desired, which, however, is beyond the reach of computer simulation given the currently available computing power even with parallel supercomputers as used in this work. Nevertheless, direct comparison of simulated domain evolution processes with experimental observations are possible, because domain patterns and their responses to external fields have been experimentally observed, ${ }^{163,170,171}$ which show good agreement between simulation and experiments, such as coordinated motion, pinning, and bending of the domain walls and domain configuration composed of single-domain and multidomain twin variants, as shown in Figure 5.7.

It is worth noting that understanding of the elementary domain processes and magnetic responses of representative polytwin structures is a necessary step towards a 
better understanding of magnetization curves and structure-property relationships in FePt samples. It will help interpret and predict the magnetic properties of macroscopic samples, which are the results of collective contributions from many elementary processes. This study presents our simulation studies in this direction, and further investigation is under way as our ongoing research. The simulation results presented show detailed domain evolutions and highly anisotropic local magnetic properties, and reveal underlying domain mechanisms for magnetization processes in a polytwin structure. Furthermore, we quantitatively analyze the important contributions of magnetostatic interaction and domain wall motions in polytwin structures, as also indicated by experimental investigations, ${ }^{170,}{ }^{171}$ which is not captured by theoretical studies by only considering magnetization rotation mechanism. ${ }^{168}$ Due to the large anisotropy of FePt, magnetic domain wall motion plays the dominant role in the magnetization processes, while magnetization rotation is significant only at late-stage magnetization process when external magnetic field is very high. 


\section{Chapter 6. Conclusion and discussion}

To conclude this thesis, we first summarize the DIFA model, the simulation results and the insights learnt from the simulation as well as theoretical analyses. The potential and perspective of the DIFA model are discussed.

\subsection{Summary about the DIFA model on colloidal systems}

On the basis of Wang and Millett's founder work ${ }^{64-66}$ of diffuse interface field approach (DIFA) on modeling colloidal systems, this thesis has further developed the model on multi-phase-liquid colloid system by introducing the Gibbs-Duhem type formula of Laplace pressure, which makes the DIFA model capable of quantitatively studying capillarity related phenomena in complex multi-phase fluid colloid system. By employing the scheme of diffuse interface, the particles of arbitrary shapes can be captured without explicitly tracking the surfaces/interfaces. Owing to the templated field variables customized for each particle, the DIFA model is able to describe complex heterogeneities with charge/dipole distribution on the surfaces and/or inside the bodies of particles. Other heterogeneities such as nonuniform surface affinity, core-shell structured particles with varying dielectric/magnetic susceptibility can also be dealt with conveniently by field variables.

In detail, the Gibbs-Duhem type formula consistent with the Cahn-Hilliard theory is introduced to treat the thermodynamic pressure in multi-phase fluid system, conforming to Young-Laplace equation, whose direct application would be difficult for complex evolving colloidal morphology. The model is thus able to study a large group of capillarity phenomena in multi-phase fluid colloids or gels, such as lateral capillary interaction due to external forces or surface morphological fluctuations. The steric force is treated by introducing a "halo" field for each particle which is uniformly thicker than the particle surface. The short range steric force is thus formulated by using the intrinsic 
gradient effects of diffuse interfaces, and it is pairwise additive in nature. Such halo-field scheme may also work for modeling core-shell particles. By introducing a virtual "halo" field, the surface tension can be more accurately treated which makes the contact angle conforming to Young's equation better.

The diffuse interface method has advantage that the interfaces are not required to be explicitly tracked. The complex contacts between particles are thus conveniently treated without calculate the real-time gap distance between contact surfaces. The spectral method is frequently applied in DIFA, which attributes to the smooth transition of field variables at diffuse interfaces such that the error of discrete Fourier Transforms is largely reduced. The spectral method and parallel scheme makes DIFA highly efficient in numerical implementation. The well scalability makes the algorithm suitable to be implemented in distributed memory parallel environment, which is the most costeffective parallel system and widely applied (c.f. http://www.top500.org) .

\subsection{Insights from modeling and computer simulations}

The advantage of numerical simulation is that it provides detailed mechanism which can be difficult or very expensive to directly observe in experiments. Some main insights from the modeling work in this thesis are listed as:

1) At liquid-liquid interfaces, despite of particle shape, in $2 \mathrm{D}$ at equilibrium isolated particles do not perturb the geometry of liquid interface unless external force is applied on them. Nevertheless, in 3D it is possible that the undulated interface by contact angle constraints would cause lateral capillary interaction between nonspherical particles.

2) For Pickering emulsions, positive Laplace pressure leads to spheroidized emulsion droplets, while negative Laplace pressure leads to shrinkage and collapse of the emulsion droplets; irregular shaped colloidal shell may stay under zero Laplace pressure due to zero effective surface tension. These conclusions are shown to be independent of particle shapes. 
3) When particles are jamming at liquid interfaces without non-capillary interparticle forces, the actual Laplace pressure is not determined by the apparent curvature of the contour shape of particles any more, or alternatively, the effective interfacial tension is altered. The actual Laplace pressure can instead be detected by measuring the size of a coexisting free droplet, which offers an indirect approach to measure the in situ short-range interparticle force-separation relationship.

4) For pendular capillary bridges between close colloidal particles, there exists a positive pressure-liquid volume relationship, which produces a self-stabilization mechanism, in analogy to the communicating vessels effect, operating through diffusive equilibrium of two-phase liquid morphologies. Such mechanism is in contrast to the common coarsening processes, rendering desired microstructural stability and uniformity to the capillary bridges, which can be used to reinforce the colloidal microstructures with considerable adhesion strength.

5) Charge heterogeneity may generates many intriguing phenomena, such as apparent like-charge attraction, dynamic equilibrium of charged particle aggregation, and dependence of aggregate size on charge heterogeneity, the dominating factors lie in competition between net charge - net charge Colombic interaction, dipole-dipole interaction, and Brownian motion.

6) When dispersed dipolar colloids are aligned under external field, a constant and relatively weak external field $H_{0}$ may well align the particles into parallel fibers, while if the external field is applied delayed, the colloids may spontaneously form ring-structures due to dipole-dipole interaction. The ring structures, however, do not contribute to the performance improvement of the composites, and it requires significantly higher external field to break the rings than the constant value $H_{0}$.

In additition, extended studies on dipole-dipole interaction have been applied on ferroelectric and ferromagnetic single crystal or polycrystal systems. It has been concluded that: 
1) The short-range ordering of charged point defects in ferroelectric materials provides a restoring force to the domain recovery, which is the aging-domain stabilization mechanism. At microscopic level, charge compensation causes the shoulder type dipole $\left(\mathrm{Fe}_{\mathrm{Ti}}^{\prime}-\mathrm{V}_{\mathrm{O}}^{* \bullet}-\mathrm{Ti}_{\mathrm{Ti}}^{\prime}\right)^{\times}$to be stable. The "L" type dipole-dipole interaction is proposed to be a suitable candidate responsible for providing the internal electric field, which coincides with phase field simulation and some clues from EPR experiments.

2) Dipole-dipole interaction between magnetic domains in neighboring twin crystals generates "magnetic charges" at the twin boundaries, which produce an internal magnetic field. The competition between the internal and external magnetic fields determines magnetic domain structures and their evolution paths, explaining the different characteristics of the magnetization curves under applied magnetic fields along different directions

\subsection{Further Discussion}

DIFA has demonstrated powerful capability to model complex self-assembly processes of colloidal particles as a mesoscale modeling method. Its unique strength lies in studying particles with arbitrary shapes, and heterogeneous surface/bulk properties, such as charge/dipole distribution, surface affinity, and various core-shell structures of particles as well as multi-phase carrier fluids. In the current DIFA model, the hydrodynamic interaction between particles is not included, although such effect is not expected to take important roles in most problems studied in this thesis where particles are assumed to undergo only quasistatic motion in viscous liquids. Without solving fluid dynamics, under small Reynolds number assumption the Stokeslet type hydrodynamic interaction can be incorporated in a simplified manner by fully introducing the method of Stokesian dynamics, ${ }^{60,172}$ if necessary. For spherical particles, the Oseen tensor ${ }^{1,63,173}$ may further simplify the processing of hydrodynamic interaction. Within the framework of continuum mechanics, however, coupling the DIFA with fluid mechanics is yet very challenging, which is due to the highly complicate boundary conditions that might be 
computationally intractable for conventional computational fluid dynamics. From this viewpoint, Lattice Boltzmann Method (LBM) does offer a promising route to combine with DIFA. By using discrete fictitious fluid particles, LBM has much adaptability to the geometric boundaries and pioneering work has been reported on combining LBM with diffuse interface methods. ${ }^{51-54,174-176}$ Combination of LBM with DIFA has the potential to offer a solution scheme for colloid self-assembly processes involving moderate or large Reynolds number cases, which will open up new area for DIFA to study rheological behavior of colloid suspension.

The DIFA model is expected to have a wide application prospect. In three dimensional cases, particles with nonspherical shape at liquid-liquid interfaces lead to undulation of liquid interfaces due to the contact angle constraint at the three-phase contact line, which may produce lateral capillary interaction. Such an effect has been utilized as a new route for particle self-assembly directed by liquid interfaces. ${ }^{87,88}$ The heterogeneity effects studied in this thesis are merely the tip of the iceberg. Actually many topics, such as core-shell structures, dipolar colloids, charge heterogeneity may be studied further and deeper. The combination of particle shape anisotropy and property/charge heterogeneity is expected to generate more and more interesting selfassembly phenomena and it may also enrich self-assembled structures and tuning methods. In addition, particle packing and solid state sintering can be studied by DIFA. Pioneered work was also attributed to Wang. ${ }^{177}$ Computer modeling and simulations could not only reveal the detailed mechanism of physics that cannot or is too expensive to be obtained in experiments, but also explore and try some undeveloped controlling methods at will, and advance experimental investigations, as in general the former is much more cost effective than the latter. 


\section{References}

(1) Russel, W. B.; Saville, D. A.; Schowalter, W. R., Colloidal dispersions. Cambridge University Press: Cambridge, UK, 1989.

(2) van Blaaderen, A. Mrs Bulletin 2004, 29, 85-90.

(3) Whitesides, G. M.; Boncheva, M. Proceedings of the National Academy of Sciences of the United States of America 2002, 99, 4769-4774.

(4) Glotzer, S. C.; Solomon, M. J. Nature Materials 2007, 6, 557-562.

(5) Li, F.; Josephson, D. P.; Stein, A. Angewandte Chemie-International Edition 2011, 50, 360-388.

(6) Galisteo-Lopez, J. F.; Ibisate, M.; Sapienza, R.; Froufe-Perez, L. S.; Blanco, A.; Lopez, C. Advanced Materials 2011, 23, 30-69.

(7 ) Dinsmore, A. D.; Hsu, M. F.; Nikolaides, M. G.; Marquez, M.; Bausch, A. R.; Weitz, D. A. Science 2002, 298, 1006-1009.

(8) Shevchenko, E. V.; Talapin, D. V.; Kotov, N. A.; O'Brien, S.; Murray, C. B. Nature 2006, 439, 55-59.

(9) Leunissen, M. E.; Christova, C. G.; Hynninen, A. P.; Royall, C. P.; Campbell, A. I.; Imhof, A.; Dijkstra, M.; van Roij, R.; van Blaaderen, A. Nature 2005, 437, 235-240.

(10) Caruso, F. Advanced Materials 2001, 13, 11-22.

(11) Romano, F.; Sciortino, F. Nature Materials 2011, 10, 171-173.

(12) Yethiraj, A.; van Blaaderen, A. Nature 2003, 421, 513-517.

(13 ) Stebe, K. J.; Lewandowski, E.; Ghosh, M. Science 2009, 325, 159-160.

(14) Zheludev, N. I. Science 2010, 328, 582-583.

(15) Yablonovitch, E. Physical Review Letters 1987, 58, 2059-2062.

(16 ) Braun, P. V.; Rinne, S. A.; Garcia-Santamaria, F. Advanced Materials 2006, 18, 2665-2678.

(17) Clegg, P. S. Journal of Physics-Condensed Matter 2008, 20.

(18) Goyal, A.; Hall, C. K.; Velev, O. D. Soft Matter 2010, 6, 480-484. 
(19) Kim, E.; Stratford, K.; Adhikari, R.; Cates, M. E. Langmuir 2008, 24, 6549-6556.

(20) Lee, M. N.; Mohraz, A. Advanced Materials 2010, 22, 4836-+.

(21) Stratford, K.; Adhikari, R.; Pagonabarraga, I.; Desplat, J. C.; Cates, M. E. Science 2005, 309, 2198-2201.

(22) Herzig, E. M.; White, K. A.; Schofield, A. B.; Poon, W. C. K.; Clegg, P. S. Nature Materials 2007, 6, 966-971.

(23) Clegg, P. S.; Herzig, E. M.; Schofield, A. B.; Egelhaaf, S. U.; Horozov, T. S.; Binks, B. P.; Cates, M. E.; Poon, W. C. K. Langmuir 2007, 23, 5984-5994.

(24) Subramaniam, A. B.; Abkarian, M.; Stone, H. A. Nature Materials 2005, 4, 553556.

(25) Anderson, V. J.; Lekkerkerker, H. N. W. Nature 2002, 416, 811-815.

(26) Pusey, P. N.; Vanmegen, W. Nature 1986, 320, 340-342.

(27) Gasser, U. Journal of Physics-Condensed Matter 2009, 21.

(28) Auer, S.; Frenkel, D. Nature 2001, 409, 1020-1023.

(29) Israelachvili, J., Intermolecular and surface forces. Academic press limited: New York, NY 1991.

(30) Liang, Y.; Hilal, N.; Langston, P.; Starov, V. Advances in Colloid and Interface Science 2007, 134-35, 151-166.

(31) Verwey, E. J. W.; Overbeek, J. T. G., Theory of the stability of lyophobic colloids. Elsevier Publishing Company, Inc.: Amsterdam, 1948.

(32) Derjaguin, B. V.; Landau, L. D. Acta Physicochim URSS 1941, 14, 633-662.

(33) Fritz, G.; Schadler, V.; Willenbacher, N.; Wagner, N. J. Langmuir 2002, 18, 6381-6390.

(34) Xu, H.; Kirkwood, J.; Lask, M.; Fuller, G. Langmuir 2010, 26, 3160-3164.

(35) Kralchevsky, P. A.; Nagayama, K. Langmuir 1994, 10, 23-36.

(36) Kralchevsky, P. A.; Nagayama, K. Advances in Colloid and Interface Science 2000, 85, 145-192.

(37) Cheng, T.-L.; Wang, Y. U. Langmuir 2012, 2696-2703.

(38) Heady, R. B.; Cahn, J. W. METALLURGICAL TRANSACTIONS 1970, 1. 
(39) Chen, W.; Tan, S. S.; Ng, T. K.; Ford, W. T.; Tong, P. Physical Review Letters 2005, 95 .

(40 ) Chen, W.; Tan, S. S.; Zhou, Y.; Ng, T. K.; Ford, W. T.; Tong, P. Physical Review E 2009, 79.

(41) Drelich, J.; Wang, Y. U. Advances in Colloid and Interface Science 2011, 165, 91-101.

(42) Wang, Y. U. class notes of Computational materials science and engineering 2010.

(43) Squires, T. M.; Brenner, M. P. Physical Review Letters 2000, 85, 4976-4979.

(44) Chen, J. C.; Kim, A. S. Advances in Colloid and Interface Science 2004, 112, 159-173.

(45) Zhang, Z. L.; Glotzer, S. C. Nano Letters 2004, 4, 1407-1413.

(46) Hore, M. J. A.; Laradji, M. Journal of Chemical Physics 2007, 126.

(47) Boek, E. S.; Coveney, P. V.; Lekkerkerker, H. N. W.; vanderSchoot, P. Physical Review E 1997, 55, 3124-3133.

(48) Yan, L. T.; Popp, N.; Ghosh, S. K.; Boker, A. Acs Nano 2010, 4, 913-920.

(49) Davis, J. R.; Panagiotopoulos, A. Z. Journal of Chemical Physics 2008, 129, 7.

(50 ) Capone, B.; Pierleoni, C.; Hansen, J. P.; Krakoviack, V. Journal of Physical Chemistry B 2009, 113, 3629-3638.

(51) Aidun, C. K.; Clausen, J. R. Annual Review of Fluid Mechanics 2010, 42, 439472.

(52) Stratford, K.; Adhikari, R.; Pagonabarraga, I.; Desplat, J. C. Journal of Statistical Physics 2005, 121, 163-178.

(53) Briant, A. J.; Yeomans, J. M. Physical Review E 2004, 69.

(54) Ladd, A. J. C.; Verberg, R. Journal of Statistical Physics 2001, 104, 1191-1251.

(55) Nguyen, N. Q.; Ladd, A. J. C. Physical Review E 2002, 66.

(56) Kay, D.; Welford, R. Siam Journal on Scientific Computing 2007, 29, 2241-2257.

(57 ) Yue, P. T.; Feng, J. J.; Liu, C.; Shen, J. Journal of Fluid Mechanics 2004, 515, 293-317. 
(58) Lowengrub, J.; Truskinovsky, L. Proceedings of the Royal Society of London Series a-Mathematical Physical and Engineering Sciences 1998, 454, 2617-2654.

(59) Anderson, D. M.; McFadden, G. B.; Wheeler, A. A. Annual Review of Fluid Mechanics 1998, 30, 139-165.

(60 ) Happel, J.; Brenner, H., Low Reynolds number hydrodynamics with special applications to particulate media. Noordhoff International Publishing: Leyden, The Netherlands, 1973.

(61 ) Landau, L. D.; Lifshitz, E. M., Fluid Mechanics. Reed Educational and Professional Publishing Ltd: Oxford, 1997.

(62 ) Vafeas, P.; Dassios, G. Journal of Mathematical Physics 2006, 47.

(63) Swanson, E.; Teller, D. C.; Haen, C. D. Journal of Chemical Physics 1978, 68, 5097-5102.

(64) Wang, Y. U. Acta Materialia 2007, 55, 3835-3844.

(65) Millett, P. C.; Wang, Y. U. Journal of Colloid and Interface Science 2011, 6.

(66 ) Millett, P. C.; Wang, Y. U. Acta Materialia 2009, 57, 3101-3109.

(67) Cheng, T.-L.; Wang, Y. U. to be published.

(68) Zhang, Z. L.; Horsch, M. A.; Lamm, M. H.; Glotzer, S. C. Nano Letters 2003, 3, 1341-1346.

(69) Quere, D. Annual Review of Materials Research 2008, 38, 71-99.

(70 ) Drelich, J.; Miller, J. D.; Good, R. J. Journal of Colloid and Interface Science 1996, 179, 37-50.

(71) Jiang, S.; Chen, Q.; Tripathy, M.; Luijten, E.; Schweizer, K. S.; Granick, S. Advanced Materials 2010, 22, 1060-1071.

(72) Madivala, B.; Fransaer, J.; Vermant, J. Langmuir 2009, 25, 2718-2728.

(73) Ye, X. C.; Collins, J. E.; Kang, Y. J.; Chen, J.; Chen, D. T. N.; Yodh, A. G.; Murray, C. B. Proceedings of the National Academy of Sciences of the United States of America 2010, 107, 22430-22435.

(74) Chen, L. Q. Annual Review of Materials Research 2002, 32, 113-140.

(75 ) Boettinger, W. J.; Warren, J. A.; Beckermann, C.; Karma, A. Annual Review of Materials Research 2002, 32, 163-194. 
(76) Defay, R.; Prigogine, I.; Bellemans, A.; Everett, D. H., Surface tension and adsorption. Longmans, Green: London, 1967.

(77) Khan, S. J.; Pierce, F.; Sorensen, C. M.; Chakrabarti, A. Langmuir 2009, 25, 13861-13868.

(78 ) Butt, H. J.; Kappl, M. Advances in Colloid and Interface Science 2009, 146, 4860.

(79) Ball, R. C.; Melrose, J. R. Physica a-Statistical Mechanics and Its Applications 1997, 247, 444-472.

(80 ) Cahn, J. W.; Hilliard, J. E. Journal of Chemical Physics 1958, 28.

(81) Zhu, J. Z.; Chen, L. Q.; Shen, J.; Tikare, V. Physical Review E 1999, 60, 35643572.

(82) Chen, L. Q.; Shen, J. Computer Physics Communications 1998, 108, 147-158.

(83) Xu, H.; Lask, M.; Kirkwood, J.; Fuller, G. Langmuir 2007, 23, 4837-4841.

(84) Cheng, T. L.; Huang, Y. X. Y.; Rogers, C. M.; Jin, Y. M. M. Journal of applied physics 2010, 107.

(85) Jin, Y. M. M. Acta Materialia 2009, 57, 2488-2495.

(86 ) Jin, Y. M. M.; Wang, Y. U.; Kazaryan, A.; Wang, Y. Z.; Laughlin, D. E.; Khachaturyan, A. G. Journal of applied physics 2002, 92, 6172-6181.

(87) Danov, K. D.; Kralchevsky, P. A. Advances in Colloid and Interface Science 2010, 154, 91-103.

(88) Lewandowski, E. P.; Bernate, J. A.; Searson, P. C.; Stebe, K. J. Langmuir 2008, 24, 9302-9307.

(89) Irvine, W. T. M.; Vitelli, V.; Chaikin, P. M. Nature 2010, 468, 947-951.

(90) ) Datta, S. S.; Shum, H. C.; Weitz, D. A. Langmuir 2010, 26, 18612-18616.

(91 ) Waugh, A.; Grant, A., Anatomy and physiology in health and illness. 9th ed.; Elsevier Science Ltd.: London, UK, 2007.

(92 ) Monteux, C.; Kirkwood, J.; Xu, H.; Jung, E.; Fuller, G. G. Physical Chemistry Chemical Physics 2007, 9, 6344-6350.

(93) Binks, B. P. Current Opinion in Colloid \& Interface Science 2002, 7, 21-41. 
(94) Willett, C. D.; Adams, M. J.; Johnson, S. A.; Seville, J. P. K. Langmuir 2000, 16, 9396-9405.

(95) Fisher, R. A. The Journal of agricultural science 1926, 16, 12.

(96) Hornbaker, D. J.; Albert, R.; Albert, I.; Barabasi, A. L.; Schiffer, P. Nature 1997, $387,765-765$.

(97) Hanna, G.; Barnes, W. J. P. Journal of Experimental Biology 1991, 155, 103-125.

(98) Koos, E.; Willenbacher, N. Science 2011, 331, 897-900.

(99) Qian, J.; Gao, H. J. Acta Biomaterialia 2006, 2, 51-58.

(100) Vogel, M. J.; Steen, P. H. Proceedings of the National Academy of Sciences of the United States of America 2010, 107, 3377-3381.

(101 ) Gogelein, C.; Brinkmann, M.; Schroter, M.; Herminghaus, S. Langmuir 2010, 26, 17184-17189.

(102) Mitarai, N.; Nori, F. Advances in Physics 2006, 55, 1-45.

(103 ) Iveson, S. M.; Litster, J. D.; Hapgood, K.; Ennis, B. J. Powder Technology 2001, 117, 3-39.

(104) Voorhees, P. W. Journal of Statistical Physics 1985, 38, 231-252.

(105) Lifshitz, I. M.; Slyozov, V. V. Journal of Physics and Chemistry of Solids 1961, $19,35-50$.

(106) Jang, J. Y.; Schatz, G. C.; Ratner, M. A. Journal of Chemical Physics 2002, 116, 3875-3886.

(107) Maeda, N.; Israelachvili, J. N.; Kohonen, M. M. Proceedings of the National Academy of Sciences of the United States of America 2003, 100, 803-808.

(108) Restagno, F.; Bocquet, L.; Biben, T. Physical Review Letters 2000, 84, 2433-2436.

(109) Page, A. J.; Sear, R. P. Physical Review Letters 2006, 97.

(110) Evans, R. Journal of Physics-Condensed Matter 1990, 2, 8989-9007.

(111) Evans, R.; Marconi, U. M. B. Journal of Chemical Physics 1987, 86, 7138-7148.

(112 ) Fisher, L. R.; Israelachvili, J. N. Nature 1979, 277, 548-549.

(113) Fisher, L. R.; Israelachvili, J. N. Journal of Colloid and Interface Science 1981, 80, 528-541. 
(114) Fratzl, P.; Lebowitz, J. L.; Penrose, O.; Amar, J. Physical Review B 1991, 44, 4794-4811.

(115) Bykov, T. V.; Zeng, X. C. Journal of Chemical Physics 2002, 117, 1851-1868.

(116) Kuni, F. M.; Shchekin, A. K.; Rusanov, A. I.; Widom, B. Advances in Colloid and Interface Science 1996, 65, 71-124.

(117 ) Wang, J. F.; Wang, Z. G.; Yang, Y. L. Journal of Chemical Physics 2004, 121, 1105-1113.

(118) It has to be noted that at regions beside a curved fluid interface, the order parameters have slight deviation from exact 1 or 0 , caused by the chemical potential shift due to the Laplace pressure effect.

(119) Beysens, D.; Esteve, D. Physical Review Letters 1985, 54, 2123-2126.

(120) Allen, S. M.; Cahn, J. W. Acta Metallurgica 1979, 27, 1085-1095.

(121) Thijssen, J. H. J.; Clegg, P. S. Soft Matter 2010, 6, 1182-1190.

(122) Sanz, E.; White, K. A.; Clegg, P. S.; Cates, M. E. Physical Review Letters 2009, 103.

(123) Blanco, A.; Chomski, E.; Grabtchak, S.; Ibisate, M.; John, S.; Leonard, S. W.; Lopez, C.; Meseguer, F.; Miguez, H.; Mondia, J. P.; Ozin, G. A.; Toader, O.; van Driel, H. M. Nature 2000, 405, 437-440.

(124) Dodds, S.; Carvalho, M. D.; Kumar, S. Physics of Fluids 2009, 21.

(125) Eggers, J. Reviews of Modern Physics 1997, 69, 865-929.

(126) Erb, R. M.; Son, H. S.; Samanta, B.; Rotello, V. M.; Yellen, B. B. Nature 2009, 457, 999-1002.

(127) Paunov, V. N.; Kralchevsky, P. A.; Denkov, N. D.; Nagayama, K. Journal of Colloid and Interface Science 1993, 157, 100-112.

(128) Islam, A. M.; Chowdhry, B. Z.; Snowden, M. J. Advances in Colloid and Interface Science 1995, 62, 109-136.

(129) Weidenfeller, B.; Anhalt, M.; Riehemann, W. Journal of Magnetism and Magnetic Materials 2008, 320, E362-E365.

(130) Wang, Y. U. Applied Physics Letters 2010, 96. 
(131) Jona, F.; Shirane, G., Ferroelectric crystals. Pergamon Press: Oxford, New York, 1962.

(132) Wang, Y. U. Journal of Materials Science 2009, 44, 5225-5234.

(133 ) Rao, W. F.; Cheng, T. L.; Wang, Y. U. Applied Physics Letters 2010, 96, 3.

(134) Ren, X. B. Nature Materials 2004, 3, 91-94.

(135) Mestric, H.; Eichel, R. A.; Kloss, T.; Dinse, K. P.; Laubach, S.; Schmidt, P. C.; Schonau, K. A.; Knapp, M.; Ehrenberg, H. Physical Review B 2005, 71, 10.

(136 ) Sun, D. Z.; Ren, X. B.; Otsuka, K. Applied Physics Letters 2005, 87.

(137) Zhang, L. X.; Erdem, E.; Ren, X. B.; Eichel, R. A. Applied Physics Letters 2008, $93,3$.

(138 ) Amin, A.; Haun, M. J.; Badger, B.; McKinstry, H.; Cross, L. E. Ferroelectrics 1985, 65, 107-130130.

(139) Ren, X. B.; Otsuka, K. Physical Review Letters 2000, 85, 1016-1019.

(140) Jonker, G. H. Journal of the American Ceramic Society 1972, 55, 57.

(141 ) Lambeck, P. V.; Jonker, G. H. Journal of Physics and Chemistry of Solids 1986, 47, 453-461.

(142) Arlt, G.; Neumann, H. Ferroelectrics 1988, 87, 109-120.

(143 ) Warren, W. L.; Pike, G. E.; Vanheusden, K.; Dimos, D.; Tuttle, B. A.; Robertson, J. Journal of applied physics 1996, 79, 9250-9257.

(144) Kroger, F. A., The chemistry of imperfect crystals. North-Holland Publishing Company: Amsterdam, 1964.

(145) Eichel, R. A. Journal of the American Ceramic Society 2008, 91, 691-701.

(146 ) Eichel, R. A.; Erhart, P.; Traskelin, P.; Albe, K.; Kungl, H.; Hoffmann, M. J. Physical Review Letters 2008, 100, 4.

(147 ) Pontin, R. G.; Slade, E. F.; Ingram, D. J. E. Journal of Physics C 1969, 2, 1146.

(148) Laguta, V. V.; Glinchuk, M. D.; Bykov, I. P.; Maksimenko, Y. L.; Rosa, J.; Jastrabik, L. Physical Review B 1996, 54, 12353.

(149) Keeble, D. J.; Loyo-Menoyo, M.; Booq, Z. I. Y.; Garipov, R. R.; Eremkin, V. V.; Smotrakov, V. Physical Review B 2009, 80. 
(150) Lewis, O.; Wessel, G. Physical Review B (Condensed Matter and Materials Physics) 1976, 13, 2742.

(151) Erhart, P.; Eichel, R. A.; Traskelin, P.; Albe, K. Physical Review B 2007, 76.

(152 ) Tealdi, C.; Malavasi, L.; Fisher, C. A. J.; Islam, M. S. Journal of Physical Chemistry B 2006, 110, 5395-5402.

(153) Siegel, E.; Muller, K. A. Physical Review B (Condensed Matter and Materials Physics) 1979, 20, 3587.

(154 ) Mestric, H.; Eichel, R. A.; Dinse, K. P.; Ozarowski, A.; van Tol, J.; Brunel, L. C.; Kungl, H.; Hoffmann, M. J.; Schonau, K. A.; Knapp, M.; Fuess, H. Physical Review $B$ 2006, 73, 10.

(155 ) Batllo, F.; Duverger, E.; Jules, J. C.; Niepce, J. C.; Jannot, B.; Maglione, M. Ferroelectrics 1990, 109, 113-118.

(156) Raymond, M. V.; Smyth, D. M. Journal of Physics and Chemistry of Solids 1996, 57, 1507-1511.

(157 ) Blennow, P.; Hagen, A.; Hansen, K. K.; Wallenberg, L. R.; Mogensen, M. Solid State Ionics 2008, 179, 2047-2058.

(158) Poykko, S.; Chadi, D. J. Physical Review Letters 1999, 83, 1231-1234.

(159) Ivanov, O. A.; Solina, L. V.; Demshina, V. A.; Magat, L. M. Fiz. Met. Metalloved 1973, 35, 92.

(160 ) Liu, J. P.; Luo, C. P.; Liu, Y.; Sellmyer, D. J. Applied Physics Letters 1998, 72, 483.

(161 ) Suzuki, T.; Muraoka, H.; Nakamura, Y.; Ouchi, K. IEEE Trans. Magn. 2003, 39, 691.

(162 ) Khachaturyan, A. G., Theory of structural transformations in solids. Wiley: New York, NY, 1983.

(163) Vlasova, N. I.; Shur, Y. S. Phys. Met. Metall 1987, 63, 69.

(164) Zhang, B.; Soffa, W. A. Phys. Status Solidi A 1992, 131, 707.

(165) Zhang, B. Scripta Met. Mater. 1994, 30, 683.

(166 ) Klemmer, T.; Hoydick, D.; Okumura, H.; Zhang, B.; Soffa, W. A. Scripta Metallurgica et Materialia 1995, 33, 13. 
(167) Tanaka, Y.; Kimura, N.; Hono, K.; Yasuda, K.; Sakurai, T. Journal of Magnetism and Magnetic Materials 1997, 170, 289.

(168) Vlasova, N. I.; Kandaurova, G. S.; Shchegoleva, N. N. Journal of Magnetism and Magnetic Materials 2000, 222, 138.

(169 ) Kazaryan, A.; Wang, Y.; Jin, Y. M. M.; Wang, Y. U.; Khachaturyan, A. G.; Wang, L. S.; Laughlin, D. E. Journal of applied physics 2002, 92, 7408-7414.

(170) Armstrong, J. N.; Sullivan, M. R.; Le Romancer, M.; Chernenko, V. A.; Chopra, H. D. Journal of applied physics 2008, 103.

(171 ) Lai, Y. W.; Scheerbaum, N.; Hinz, D.; Gutfleisch, O.; Schafer, R.; Schultz, L.; McCord, J. Applied Physics Letters 2007, 90.

(172 ) Kutteh, R. Physical Review E 2004, 69.

(173) Koga, T.; Kawasaki, K. Physical Review A 1991, 44, R817-R820.

(174) Kim, E.; Stratford, K.; Cates, M. E. Langmuir 2010, 26, 7928-7936.

(175 ) Jansen, F.; Harting, J. Physical Review E 2011, 83.

(176 ) Pooley, C. M.; Kusumaatmaja, H.; Yeomans, J. M. Physical Review E 2008, 78.

(177) Wang, Y. U. Acta Materialia 2006, 54, 953-961. 


\section{Work published or to be published during PhD study:}

Peer reviewed journal papers:

- T.L. Cheng, Y.U. Wang, Self-Assembly and Aggregation of Charge Heterogeneous Colloidal Particles: A Computational Study, (manuscript in preparation)

- T.L. Cheng, Y.U. Wang, Shape-Anisotropic Particles at Curved Fluid Interfaces and Role of Laplace Pressure: A Computational Study, (to be submitted)

- T.L. Cheng, I. Kuianov, Y.U. Wang, Charge Compensation and Aging Mechanisms in Iron-Doped Ferroelectric Titanate Perovskites, (manuscript in preparation)

- T.L. Cheng, Y.U. Wang, Spontaneous Formation of Stable Capillary Bridges for Firming Compact Colloidal Assemblies during Liquid Phase Separation: A Computational Study, Langmuir (2012) DOI: 10.1021/la2044152

- J.E. Zhou, T.L. Cheng, Y.U. Wang, Correlated Nucleation and SelfAccommodating Kinetic Pathway of Ferroelectric Phase Transformation, Journal of Applied Physics (2012) DOI: 10.1063/1.3677993

- T.L. Cheng, F.D. Ma, J.E. Zhou, G. Jennings, Y. Ren, Y.M. Jin, Y.U. Wang, In-Situ Three Dimensional Reciprocal Space Mapping of Diffuse Scattering Intensity Distribution and Data Analysis for Precursor Phenomenon in Shape Memory Alloy, JOM 64(2012) 167-173

- W.F. Rao, K.W. Xiao, T.L. Cheng, J.E. Zhou, Y.U. Wang, Control of Domain Configurations and Sizes in Crystallographically Engineered Ferroelectric Single Crystals: Phase Field Modeling, Applied Physics Letters 97(2010) 162901 (Featured as Cover Image)

- W.F. Rao, T.L. Cheng, Y.U. Wang, Aging-Stabilization of Ferroelectric Domains and Internal Electric Field due to Short-Range Ordering of Charged Point Defects: Phase Field Modeling, Applied Physics Letters 96 (2010) 122903

- T.L. Cheng, Y.Y. Huang, C.M. Rogers, Y.M. Jin, Micromagnetic Modeling of Magnetization Processes in FePt Polytwin Crystals, Journal of Applied Physics 107 (2010) 113920. 
Conference presentations:

As speaker:

- T.L. Cheng, Y.U. Wang, Modeling the Self-Assembly of Arbitrary-Shaped FerroColloidal Particles in Bulk Liquid and at Fluid Interface, TMS Annual Meeting, San Diego, CA, Feb. 27-Mar.03, 2011

- T.L. Cheng, Y.U. Wang, Computer-Aided Design, Processing and Characterization of Polymer-matrix Magnetic Composites, TMS Annual Meeting, San Diego, CA, Feb. 27-Mar.03, 2011

- T.L. Cheng, Y.U. Wang, Charged Point Defect Configurations, Domain Stabilization, and Ferroelectric Aging, TMS Annual Meeting, San Diego, CA, Feb. 27-Mar.03, 2011

- T.L. Cheng, Y.M. Jin, Y. Huang, Magnetization Processes in FePt Polytwin Crystals, MS\&T Conference, Pittsburgh, PA, Oct. 26-29, 2009.

- T.L. Cheng, W.F. Rao, Y.U. Wang, Aging-Stabilization of Ferroelectric Domains due to Charged Point Defects Short Range Ordering, MS\&T Conference, Pittsburgh, PA, Oct. 26-29, 2009.

As coauthor:

- J.E. Zhou, T.L. Cheng, Y.U. Wang, Effects of Filler Microstructures on Effective Properties of Magnetic Composites: Phase Field Modeling and Simulation, TMS Annual Meeting, San Diego, CA, Feb. 27-Mar.03, 2011

- J.E. Zhou, T.L. Cheng, Y.U. Wang, Correlated Nucleation and SelfAccommodating Kinetic Pathway of Ferroelectric Phase Transformation, TMS Annual Meeting, San Diego, CA, Feb. 27-Mar.03, 2011

- J.E. Zhou, T.L. Cheng, K.W. Xiao, W.F. Rao, Y.U. Wang, Control of Domain Configurations and Sizes in Crystallographically Engineered Ferroelectric Single Crystals: Phase Field Modeling, TMS Annual Meeting, San Diego, CA, Feb. 27Mar.03, 2011

- Y.U.Wang, X. Zhao, T.L. Cheng, G. Jennings, Y. Ren, Three-Dimensional Reciprocal Space Mapping of Martensitic Transformations in Bulk Single Crystals by In Situ High-Energy Synchrotron X-ray Diffraction, TMS Annual Meeting, San Diego, CA, Feb. 27-Mar.03, 2011

- Y.M. Jin, T.L. Cheng, Y. Huang, Domain Mechanisms for Magnetic Field Induced Deformation, MS\&T Conference, Pittsburgh, PA, Oct. 26-29, 2009. 